\title{
COMPELIING CHOICES: TENSIONS IN THE PRACTICE AND EXPERIENCE OF PRENATAL SCREENING IN NEW ZEALAND
}




\section{Compelling choices:}

Tensions in the practice and experience of prenatal screening in New Zealand

by

Sarah Ellen Donovan

A thesis

Submitted to the Victoria University of Wellington in fulfilment of the requirements for the degree of Doctor of Philosophy in Sociology

Victoria University of Wellington 


\section{Abstract}

Recently there has been a shift in health policy in New Zealand, as internationally, away from prenatal screening for fetal abnormalities targeting specific 'high risk' groups of pregnant women, towards the implementation of population-based screening programmes. As a result, for the majority of pregnant women in Western countries, prenatal screening is now undertaken as part of the standard 'package' of maternity care. This thesis explores the constitution of prenatal screening as a now taken-for-granted aspect of contemporary pregnancy care. It considers tensions between popular and medical understandings of the value and purpose of this practice, in particular examining the contradictory nature of discourses which, on one hand, construct acceptance of prenatal screening as an empowering 'choice' available to pregnant women, and on the other hand, as an institutionally-endorsed, morallyappropriate practice of risk management. Recent developments within the New Zealand context are presented as a 'case study' of the representational politics of prenatal screening within contemporary public health policy.

The question of informed consent for prenatal screening is a key focus within the research. It examines the extent to which the scope of women's choices and the degree of consent possible are materially shaped and constrained within the context of current clinical practice, and the broader climate of contemporary pregnancy care. Empirically, the research investigates key themes in women's experience of prenatal screening decisions within this climate, and explores the private 'moral work' undertaken by pregnant women as they navigate the contradictory imperatives which circulate in mainstream screening discourse. The findings of the research suggest a 
need for clinicians and policy makers to recognise prenatal screening as an ethically complex 'special case', qualitatively distinct from other projects of population-based health screening. It is argued that ethical practice in prenatal screening requires an approach which acknowledges the discursive and material factors which problematise liberal conceptions of prenatal screening as an authentic, deliberative 'choice' available to pregnant women. 


\section{Acknowledgements}

First and foremost my sincere thanks go to the twenty participants who took part in the interviews for this thesis, women who shared their experiences with a degree of candour and personal generosity that continues to remind me what a privileged task I had in undertaking this research. I hope you will feel that I have presented your stories accurately, usefully, and with due sensitivity.

To my supervisors Mike Lloyd and Rhonda Shaw, thank you both for consistent patience and encouragement, and for conveying a sense of implicit faith in my ability to 'get there eventually'. I am extremely grateful for this approach. It is difficult to know how to offer adequate thanks for the roles you have variously played in helping me reach this milestone.

To my brother Richard, for boundless good humour, generosity and inner calm in the face of turbulent times. I am very much in your debt.

I am also grateful to the following fabulous friends for supporting and inspiring me along the way: Amanda Berkahn, Jaqui Knowles, Kim Paton, Helen Cain, Jayanthi Ramasinghe, Jeffrey Lind, Sandy Gibbs \& Chris Williams, Chris Goldsbury, Jane Duthie, Chamsey El-Ojeili, Josie Di Bella, Di O'Connell, Grant Ellen, Sheryl Hann, Tim Corballis, Ingrid Horrocks, Emma Hislop, Sarah \& Alex Clark, Alison Hogg and Charlotte Yates.

Thank you also to the friendly bunch of postgraduate students on the ninth floor of the Murphy building, especially Kathy Glasgow for shared thesis humour, inspiration, and tolerance of my 'filing system' in a small office.

The Victoria University crèche were always wonderfully helpful and caring during my daughter's time there, and I feel lucky to have had access to such a high-quality childcare during my studies. I am also greatly appreciative for the receipt of a Victoria University $\mathrm{PhD}$ Scholarship, which provided in valuable financial assistance.

My sincere thanks also go to the New Zealand Women's Studies Association for the receipt of a Rosemary Seymour Award, a most encouraging endorsement of my topic which also provided useful financial support.

In addition to my supervisors, a number of other academic staff within the School of Social and Cultural Studies also offered ongoing encouragement during the course of the PhD, especially Allison Kirkman, Jenny Neale and Kevin Dew. I am also most grateful to the administrative staff of SACS, especially Monica Lichti, for helping me out so many times, always with great warmth. I will miss the friendly 'vibe' of the School office. 
To my daughter Rosa, my companion on this journey since toddlerhood; thank you for waiting so patiently for "mum's big black book" to finally be finished.

And to my partner Harriette for putting up with it all, for so many delicious meals, and for "getting the jokes".

This thesis is dedicated, with love, to my parents, Jo and Peter Donovan. 


\section{Table of contents}

Abstract $\quad$ ii-iii

Acknowledgments iv-v

Chapter One 1

Positioning prenatal screening as a matter of Sociological interest

Aims of the research 1

Defining prenatal screening 5

Positioning the research $\quad 8$

$\begin{array}{ll}\text { Positioning the researcher } & 10\end{array}$

Background to the research 12

$\begin{array}{ll}\text { Theoretical approach } & 15\end{array}$

$\begin{array}{ll}\text { Methodological approach } & 19\end{array}$

$\begin{array}{ll}\text { The case for thematic analysis } & 21\end{array}$

$\begin{array}{ll}\text { The study participants } & 23\end{array}$

Ethics approval and cultural considerations 28

Overview of chapters 29

Chapter Two 32

From 'high risk' to mainstream: the routinisation of prenatal ultrasound screening for fetal abnormality

Introduction $\quad 32$

What is ultrasound? $\quad 33$

Overview of the development of obstetric ultrasound 35

$\begin{array}{ll}\text { The safety of prenatal ultrasound } & 37\end{array}$

Clinical reservations relating to prenatal ultrasound screening 39

Issues around practitioners' role: technical competence and patient

counselling 42

The trajectory of routinisation $\quad 44$

Recent developments in prenatal imaging: nuchal translucency scanning 47

Routinising ultrasound screening: implications for pregnant women 52

The phenomenon of incorrect diagnoses 52

Routine ultrasound screening and maternal anxiety around pregnancy

as inherently 'risky' 55

Is 'informed consent' for prenatal ultrasound currently a reality? 
Themes in the experience of prenatal screening

$\begin{array}{ll}\text { Purpose of the empirically-based chapters } & 62\end{array}$

$\begin{array}{ll}\text { Scope of participants' experience } & 64\end{array}$

General attitudes towards the experience of prenatal screening 65

Views on termination of pregnancy 66

$\begin{array}{ll}\text { Attitudes towards disability } & 67\end{array}$

Key themes in participants' experience $\quad 68$

$\begin{array}{ll}\text { Reassurance and control } & 69\end{array}$

Scans as a source of enjoyment: "it's just nice to see your baby" 72

"Treating a heavy thing as a light thing": attitudes towards the representation of prenatal screening 73

The experience of risk $\quad 77$

Insufficiency of information $\quad 80$

"The right not to know" $\quad 84$

$\begin{array}{ll}\text { Acceptance and surrender } & 87\end{array}$

$\begin{array}{ll}\text { Disembodiment and provisionality } & 88\end{array}$

\section{Chapter Four}

Women's screening decisions as 'moral work'

'Trading-off' unwelcome aspects of experience 97

Embodied knowledge as a moral resource $\quad 99$

$\begin{array}{ll}\text { Time as a moral resource } & 102\end{array}$

$\begin{array}{ll}\text { Metaphor as a moral resource } & 107\end{array}$

Reinscribing clinical facts as social reality 111

$\begin{array}{ll}\text { Experiential projects of pregnancy } & 115\end{array}$

Refusing prenatal screening 117

How are 'refusals' medically and socially judged? 121

$\begin{array}{ll}\text { Conclusions on the analysis of interview data } & 124\end{array}$

\section{Chapter Five}

Choice or compulsion? Ethical issues in prenatal screening: the question of informed consent.

$\begin{array}{lr}\text { Prenatal screening as an emergent 'public health' concern } & 129\end{array}$

Risk discourse in public health: ethical implications $\quad 135$

$\begin{array}{ll}\text { An alternative model } & 138\end{array}$

$\begin{array}{ll}\text { Informed consent } & 140\end{array}$

$\begin{array}{ll}\text { The problem with informed consent for prenatal screening } & 142\end{array}$

Risk and consent 143

Significance of the practitioner-patient relationship for informed consent $\quad 149$

Ruby's story: a case study of the problematic of informed consent for prenatal screening. 
The New Zealand experience: the representational politics of prenatal screening as a contemporary public health concern

Prenatal screening in New Zealand: a brief history 163

Current practice in New Zealand 166

Historic limitations on screening as a 'choice' for New Zealand women 169

A mood for change: prenatal screening within recent public health discourse

in New Zealand

Future directions: a national Down syndrome screening programme by any other name?

Chapter Seven

Fetal imaging as 'spectacular medicine': exploring the boundaries of contemporary medical uses of prenatal ultrasound.

Prenatal ultrasound: channelling 'desire'? 196

Recent developments in fetal imaging 198

Merging fact and fetish: the evolution of fetal-maternal 'bonding' discourse 207

Theorising clinicians' extra-scientific uses of fetal imaging 213

$\begin{array}{ll}\text { Prenatal ultrasound as 'spectacular medicine' } & 213\end{array}$

'Show and tell': sonography as a vicarious pleasure? 215

\section{Chapter Eight}

Conclusion: Revisiting consent: prenatal screening as an ethical 'special case'.

What do pregnant women seek in their engagement with prenatal screening?

Facilitating informed consent: some pragmatic suggestions

Recognising the significance of time and place as contexts of decision-making

Practitioners' role as representational agents of prenatal screening: the need for professional training and support

Addressing the normative significance of ultrasound

\section{Appendices}

$1 \quad$ Glossary of clinical terms 251

2 List of abbreviations 255

3 Information sheet for participants 256

4 Interview Questions 258

$5 \quad$ Feature article on the research 260

6 Consent form for participants 261

$7 \quad$ List of approved indications for obstetric ultrasound 262

$8 \quad$ Nuchal translucency scan image 263

$9 \quad$ Statement on ultrasound, New Zealand College of Midwives 264 
10 Media sample 'Rise in Down syndrome babies blamed on lack of screening'

11 Media release 'Report on antenatal Down syndrome screening released by the National Screening Unit'

$12 \quad$ Media release 'Down syndrome antenatal screening under review' 273

13 Media release 'DPA disputes antenatal screening claims' 275

14 National Screening Unit, 'Background to the quality improvements' 276

15 Media release 'Screening on the way for Down syndrome babies' 278

16 Terms of reference, Toi te Taiao/Bioethics Council of New Zealand. 281

Bibliography 


\section{Chapter One}

\section{Positioning prenatal screening as an issue of sociological interest - Research Approach}

\section{Aims of the research}

This thesis is a sociological exploration of prenatal screening (and in particular, prenatal ultrasound scanning) as a normal and normative aspect of contemporary pregnancy care. The purpose of the research is first, to explore prenatal screening in terms of an 'everyday', yet largely culturally invisible, experience; and secondly, to locate this experience within the broader social context of contemporary pregnancy care.

Why is prenatal screening of sociological interest? Prenatal screening is a complex medical and cultural phenomenon, beset with contradictions. For pregnant women, as those on the 'receiving end' of these technologies, popular and medical expectations around screening as a 'norm' of pregnancy care generate an array of contradictory imperatives. For example, within New Zealand, contemporary primary health care models promote a view of pregnancy and birth as normal life events, and midwifery practice endorses a non-interventionist approach as an ideal for the care of uncomplicated pregnancies. However, as theorists of public health and risk culture have suggested, routine screening for fetal abnormality may cast a shadow of uncertainty and a sense of personal 'riskiness' over an otherwise unproblematic life experience. As I will argue in this thesis, the constitution of pregnancy as at once 'normal' and requiring routine technomedical scrutiny, problematises 'commonsense' understandings of the purpose and value of practices such as prenatal screening. 
The research is both empirical and theoretical in scope. The examination of prenatal screening as both a clinical practice and as a subjective experience requires a broad scope of enquiry. For this reason the thesis approaches the phenomenon from a number of different angles. This multiple approach comprises qualitative analysis of interview data with descriptive content (technical and historical), critical examination of relevant texts, and broader theoretical discussion. The empirical data is examined in the light of available social scientific literature and also in relation to recent clinical/public health literature. I scrutinize this latter material as significant among the various cultural 'texts' ${ }^{1}$ which contribute to contemporary understandings of prenatal screening within the practice of maternity care.

Little is known about New Zealand women's experiences or attitudes towards this now 'everyday' aspect of pregnancy care. ${ }^{2}$ Therefore a key aim of this thesis is to address this gap in local sociological knowledge, as well as to locate this work in relation to existing international research. A further aim of this empirical approach is to contribute to a more nuanced understanding of the landscape of pregnancy as a contemporary health-oriented experience. In particular, the discussion of interview data explores prenatal screening in terms of a contemporary everyday 'moral moment' in the lives of pregnant women. Two key questions were first, what themes emerge in contemporary experiential accounts of prenatal screening? Secondly, how do pregnant women themselves navigate this ethically complex terrain? In addressing these questions, the thesis aims to contribute to and extend existing inquiry into the moral worlds of lay people in their engagement with contemporary practices of health

\footnotetext{
${ }^{1}$ This approach follows Rapp's treatment of medical literatures on prenatal screening as 'cultural texts' (1999).

2 Oakley's 1997 MA thesis in health sciences is the only known qualitatively-oriented research undertaken on this topic in New Zealand.
} 
care. More broadly, the research situates these experiences within the context of the various 'truths' of prenatal screening as an everyday aspect of contemporary pregnancy care.

The thesis title (Compelling Choices) reflects a key concern of my research, that is, do pregnant women experience prenatal screening as a choice or as a compulsion within the context of the current 'package' of prenatal care? As I will suggest, this question has specific resonance both within the New Zealand setting and overseas, where innovations in screening technologies and shifts in public health policy have seen a move away from targeting 'high risk' pregnancies (for example, screening only older mothers) towards a routine, population-based approach to prenatal screening.

In particular this research considers tensions between the theory and the practice of prenatal screening, and specifically, tensions between contemporary public health discourses which represent such screening as a matter of individual 'choice', and the reality of day to day screening practice in the clinical setting, where prenatal screening is typically presented to women as a standard, everyday aspect of pregnancy care. As a public health concern, screening for fetal abnormalities is uniquely different from other projects. It can be seen as emblematic of the concerns of what has been referred to as 'the new public health' (Petersen \& Lupton, 1996), that is an emphasis on public education and health promotion, alongside the ethos of individual responsibility and decision-making around personal health, as distinct from a prior emphasis on the prevention of infectious disease (Dew \& Kirkman, 2002).

However, as I will suggest, in its aims prenatal screening is distinct from other forms of population-based screening, and the positioning of this practice in this way raises a number of unaddressed issues which problematise liberal assumptions of choice and 
consent. ${ }^{3}$ These tensions, and their implications for individual pregnant women, are the focus of the interview-based component of the research.

The past two decades have seen an increasing popular and social scientific fascination with technologies and practices often referred to under the rubric of 'the new genetics'. This has been especially true in the area of reproductive health, where, in particular, developments in technologies of assisted fertility (otherwise known as 'assisted reproductive technologies', or ARTS) can now be seen to constitute a key focus in clinical research, and certainly to dominate media coverage of reproductive health issues generally. As social scientific commentators on emerging genetic technologies note, ${ }^{4}$ this emphasis on genetic 'solutions' in healthcare has accelerated a contemporary shift away from more traditional therapeutic models towards a promotion of a preventive approach, a trend encapsulated in the flourishing of 'preventive medicine' as a new sub-discipline of biomedicine. This perception of genetics in health as an 'emerging' or 'new' focus appears to have led to a waning of critical interest in already established genetic-related technologies, in particular, prenatal ultrasound screening. While highlighting a number of problematic (and arguably unresolved) aspects of the use of prenatal ultrasound, social scientific interest in this practice, having peaked in the late 1980s and early 1990s, now appears to have dwindled significantly. While the ethnographic work produced during this time made a convincing case for the need to revisit the ethics of this now widespread practice, it seems clear that prenatal screening, in the form of its current widespread use, is no longer a sociological 'hot topic'.

\footnotetext{
${ }^{3}$ The emergence of a trend to constitute prenatal screening in terms of a contemporary 'public health' good will be discussed further in chapter five.

See for example, Petersen \& Bunton, 2002, Kerr \& Cunningham-Burley, 2000, Lupton, 1995,

Lippman 1991, 1992.
} 


\section{Defining prenatal screening}

The term 'prenatal screening' is broadly used to refer to a number of methods used to detect fetal abnormalities prior to birth, including ultrasound scans, amniocentesis, ${ }^{5}$ maternal blood tests, and more recently, pre-implantation genetic diagnosis (PGD). Of these screening methods, prenatal ultrasound is by far the most widely-used throughout the world, and is commonly employed to identify signs of a number of specific chromosomal disorders, in particular Down syndrome, cystic fibrosis and spina bifida. The use of ultrasound screening for Down syndrome is a particular focus of my research, as the detection of this condition continues to be a key focus in prenatal screening programmes, both internationally and within New Zealand (see in particular chapters two and six). As a method of screening for fetal abnormality, prenatal ultrasound is functionally linked to the selective termination of pregnancy. This is primarily due to the fact that no therapeutic remedies are currently available to correct conditions such as Down syndrome prior to birth. Also referred to as Trisomy 21 , Down syndrome is one of the most frequently-occurring non-lethal chromosomal disorders in humans. ${ }^{6}$ Clinical studies indicate that a majority of pregnant women will choose to terminate a pregnancy affected by this condition. No data is kept in New Zealand on reasons for termination of pregnancy, however European research notes a $92 \%$ rate of termination following diagnosis of Down syndrome (Mansfield et al, 1999). Outside the clinical domain, purely pathological evaluations of Down syndrome are subject to debates around the value and rights of the disabled, and the alleged 'eugenic agenda' of genetics-based screening practices more generally. This research is concerned with investigating current practices of prenatal screening and

\footnotetext{
${ }^{5}$ See appendix 1 for a glossary of clinical terms used throughout the thesis. Please note that the wide use of the term 'screening', which is often used interchangeably with 'diagnosis', should be seen to reflect a failure to recognize a significant clinical distinction.

${ }^{6}$ See chapter two for specific discussion of Down syndrome.
} 
does not engage with debates around the moral permissibility of selective termination of pregnancy for fetal abnormality, other than to flag such perspectives as among the various discourses circulating in popular or 'mainstream' understandings of prenatal screening.

During pregnancy, women are normally offered several forms of screening, (including blood and urine tests, and sometimes vaginal swabs) to measure relevant variables such as iron levels, signs of STDs, and immunity to Rubella (Medforth et al, 2006). Such tests are often referred to as antenatal screening tests, however it should be noted that within both popular and clinical literature relating to pregnancy, the terms 'antenatal' and 'prenatal' are sometimes used interchangeably. This can cause confusion, not least because pregnancy screening involves two sets of evaluations screening for maternal wellbeing as well as for fetal wellbeing. For the purposes of this research the term 'prenatal screening' should therefore be recognized as referring specifically to the assessment of fetal wellbeing, and in particular, the evaluation of genetic normality of the fetus.

Within the United States, 'prenatal' has historically been the preferred term, and this has been increasingly adopted internationally. However it is worth noting that within a number of countries, including the United Kingdom and New Zealand, the term 'antenatal screening' continues to be used within clinical circles. ${ }^{7}$ Increasingly, the two terms are used interchangeably in these settings.

\footnotetext{
${ }^{7}$ See for example the recent New Zealand "Report on Antenatal Screening for Down syndrome" (Stone \& Austen, 2006)
} 
Within New Zealand, it is clear that there is considerable variation in the ways prenatal screening is both formally defined and popularly understood. This seems likely to be linked to the fact that historically the approach taken to screening in New Zealand has not been co-ordinated at a national level, but rather has involved screening being offered by maternity practitioners to individual women on an 'ad hoc' basis. ${ }^{8}$ With no official position on the practice having ever been taken by the Ministry of Health, prenatal screening in New Zealand appears to have evolved chiefly in line with the whim of interested clinicians, rather than subject to formalised (and arguably more consultative or 'democratic') processes of public policy development.

This has, I would suggest, contributed to a high degree of terminological inconsistency and variable representation of the practice within the popular media in New Zealand, as well as within the domains of public health and bioethics. The recent emergence of terms such as 'pre-birth testing' as a catch-all descriptor for all forms of pregnancy screening (both maternal and fetal, and including embryonic genetic testing) has added to this ambiguity, and suggests the scope of misunderstanding possible when clinical practices evolve in an uncoordinated manner) As this thesis will argue, both locally and overseas, a historic lack of clarity and consistency in the presentation of prenatal screening to the lay public seems likely to have contributed to a phenomenon of widespread misunderstanding about the clinical purpose and sequelae of prenatal screening.

\footnotetext{
${ }^{8}$ The specifics of screening practice in New Zealand are discussed in more detail in chapter six.
} 


\section{Positioning the research}

Existing research on prenatal screening needs to be recognized as having been produced within several different domains which are broadly epistemologically distinct. As might be expected, clinical research overwhelmingly assumes a functional view of disability, therefore generally positioning prenatal screening in terms of broader projects of risk management or the avoidance of disability as a negative health outcome. In line with this perspective, clinical literature on this topic is generally concerned with matters of technical efficacy and efficiency, with prenatal screening widely presumed to exist as a medical/public health good. ${ }^{9}$

In contrast, research on this topic produced outside the clinical domain, which has predominantly been from within the social sciences (and in particular, cultural anthropology) ${ }^{10}$ and allied fields such as nursing studies, has tended to take a more critical perspective. This is no doubt in part due to a focus on personal accounts within research undertaken in these disciplines which, as will be discussed later in the thesis, has often signalled an uneasy fit between the various clinical and experiential 'truths' of this practice. Clinical research on prenatal screening historically has not included qualitative research, and findings from social scientific studies typically are not included within literature reviews for medical research. As this thesis will argue, this apparent reluctance within contemporary obstetrics to acknowledge the shortcomings of current screening practices at the level of subjective experience (a fact now increasingly recognized within the social sciences), and to treat this as a

\footnotetext{
${ }^{9}$ However it is important to note the existence of a small body of clinical literature which takes a more critical perspective on the assumed benefits of current screening practice. See chapter two for further discussion.

${ }^{10}$ In particular the work of Raynor Rapp, Sarah Franklin, and more recently Janelle Taylor.
} 
matter of clinical ethics, ${ }^{11}$ suggests an ongoing need for social scientific critique and commentary in this area.

During the course of my research I became increasingly interested in the empirical work sociologists can contribute to ethical debates, and this intention came to broadly direct my research aims. In this respect, this research fits within an emerging body of sociological work which engages with questions around the ethics of contemporary healthcare practices. Such work emphasizes the importance of an empirical focus in analyses of bioethical processes, stressing the significance of ethnographic data.

Recent work in this area is increasingly characterised by attempts to trace the "moral work' undertaken by individuals involved in complex health decisions, and involves qualitative ethnographic research with both lay people (often patients/healthcare consumers), and health practitioners (see chapter five for further). Implicit in such research is an intention that such contributions enable pragmatic shifts in clinical practice leading to more positive experiences for individuals engaging with healthcare (Lopez, 2004). This orientation towards an applied approach to qualitative health research has increasingly seen the emergence of an inherently inter-disciplinary brand of research; often this has been undertaken by social scientists who themselves have a practical background in health and the biological sciences (for example, the work of Claire Williams, Sarah Franklin, Jackie Leach Scully, and Margarete Sandelowski). Such work has offered a useful resource in setting the scope, tone and methodological approach of my own research.

\footnotetext{
${ }^{11}$ By 'clinical ethics' I refer to a concern within the clinical setting for the practical application of ethical principles commonly associated with medical practice, such as the imperative for clinicians to do no harm (non-maleficence) and to follow protocols of informed consent.
} 
This notion of the humanizing potential of sociologists' role in contributing to bioethical debates appealed to my sense as a clinician ${ }^{12}$ that women's experience and attitudes towards screening practices may not be receiving sufficient ethical attention, and culminated in my desire to address this lack in my capacity as a sociologist with an 'insider' perspective. Participants interviewed for this thesis frequently expressed a wish that their involvement might have a practical value in drawing attention to what they clearly viewed as problematic aspects of prenatal screening. In the spirit of this sentiment, it is hoped that this exploratory contribution to local research might create a platform for further enquiry into lay experiences of health decisions in New Zealand. My own hope that the accounts of participants highlighted in this research make available a useful 'ethical resource' for the development of policy in this area reflects Lopez's contention that

"to the extent that ethnographers feel that they can add something to the discourse of bioethics, then they must also be committed to finding a place for their knowledge claims not only amongst their sociological peers but more broadly in the public sphere".

(Lopez, 2004:880).

\section{Positioning the researcher}

My interest in this issue first arose during my experience as a midwife working within the New Zealand hospital system. In this environment it occurred to me that the day to day practice of prenatal screening as an ostensibly 'neutral' element of pregnancy care did not adequately acknowledge the inherent moral complexity of this experience, or the extent to which such choices may, for some women, exist as a virtually 'inescapable' burden within this context (Donovan, 2006). Far from viewing prenatal

\footnotetext{
${ }^{12}$ See below, next section.
} 
screening as 'just another test', as it often appears to be understood within popular pregnancy discourse, pregnant women I encountered frequently expressed considerable ambivalence about this aspect of their pregnancy experience, which was often recalled as a rather anxious and uncertain time. This was particularly the case for first time mothers. In this context I tended to meet pregnant women long after such decisions had been made, however, I became curious about how pregnant women experienced the imperative to address the 'choices' available to them, and what this process might involve for them personally.

Both the individual experiences and the day to day practices of prenatal screening appeared to be in tension with the prevailing rhetoric of individual autonomy and informed consent now so roundly emphasised within health policy in New Zealand. I thus came to view the current representation and implementation of prenatal screening as an intriguing ethical 'blind spot' in the local landscape of contemporary healthcare.

Aware of the introduction of novel screening techniques in New Zealand at this time, including the nuchal translucency scan, ${ }^{13}$ I attempted to find out what sort of resources and support were available for pregnant women facing these decisions.

However, I was surprised to find very little in the way of pamphlets or other official information about screening available for public education. Moreover, there appeared to be no guidelines for the maternity practitioners relied upon to counsel and support women around this sensitive issue. An examination of New Zealand data on the topic revealed a handful of local clinical articles on 'best practice' in prenatal screening, but no specific investigation of the social or ethical dimensions of this practice, and

\footnotetext{
${ }^{13}$ See chapter two.
} 
certainly no qualitative research evaluating the needs and attitudes of pregnant women themselves. Similarly, virtually no New Zealand based social scientific research on this issue was available. With this apparent scarcity of local knowledge in mind, I began my interviews.

\section{Background to the research}

The social and ethical implications of prenatal screening first began to attract social scientific attention during the late nineteen eighties, a movement spearheaded by the ethnographic work of cultural anthropologist Raynor Rapp in the United States. Rapp's qualitative interview-based studies highlighted the individual and social impact of this practice, and in particular, the unacknowledged ethical dimension or 'moral work' associated with of this experience. In her analysis, society at large relied upon individual pregnant women to privately confront and absorb uncomfortable ethical dilemmas (chiefly, the ethics of selective termination) associated with these emerging technologies. She thus characterised pregnant women as "moral pioneers" (1987:101) within the context of high-tech innovations in practices of pregnancy care. Concurrently and in a similar vein, Barbara Katz Rothman's research on the experience of amniocentesis signalled the negative psychological consequences of prenatal screening. She first coined the notion of the 'tentative pregnancy' (1986) to describe the way the institutionalization of such practices may lead women to emotionally disconnect from their pregnancies, and to view them as inherently provisional. Both characterisations of the contemporary experience of pregnancy have been extremely influential in subsequent social scientific work in this area, although, as will be discussed later, it is notable that the findings of these and subsequent qualitative studies appear not to have made a substantive impact on current practice 'in the field'. Critical of medicine's apparent lack of interest in the 'down stream' 
impact of such technologies, and the assumption of women's unproblematic acceptance of them, Rapp's work signalled the need to situate women's pregnancy experiences, including their attitudes towards specific practices of pregnancy care, within the broader social context of their lives, accounting for structural variables such as ethnicity, socio-economic status and religious background (1998). Her work however focused less on the significance of cultural discourse in terms of a structural constraint on women's decision-making. This research seeks to develop this line of analysis. A further aim is to extend and 'flesh out' Rapp's insights around the private moral work undertaken by pregnant women facing prenatal screening decisions. Rapp's early research in this area was generated within the broader context of contemporary feminist critique of the medicalisation of childbirth in Western maternity care. At this time, the dominant 'medical model' approach to birth (and pregnancy care more generally) as a highly-managed event was increasingly the subject of feminist critique, an angle subsequently taken up more broadly within social scientific analyses of the socially constructed nature of medical knowledge and practices of health care.

This thesis fits broadly within a body of social scientific enquiry into contemporary practices of reproduction. Such research has often been concerned with the representational politics of these practices, and the various ways women engage with and make sense of their experiences in this domain. While not always focussing on prenatal screening per se, related work on reproductive technologies such as that of Franklin $(1991,1997)$ and Cussins/Thompson $(1998,2005)$ provided useful theoretical tools for unpicking the complexity of prenatal screening as a phenomenon of contemporary culture. Existing inter-disciplinary research on the practice of fetal 
ultrasound imaging has also been influential in establishing the theoretical scope of my enquiry into contemporary screening practices, including the work of Mitchell \& Georges (1997, 1998), Sandelowski (1994a, 1998), Taylor (1998, 2000, 2002) and Weir (1996, 1998a, 1998b). Focussing specifically on prenatal screening practices in the USA in the 1990s, Press and Browner's work $(1994,1995,1996,1999)$ has also contributed greatly to my understanding the trajectory between older and newer screening methods, as well as the evolution of popular attitudes towards these practices.

More recently, the question of prenatal screening has been revisited in the light of innovations in contemporary practice. In particular, Williams et al's 2005 ethnographic work builds on Rapp's view of pregnant women as 'moral pioneers' relative to the implementation of novel techniques of first-trimester pregnancy screening. This work draws fresh attention to the ethical dilemmas generated by practices of prenatal screening, and signals the persistence of complex tensions between 'mainstream' and individual experiences in this area. This has also been a concern of recent ethnographic research exploring lay accounts of health decisions, and in particular the work of Garcia et al (2008a, 2008b) and Scully et al (2004, 2006a, 2006b, 2007). In framing screening decisions in terms of the 'moral worlds' of individuals facing this experience, such work, in my view, offers more nuanced insights into such experiences than previous social scientific research in this area. This close examination of the personal moral frameworks which underlie screening decisions is also the intention of the interview-based component of this thesis. 


\section{Theoretical approach}

A social scientific investigation of prenatal screening as both a practice and an experience has sometimes felt like a fiendishly complex affair. This is partly due to the inherently inter-disciplinary nature of the topic, and the associated necessity to engage with texts from somewhat disparate domains, including clinical medicine, social science, public health and popular media. However, as Rapp argued, women's screening decisions are not made 'in a vacuum' and therefore I would argue that any meaningful investigation of this experience in women's lives requires an examination of the broader ideological context of mainstream understandings of this practice.

While a significant aim of the thesis is to explore prenatal screening through an experiential prism, the complexity and scope of the phenomenon is such that a comprehensive analysis requires a considerably broader understanding of the phenomenon. The approach taken is intended to situate the various and sometimes competing 'knowledges' of prenatal screening within the sites where such meanings are produced and reproduced - including medical literature, public health material, popular discourse, existing sociological research, and within the context of women's own experience, as a site which has largely been culturally 'hidden'. This follows Rapp's view that attempts to chart the 'social impact and cultural meaning' (1998:46) of a phenomenon as complex as prenatal screening necessarily requires a 'multi-sited' research approach. This is an approach which broadly attends to the argument for 'triangulation, 14 as a means of increasing the validity and completeness of research findings, which as Begley (1996) suggests, depends as much on the combination of multiple data sources as it does on a mixed-method approach per se (1996).

\footnotetext{
${ }^{14}$ Bryman defines triangulation as "The use of more than one method or source of data in the study of a social phenomenon so that findings may be cross-checked" (2008:700).
} 
The inclusion of relevant technical and historical information within the thesis also reflects an attempt to take a sufficiently nuanced approach to the complexity of this topic. This information is provided to help establish a meaningful context for participant's subjective accounts. A glossary of medical/technical terms used in the thesis is included in the appendix for the benefit of readers who may not be unfamiliar with clinical knowledge in this area. ${ }^{15}$

In considering the extent to which prenatal screening is a socially constituted norm, rather than a self-evidently 'normal' practice of pregnancy care, this thesis follows a broadly social constructionist approach. The essence of social constructionist thought is the view that truth claims should not be seen as self-evidently or objectively true, but rather as products of socially-negotiated 'facts' or forms of knowledge. As Nettleton (1995:18) notes, a key presumption of social constructionism is that medicine should be recognized as a "political rather than a technically neutral enterprise". According to this perspective, the biomedical discourse around prenatal screening needs to be understood as among a range of existing 'stories', which circulate to lesser or greater degrees in what might be called mainstream or ‘commonsense' discourse. In examining prevalent medical and cultural discourses around pregnancy and prenatal care, as well as the day to day, material practices of prenatal screening in the clinical setting, this thesis attends to a core concern of social constructionist research which is to unpick the ways in which meaning is "constituted in and through discourse and social practices" (Lupton, 2003:12).

As discussion of the interview data will show, mainstream or 'commonsense' discourse around prenatal screening as a necessary, unproblematic aspect of

\footnotetext{
${ }^{15}$ See appendix 1. See also appendix 2 for a list of abbreviations used in the thesis.
} 
pregnancy care cannot be seen to speak the whole 'truth'; other less visible discourses have considerable significance for women's prenatal screening decisions. As Gavey notes (1997:52), "the dominant discourses appear "natural", denying their own partiality and gaining their authority by appealing to common sense. These discourses, which support and perpetuate existing power relations, tend to constitute the subjectivity of most people most of the time". In essence, this thesis is concerned with scrutinizing assumptions about "what most people most of the time" think about prenatal screening.

Social constructionism has been the dominant approach within the Sociology of Health and Illness, ${ }^{16}$ a sub-discipline of Sociology which emerged in the 1950s (Nettleton, 1995, Lupton 2003, Dew and Kirkman, 2002). This is also true of much related interdisciplinary work, which has often combined approaches from anthropology, cultural studies, feminist theory and nursing studies within this broad perspective. A key concern of this approach is exploring the significance of the social context of health experiences and practices. Other significant questions underpinning this perspective as it has been applied to issues of healthcare relate to the production and maintenance of medical knowledge as 'authoritative', the representational politics of various 'truth claims' relating to health, and explorations of lay understandings of health and illness. This research explores these questions through the prism of contemporary practices of prenatal screening.

Feminist research has been at the forefront of social constructionist commentary on the power relations underpinning scientific knowledge and practices. While this thesis

\footnotetext{
${ }^{16}$ A field of enquiry sometimes also referred to as 'Medical Sociology', particularly within the United States.
} 
does not draw explicitly on specific feminist approaches, the research shares much with feminist poststructuralist approaches to theorizing experience and identity. This includes an understanding of knowledge as socially produced and unstable, and an emphasis on the significance of discourse in shaping both collective and individual accounts of the world (Potter and Wetherell, 1987, Gavey, 1997). Furthermore, the research should be recognized as broadly feminist in the sense that it reflects my own views as a researcher that research focusing on women should address questions of inequality and power. This attends to a concern in feminist research with the ways medical knowledge and practices have tended to disregard the significance of gender for health outcomes, access to health care, and, most relevant to this research, the experience of receiving care (Lupton, 2003, Nettleton, 1995). Work with this orientation is implicitly concerned with the possibility of social change, and, as Gavey (1997:53) suggests, with the intention of "disrupting and displacing dominant (oppressive) knowledges". In line with this perspective on the purpose of social research, the empirical findings of this thesis are presented as a potential 'ethical resource', and the conclusion of the thesis has a pragmatic orientation which reflects the wish expressed by a number of participants that their involvement in the study make a useful contribution to clinical practice.

A good deal of feminist work in the area of women's health has historically assumed the overwhelming significance of 'medicalisation' in delimiting women's reproductive autonomy, the case sometimes being made (or more often implied) that women are, by and large, coerced and exploited in their engagement with reproductive technologies. It is not within the scope of this research to specifically reprise such arguments. As will be discussed later in the thesis, there is much 
evidence that pregnant women wish to have access to technologies such as prenatal screening, and it is not the intention of this research to suggest that this positive view reflects a generalized 'brainwashing' of pregnant women, or to argue that some women are 'more medicalised' than others. However, it is my position that within the context of a culture of routine screening, such acceptance does nonetheless warrant a critical eye, and cannot necessarily be seen to reflect a 'choice', that is, in the sense of a fully informed and/or whole-hearted decision. As Zechmeister (2001:397) suggests, "women's attitudes towards reproductive technology have to be treated cautiously as their attitudes exist within a social context, within existing power relations, social obligations, constraints, and traditions". From my perspective as both a social researcher and a midwife, I would argue that the degree to which contemporary prenatal care broadly reflects a 'medical model' approach needs to be recognized as a profoundly significant aspect of the ideological backdrop of women's engagement with prenatal screening and practices and expectations around maternity care more generally.

\section{Methodological approach}

The use of qualitative interviews is well recognized within the social sciences as a useful approach for the study of 'lived experience'. This is a concept from phenomenological research, an approach which asserts that it is possible to access shared 'truths' of human experience though subjective accounts (Byrne, 2001). As Kvale (1996) suggests, interviews are also a suitable method for enquiry into novel research areas, or where the subject matter is particularly complex. The purpose of the interviews in this study was to elicit in-depth subjective accounts of prenatal screening, and to explore participants' personal evaluations of the experience; in other 
words, to see how participants themselves 'made sense' of their screening encounters and how they accounted for their decisions. The aim of this research was to identify general experiential themes, rather than to claim homogeneity of experience. While the argument is often made by those conducting qualitative research that subjective accounts should be treated as 'authoritative' in their own right, participants' accounts are not presented within this research as conveying some essential or unified 'truth' about prenatal screening. As Farquhar (1996) argues, the 'essence' of reproductive technologies cannot be adequately captured by subjective accounts alone. Rather, these accounts are included to provide a local, contextualized counter-point to mainstream discourse.

The interviews were conducted in late 2005 and early 2006, at a place convenient to participants (in most cases, at their homes due to the presence of small children). Prior to interviews being conducted, participants were provided with an information sheet explaining the aims of the research. ${ }^{17}$ The interviews usually lasted between $50-80$ minutes, and were guided by a series of open-ended questions. ${ }^{18}$ These questions were used to broadly prompt participants in describing and evaluating their encounters with prenatal screening during the course of their pregnancy care. The use of semistructured, open ended questions rather than a strict schedule of questions was suitable for this research for a number of reasons. Firstly, this approach allowed participants to recall events on their own terms, which seemed appropriate to the highly personal nature of the experiences being recalled. In addition, this open ended approach was useful in creating a 'moral space' within which individual women felt

\footnotetext{
${ }_{18}^{17}$ See appendix 3.

${ }^{18}$ See appendix 4.
} 
comfortable accounting for their decisions. ${ }^{19}$ This commitment to a partially 'participant-directed' approach to data gathering also influenced the choice of thematic analysis as a suitable method of data analysis within the study. In coding and discussing data in terms of themes of concern, my approach borrowed from recent research exploring the ethical deliberations of lay people. ${ }^{20}$ As within this recent work, my research sought to pay particular attention to issues which participants themselves had identified as personally ethically problematic or complex. Given that the majority of participants expressed varying degrees of dissatisfaction with their screening experiences, the organization and analysis of data along these lines seemed appropriate. Several 'case studies ${ }^{21}$ are included within the thesis. Stake (2005) suggests that case studies offer a useful 'extension of experience' in discussion of qualitative data, and these have been included to add depth and provide useful background information to participants' comments.

\section{The case for thematic analysis}

The last several decades have seen increasing social scientific interest in research seeking to foreground 'lived experience', that is, where the primary data sought is individual subjective accounts of particular phenomena. In the analysis and organization of this data, such research has often laid claim to a wide and varied range of methodologies, from 'discourse analysis', to 'interview-based ethnography', ${ }^{22}$ 'phenomenology' and 'grounded theory'. On close inspection, such research can often be seen to share a common approach or method in the management of the data,

\footnotetext{
${ }^{19}$ See chapter five for a discussion of the significance of the notion of 'moral space' as a requirement 20 individual ethical decision-making.

${ }^{20}$ See especially Scully et al, 2004. For other examples see Smelzer, 1994, and Potts et al, 2003. More broadly the organization and analysis of data in terms of 'emergent themes' is methodologically

characteristic of the 'grounded theory' approach; see for example the work of Charmaz.

In the sense of extended discussions of particular experiential accounts.

See for example Draper, 2002.
} 
having been coded and analysed in terms of themes arising from the data itself. Nonetheless, in spite of the widespread use of this approach in contemporary qualitative research, researchers rarely classify their method in terms of 'thematic analysis'. This point is made by Boyatzis (1998) and also Braun and Clarke (2006:77) who argue that thematic analysis is a "poorly demarcated and rarely acknowledged, yet widely used qualitative analytic method". Certainly there is minimal specific literature available on the mechanics of thematic analysis as a method of qualitative analysis. This may be because of a perception of this method as somehow methodologically inferior to other qualitative approaches, or perhaps as too 'generic'. As Sandelowski (2000a:335) has suggested, those conducting qualitative research have sometimes felt the need to 'rebrand' more generic approaches to the management of qualitative data, and to engage in what she refers to as "methodological acrobatics" in the interests of establishing their work as sufficiently analytically complex (and therefore methodologically 'valid'). However, given the aims of the interviews within this research to offer an exploratory 'snapshot' of experience within the New Zealand context, a generic or broad-based qualitative approach such as thematic analysis seemed an ideal approach. Sandelowski argues that generic qualitative methods are well-suited to pragmatically-oriented research on health-related phenomena, suggesting that such approaches are

...especially amenable to obtaining straight and largely unadorned (i.e. minimally theorized or otherwise transformed or spun) answers to questions of special relevance to practitioners and policy-makers. Examples of such questions include: What are the concerns of people about an event? What are 
people's responses (e.g. thoughts, feelings, attitudes) toward an event? What reasons do people have for using or not using a service or procedure?

(Sandelowski, 2000a:337)

Boyatzis argues that thematic analysis is an ideal starting point for qualitative enquiry into elusive and complex phenomena, suggesting that especially when approaching multidisciplinary phenomena, this method is "probably the only way to begin thoughtful inquiry" (1998:viii). As he suggests, this approach is also valuable for its communicative potential, offering a highly accessible style of analysis important in research seeking to communicate phenomena to a wider audience. Thematic analysis fits well within research oriented to a social constructionist perspective, as, similar to discourse analysis, it can be used to draw links between patterns of individual experience and broader cultural discourses or 'meta-themes'. As Aronsen (1994) suggests the development of an interconnected 'storyline' between individual accounts and themes within the broader existing literature is useful in communicating the intention of the research to the reader. As with much research undertaken within a social constructionist framework (and particularly within the area of health), the accessibility of thematic analysis as a qualitative approach is also a good fit with the notion that such research should ultimately (at least to some extent) be 'geared toward change' (Tierney,1995).

\section{The Study participants}

Twenty participants were recruited for the study, the sole criterion for inclusion being that participants had received maternity care in New Zealand during a pregnancy within the last twenty years. Given the sensitive nature of the research topic and the need to ensure confidentiality of involvement, participant recruitment occurred on a 
self-selected basis. The majority of participants responded to a feature item about my proposed research in a national newspaper, ${ }^{23}$ with the remainder of the sample obtained through 'snowballing', having been made aware of the research by family or friends (Marshall, 1998). This approach to recruitment reflects the difficulty of accessing participants for studies of this nature, particularly within the local context where a somewhat fragmented approach to prenatal screening poses practical obstacles to advertising and recruitment through official avenues. The broad inclusion criterion similarly recognises this limitation, as well as the potentially restrictive number of willing participants given New Zealand's small population. As will be discussed further in chapter four, while no claim is made as to the representativeness of the study sample, the diversity of views expressed in participants' accounts do suggest a variety of motivations for taking part in the study; the sample can therefore be seen to stand as a 'snapshot' of a range of screening experiences.

In addition to taking part in the interview, participants were asked to provide a small amount of personal information to allow for a basic demographic profile of the research data; this included details of age and ethnicity, general occupational area, number of pregnancies and numbers of live children. To preserve anonymity, each participant was ascribed a pseudonym. ${ }^{24}$ Silverman (1993) suggests that the inclusion of a small amount of quantitative data adds depth to a qualitative study. Participants' accounts revealed a very broad range of experiences, and the following data is included as it may assist the reader to both contextualise and 'flesh out' participants accounts as discussed in chapters three and four. Participant details were broken down

\footnotetext{
${ }^{23}$ See appendix 5 .

${ }^{24}$ In the further interests of protecting participants' identity, the demographic data collected is not included in the appendix of the thesis. This is chiefly in recognition of the limited pool of potential participants within the New.Zealand context, and the small sample size of the study itself.
} 
as follows; at the time of interview, respondents ranged in age between 31 and 50 years of age. With respect to ethnicity, seventeen participants identified themselves as either Pakeha or New Zealand European, two as Maori, and one as a Pacific Islander. Participants were also invited to indicate their general occupational area on the consent form for the study. Of the twenty participants, five women described their primary occupation as "mother". The remaining participants indicated that they were currently in paid employment, with occupations in the areas of education, healthcare, manual labour and corporate fields represented within the sample. A high overall rate of tertiary-level education was noted.

Many pregnancies do not progress to the birth of a live infant. Futhermore, prenatal screening is functionally linked to the termination of pregnancies. As available literature suggests, the decision to terminate a pregnancy (whether wanted or 'unwanted') is for some women, a difficult and sometimes traumatic experience. I would suggest that where experiential accounts of pregnancy are the subject of research, it is necessary to acknowledge participants' experience of fetal loss, ${ }^{25}$ either through spontaneous miscarriage or termination of pregnancy, as part of the experiential scope of such research. For this reason, participants were asked to indicate both the number of pregnancies they had undergone as well as number of currently living children. This data showed that the twenty women collectively had forty nine children alive at the time of interview. Within the sample, twelve incidences of fetal loss were indicated, two of which were recorded specifically as medical terminations for fetal abnormalities detected through prenatal screening, in

\footnotetext{
${ }^{25}$ That is, fetal demise prior to birth
} 
one case for Down syndrome, and in another for a lethal genetic abnormality. ${ }^{26}$ One participant had lost a baby shortly after birth, due to a lethal abnormality detected during prenatal screening, a pregnancy she had chosen not to terminate, although aware of the likely outcome.

Participants were asked to indicate whether they had undergone prenatal screening during their pregnancies, and it was explained that such screening included ultrasound scans, maternal blood tests for conditions such as Down syndrome, amniocentesis, CVS and pre-implantation genetic diagnosis (PGD). ${ }^{27}$ Ultrasound scanning was found to be by far the most frequently encountered method of screening among the participants, with eighteen of the twenty women having been scanned one or more times during at least one pregnancy. ${ }^{28}$ Eight out of twenty participants had undergone a specific maternal blood test for Down syndrome and spina bifida (within New Zealand this test is referred to as Maternal Serum Screening, or MSS2). Four of the twenty participants indicated that they had not undergone any method of prenatal screening during at least one pregnancy. Of these four women, one had given birth to a baby with Down syndrome. This participant stated that she had not been offered any form of prenatal screening during this pregnancy. ${ }^{29}$

\footnotetext{
${ }^{26}$ Lethal fetal abnormalities include conditions such as anencephaly (partial or full absence of the head) and serious malformations of body structures and systems which are associated with inevitable neonatal death soon after birth (Ferreira da Costa et al, 2005). Such conditions are often clinically classified as being 'incompatible with life'.

${ }^{27}$ PGD involves the genetic screening of embryos and therefore is a method of prenatal screening which is only available to women undergoing in vitro fertility treatment (IVF), where embryos are cultured in the laboratory setting before implantation into the uterus. None of the participants within this study had undergone this method of screening; however two participants, along with their partners, had personally undergone genetic profiling subsequent to the birth of babies with genetic abnormalities.

${ }^{28}$ This finding is consistent with international data on the widespread use of ultrasound in routine prenatal care (see chapter two for further).

${ }^{29}$ It should be noted that this participant held a negative view of screening, and indicated that she did not regret the lack of opportunity to detect her child's condition prior to birth.
} 
As I will argue in this thesis, mainstream discourse on pregnancy care frequently presents prenatal screening as a reliable 'antidote' to the risk of fetal abnormality. However, several quantitative findings from this study appear to problematise this assumption, and personalize the issue of the technical shortcomings of this practice. ${ }^{30}$ Four participants had first-hand, ongoing personal experience of congenital genetic abnormality within their own family, having themselves given birth to children (still living) with non-lethal conditions. Each of these participants stated that they had undergone prenatal ultrasound screening prior to the birth of these children. In two of these cases, the relevant screening tests are not yet available within New Zealand as part of the standard 'package' of prenatal screening, and these conditions would not have been detected prior to birth in any case. Therefore, an important finding emerging from this study was that there was not a direct correlation between women's uptake of prenatal screening and the detection of fetal abnormality per se. While this observation is in fact well-recognised in the clinical domain, as will be argued later there appears to be a significant 'gap' in popular understanding of the scope and limitations of available screening methods.

Of the twenty participants, four had received diagnoses of fetal abnormality following prenatal screening. Of these four, two women subsequently underwent medical terminations, while the other two continued with the pregnancies.

I would suggest that the inclusion of a small amount of simple quantitative data within a qualitative study usefully highlights the diverse scope of pregnancy-related experience in a way which may not be acknowledged within clinical accounts of

\footnotetext{
${ }^{30}$ See chapter two for further.
} 
women's engagement with prenatal screening, and further signals the value of qualitative research in 'embodying' and qualifying statistics relating to women's 'uptake' of these technologies.

\section{Ethics approval and cultural considerations}

Ethics approval for the empirical component of the research was referred by the Human Ethics Committee of Victoria University to the Central Regional Ethics Committee (CREC) of the New Zealand Ministry of Health. The study design, interview questions and participant consent forms ${ }^{31}$ were subsequently approved and overseen by CREC until the completion of the data collection and analysis. ${ }^{32}$ As noted earlier, the study was not intended as a representative sample but rather an exploratory investigation, offering a platform for more specific future research within the New Zealand context. No claim is therefore made as to the representativeness of the participant sample in terms of variables such as socio-economic status and ethnicity. The means of participant recruitment did not target any specific ethnic group, however the means of recruitment created the potential for Maori participation in the research. In recognition of the special status of Maori as Tangata Whenua in accordance with the principles of the Treaty of Waitangi, informal consultation around a culturally appropriate approach to potential Maori involvement in this research was undertaken with Victoria University’s Maori Research Advisor (Tai Walker). Ms Walker advised that in the event that one or more participants identify as Maori and wish to discuss issues of cultural sensitivity, I should approach her again for further advice. The participant information sheet indicates that Maori participants wishing to address such issues prior to involvement in the study should indicate this to

\footnotetext{
${ }^{31}$ See appendix 6.

Study approval reference number CEN/05/08/056.
} 
me. Three participants identified as Maori, however none of these women indicated a need for further consultation. One participant identified as Pacific Islander, and stated that she did not require me to undertake consultation with a Pacific Island advisor.

Given the paucity of research on Maori and Pacific maternal health in New Zealand, it is hoped that this research provide a starting point for specific investigation of the experiences and views of Maori and Pacific women.

\section{Overview of chapters}

Chapter two considers the orientation of contemporary prenatal care in terms of material practices which shape women's screening decisions, including the representation of these practices by clinicians and within public health material. The chapter traces the trajectory of prenatal care from a targeted to a routine practice within the last three decades, and establishes the pivotal role of pregnancy ultrasound in this shift.

Chapters three and four draw specifically on the interviews undertaken for this research. Chapter three explores key themes in women's experiential accounts. The chapter aims to make available a 'snapshot' of a range of previously hidden experience within the New Zealand context.

Chapter four explores the moral framework of women's screening decisions, focusing on the 'moral work' involved for individual women in this process. The discussion identifies a number of themes which variously illustrate the nature and personal significance of the private 'work' involved in screening decisions. 
Chapter five considers the ethical implications of current practice. This includes an evaluation of the ethics of recent trends to constitute prenatal screening as an activity of public health. The chapter focuses specifically on the issue of informed consent, and includes a case study which highlights tensions between the principle and 'lived reality' of this notion.

Chapter six grounds the topic within a local context, over-viewing the evolution of current screening practices within New Zealand. The chapter considers factors specific to New Zealand which influence women's screening decisions, including the emergence of a trend to constitute screening as a public health activity. Discussion includes consideration of public consultation over emerging screening technologies within New Zealand.

Chapter seven explores the significance of the visual nature of prenatal ultrasound scanning in terms of women's experience, as well as in relation to the broader medical and popular embrace of this technology. The discussion also considers the relationship between currently emerging 'visualist' technologies and evolving medical and social expectations around the nature of contemporary pregnancy care.

Chapter eight's discussion reprises the critique of 'choice' which runs throughout the thesis, reiterating the argument that such decisions are discursively and materially constrained within the context of contemporary understandings of what constitutes 'standard' prenatal care. This chapter considers the potential to reconfigure current practice in light of the screening experiences considered in this thesis, drawing on these accounts in proposing a number of pragmatic changes to current practice in New 
Zealand. To conclude the thesis, the discussion highlights participants' attitudes towards the place of public consultation as an ethical safeguard in determining the direction of prenatal screening programmes, and reflects on evidence of a recent and marked shift in attitudes towards public engagement in bioethics decisions in New Zealand within the current political climate. 


\section{Chapter Two \\ From 'high risk' to mainstream: the shift to routine pregnancy screening for fetal abnormality}

\section{Introduction}

As the most widely-used method of prenatal screening in the world, obstetric ultrasound is firmly established within both medical and mainstream popular discourse as a necessary and desirable element of contemporary pregnancy care. As Riddle (2005) notes, the conjunction of both medical and social expectation can be seen to have constituted prenatal ultrasound as a technology which is at once 'normal' and normative. A number of studies confirm the generalized popularity of ultrasound amongst pregnant women, ${ }^{33}$ highlighting in particular social expectations around pregnancy as an experiential 'commodity' 34 .

Both as a medical and a cultural 'phenomenon' the popularity of prenatal ultrasound warrants examination, not least because this technology is recognized as having played a pivotal role in the broader public acceptance of other methods of screening pregnancies for fetal abnormality, and subsequent trends towards the routine implementation of prenatal screening programmes generally. As McNay et al (1998) note, until as late as the 1970s when ultrasound became widely available, the notion of diagnosing fetal abnormalities prior to birth was largely unknown within obstetrics. Yet prenatal ultrasound now constitutes a part of the routine care of pregnancy for women in most Western countries (Garcia et al, 2002). As this chapter will show, both the rapid ascendance of prenatal ultrasound as the most widely used screening

\footnotetext{
${ }_{34}^{33}$ See for example Press \& Browner, (1995 and 1997).

${ }^{34}$ At least this is the case within Western industrialized countries.
} 
technology, and its current 'routine' use, raise a number of significant clinical and social issues which are yet to be addressed in the context of contemporary practice.

In accounting for the current widespread use of prenatal ultrasound, theoretical analyses have frequently focused on the significance of its visuality in shaping both social attitudes towards and individual engagement with this technology (Petchesky 1986, Taylor 1998, Weir 1998a, Zechmeister 2001), a phenomenon which is considered in more depth in chapter seven. As a prelude to that discussion, this chapter reviews and discusses the relevant literature on pregnancy ultrasound, and considers the clinical rationale for current practice. This chapter also establishes the clinical context of participants' experiences, and therefore constitutes necessary groundwork for the analysis of empirical data in chapters three and four. This technical background information is useful to come to terms with the entwinement or ‘intertextuality' of medical and cultural texts which characterize mainstream discourses of prenatal screening (Weir, 1998b).

Exploring the medical constitution of ultrasound as a routine part of contemporary prenatal care, this chapter raises a number of questions about the implications for women's decision-making and control over their pregnancies.

\section{What is ultrasound?}

In prenatal ultrasound, a transducer is passed over the abdomen of a pregnant woman and the reflected sound waves produce a two-dimensional, real-time image of the fetus on a monitor. The clinical purpose of this technique is to allow visual assessment (by either a sonographer or obstetrician) of the development of anatomical structures, and to evaluate possible indications of abnormality (Nyberg et al, 2003). 
Within clinical literature, the visual data obtained during an ultrasound exam is considered useful as a means to accurately date the gestation of the pregnancy, which is clinically helpful both to avoid unnecessary induction of labour for post-term pregnancies, and to determine the safest method to terminate a pregnancy at a given stage of pregnancy (Cunningham, 2005). Visual imaging is also considered useful to help establish the position of the fetus, for example where a breech presentation is suspected, and confirm the presence of a multiple pregnancy (Nyberg, 1990). Prenatal ultrasound is also used specifically as a means of screening for fetal abnormality. In addition to detecting a range of developmental anomalies (which may or may not be associated with genetic abnormality), prenatal ultrasound is commonly used to identify signs of a number of specific chromosomal disorders, including Down syndrome, cystic fibrosis, spina bifida and polycystic kidney disease (Cunningham et al, 2005). As ultrasound is medically defined as a method of screening rather than diagnosis, indications of abnormality detected during scans are generally followed up through further tests, such as amniocentesis, in order to obtain a result which is considered clinically diagnostic (Raynor, 2003).

As a method of screening for fetal abnormality, prenatal ultrasound is functionally linked to the selective termination of pregnancy. The detection of Down syndrome in particular represents a key focus of prenatal ultrasound screening protocols (Cunningham et al 2005). It is difficult to access data on rates of pregnancy termination undertaken for specific fetal abnormalities as published material is limited; in particular, there appear to be few up to date inter-country comparisons available. ${ }^{35}$ However a 1999 review of available per-country data on reasons for

\footnotetext{
${ }^{35}$ Stone and Austen (2006) note that no national records of such figures are currently kept in New Zealand.
} 
termination $^{36}$ indicates extremely high rates of termination following diagnosis of Down syndrome, the authors noting an average rate of 92\% (Mansfield et al, 1999).

Also referred to as Trisomy 21, Down syndrome is one of the most frequently occurring non-lethal chromosomal disorders in humans, with an incidence of one in 800-1000 newborn babies (Cunningham et al, 2005). Abnormalities associated with this condition include intellectual impairment, heart and intestinal disorders, increased incidence of leukaemia and hormonal disorders (Wigglesworth \& Singer, 1998).

\section{Overview of the development of obstetric ultrasound}

Ultrasound has been widely used in obstetrics and gynaecology as a diagnostic aid since the 1960s, its use now generally regarded within medicine as an indispensable aspect of the management of abnormal pregnancy (Cunningham et al 2005, Nyberg et al 2003, Filly et al 2002). However, its routine use for fetal screening during uncomplicated pregnancies is a recent phenomenon, dating as Harris et al (2004) note from the 1980s by which time scans were routinely offered to pregnant women in a number of European countries, a trend followed in recent years in the United Kingdom, Germany, Canada and the USA. Similarly, within New Zealand while no co-ordinated screening programme exists the majority of practitioners refer women for ultrasound screening as a standard element of prenatal care (Stone \& Austen, 2006).

Developed originally as a technology for military use during WWI, ultrasound scanning technology was modified for use within an obstetric setting b b

The authors note the lack of published data on termination rates per condition as a limitation of this review. 
Ian Donald in 1957 (Oakley, 1986). Prior to this, x-ray (fetal radiography) had been used in obstetrics to produce still images, used chiefly as a means of establishing and/or charting the gestational development of fetuses. For a time, medical enthusiasm for fetal radiography in the UK and USA was such that its routine use was unreservedly promoted within medical literature, ${ }^{37}$ and for several decades women presenting for antenatal care were routinely referred for fetal radiographs (Oakley, 1986). As the long term health risks associated with gamma radiation (in particular, childhood cancers) came to be recognized, fetal imaging by ultrasound came to be used in preference to x-ray. At first fetal ultrasound, like x-ray, was used primarily as a tool for tracking fetal development, and as Draper (2002) notes, scans for fetal abnormality were originally offered primarily in cases where clinicians suspected a problem, or to women considered to be at high risk due to factors associated with fetal abnormality (such as advanced maternal age or family history). However by the 1970s its use had extended to include the detection of congenital abnormalities, the expanding applications of fetal ultrasound mirroring a concurrent blossoming of new reproductive technologies, including specialized techniques of fetal medicine (Petchesky, 1986). By the 1980s ultrasound was regarded by many obstetricians as a necessity of the modern obstetric care of both normal and abnormal pregnancies, evident in the following statement:

Ultrasound is now no longer a diagnostic test applied to a few pregnancies regarded on clinical grounds as being at risk. It can now be used to screen all pregnancies and should be regarded as an integral part of antenatal care. (Campbell \& Little, 1980, cited in Oakley, 1986:7)

\footnotetext{
${ }^{37}$ See for example Speidel, E. and Turner, H.H. (1924). 'The Roentgen Ray Diagnosis of Normal and Abnormal Pregnancies', American Journal of Obstetrics \& Gynecology, 7: 697-702, as cited in Oakley (1986).
} 


\section{The safety of prenatal ultrasound}

A review of clinical literature on ultrasound clearly suggests a generalized medical view of the safety of the technology (an attitude similarly reflected in mainstream popular accounts). Cunningham et al's (2005:390) assertion that there are "no confirmed damaging biological effects in mammalian tissue in the frequency range of medical ultrasound" is typical of appraisals of the safety of ultrasound to be found in clinical textbooks. As Oakley (1986) and Wagner (1994) have argued, this position appears to rely significantly on the absence of demonstrated negative effects, rather than on evidence of clinically proven safety. While such texts often recognize that few clinical trials have in fact been undertaken to evaluate the long-term safety of ultrasound, ${ }^{38}$ Wagner (1994) suggests that an apparent lack of clinical interest in undertaking such research reflects a broader reluctance to confront clinical reliance on ultrasound in Western obstetrics.

Those studies which have evaluated the safety of ultrasound during pregnancy conclude that while the technology is not definitively associated with negative effects, ultrasound does produce physical changes or 'bio-effects' in fetal tissues (Dinno et al, 1989). The most recent (1998) study notes an increased incidence in left-handedness in children having been exposed to fetal ultrasound, suggesting associated long-term neurological impacts (Kieler et al, 1998). Prior to these findings, a correlation between repeated exposure to ultrasound and fetal growth retardation had been demonstrated by Newnham et al (1993), leading the study's authors to recommend restriction of ultrasound to use only where clinical necessity was indicated.

Official protocols governing the practice of ultrasonography ascribe limits for appropriate use and similarly emphasize the principle that ultrasounds should be

\footnotetext{
${ }^{38}$ See for example Newnham et al, 1993 and Chamberlain et al, 2001.
} 
performed in pregnancy only when medically necessary. ${ }^{39}$ Such guidelines do appear to acknowledge the potential for damaging effects; for example, the American Institute of Ultrasound in Medicine (AIUM) states that when performing ultrasound, clinicians should use "the lowest possible exposure setting to gain necessary diagnostic information" (AUIM:1116). However, it is well recognized within obstetric literature that the vast majority of clinicians now offer pregnancy ultrasound on a universal basis (Filly et al, 2002). Furthermore, the development of newer forms of high-resolution imaging (e.g. NTS and 3D scanning) have seen a relaxation of guidelines limiting the intensity of ultrasound exposure; a U.S. report by the National Council on Radiation Protection (NCRP, 2002) notes that the permissible intensity setting for prenatal ultrasound is now eight times higher than protocols set prior to 1991.

Within some feminist critique (for example the position taken by FINRRAGE ${ }^{40}$ ) the field of reproductive medicine has often been criticized for a 'gung-ho', pioneering attitude towards the implementation of new techniques, and a degree of contempt for measures intended to restrict innovation (e.g. on ethical or social grounds). It may be suggested that such a critique is somewhat borne out in the fact that the widespread implementation of pregnancy ultrasound appears to have occurred in advance of rigorous evaluation of either utility or safety, and in comments such as that of Chamberlain et al (2001) within a recent clinical textbook that "ultrasonographers themselves reject the research evidence about the effects of ultrasonography as outdated" (2001:412).

\footnotetext{
${ }_{40}^{39}$ See Nyberg et al, 2003 and Cunningham et al, 2005)

${ }^{40}$ Feminist International Network of Resistance to Reproductive and Genetic Engineering.
} 


\section{Clinical reservations relating to prenatal ultrasound screening}

While clinical concerns about the use of obstetric ultrasound have rarely entered the public domain, there has nonetheless been an element of ongoing internal debate within obstetrics and medical ultrasonography on the clinical limitations of ultrasound as a screening tool. In particular this has related to questions about the value of the routine (as opposed to targeted) scanning of pregnancies for fetal anomalies, and this will be considered later. However a number of other concerns emerge in the clinical literature which relate to technical limitations of ultrasonographic technology itself. Given the medical valuing of ultrasound as an indispensable element of modern prenatal care, it seems worthwhile evaluating the statistical evidence relied on to support clinical claims. In examining statistical measurements of the diagnostic sensitivity of ultrasound, some limitations become evident in association with its use as a broad-based screening tool. Cunningham et al (2005:322) note that "sonography can identify at least 35 to 50 percent of major fetal malformations". The rate of nondetection implied by these figures would perhaps surprise many lay people, and in particular pregnant women who presumably participate in screening on the assumption that the technique is clinically reliable. In fact, within specialist clinical literature, questions relating to the technical accuracy of ultrasound-based assessments constitute an ongoing point of concern, with the phenomena of both missed and misdiagnosis widely recognized as endemic clinical limitations. In spite of popular assumptions about the efficacy of ultrasound as a screening tool, there appears to be considerable scope for error in the visual assessment of fetal structures, and an ostensibly 'normal' scan may in fact constitute a 'false negative' result, whereby a fetal anomaly is not detected. As Ewigman notes, the use of ultrasound to detect fetal abnormality in low-risk pregnancies remains particularly controversial, due to the rate at which sonographers fail to detect abnormalities when they are present, a fact 
highlighted by the RADIUS study which found that out of 16,000 low risk women scanned before 24 weeks, only $17 \%$ of major abnormalities were detected (Ewigman et al, (1993) cited in Cunningham et al, 2005). As Cunningham notes, "It appears that, even under ideal circumstances, the detection rate for major anomalies may not be high enough to warrant screening low-risk women" (2005:326). In addition to problems with sensitivity (i.e. detection rates), ultrasound-based screening is associated with a limited 'specificity', ${ }^{41}$ resulting in a high incidence of 'false positive' diagnosis. $^{42}$

Problems with the sensitivity of ultrasound relate significantly to its historic use as an opportunistic screening technique, which applies a 'catch-all' approach to detecting fetal abnormalities. As Wald et al (1998:173) observe, "there are few areas in medicine where a test involves examining everything that can be seen or measured"; and the scope for 'accidental' discovery of an array of abnormal findings during the course of a routine pregnancy scan should certainly be recognised as distinguishing this practice from other methods of medical screening. However, it is significant to note that newer ultrasound techniques, which do target specific signs of abnormalities, also have high failure rates for detection. ${ }^{43}$ Other factors are known to confound the sensitivity and specificity of ultrasound. Clinical evaluations based solely on the interpretation of visual images raise certain technical difficulties. As Nyberg (1990) notes, in some cases benign variations in normal structures are misinterpreted as abnormalities; for example, an unusually large colon in a normal fetus may look identical to an abnormal mass. Furthermore, it is clinically recognized that a

\footnotetext{
${ }_{41}^{41}$ 'Specificity' refers to the capacity to accurately distinguish between a variety of possible findings.

${ }^{42}$ That is, diagnosis of an abnormality where none is present.

${ }^{43}$ See subsequent discussion on Nuchal Translucency Scanning (NTS), a more recently developed method of fetal ultrasound screening used specifically to search for signs of Down syndrome.
} 
significant number of normal fetuses exhibit abnormal growths such as cysts in the brain and lungs at certain stages of development, which resolve spontaneously later in the pregnancy. For example, Nyberg (1990) notes that choroid plexus cysts ${ }^{44}$ occur in up to $2.5 \%$ of all fetuses, and usually resolve by 24 weeks' gestation. However, as these cysts are also sometimes associated with chromosomal abnormalities, they are viewed by sonographers as 'markers' for diagnosis. Such examples highlight the reliance of a visually based technology on the discretion of individual sonographers in weighing the likelihood of a chromosomal abnormality against the risks associated with subsequent diagnostic tests (e.g. the risk of miscarriage associated with amniocentesis).

It is also of note that obstetrics and sonography textbooks themselves typically do not address the clinical limitations of ultrasound in significant depth. The 'reassurance' value of ultrasound is often foregrounded, in a way which it could be argued does not draw specific attention to problems such as the propensity for false diagnosis: The following comment from a sonography textbook illustrates this:

The fetus is examined quite literally from head to toe. In the vast majority of cases, the fetus appears normal and parents can be reassured regarding the health of their baby. (Nyberg et al, 2003:1)

As clinicians themselves are both the authors and consumers of such texts, this apparent 'blind spot' for the demonstrated clinical shortcomings of diagnostic imaging seems to resonate with Press and Browners' (1994) contention that practitioners themselves are somewhat complicit in the popular 'fiction' of prenatal screening as a tool of reassurance.

\footnotetext{
${ }^{44}$ Fluid-filled cysts of the brain.
} 


\section{Issues around practitioners' role: technical competence and the question of patient counselling}

As suggested previously, the accuracy of ultrasound screening is directly (although not exclusively) linked to the proficiency of sonographers (NSU, 2007). The expertise and experience of sonographers is increasingly acknowledged as a critical issue in addressing deficits in the clinical efficacy of ultrasound, with variations in levels of operator competence known to have a measurable impact on the rate of detection of fetal anomalies (Filly et al, 2002). ${ }^{45}$ The question of operator competence and professional standards has increasingly led to calls for the standardized and ongoing accreditation of sonographers. ${ }^{46}$ Such measures occur within the broader context of the increasingly litigious environment of medical practice, and in particular the emergence of malpractice claims for 'wrongful birth' and 'wrongful life' ${ }^{47}$ The issue of medical liability is particularly salient within the US context ${ }^{48}$ where as Cunningham et al note $75 \%$ of obstetricians have been sued at least once during the course of their career (2005); however, the phenomenon of obstetricians and midwives practising 'defensively' is also now increasingly recognised within the Australasian context (Cunningham et al 2005, Brodie, 2006).

As the question of patient autonomy and in particular the right of informed consent increasingly shapes medical policy, the provision of adequate information by

\footnotetext{
${ }^{45}$ As highlighted in the RADIUS trial, where the most experienced sonographers detected up to $35 \%$ of abnormalities, as opposed to the least experienced, who found as few as 13\% (see Ewigman, 2003).

${ }^{46}$ For examples within the New Zealand context, see Stone \& Austen (2006).

${ }^{47}$ A claim of 'wrongful birth' is typically brought by parents of a child born with a congenital abnormality, who argue that the obstetric practitioner failed to provide them with adequate information about prenatal screening options, thereby depriving them of the opportunity to terminate an affected pregnancy. A claim of 'wrongful life' is usually brought on behalf of the affected child, and is a tort which is to date largely unrecognized within most legislatures (Capen, 1995).

${ }^{48}$ Within the UK, medical negligence suits are also increasingly recognized as contributing to an environment of defensive practice, with $70 \%$ of this type of litigation relating to obstetrics (Johanson et al, 2002).
} 
practitioners is recognized as another issue of concern in the practice of prenatal ultrasound. Laynyg (1998) notes that inadequate counselling of pregnant women undergoing Nuchal Translucency Scanning (NTS) ${ }^{49}$ to detect Down syndrome is a serious problem with current practice in the UK. Garcia et al (2002) cite a number of UK studies which indicate several problems with the provision of information about screening by clinicians, including the widespread failure to inform pregnant women about the clinical purpose of the procedure, and inconsistent levels of practitioner knowledge. Such concerns are similarly reflected in a recent New Zealand report on screening for Down syndrome, which cites poor practitioner knowledge of newer techniques such as NTS and inconsistent patient counselling as a significant concern (Stone \& Austen, 2006). ${ }^{50}$

In spite of these clinically recognised concerns, the implementation of routine prenatal ultrasound continues apace worldwide, with routine scans now officially endorsed in the UK (Williams et al, 2005) and Germany (Nyberg, 1990), Sweden, Norway and Iceland (McNay \& Fleming, 1998), Canada and Australia (Harris et al, 2004). Within New Zealand pregnancy ultrasound is, in effect, universal. Routine scans are not officially endorsed by the Ministry of Health and there is currently no nationally coordinated screening programme; nonetheless prenatal ultrasounds are publicly funded for all pregnant women, and NTS screening is recognized as being widely utilized and offered on an ad hoc basis (Stone \& Austin 2006, NSU 2007). ${ }^{51}$

The origins of and impetus for this shift towards routine prenatal ultrasound screening will now be considered.

\footnotetext{
${ }^{49}$ NTS is discussed more fully below.

${ }^{50}$ See chapter six for a discussion of current prenatal screening practice in New Zealand.

${ }^{51}$ See chapter six.
} 


\section{The trajectory of routinisation}

As noted earlier, within the field of obstetric sonography, there is evident professional disagreement about the clinical value of routine pregnancy scanning. Indeed, as Filly \& Crane (2002:713) note "there is intense debate about the appropriateness of routine obstetric sonography in the United States, and knowledgeable practitioners have taken both sides of the argument" (2002:713). Such debates highlight a key tension underpinning contemporary practice, that is, an apparent mismatch between official policy and day-to-day prenatal care as it would seem to be being conducted in the clinical setting. This disparity was first observed in social scientific literature by Petchesky (1986), and is also recognized within clinical literature (Dooley 1999, Wald \& Kennard 1998, Filly \& Crane, 2002).

The practice of universal pregnancy ultrasound scanning is not endorsed within a number of key clinical protocols which have traditionally directed international obstetric practice. Neither the American College of Obstetricians and Gynecologists (ACOG) nor the American Institute of Ultrasound in Medicine (AIUM) supports the practice of routine pregnancy ultrasound (Filly \& Crane, 2002). As noted earlier, the AIUM stipulates that ultrasound should be used in pregnancy only when medically indicated (2003, cited in Nyberg, 1990). Similarly, the World Health Organisation (WHO) takes a conservative view on the appropriateness of routine use, citing evidence that increased levels of surveillance of normal pregnancies are linked to accelerating rates of unnecessary medical intervention world wide. ${ }^{52}$

\footnotetext{
${ }^{52}$ See Wagner (1994) for a discussion of the relationship between WHO guidelines and current obstetric practice.
} 
While in the USA the ACOG recommends against routine pregnancy ultrasound, the most recently available figures (2001) show that $67 \%$ of all pregnancies are nonetheless scanned (Cunningham et al, 2005).

Taylor (1998) notes that prior to the mid-1980s, no significant evaluations had ever been undertaken to establish the clinical utility of routine ultrasound. In 1984 in the United States, the National Institute of Health $(\mathrm{NIH})$ reviewed the clinical benefit and risks of prenatal ultrasound, the published results concluding that there was no convincing evidence that the routine screening of low-risk pregnancies led to improvements in either perinatal morbidity or mortality (Filly \& Crane, 2002). The NIH then produced a list of pregnancy-related complications to direct practitioners in deciding under which circumstances the use of ultrasound was warranted. ${ }^{53}$ However as Filly \& Crane (2002) note, the use of routine ultrasound nonetheless continued to gain momentum, and in an attempt to resolve the question of appropriate use in uncomplicated pregnancies, the NIH commissioned a major clinical trial. The 1993 RADIUS trial (Routine Antenatal Diagnostic Imaging with Ultrasound), a large-scale study of 15,151 women undergoing low-risk pregnancies, concluded that there was no clinically significant benefit associated with the use of routine (as opposed to targeted) pregnancy scanning (Ewigman et al, 1993). Rather than settling the question of routine screening, such findings (and the results of subsequent studies claiming to refute the RADIUS conclusions) have provoked continuing debate within obstetrics and ultrasonography about both the purpose and clinical value of routine pregnancy ultrasound (Antsaklis 1998, Wald \& Kennard 1998, McNay \& Fleming 1998). Irrespective of the official clinical position, the practice of ultrasound use has

\footnotetext{
${ }^{53}$ See appendix 7.
} 
continued to shift from a focus on complicated pregnancies towards universal implementation (Taylor, 1998).

In accounting for practitioners' apparent disregard of official protocols, Williams' (2006) observations about the characteristic lag-time between the implementation and evaluation of reproductive technologies has considerable resonance for the evolution of prenatal screening. Filly \& Crane (2002) suggest that by the time attempts were made to regulate the use of prenatal ultrasound, many practitioners had made their own evaluations and wholeheartedly embraced the technology, often (at least within the USA) having already made considerable financial investment in implementing scanning within their own practice: as he notes, "by the time the NIH study results were published, the issue had already taken on a major financial impact in American obstetrics. Any result other than an overwhelming endorsement of routine sonography was certain to meet with a backlash" (2002:714). ${ }^{54}$ The following remark from an ultrasonography textbook would certainly seem to confirm an attitude of resistance within the clinical sphere to attempts to limit ultrasound use: "Although there are many indications for performing obstetric ultrasonography...we believe that being pregnant is reason enough" (Nyberg et al, 2003:1).

Ironically, the attempt to limit the use of ultrasound may in fact have established the conditions for its routine use. The NIH guideline produced to limit inappropriate use of ultrasound lists 28 indications where use is acceptable.$^{55}$ As Taylor (1998) has observed, the categories for inclusion are extensive and in some cases very broad,

\footnotetext{
${ }^{54}$ Presumably the 'financial impact' referred to here would relate to the cost of the ultrasound equipment, as well as perhaps the financial benefits associated with increased patient demand for the ${ }_{55}$ number of scans during a pregnancy within a 'user pays' healthcare setting.

${ }^{55}$ See appendix 7.
} 
with considerable scope for the inclusion of low-risk pregnancies (for example, the category for the "assessment of fetal wellbeing"). Furthermore, the guideline supports the use of ultrasound for the "estimation of gestational age for patients with uncertain clinical dates". Given that pregnant women frequently are uncertain of their exact date of conception, and indeed that the normal duration of pregnancy is recognized in terms of a range of between 38-40+ weeks (Bennett \& Brown, 1996), a vast majority of pregnant women would qualify for a scan according to this indication. In Petchesky's (1986) analysis, this endorsement of ultrasound use for generalised pregnancy dating effectively undermined official reservations about unlimited ultrasound use and has played a significant role in the routinisation of prenatal ultrasound within the United States (1986).

\section{Recent developments in prenatal ultrasound: Nuchal Translucency Scanning}

A comparatively recent innovation in prenatal ultrasound, Nuchal Translucency Scanning (NTS) has rapidly been integrated into practice and is now widely used in screening for Down syndrome (Malone et al, 2005). Developed in the 1990s as a screening test specifically to detect Down syndrome, the implementation of NTS has signalled a shift in focus for screening from the second to the first trimester of pregnancy (Williams et al, 2005). The technique is viewed as an innovation which retains the benefits of ultrasound as a non-invasive procedure, while avoiding the clinical risks associated with genetic diagnostic techniques amniocentesis and Chorionic Villus Sampling (CVS) ${ }^{56}$ The ability to offer this form of scanning earlier in pregnancy is seen as an additional benefit, as earlier detection of fetal abnormality

\footnotetext{
${ }^{56}$ See glossary, appendix 1.Both techniques require the needle extraction of fetal DNA, and carry an approximate $1 \%$ risk of miscarriage (Nicholaides et al, 2005).
} 
enables termination to occur earlier, minimizing risks for women which are associated with the procedure (Nicholaides, 2005). ${ }^{57}$

The diagnostic rationale of the NT scan derives in part from the clinical observation first noted in 1866 by Dr Langdon Down that people with Down syndrome commonly have a thickening of the skin on the back of the neck (Nyberg et al, 2003). Chang (2006) notes that in the fetus with Down syndrome, the physiological cause of this increased thickness is not definitively understood, but is believed to relate to the accumulation of fluid due to cardiac, venous or lymphatic abnormality. This increased thickness may be observed on ultrasound and in the early 1990s the measurement of this fold of skin (which appears on ultrasound as a translucent fluid-filled space referred to as 'nuchal translucency') was proposed as a basis of a method for screening all fetuses to detect possible signs of chromosomal abnormality during the first trimester. ${ }^{58}$ The NTS measurement is used in combination with a calculation of maternal age $\mathrm{e}^{59}$ and gestational dating to produce a risk estimate for Down syndrome (Pilnick et al, 2004). Although risk ratios for determining a 'high-risk' NTS result vary internationally, Chang (2006) notes that within New Zealand a 'high-risk' result is usually determined as a greater than 1:300 chance of Down syndrome, this being the criterion for referral for diagnostic follow-up involving genetic testing such as amniocentesis (2006). ${ }^{60}$

\footnotetext{
${ }^{57}$ Previously practice had been to undertake scans at 18-20 weeks, which was considered the optimal period to clearly visualize fetal morphology. Screening at this point in pregnancy is recognized as offering a limited timeframe for the opportunity to terminate for fetal abnormality prior to the date of fetal viability (i.e. currently 23 weeks) (Nyberg et al, 2003).

${ }^{58}$ See Nicholaides et al, (1992). See also appendix 8 for a comparative image of normal and abnormal nuchal measurements.

${ }^{59}$ The incidence of Down syndrome is known to increase in line with increasing maternal age (Nyberg et al, 1990).

${ }^{60}$ However, it should be noted that there are currently no national guidelines on provision of NTS in New Zealand, where recently concerns have been raised over inconsistencies in performing this procedure and the interpretation of results. See chapter six for further discussion.
} 
A number of international studies have evaluated the usefulness of NTS in detecting Down syndrome; owing to variation in results between these trials, ${ }^{61}$ I will draw on those studies cited within a recent New Zealand report reviewing national and international practice (NSU, 2007). This document reports that when used as a 'stand alone' screening method, NTS has a sensitivity (detection rate) of $85 \%$, which is to say that the scan will identify Down syndrome in $85 \%$ of pregnancies where Down syndrome is present. The false positive rate (i.e. incorrect positive indication of Down syndrome $)^{62}$ was reported at 20-25\%, with 20,000-25,000 women with unaffected pregnancies therefore subsequently being referred for unnecessary follow-up testing.

Combining NTS with biochemical screening (i.e. maternal blood tests which may indicate signs of fetal abnormality) is recognized as improving detection rates for Down syndrome, and decreasing the rate of false positive diagnoses associated with using NTS alone (Wald et al, 2003, cited in NSU, 2007). NSU (2007) reports that combining NTS with first-trimester biochemical screening produces a sensitivity (accurate detection rate) of $87 \%$, and a false positive rate of $5 \%$. However as observed by Stone \& Austen (2006) in a recent New Zealand report, while a combined method of screening for Down syndrome is accepted as best practice internationally, within New Zealand biochemical screening has not historically been funded, leading to a high use of NTS by maternity practitioners as the sole method of screening for Down syndrome. This is seen as having led to high rates of unnecessary referrals for amniocentesis, with associated miscarriages of normal pregnancies. Unlike many other countries where biochemical screening has traditionally been widely utilised,

\footnotetext{
${ }^{61}$ The evaluation of such data is complicated by variables which include small but clinically significant differences between trials. For example, where one trial may evaluate results from NTS carried out at 10 weeks' gestation, another may draw conclusions based on results from 11 or 12 weeks.

${ }^{62}$ See glossary, appendix 1.
} 
within New Zealand ultrasound screening was the original technique available and has consequently been the chief method out of which current practice has evolved (Stone \& Austen, 2006). ${ }^{63}$

In addition to the problem of high false positive rates, several other factors complicate the use of the NT as an accurate indicator of fetal abnormality. The nuchal thickness of all fetuses increases with age, and therefore an incorrect assessment of gestational age can result in an inaccurate risk assessment (Nyberg et al, 2003). The optimal time period for accurately measuring the NT is very specific - between 11 weeks and 13 weeks, 6 days (Chang, 2006). Additionally, the scale of measurement required to establish abnormality (carried out using digital 'callipers' transposed over the image on a computer screen) must be accurate to significantly less than a millimetre. A normal measurement is between 0 and $5 \mathrm{~mm}$, with an overlap of 2 to $3 \mathrm{~mm}$ between normal and abnormal fetuses (Nyberg et al., 2003). The potential for 'false positive' results due to practitioner error is widely reported in medical literature. ${ }^{64}$

Furthermore, sonographers must be able to distinguish nuchal tissue from various nearby anatomical structures. As Nyberg et al (2003) note, when visualised on ultrasound the amniotic sac has an appearance similar to nuchal skin, leading to the potential for incorrect or misplaced measurement. Furthermore, they note that in up to $10 \%$ of pregnancies the umbilical cord lies around the neck of the fetus, again leading to the potential for significantly increased (and therefore diagnostically misleading) measurements.

\footnotetext{
${ }^{63}$ See chapter six for further discussion of prenatal screening practice in New Zealand. ${ }^{64}$ See for example Stone \& Austin 2006, Nyberg et al 2003, and Williams et al, 2005.
} 
As discussed earlier, practitioner knowledge about appropriate use of NTS is another issue of concern. Within the New Zealand context, Stone \& Austen (2006) note considerable variation in practitioner knowledge of appropriate use of NTS, their survey finding that in spite of NTS being the most frequently offered method of screening for Down syndrome, $25 \%$ of surveyed NZ practitioners timed the scan incorrectly.

In recent social scientific work on contemporary prenatal screening practice, Pilnick et al (2004) and Williams et al (2005) observe that there is, as yet, little qualitative research on the social impact of the implementation and widespread use of NTS, including evaluation of pregnant women's experience of and attitudes towards this technique. In their analysis of NTS as an innovative modality of ultrasound screening, Williams et al draw parallels with Rapp's appraisal of pregnant women as 'moral pioneers' in the implementation of prenatal screening, arguing that increasingly highquality fetal imaging, offered at an earlier stage of pregnancy, intensifies pre-existing tensions in the constitution of pregnancy as both provisional and 'concrete'. Pilnick et al argue that since its introduction in the 1990s NTS has been presented as a method with considerable advantages (such as earlier diagnosis) and fewer shortcomings than other methods. They suggest that in framing NTS as a more sophisticated, 'cuttingedge' technique than existing methods, the broad logic of routine screening is consolidated within popular understanding, the notion of screening as a 'choice' now being characterised less by decisions about whether or not to undergo screening, than by choices between 'old' and 'new' methods. 


\section{Routinising ultrasound screening: the implications for pregnant women}

The widespread use of prenatal ultrasound has profoundly altered both the experience of pregnancy, and its clinical 'management'. Within this thesis it is suggested that this is due to two key reasons: issues arising from the visual character of the technology itself (see chapter eight) and, as considered in this chapter, the constitution of ultrasound screening as a routine part of the care of pregnancy.

As Williams et al (2005) have argued, the tendency within clinical literature has been to evaluate the impact of ultrasound primarily in terms of improved technical sensitivity and clarity of imaging, with consideration of the social and psychological impact tending to lag behind the implementation of innovative techniques. This discussion attempts to address this gap in the literature, and considers the implications of routininsed ultrasound for women's experience and control over their pregnancies, focusing on NTS as the most recently developed screening method to achieve 'routine' status.

\section{The phenomenon of incorrect diagnoses}

With the broadening in scope of ultrasound screening from a targeted to a universal basis it may reasonably be assumed that greater numbers of women are exposed to the diagnostic shortcomings associated with the technology, with material consequences in terms of both physical and mental health. As a now widely used method of detecting Down syndrome, NTS raises significant questions about the fit between prenatal screening as a medical project and the expectations of pregnant women.

The issue of misdiagnosis is a case in point. Based on figures discussed earlier, significant numbers of women now receive incorrect diagnoses - both false positive and false negative ${ }^{65}$ - for Down syndrome during their pregnancies. As Marteau et al

\footnotetext{
${ }^{65}$ That is, a result which indicates Down syndrome is not present, when in fact it is.
} 
(1992) suggest, the nature and extent of the psychological consequences of incorrect diagnoses have to date received limited attention in clinical literature.

It is well-recognised that women receiving a diagnosis of fetal abnormality experience considerable anxiety, ${ }^{66}$ with qualitative studies such as the work of Rapp (1999a) and Rothman (1986) highlighting the emotionally difficult experience of waiting for the results of follow-up tests, and decision-making around termination of pregnancy. With regard to the use of ultrasound specifically, the significance for women of having seen fetuses subsequently diagnosed with abnormalities is noted as a factor in subsequent psychological difficulties after pregnancy loss (due to either miscarriage or termination) (Black, 1992). However, as with Rapp's observations about the clinical 'invisibility' of women's accounts of refusing prenatal screening (1998), false positive and false negative diagnoses appear to constitute another largely hidden domain of experience.

The handful of studies which do evaluate the psychological impact of misdiagnoses indicate negative impacts for women in terms of adjustment when the diagnosis is corrected. In the case of false positive results, Marteau et al (1992) and Baillie et al (2000) found that even subsequent to receiving a diagnostic 'all clear', women experienced feelings of dissociation from the pregnancy, and high levels of anxiety about the health of their baby, which sometimes lasted well beyond the birth of a normal baby. In evaluating these findings, Marteau et al conclude that the persistence of maternal anxiety following a normal result may be significantly related to the fact that the concept of a false positive result is largely unrecognised within popular understandings of pregnancy screening (1992). Following a false negative diagnosis,

\footnotetext{
${ }^{66}$ See for example Statham \& Green, (1993).
} 
families must readjust to the unexpected birth of a baby with Down syndrome. Hall et al (2000) found that women having received a false negative diagnosis during pregnancy experienced increased levels of parenting stress, negative attitudes towards the affected child, and in some cases anger towards clinicians. A significant finding was that those who had received a false negative result had significantly higher levels of stress after the birth of the baby than women who had not received screening at all. As with false positive diagnoses, the authors found that the negative effects were significantly linked to popular assumptions that ultrasound screening is a 'fail-safe' method of screening (Hall et al, 2000).

In addition to any psychological and emotional impact, the receipt of an incorrect diagnosis has consequences in terms of women's decision-making. As discussed earlier, it may be difficult enough to interpret a NTS result even when it falls within a 'low-risk' range. Women receiving a 'high-risk' result face the choice of whether or not to undergo invasive testing; where this decision is based on an inaccurate result, normal (and presumably in many cases, wanted) pregnancies may be lost. The personal ramifications of such decisions are yet to be evaluated, and, at least within the New Zealand context, there is little clinical data available which might provide a platform for such an enquiry. While as Stone \& Austen (2006:5) note, no data is recorded in New Zealand on either detection rates for amniocentesis or subsequent decisions made by women, the authors nonetheless suggest that significant numbers of women are currently affected: "best estimates would suggest that more normal pregnancies may be adversely affected by amniocentesis than the number of Down syndrome fetuses detected". 
Methods of screening which occur early in the pregnancy (such as NTS) also have implications for women in terms of exposure to clinically unnecessary procedures. Down syndrome is associated with a high incidence of spontaneous miscarriage; Morris et al (1999) note that approximately $43 \%$ of affected fetuses alive at 10 weeks' gestation will subsequently miscarry (cited in Stone \& Austen, 2006). As NTS is typically carried out at around 11-13 weeks (Chang, 2006), significant numbers of women may subsequently decide to undergo terminations which may not have been necessary had testing occurred later. As McFadyen et al (1998) suggest, this phenomenon has additional implications related to the psychological impact of highresolution imaging, with women tending to view the scan primarily in terms of a first 'encounter' with their baby (1998). Within this context, the decision to terminate a pregnancy subsequently found to be normal seems likely to have additional negative consequences for women.

\section{Routine ultrasound screening and maternal anxiety around pregnancy as inherently 'risky'}

Routinised ultrasound also has consequences for pregnant women in terms of the consolidation of a risk-based approach within popular discourses of pregnancy and birth. A shift from targeted to population-based screening would seem to locate all pregnant women on a continuum of risk where the designation of either 'low risk' or 'high risk' negates the possibility of 'normal' pregnancy as a default category within pregnancy discourse (Williams et al 2005, Lupton 1999). The risk/probability ratio produced for each woman undergoing NTS signals an entrenchment of this conceptual model, and the universal offering of this procedure illustrates how readily a baseline presumption of risk may be constituted as the basis of normal pregnancy care. This is a matter of concern among practitioners working within a 'primary care' 
model, ${ }^{67}$ where the impact of widespread medical intervention is often seen as jeopardizing the view that pregnancy is, for the majority of women who experience it, a straightforward, 'normal' physiological event. The promotion of this view is seen as being tied to demonstrated positive outcomes within maternity care, and is the basis of the primary care model of health (WHO, 1985). As Reiger (2006) notes, among primary care practitioners such as midwives, the intensification of prenatal screening (among other medical interventions) is viewed as contributing to a 'crisis of confidence' in birth, as midwives direct increasing amounts of their energies within practice to counselling women with deep-seated anxiety and lack of confidence in their ability to experience normal pregnancies and births. Among such practitioners the institutionalisation of 'risk' (and consequent intensive fetal monitoring) is often seen as having had a measurable impact on rates of normal birth, and certainly clinical literature increasingly acknowledges substantive links between practices such as continuous fetal heart rate monitoring during labour and rising rates of caesarean sections worldwide. ${ }^{68}$

The widespread implementation of pregnancy scanning as a strategy of 'risk management' has further consequences in terms of the extent to which women experience screening as a 'choice'. As suggested by Lippman (1994) and Pilnick et al (2004), where strategies of risk management in pregnancy care are constituted as a normal experience, the logic of necessity may eclipse the notion of screening as a matter of choice. This seems to be borne out in the interview-based studies of Press \&

\footnotetext{
${ }^{67}$ Broadly speaking, this approach emphasizes a preventive approach to health care provision, for example through enabling low cost access to primary care services (such as midwifery and GP care) and addressing a range of social determinants of ill health, rather than an exclusive focus on episodic
} medical intervention secondary to the emergence of health problems (WHO, 2008).

${ }^{68}$ See for example Sandmire (1990) and Albers (2001). 
Browner (1997), which found that pregnant women often do not regard undergoing screening as a choice and account for their acceptance of screening primarily in terms of risk avoidance. The contradictory relationship between discourses of risk and choice in screening will be considered in more depth later in the thesis and will not be elaborated on here, however one further salient consequence of this tension is played out in the fact that the routinisation of prenatal ultrasound has likely played a key role in establishing the conditions for a broadening cultural acceptance of this practice as necessary rather than optional.

Is 'informed consent' for pregnancy ultrasound currently a reality? Lippman (1991) suggests that prenatal ultrasound is the method of prenatal screening which occurs most frequently without women's informed consent. In addition to framing compliance with screening as a strategy of risk management, women's readiness to accept scans may also be linked to the popular view of pregnancy ultrasound as a medically neutral, 'zero-cost' procedure (Pilnick et al, 2004). As suggested in Press \& Browner's $(1997,1999)$ qualitative studies, many pregnant women have an incomplete understanding of the medical rationale for prenatal screening and its functional link with selective termination of pregnancy, tending to view screening primarily in terms of 'reassurance'. With regard to NTS specifically, a difficulty for pregnant women in consenting to the procedure relates to the fact that NTS does not produce definitive results but rather probabilities of risk. The work of Pilnick et al (2004) and Williams et al (2005) raises questions about the ability of lay people to utilize such results as a meaningful basis for their decision-making. Furthermore, Ettore (2000) suggests that women may not be aware that a low risk estimate is not a guarantee of a normal pregnancy. Conversely, receiving a nonreassuring NTS result is also problematic, in that women may struggle to integrate a 
probability ratio within their own evaluations of the need for further testing (Pilnick et al, 2004). The risk may be that in finding a probability-based estimate an unsatisfactory basis for decision-making, women may wish to abdicate responsibility for screening decisions to the practitioners undertaking their care. Naturally, this would problematise the assumption of informed consent for screening method such as NTS.

This question around practitioners' role in directing women's decision-making has indeed been noted as potentially complicating the question of consent for prenatal screening in general. ${ }^{69}$ For example, in examining women's reasons for accepting screening, Press and Browner note (1997) that institutional support for screening programmes is the most significant predictor of women's participation (1997). A further finding was the fact that irrespective of the information provided by practitioners, pregnant women frequently regard the mere offer of screening as an authoritative endorsement.

Furthermore, as Taylor $(1998,2002)$ has observed, pregnancy is increasingly viewed in terms of consumption (including the consumption of specific goods and services); in enacting pregnancy as a 'commodified' experience she argues, pregnant women attach significant value to scans as a source of pleasure. As suggested in chapters one and seven, such pleasures are nonetheless entwined with cultural norms around appropriate maternal behaviour; and popular expectations about prenatal scans as a first and much anticipated opportunity for pregnant women to 'meet' and 'bond' with

\footnotetext{
${ }^{69}$ See for example Williams; Alderson \& Farsides (2002) for a discussion of the authenticity of 'nondirective' practitioner counselling.
} 
their fetuses may add a further dimension of normative pressure to undergo screening (Black, 1992).

As Lippman (1999) and Press \& Browner (1994) have argued, the validity of informed consent for prenatal screening rests significantly on the availability of alternative choices for women, including the option to decline screening or engage selectively with available technologies. As their research suggests, women confront considerable normative pressure to undergo screening, often expressing the view that it is easier to 'opt in' than to 'opt out' $(1994,1997)$. Consequently in addition to problematising the voluntary nature of participation in screening, pervasive cultural assumptions around the value of this practice tend to marginalise alternative responses, and women who wish to decline screening altogether may feel that this is neither a medically nor socially sanctioned option (Rapp 1998, Press \& Browner 1997, Williams, Alderson \& Farsides 2002). Hence the question of women's consent for pregnancy scanning can be seen to be complex and not readily contained within a straightforward analysis. The issue of informed consent will be discussed further in chapter five.

Within the interview-based data analysed for this thesis, ultrasound was the screening method described by participants as most frequently offered and undertaken, with all but two women having undergone at least one scan in the course of their pregnancies. The majority of these accounts concur with the case made earlier in the chapter that prenatal scans are widely represented by practitioners as an 'everyday' aspect of pregnancy care, rather than expressly described as an option. ${ }^{70}$ In reflecting on their 
experience, a significant number of women felt that they had undergone scans without being aware of the purpose and potential outcomes of the procedure, a fact which, significantly, was not necessarily attributed to any professional shortcoming on the part of practitioners responsible for their care. Rather, in accounting for their acceptance of screening, participants frequently drew on the rationale that they had accepted it simply because it was offered. As Kerry said:

Um, I didn't actually think about it very much at the time, it was just sort of offered, and I suppose though I did it the first time around so we got to see what the baby was like... but I didn't do it to look for any kind of abnormality or anything, I think they just did it to...that wasn't kind of the focus for it, I just did it because it was offered I suppose.

Such comments suggest the pervasive impact of routinisation on popular assumptions about pregnant women's role in decisions relating to their care, highlighting the way in which presentation of a procedure as 'routine' may dilute a sense of responsibility for active decision-making. The logic of acceptance is contained within the offer itself.

In accounting for women's acceptance of prenatal screening, and the role this may have played in the impetus towards routinisation, Press \& Browner (1997) drew on McKinlay's (1982) analysis of the characteristic trajectory of medico-technical innovations. In this model the implementation of new technologies and procedures is seen to occur in the first instance on the basis of promising preliminary evidence, the withholding of anticipated benefits from patients then rapidly coming to be viewed as unjustified. In line with this view, as Press \& Browner suggest the implementation of newly developed methods of prenatal screening have typically occurred well in 
advance of both in-depth clinical evaluation and consumer demand. This apparent 'rush to screening' clearly sits in tension with the popular assumption around medical practice as 'evidence-based' and underpinned by scientific rigor, and, as Petchesky (1986), Taylor (1997) and others have noted, is extremely significant in coming to terms with the evident mismatch between policy and procedure as the milieu out of which the practice of routine prenatal ultrasound has evolved. This chapter has thus positioned the shift to routinised pregnancy screening in terms of a potent structural/institutional determinant of women's screening decisions. In line with this analysis, this phenomenon would appear to be readily incorporated within a broader 'medicalisation' hypothesis concerning reproductive health care (Oakley 1986, Martin 1987, Corea 1985 and Wagner, 1994). ${ }^{71}$ However, as Petchesky (1986) first influentially argued, the widespread popular embrace of this technology cannot only be understood in terms of the general medicalisation of contemporary pregnancy care, but ought to be recognised as deriving significantly from the visual character of the technique and, specifically, the complex meanings attached to the image of the fetus as a fetishised 'cultural object'. ${ }^{72}$ As this and the previous chapter suggests, the processes by which practices such as prenatal screening have become part of the everyday landscape of pregnancy care reflect the entwinement of a variety of norms, both strictly 'biomedical' and popular.

The following two chapters focus specifically on the empirical component of this research, drawing on participants' experiential accounts of prenatal screening as a 'way in' to exploring the personal significance of this practice.

\footnotetext{
${ }^{71}$ In this analysis, healthcare (and in this case maternity care) is seen as subject to ever-increasing medical intervention and control, generally at the cost of both patient autonomy and quality of care. ${ }^{72}$ The significance of the specific visuality of prenatal ultrasound for current practice will be discussed in depth in chapter seven.
} 


\section{Chapter Three \\ Themes in the experience of prenatal screening}

Ontology is multiple and reality leaves us in doubt. (Mol, 2002:166)

There are a number of things that strike me as unbelievably stupid that an awful lot of people do that are considered absolutely fine. (Rona)

As I have argued in preceding chapters, prenatal screening needs to be understood as a complex phenomenon, and one which confronts women with a 'multiplicity of norms' (Mol, 1998). The extent to which such norms reflect the reality of women's experience is the focus of the following discussion. How do pregnant women themselves experience the tensions generated within mainstream accounts of prenatal screening? In what ways do they 'rise to' and respond to this experience in their decision-making? These were the questions which directed the empirical component of this thesis.

\section{Purpose of the empirically-based chapters}

The following two chapters focus specifically on the experiential accounts of women drawn from interviews conducted for this thesis. This aspect of the research was undertaken for two reasons. Firstly, to gain a snapshot of women's experience, and to identify what, if anything, individual women consider to be at stake in their engagement with prenatal screening. A secondary aim was to explore the ways in which women account for their screening choices, and to consider the various "moral frames of reference" utilized by women in their decision-making (Scully et al, 2004:1416). 
In focussing specifically on experiential accounts the following chapters attend to Rapp's observation that prevailing discourses of prenatal screening are characterised by the omission of a 'lived dilemma' (1998:68). Analysis of participants' accounts suggests that the rationalizing discourses of 'need' and 'choice' which underpin popular understandings of routinised screening do not acknowledge either the ideological or clinical tensions attached to screening as an embodied experience. Frank $(1995,1997)$ has argued that biomedical discourse largely fails to acknowledge patients' moral lives, a view which has considerable resonance for participants' accounts of experience within this study. In many cases these women articulated a sense that the materiality of their experiences could not be adequately accommodated within mainstream understandings.

Qualitative research methods derive from the belief that a sociological understanding of phenomena needs to be grounded in the meanings which are held by social actors themselves (Payne \& Payne, 2004). In line with this view, an attempt to 'capture' an experiential phenomenon is necessarily directed by participants' own accounts of meaning (Fereday \& Muir-Cochrane, 2006). In consideration of the highly personal and sensitive nature of women's experience in this area, an approach which arose from the data itself seemed most appropriate in reflecting the notion of women themselves as the most authoritative commentators, or experts, on the experience (Rapp, 1987). A theme-based approach therefore was selected as an appropriate way to structure data of this nature. The overall theoretical framework of the discussion takes its lead from a body of qualitative sociological research (and related work in the field of nursing studies) on experiences of health and illness within which analysis of 
interview material is frequently structured along the lines of emergent themes, while also situating individual accounts relative to broader cultural influences. ${ }^{73}$ In structuring the discussion of participants' accounts within these chapters, thematic categories needed to be fluid enough to allow for the complex entwinement of discourses which typically characterises accounts of prenatal screening; therefore, headings do not necessarily indicate discrete boundaries between themes.

\section{Scope of participants' experience}

As Rapp (1998) has argued, medical accounts of pregnancy tend to assume a homogeneity of experience. However, it should be noted that the twenty participants for the study recounted a diverse range of experiences around prenatal screening. ${ }^{74}$ In a number of cases, these experiences were deeply personal and traumatic in nature, and related to what might be considered specific clinical 'incidents', including the decision to undergo late termination (in two instances) ${ }^{75}$ for genetic abnormality, loss of a pregnancy after amniocentesis, the experience of a "false-positive" 76 diagnosis of Down syndrome, and a "false-negative" screening result ${ }^{77}$ subsequent to which a baby was found at birth to unexpectedly have Down syndrome. Another participant recounted the experience of what she regarded to be a missed opportunity for screening which would have detected her child's serious genetic abnormality, a diagnosis with additional clinical implications for herself and her partner in terms of future pregnancies. ${ }^{78}$ For those women whose accounts were not concerned with the

\footnotetext{
${ }^{73}$ For recent examples, see Scully et al (2006a, 2004). Williams et al (2005) also broadly follow this approach.

${ }_{75}^{74}$ See chapter one for a basic demographic profile of participants.

${ }_{76}^{75}$ I.e. occurring at 20 weeks' gestation or later.

${ }^{76}$ As noted in chapter two, a "false-positive" result is considered to occur when a genetic condition, such as Down syndrome, is incorrectly identified. Such a finding may then be the clinical basis on which the decision to have an amniocentesis or termination of pregnancy is based.

${ }^{77}$ A "false-negative" result occurs when prenatal screening (typically ultrasound, but in rare cases amniocentesis) does not detect an existing genetic abnormality.
} 
specifics of a clinical diagnosis per se, experiential themes typically revolved around either questions relating to the practice of screening within the clinical setting, or ideological tensions between personal belief and mainstream discourse.

The set of experiences described in this thesis should be viewed as a 'snapshot' which, while it cannot be regarded as necessarily representative, nonetheless reflects a diversity of experiences on a continuum which includes both positive and negative accounts. It seems useful at this point to include some general findings on participants' attitudes as these help to contextualise the accounts considered in the following discussion, and offer a preliminary suggestion of the ideological and experiential 'demography' from which individual women's views had emerged.

\section{General attitudes towards the experience of prenatal screening}

It is of note that the majority of participants regarded their experience of prenatal screening as in some way problematic, a fact reflected in the frequently stated wish to "make things better" for other women; this emerged as a significant motivation for participation in the study. Many participants appeared to regard their experience as a kind of 'watershed' moment within the context of their own lives, sometimes indicating that previously held attitudes had been questioned and even transformed as a result. Of the participants who had undergone screening in at least one pregnancy, three indicated that on the basis of their experience they would be likely to decline screening in any future pregnancies. A sense of a 'gap' between mainstream and personal accounts of screening emerged as a consistent theme, and in many cases a wish to put a human face to the experience was explained as a another key reason for wishing to take part in the research. 


\section{Views on termination of pregnancy}

While participants' personal beliefs relating to termination of pregnancy were not formally sought as part of the scheduled interview questions, due to the subject matter such views were readily identified in women's accounts. Views on the acceptability of termination of pregnancy expressed in women's accounts reflected a general 'prochoice' perspective in the majority of participants. In the course of the interview, all but one participant indicated that they supported the principle of access to termination of pregnancy for all women.

A number of participants had undergone termination of pregnancy outside the context of prenatal screening, i.e. for reasons not related to fetal abnormality. One participant indicted that she was opposed, on religious grounds, to abortion under any circumstances.

Of significance is evidence of a clear distinction between attitudes towards the ethical and personal acceptability of termination in general, and the matter of selective termination. Ambivalence around the acceptability of selectively terminating fetuses affected by specific genetic conditions, such as Down syndrome, represents one of the most consistent themes in women's accounts. This is the case even where participants held an overall positive attitude towards the value of prenatal screening and had considered their own experience of screening to be unproblematic.

In spite of a sense of discomfort about the ethics of selective termination, participants' nonetheless generally endorsed the right of individual women to terminate a pregnancy because of fetal abnormality. Frequently women stated that personal inexperience of disability meant that they did not feel in a position to judge those who 
chose to terminate pregnancies on these grounds. However, it is interesting to note that a considerable number of women indicated that they themselves would be highly unlikely to seek a termination on these grounds. This position was almost invariably explained in terms of personal acceptance of disability and/or ideological discomfort with the notion of eugenics, which was frequently viewed as linked to the practice of selective screening.

\section{Attitudes towards disability}

The sample reflects an overall view of acceptance of disability relating to genetic conditions, and participants' accounts frequently indicated a valuing of disability as a hallmark of social diversity. In an attempt to gauge attitudes relating to the provision of state support for disability, and notions of 'genetic responsibility' (Peterson \& Bunton 2002, Lupton 1995), participants were questioned with regard to their own willingness to contribute to such support through public taxation. Nineteen of twenty participants stated that they were happy to do so, whether or not prenatal screening had been undertaken by the mother of genetically affected children. One participant however stated that she would be uncomfortable making such a contribution in cases where prenatal screening had been declined and a child with a genetic abnormality subsequently born.

Overall, participants' attitudes towards disability appeared to be in tension with prevalent medical 'functional' evaluations (Ettore, 2002, Shakespeare, 1998). In particular, concerns about the specific targeting of Down syndrome as the primary focus of screening programmes suggested that public health screening policies may be somewhat out of step with lay opinion on this issue. Within the study, two participants had made the decision to undergo termination of pregnancy on the basis of fetal 
abnormality. It is significant that their accounts nonetheless incorporate a broad acceptance of disability as a societal 'good' within their experiential narratives. Findings such as these would seem to problematise the presumed equivalence between women's 'uptake' and wholesale acceptance of prenatal screening which typifies mainstream discourses of pregnancy care (Press \& Browner, 1997, Lippman 1991, 1994). While many women may indeed feel entirely at ease with both the rationale and experience of screening, as this thesis observes, a significant body of ethnographic literature in this area clearly establishes ambivalence as a consistent meta-theme in pregnant women's experience of this practice. This sense of 'mixed feelings' is no less evident in women's account within this study, as the discussion below will show. It seems to be the case that, at present, public health discourse and policy in this area is yet to allow a discursive 'space' for women's evident ambivalence around this practice and its broader social significance. As chapters five and six will argue, the representational politics of prenatal screening raise a number of broader questions about the extent to which current public health policy can reasonably claim, as it increasingly does, a consultative, 'consumer-led' approach to the ethical use of emerging screening and reproductive technologies.

\section{Key themes in participants' experience}

The following discussion is structured in terms of the key experiential themes which emerged in the interviews undertaken for this thesis:

\section{Reassurance and control}

Screening and pleasure

"Treating a heavy thing as a light thing": the presentation of screening by clinicians

Experiencing risk 
Insufficiency of information

"The right not to know"

Acceptance and surrender

Disembodiment and provisionality

\section{Reassurance and control}

Participants frequently occupied contradictory positions in their view of pregnancy as either normal or 'risky' and this ambivalence was accordingly reflected in their evaluation of the reassuring potential of prenatal screening. Anna's response expresses the sentiments of many participants:

I would have liked to have stopped it, but then you know you're also half on it. So you're wondering well, maybe there's something I need to know, like it might die and I don't know about it. Know what I mean?

Melissa seemed to feel similarly torn; while morally uncomfortable with selective screening, her account nonetheless conveys a sense of obligation to undergo screening as a project of health:

I'm aware that my feelings aren't particularly rational, and they're not particularly in harmony with each other if you know what I mean, but there's like you know this is rationality versus emotions and what you fear...I mean everyone wants a healthy outcome of their pregnancy I guess.

Such ambivalence reflects Lippman's conception of prenatal screening as a site where women attempt to negotiate contradictory discourses of fear and reassurance (1994).

As both of these extracts suggest, what may constitute reassurance in a medical, or as Melissa implies, "rational", sense may itself be in tension with personal evaluations of wellbeing. 
However, within several accounts an overwhelmingly positive view of screening was expressed, both in terms of perceptions of the broad value of the technology, and in terms of individual experience. Within these accounts reassurance was viewed as the primary benefit of screening. As Lupton (1999) notes, pregnant women often frame prenatal screening primarily as a means of reassurance rather than a diagnostic tool, tending to regard the purpose of ultrasound scans as a means of establishing that there is 'nothing wrong'. This notion is illustrated within Karla's account of her engagement with screening, in particular in the comment that she undertook screening "to know things are ok".

Karla's perspective can be seen to reflect a broader 'medical model' conception of pregnancy as inherently risky, within which intensification of medical management logically equates to improved health outcomes:

...it's only because of all the new technology and the new knowledge and everything else that has come about that, you know, that most babies are born ok.

Deb expresses a highly positive view of the use of medical technology in pregnancy and her account of routinisation is similarly linked to a rationale of medical and technical progress:

I think it probably is becoming far more routine, I mean you look at the quality of the scanners that are available now compared to what has been available. Like all technology it's getting better.

Karla describes her three pregnancies and births as "trouble-free". However, for her, pregnancy is clearly not viewed as normal but as an unpleasant and risky time, something, as she describes, to 'get through'. 
If I get pregnant again I'll be looking into all of the latest technology. To me birth isn't a natural thing, you need all the help you can get really, and if it's there, why not use it?

For several women, the reassurance value of screening was explicitly articulated in terms of a clear wish to have the opportunity to terminate a pregnancy if abnormality was found. For example, Emily stated that she had no sense of dilemma about the purpose and value of screening: "I suppose I've always been able to think of it as quite cut and dried". Hence, it is important to note that while in a minority within the participant sample, a number of women were unambivalent in their evaluation of screening as a vehicle of choice and reassurance.

Where the reassuring potential of technology was perceived as highly valuable in this way, the capacity to obtain the maximum possible information was also typically regarded as a chief benefit of screening. Within this perspective, information is welcomed as a 'tool', which enables a more positive pregnancy experience. As Emily stated:

I think once you've had that you wouldn't change your mind and go back to not wanting to know. I suppose it's sort of self-perpetuating, it's like knowledge becomes your tool and your aid for feeling good, and having a good pregnancy I suppose.

In line with this view, the notion that information might be regarded as unwelcome or burdensome makes little sense. For Karla, a decision to refuse screening was viewed as questionable and perhaps morally irresponsible:

Well I've got this view that it's unfair, if you know that there's something wrong with that baby, and it's going to be abnormal, or you know, in a wheelchair or whatever that it's really unfair to bring that child into the world knowing that you know that. 
In line with this view, Karla questioned the entitlement to state support of women who proceeded with pregnancies after abnormal diagnoses had been made:

No, not quite so happy, not when they know definitively that there's something wrong, particularly if there's something major I think, well, if they're going to bring that child deliberately into the world knowing that, well then they should pay for it.

\section{Scans as a source of enjoyment: "it's just nice to see your baby"}

The fact that women's acceptance of screening is significantly motivated by the pleasure associated with the visuality of ultrasound scans is strongly suggested in literature on women's engagement with prenatal technologies (Petchesky 1987, Draper 2002, Harris et al 2004). ${ }^{79}$ Widely viewed as a standard part of pregnancy care, such scans have come to be popularly regarded as an integral and positively anticipated aspect of the embodied experience of pregnancy (Harris et al, 2004).

While a sense of ambivalence around the value of ultrasound scans was reflected in a considerable number of participants' accounts, a number of women nonetheless placed a high value on the pleasure they had experienced. As Melissa explained, "I loved having scans. I don't think that's the reason for doing it, but it is a reason for doing it". For some, the evaluation of the benefits of scans suggested an unproblematic splitting of diagnostic and social aspects of screening ${ }^{80}$ as Diane notes, "I like to see the baby, and I guess that's a social thing". Similarly for Emily, the experience of the scan itself is seen as inherently pleasurable, irrespective of outcome, and something she would recommend to every pregnant woman. For her, this pleasure appears to be at least as valuable as any diagnostic capability:

\footnotetext{
${ }^{79}$ The significance of the visual nature of ultrasound for screening practice and experience will be discussed further in chapter seven.

80 'Social', as opposed to strictly medical, uses of ultrasound are considered in chapter seven.
} 
...I'd be saying yeah have a scan it'd be really nice for you whether or not your baby's fine and if it's not that's ok, we'll talk about it then, but if it is yeah then it's great. So I would advocate it to people.

For Petra, seeing her baby during an ultrasound scan seemed to represent a moment of relief and resolve, as a point at which she was able to extricate herself from a prior position of dilemma following an apparently 'high-risk' result:

Anyway, this little thing, it kind of just waved at us, and I thought oh we'll live with Down syndrome, we thought we couldn't but we can.

Williams et al (2002c) note that many women perceive the nuchal fold scan as a noninvasive method of screening, tending to equate the notion of 'non-invasiveness' with a view that there is less at stake in terms of the information the test may provide. This concurs with Lippman's (1994) findings about the commonsense understanding of the notion of 'reassurance' as the primary function of prenatal screening. As the nuchal fold scan is often represented to women as a routine scan, as an indicator of normal growth and development, rather than a specific assay to detect signs of Down syndrome, women may be completely unprepared when a genetic abnormality is indicated. This perception of the nuchal fold scan as a non-invasive, standard (and therefore unconcerning) part of care was also reflected in participants' accounts in this study.

\section{"Treating a heavy thing as a light thing": attitudes towards the representation of prenatal screening}

Almost without exception, participants described ultrasounds (including NTS, where this had been undertaken) as having been presented by clinicians as a standard part of their antenatal care. For a number of women this was not in itself a matter of concern, and, unsurprisingly, those who appeared to identify with a 'medical model' perspective on childbirth tended to view the routinisation of prenatal screening as 
emblematic of progress in maternity care, and to equate this with optimal choice and control. However, the majority of participants expressed various degrees of concern about how appropriately and/or accurately screening had been presented to them by their caregivers. Typically this was recounted as not having been a matter of concern at the time; however having subsequently reflected on this aspect of their pregnancy experience, many women expressed concerns over the extent to which they had been in a position to make a meaningful and deliberate choice. For some, such concerns related specifically to a perception that they had been given an inadequate amount of information on which to base their decisions (see later discussion). Other participants framed their concern in terms of 'misrepresentation' and even dishonesty in the provision of their prenatal care. A sense that a serious issue was being handled with inappropriate levity was a common theme, something which Naomi referred to as "a heavy thing treated as a light thing":

I sort of felt like I was suddenly thrown into this world of having to make a serious decision...that dawning that this was a serious test, not a test because it would be nice to see a little baby sitting in there.

Similarly, Rona indicated that the manner in which screening had been presented to her did not acknowledge what she regarded as the existential significance of questions raised:

I think it's being presented as kind of like "would you like an enamel finish on that, or stainless steel finish on that?" and actually it's "where do you think that soul came from? What do you think happens after death?" And it's all those issues. And I think it's being presented as a really simple issue, like "do you want a disabled child, or would you rather have a child who's not disabled?" and it's not like that.

While most participants did not recount an explicit sense of compulsion to undergo screening, for some the casualness with which it had been presented appeared to 
contribute to the logic of reassurance attached to the general purpose of antenatal care.

Eva:

Yeah, I mean it was presented to me as though that was just "this is what you do, now you're pregnant this is what you do", and it wasn't like something I could have or should have said no to, funnily.

In a number of cases, the primary purpose of an ultrasound scan had been explained as the confirmation of the pregnancy itself or estimation of gestation, rather than for detection of abnormality, as in Naomi's experience:

Oh well it was, you know, (I) found a midwife, went along, she said oh you know you can have a ...the 12 week scan, should book in for that and oh you know it'll give you your dates, make sure you've got accurate dates. And I sort of thought oh yeah that sounds alright, I didn't know anything about whether scans are good or bad, or whether there's any reason not to have them. And I just felt that it would be a good idea to just see that the baby was real and was there, you know, to feel pregnant.

Similarly, Kerry stated that she hadn't been informed of the purpose of the scan, noting: "It was just sort of seeing that it was there really".

In contrast, within Ruby's account the purpose of the scan was explicitly stated, however she felt the significance of the test was downplayed. As she recounted the words of her caregiver: "Do you want us to do the tests for Down syndrome? They're just the standard tests."

Sandra did not recall receiving any information about the purpose and potential outcomes of a scan, a fact she subsequently identified as leaving her totally illprepared mentally and emotionally for the discovery of a major abnormality:

The only thing (he) told us is prepare yourself by drinking lots of water, um, (we) went and had the scan and at no point...I never got the impression that there was any choice about having it or not, just completely routine and at no 
point was there...I certainly didn't think about um whether everything would be ok, and um, yes, it was just sort of a standard kind of thing, that this was what you did, and I just didn't even consider that there would be any issues...

A number of women expressed the concern that clinicians had not clearly articulated the link between diagnosis of abnormality and termination of pregnancy as a 'default' clinical pathway. Sometimes this omission appeared to be interpreted in terms of deliberate misrepresentation. For example, as Petra comments:

I think it's dishonest, because they know why they're screening you, but you're not being advised of what you might have to face with that screening. You go in "oh I'm going to see my baby", I don't know anybody who's had a different idea from mine "oh I'm going to see my baby" when they went in to an ultrasound, it was just ok...well, (it) felt silly. This is just silly, this is just toy stuff but hey I'm going to see my baby and isn't that nice? And I don't know of anybody who was aware that the professionals were going to be looking for things wrong. (It's) in retrospect quite dishonest. And unpreparing.

Rona's comments show a similar view of screening as having been 'dishonestly' represented, and suggest that more broadly she views the term 'screening' itself as implicitly misleading:

Because that's ultimately what it comes down to, I mean you're screening, but it's not like you're screening and saying "oh you know during your pregnancy you need to have an orange every day". That's not what's happening. It's you're screening, and then you're deciding whether to terminate the pregnancy.

Rona's evaluation resonates with Press \& Browner's (1994) contention that both pregnant women and clinicians attempt to dilute the moral discomfort around screening by participating in "collective fictions" around the purpose of screening, whereby this practice comes to be framed by both parties primarily in terms of therapeutic monitoring rather than diagnosis per se. As they suggest, this allows a conceptual distancing of prenatal screening from the practical contingencies of selective termination. The presentation of screening as routine, they argue, establishes 
a sense of inevitability which dilutes the burden of reflection, making it possible to "leave open but uncontemplated the option of terminating an affected pregnancy" (1994:203).

\section{The experience of 'risk'}

With the increasing emphasis in contemporary public health policy on the value of population-based rather than risk-based screening programmes ${ }^{81}$, a growing number of pregnant women will be confronted by the psychosocial consequences of being designated 'at risk' (Heyman, et al, 2006). Irrespective of an individual's evaluation of their own experience, a diagnosis of risk draws them into a schema of intervention and management, insistently marking their experience as pathological; as Lupton suggests, "To be designated 'at high risk' compared with others is to be singled out as requiring expert advice, surveillance and regulation." (1999:61).

Of those having undergone prenatal screening, four participants had received a clinical designation of being at 'high risk' of fetal abnormality during a pregnancy on the basis of screening results. Subsequent to this news, each of these women was then either offered further screening to clarify the diagnosis, or the option to undergo a termination of pregnancy. Within these women's accounts, key themes of concern related to a sense of a lack of adequate information and psycho-social support in coming to terms with an abnormal diagnosis. After receiving a positive diagnosis, ${ }^{82}$ the sense of shock and uncertainty was often compounded by the long delay

\footnotetext{
81 This broad shift in emphasis within the contemporary public health domain is discussed in chapter five.

${ }^{82}$ I.e. a result which is either strongly suggestive or definitely diagnostic of the presence of fetal abnormality
} 
frequently associated with clinical confirmation of initial screening results through follow-up tests. ${ }^{83}$

In Ruby's case, while amniocentesis was recommended, the pregnancy was not yet sufficiently advanced to perform the test, and she describes the impact of waiting for a period of three weeks prior to undergoing the amniocentesis, and three weeks after until the result was confirmed as negative:

I waited... and got more and more stressed about this amnio, because yeah the things which kept going around in my mind were this nurse saying you know there's a really high chance of things going wrong, and the doctor saying I should have terminated, and I was thinking "oh my god I'm going to have an amnio and then I'm going to find out this child has got Down's and it's got cardiac problems, and it's probably going to die in utero because that's apparently what is likely to happen". So I got, I turned myself into a little stress-muffin.

An unusually long (slightly over three weeks') wait for the result heightened Ruby's sense of anxiety as she began to doubt whether, if the result was positive, the pregnancy would have now passed the point at which a termination would be permitted:

...after three weeks you know I was thinking "Oh my god". Because I was considering having a termination if it wasn't alright....and I was just panicking by that stage I think.

Ruby's chief concern about her experience related to what she saw as an endemic insufficiency of information with which to evaluate the best course of action for herself:

Yeah nobody ever spoke to me. I think what I found hardest was there was just no information for me to base these decisions on. Like when I spoke to the obstetrician, I felt like I was making a decision in a vacuum, I was expected to make these really important decisions without actually being aware of, you

\footnotetext{
${ }^{83}$ Typically through amniocentesis, a genetic-based test which requires the culturing of fetal cells in the laboratory. The result takes on average two weeks to be confirmed.
} 
know, what the consequences were, what things really meant...I didn't have anyone to turn to, and so I was sort of negotiating my own way through the hospital system... Also what this "one in twelve" or whatever chance it was, what it meant...I wanted something so that I could make some sort of juggle, whereas I just felt it was a totally random decision that I was making.

Where information had been provided, such as the risk estimate for Down syndrome based on the NT scan, she indicated that this had not been conveyed in a way which she was readily able to interpret.

For Anna, the designation of risk was an unwelcome status, and one which she describes as having overshadowed the duration of her entire pregnancy. Enlarged kidneys detected during an ultrasound scan suggested the possibility of a severe form of infantile kidney disease, a prognosis conveyed to her as "pretty grim news". The pregnancy was overseen by a 'high-risk' midwifery and obstetric team, and Anna underwent close monitoring through ultrasound for the rest of the pregnancy, a process she describes as continually reigniting her sense of anxiety

The week before we were having our ultrasounds we'd psyche up, and going into (hospital name) was absolute terror. And we'd think "oh, what are we going to hear now, you know?" And so then afterwards we'd be really depressed for a week, and then we'd be alright for another two weeks, and then we'd have to go in again.

While Anna expressed deep ambivalence about the value of this intensive monitoring, she described a sense of being unable to disentangle herself from the process of medical management set in motion by the initial diagnosis.

I felt we were caught in this trap, you know? I couldn't get off it. We were in the system and we couldn't get off it. And then I think even my GP, midwife thought it was over the top. I did think at one stage "when could we stop, you know, can we stop this? " 


\section{Insufficiency of information}

Such themes of loss of control and de-personalization, of being 'in the system', are reflected in a number of participants' accounts of screening, and the inadequate provision of information seems to be perceived as significantly contributing to this sense of powerlessness. Interestingly, several women used the metaphor of a conveyor belt to characterise this experience. For Sandra, the sense of a lack of personal autonomy in the context of her care results from both a medical and societal expectation that it is not necessary for women to actively evaluate screening:

I think there should be some kind of information that says "these are some of the problems that can be found and some of the choices that you might have to think about", and sort of have that time to think about whether you want to be screened. I just...you're just sort of on a conveyor-belt, (it's like) "yeah yeah, pregnant, you go to the doctor, oh yes it's time for the screening", you know. And everybody else does it, then you just go along with it.

Patricia similarly draws on a mechanistic metaphor and describes the struggle to make a decision in the context of a lack of information and lack of professional attentiveness:

Everyone was offered it, (I had) no idea why I was having it, and then when I was given the statistical result from that (it) wasn't clear to me what that meant, but I was then on the track, and the next thing was well then if you get this result you have an amnio. And I think probably at an earlier stage it would have been nice to have somebody sit down and say this is what it all means, and nobody did that. And then it felt like very suddenly we were faced with a decision about terminating.

For some women, a lack of information at the point of a positive diagnosis had material consequences which they later came to view in terms of having been deprived of opportunities to make alternative choices, or at least to fully consider a range of possible experiential trajectories. A number of women expressed a concern 
that insufficiency of information had left them vulnerable to directive counselling on the part of their caregiver.

Following a diagnosis of anencephaly, ${ }^{84}$ Sandra felt a clear sense that the expected and appropriate course of action was to terminate the pregnancy. There was no discussion of the pragmatic or emotional implications of allowing the pregnancy to continue to term, or provision of information on support available for those wishing to make this choice:

And at the time he also said the options are termination or keeping the...you know, taking it through to term, but um, got a pretty clear impression that the best thing to do would be to terminate... I always remember the doctor saying "it's not compatible with life", that particular defect, that um there was very little choice - it wasn't like spina bifida or Down syndrome where you might think, "god, do I or don't I?" . It was (like) "I don't really see that I have a lot of choice apart from termination because the outcome is not going to be a, you know a baby that's going to live", and in a lot of ways I think that that might have made it easier to go through with that decision.

Sandra's comments suggest that while she felt that the doctor's directive approach may have helped her to make a difficult decision by framing the choice as clear-cut, she was nonetheless denied an opportunity to 'make sense' of this traumatic experience on her own terms. As she notes, in subsequent years, after researching the experience of fetal loss she realized she hadn't been made aware of the existence of support groups for women deciding to continue abnormal pregnancies, and the options which might be available in terms of processing the death of a baby after birth, including the practice of donating organs to live infants. That she had not been in a position to explore these options at the time seemed for Sandra a lingering regret in her narrative of this experience.

\footnotetext{
${ }^{84}$ A lethal genetic abnormality of the fetus characterised by the malformation or absence of the brain.
} 
Similarly, Patricia had concerns about the long-term implications of decisions made in the context of incomplete information, feeling that the already difficult decision to undergo a termination consequently held the potential for the agony of future doubts:

I think that you know for some people years on the trauma endures, and you know the tragedy of it, they never get over it. And that's exacerbated if at the time of making the decision, or having the baby or not having the baby, you're denied critical bits of information so that you revisit it years later and say “fuck, if only I'd known that, or if only they' $d$ told me this, or why didn't they..." If those things are left unresolved, then the trauma I think is much more serious... when I think back to our experience, it might have been, it would have been nice if the doctor had said "well look let's just talk about the consequences or the implications or what this might mean, and maybe you want to give this some thought..." But that didn't happen, and so it was a very perfunctory five minute consultation, and we walk in and we're having a baby, and we walk out and we're not.

Patricia describes an implicit sense of medical directiveness following a genetic diagnosis of Down syndrome, and, similar to Sandra's account, a lack of alternatives to termination:

One thing that I felt, in fact it was expressed by the doctor who gave us the news of our baby's Downs syndrome, it was almost like he said, I don't know what he said, but it felt like he was going "right decision, yes", you know, big tick, because he said something like "I think that's very wise", oh I cant remember what he said, and he might have equally said something affirming if we'd said the opposite, but there was a ... I got the sense we were, in terms of the medical profession's view or his view, that we were doing the right thing because we hadn't chosen to have a baby with a severe abnormality, you know..."boy that's a drain on the resources of the health sector", you know. So I got the sense, now whether I might have imagined it, but, whether every time you have a screening technique that's potentially an expensive one, and certainly this technology that's behind it's expensive, whether with some medical professionals, it's not said but it's implied, that "look, we've gone to all this trouble to find out that your baby has this. Do the right thing".

Alosina similarly felt that the manner in which screening had been presented to her expressed an assumption that termination was the logical outcome of positive diagnosis. In her account this was experienced less as a subtle pressure than as explicitly directive: 
I think I was perfunctorily told, you know, what the purpose of the test was. It was only after the test results came back that the consequences were outlined in graphic detail, you know? And the consequences that were put to me were "It's going to ruin your life, and you should terminate".

Like a number of other participants, Alosina expressed concern at the lack of discussion about alternatives to termination, and in particular the possibility of continuing a pregnancy after diagnosis of Down syndrome, and what such a decision might entail:

And there was no like "Down syndrome does this" ... and this is what I didn't like, there was no kind of acknowledgement that Down syndrome has a spectrum. It was all "Down syndrome? It's the end of the world". And I didn't appreciate that coming at the ... after the test results had come in. I thought that Down syndrome was really almost apologized (for), like you know, it was like "these people are dysfunctional and cannot function in this world", rather than saying "well, there are ranges of Down syndrome". That was never presented to me, you know?

For some participants, the designation of 'risk' which attended the information produced by screening was regarded as having deprived them of an experience which had been pleasurably anticipated as a positive life experience. In Anna's case, a diagnosis of abnormality did not in itself undermine her own sense of her pregnancy as something positive and 'healthy', however, as she suggests, the insistent medical framing of her pregnancy as 'risky' undermined her own positive view, leaving her in the longer term with a lingering sense of disappointment:

I felt cheated, and I feel cheated now... I feel quite angry towards the medical profession actually, that they took that away from me. Because I felt like I was carrying a healthy baby with (child's name), I did not feel it was a sick child. And I found that very conflicting ... so you'd go along and be told these awful things, and (be) told that there was a high chance he had infantile (kidney disease), and I couldn't believe it, because I felt so well, you know?

Such accounts highlight the way that what counts as 'useful' information may vary between medical and lay contexts, and raises questions about what appears to be a core presumption within contemporary biomedicine; that is, that the value of 
screening technologies necessarily lies in the pursuit of the maximum clinical data possible. As noted below, concerns around this issue emerged as a separate theme.

\section{"The right not to know"}

While a lack of information about the rationale, procedures, and possible outcomes involved in prenatal screening is a theme common to many accounts, this concern relates overwhelmingly to matters of individual choice and consent, and the extent to which a considered and informed choice was able to be made. Running parallel to this is a questioning of the value of information obtained through screening procedures, and specifically, the personal value and practical utility of this clinical data. As Press \& Browner (1997) note, pregnant women typically cite 'information' as a chief benefit of screening, however they suggest that this in no small part reflects broader cultural norms, and in particular, the entwinement of consumerist and scientific discourses which express the imperative to obtain maximum possible information.

Nonetheless, a critique of the assumption that medical information is valuable per se was common to a number of accounts. As Melissa noted:

When you first get pregnant and you've got so much information coming at you, and there is that sort of just temptation that the more you know about the baby the better ... I mean I know I had it in my first pregnancy particularly, that "if I know more it must be better, the more I can find out and I can know that my baby is ok, the better it is". And it was only later when I thought well it doesn't quite work like that.

Naomi stated that the opportunity to critique what she regarded as a contemporary

fetish for 'data gathering' had been a motivation for her participation in the study:

I guess I was motivated by what I saw as the increasing amount of ability to get tests done, and the sort of culture of just getting them done, you know, doing the test, doing the scan, finding out all of this information. When actually sometimes it's better not to know. And it's a really hard thing to actually suggest that you may not want to know. I think everything in the health system points you towards informed consent, but doing all these things. And that maybe it's worth also being offered the option of not finding out. 
Within her own experience of declining prenatal screening, she resented the fact that in spite of the rhetoric of informed choice, the option to take a position of what she regarded as informed ignorance, something she described as "the right not to know", was neither medically nor socially sanctioned. ${ }^{85}$

A number of women conceived of the option of informed ignorance less in terms of a 'right' or a matter of informed consent, than as a welcome exit from the negative spectre of risk, and the difficult choices raised by screening. Eva noted that she would welcome the opportunity to experience an 'uninformed' pregnancy by declining screening in future pregnancies: "I'd just go into it blindly really now. I think ignorance is great actually". Anna commented that she had come to regret undergoing screening: "I wished I'd been like my mother, and just discovered it (i.e. at birth), and just got on with it". As a more profound example of the choice of 'ignorance', Rona's decision not to undergo any form of screening will be discussed in the following chapter.

In Kiri's case, screening had failed to detect Down syndrome, and she had only learned of her baby's condition at birth. She views not having this knowledge during the pregnancy as having enabled her to experience a pregnancy which was not evaluated in terms of risk or abnormality:

I personally feel that that was a really good thing for me, because society being as it is, it would have been a negative experience for me during pregnancy. So I would have been told about all the negative aspects of the condition, and I would have seen him as a condition instead of a person, before even giving birth. So the bonding experience wouldn't have been there. So I'm really happy that I didn't know.

\footnotetext{
${ }^{85}$ It is interesting to consider how such examples of lay evaluations of a right or benefit associated with 'not knowing' may also be at play in other areas of health screening, in particular those characterized by comparatively low rates of patient uptake, such as screening for prostate cancer.
} 
Eva similarly framed a lack of clinical information as having been personally

beneficial in coming to terms with her experience. In her case, a lethal congenital abnormality had not been detected during a routine ultrasound scan. The baby died several days after birth. In her analysis, the failure to detect the abnormality is viewed less as a medical failure than as a 'happy accident' which enabled her to ultimately accept the baby's death on her own terms: "I was happier having gone through the pregnancy, had the baby, met my child, buried our child, than some authority coming in and saying "(do) this this this".

Such perspectives are radically at odds with popular notions of appropriate maternity and in particular the view that 'being informed' is a fundamental responsibility associated with pregnancy (Anleu 1997, Press \& Browner 1996). However, as Rona's account suggests, alternative notions of maternal responsibility exist which constitute engagement with the imperatives of information and risk as in itself a form of risk, and an expression of an unsatisfactory degree of maternal acceptance:

I got annoyed when someone suggested I was being irresponsible by not screening. And that annoyed me because I thought actually, no, what I'm doing is making a decision that is actually really accepting and really responsible, as I see it. And is not putting anybody's health at risk, and quite the reverse.

In Rona's assessment, engaging with prenatal screening reflects an unaccepting attitude which constitutes a potential harm to a fetus, with potential consequences in terms of parent-child relationships:

I think when you're screening a baby what you're saying is you're saying are you good enough to carry to term, or should I kill you. And I think that that is not a very nice message for the baby to receive in the womb. I think you want to be sending the message to the baby, or I want to be sending the message to the baby, or I think what would make for an easier parent-child relationship is "I love and accept who you are". 


\section{Acceptance and surrender}

Alongside a questioning of the inherent value of clinical information, a motif of acceptance, such as that reflected in Kiri's account above, emerged as an additional counter-theme to mainstream discourses of choice and risk. For example, Eva regarded the framing of screening in terms of 'choice' as symptomatic of an unrealistic cultural obsession with control:

I think because there's no guarantees in life, and I think that's part of our thinking. I think it's like this designer thing and everything's going to be just A-OK. We can't have that thinking because actually life's not perfect, life can't be perfect and don't let any authority make us think that everything can be fixed because it actually can't. That's reality, you know, you start to get out of touch with reality if society's going to say "designer baby - oh you want a girl? Yep fine have a girl. You want it to be disease free? Yep, fine", you know. But actually life goes on, and even after that baby's born something might happen. Yeah, so we fool ourselves if we think we can have control.

Similarly, Petra expressed a concern that the notion of 'choice' gave women an illusory sense of control, leaving pregnant women (and in particular first time mothers) unprepared for the possibility of unexpected outcomes, and without the resources to 'move through' a negative experience:

I think that when it's new to you, you think there's a whole lot of stuff you can control, and if you just do this, then you know that won't happen and things will be normal. But it's not like that, life isn't. It just seems life is what happens ... if you think you've got things under control then you haven't, 'cause you have a bad reaction when the things that aren't controllable happen, and because you thought you had it under control, you know?

Within these and similar accounts, participants' draw on notions of fate and destiny in accounting for their rejection of ideologies of risk and control. As Marama stated, "I sort of felt destiny should take its course". Naomi expressed a similar view in the remark "it's a time that you sort of need to take what's given to you". And Kiri stated 
I probably believe in what's meant to be. I really do, and I feel that if the baby is meant to be born I think it should be, it should be born and then if it needs to pass on it needs to pass on. So I feel one hundred percent that it needs to be born. And then you just let nature take its course from there.

As a counter-theme to the discourses of choice and risk which characterise mainstream screening discourse, such motifs of acceptance and surrender constituted a surprisingly common theme in participants' accounts. Participants' use of these and similar metaphors in accounting for their decision-making will be discussed further in the following chapter.

It is significant to note that a number of women who had previously undergone screening indicated that they would be unlikely to accept it in future pregnancies; this included two participants who had undergone terminations following diagnosis of fetal abnormality. Such findings substantively problematise the equation of the availability of screening with greater individual choice, and suggest that choice per se ought not to be neutrally evaluated as universally positive.

\section{Disembodiment and provisionality}

Lupton (1999) argues that the discursive construction of pregnancy as an inherently risky domain draws every pregnancy into a status of provisionality. Within the context of selective screening, the evaluation of an individual's 'genetic risk' compounds this sense of tentativeness in requiring women themselves to consider whether a pregnancy should or should not proceed (Rothman 1986, Rapp 1987). Whether or not they had personally experienced a diagnosis of risk, participants variously indicated that their engagement with prenatal screening had generated a sense of ambivalence in their commitment to their pregnancies and in their psychological and emotional attachment to their fetuses. As Melissa commented, "I 
think it keeps that whole pregnancy provisional for longer before you can really sort of enjoy it, and wallow in it, and think about the baby and things".

Petra's account suggested that her experience of amniocentesis had altered her relationship to the pregnancy by generating a sense of temporary provisionality. As she recalled: "When I had the amnio, I was very aware that it may not be a pregnancy I continued". As she explained, this sense of detachment subsequently resolved after she received a diagnostic 'all-clear'.

For those participants who had been designated 'high risk' by dint of an abnormal fetal diagnosis, this sense of provisionality took on another dimension of significance and was responded to in a number of different ways. For Ruby, a six-week wait between an incorrect initial diagnosis of Down syndrome and confirmation of a normal result was an experience which she managed through a strategy of deliberate emotional detachment. This was in part achieved by focusing on potential 'worst-case scenarios', such as the prospect of losing the baby either though miscarriage or an eventual decision to terminate: "The whole period I was mentally prepared that I was going to lose the baby, so I was trying not to be, I was trying to be very detached from the whole thing".

Ruby tenaciously maintained this strategy of detachment, even when, during a followup scan after her initial abnormal diagnosis she was confronted by the (inappropriate, given the circumstances) comments of medical staff which seemed intended to encourage her to form an emotional connection with the on-screen fetus: "They were saying 'oh look at its little hands', and I was thinking 'yeah, so these little hands are going to die really soon, aren't they.", 
The impact of this self-imposed period of emotional 'suspended animation' persisted until the birth of her baby, a moment which she describes in terms of finally being able to materially 're-embody' her experience:

I didn't ever think the word 'baby', I talked 'fetus', because you know, that's a thing. And even during the labour ... the midwife asked me what sort of nappies I'd brought in or wanted to use, disposable or cloth nappies, and I sort of went "oh my god, that's right, there's a baby in there". 86

As Heyman et al (2006) and Green \& Statham (1996) have noted, the impact of a 'high-risk' status sometimes has long-term psychological consequences for individual women, including residual anxiety which may persist even after a designation of risk is no longer relevant. This appeared to be true of Sandra's experience. Subsequent to her experience of a positive diagnosis of anencephaly and the termination of that pregnancy, Sandra went on to have another child. Even though this pregnancy was clinically 'normal', she describes the experience in terms of something to "get through", characterising the pregnancy as "nine months of worry". The following comment suggests a sense of bodily disengagement, and a relinquishing of the possibility that the pregnancy might be a pleasurable experience:

I guess it was more just the body's the vessel and "let's get on with it" kind of thing. And possibly it is as a result of the first pregnancy of feeling like that the second time, and thinking well I just want to get through this rather than maybe really really enjoying it and all that sort of thing.

Anna describes a sense of disembodiment which results from her clinical management as an 'at-risk' patient. In particular, she describes feeling as if she was regarded simply as the physical container for her unborn child was in fact the primary focus of medical interest. While rejecting her own 'high-risk' designation - 'I just didn’t take it on" - she nonetheless felt that any attempts she made to normalise her experience were invariably undermined by the medical paradigm she had been positioned within

${ }^{86}$ See chapter five for further discussion of Ruby's account. 
since the diagnosis of abnormality, and that her own values and preferences drew little consideration. This sense of disembodiment also seemed to be experienced as the result of a tension between her needs, and those ascribed by clinicians to her fetus. As, in her understanding, the genetic condition did not require immediate intervention after birth, Anna somewhat resented this apparent privileging of fetal interests over her own wish to give birth at a familiar, low risk hospital. ${ }^{87}$ Once her pregnancy had been designated 'high-risk', this was a preference she felt she had to "fight for", in spite of the fact that her own midwife and general practitioner were willing to attend the birth in a 'low-risk' setting:

I just think even with my background of wanting to have a normal delivery I had to really fight for that, and you know, really persuade them that...I just think it wasn't taken into consideration, My mental state wasn't taken into deliberation, that I would want a normal delivery with people that I knew around me. They were just thinking of this child. And they probably wanted it in (name of tertiary hospital), I mean I felt like it was obviously an interesting case ... They weren't looking at the whole thing as a family dynamics.

For Alosina, a diagnosis of risk drew an entirely different response, sparking a sense of defiance and protectiveness towards the pregnancy. Following a NT result strongly suggestive of Down syndrome, Alosina describes facing considerable pressure from her partner (in addition to the sense of medical pressure described earlier) to undergo amniocentesis in order to confirm the result. While she ultimately agreed to have the test, her reluctance to engage with assessments of risk and provisionality appeared to consolidate a sense of commitment to the pregnancy which transcended any concerns around the social or practical implications of disability:

\footnotetext{
${ }^{87}$ Anna had been advised to give birth in what is known in New Zealand as a 'tertiary' level hospital (major hospitals which service 'high risk' referrals from centres less well equipped to deal with these cases). Birth in the 'tertiary' hospital, rather than a nearby regional hospital, would have meant that her care was handed over by her own midwife and GP team to a specialist obstetric/pediatric team.
} 
I think that yeah it made me value and treasure the pregnancy much more. I was fierce in my defence of that fetus, I was like "No one's going to ... dah dah dah", you know? And maybe without that test I wouldn't have been so protective. It was quite intense, like I was so determined to have the child, and I was envisioning my life as a solo parent AGAIN (laughs) you know? With two children now, and one Down syndrome. And I was saying to my son, I was like "Ok if you see a Down syndrome child, that's our future, and we have to appreciate a whole range of capabilities and stuff... "I mean I was being dramatic in some ways, but I just couldn't believe the attitudes that I was kind of confronting, you know?... I was just like "I will fight, you know, I will go tooth and nail for this one".

Anna's account similarly suggests a rejection of risk as the only possible frame of reference for evaluating her pregnancy. Her experience highlights the disparity between the medical evaluation of her child's prognosis, and her own assessment. After birth, her child was discovered to have additional digits, ${ }^{88}$ something which was not detected during multiple scans throughout her pregnancy. The obstetrician who had conducted the scan told her he was "gutted" he had not detected these during the pregnancy. In contrast, Anna explained that she was in fact deeply relieved, as she felt she had avoided extra pressure to end the pregnancy:

And he was quite gutted that he hadn't got it right. And we said to be honest we were really thrilled that you hadn't because it would have ... and he never said anything because he realized that, yeah, we'd have probably been offered a termination then and there.

In spite of the grim prognosis given during her pregnancy, Anna's evaluation of her son's progress since birth is that he is a well child. Her comments suggest that some women do not necessarily equate a genetic abnormality with ill health:

And so he's being monitored by every Tom, Dick and Harry - seven months (old) and he's fighting fit, if you look at him, he looks really good. But he's, yeah, they don't necessarily know what's wrong with his kidneys, but he's doing a lot better than we ever could have imagined.

The themes which emerged in these accounts suggest that the moral bases of women's screening decisions do not necessarily reflect the prevalent cultural norms embedded

\footnotetext{
${ }^{88}$ Extra digits (i.e. fingers or toes) are often, but not always, clinically associated with chromosomal abnormalities.
} 
in the mainstream 'story' of prenatal screening. The following chapter will now explore the moral framework of participants' decision-making. 


\section{Chapter Four Screening decisions as 'moral work'}

As the previous discussion suggests, whether or not a decision is made to undergo prenatal screening, for many women this choice is marked by a generalised moral ambivalence. It is clear that such decisions do not necessarily equate with wholesale acceptance of the necessity or value of this practice as a norm of pregnancy care. Accounts such as Anna's signal the effort involved in 'meeting' the various tensions attached to screening, suggesting that for some women considerable moral 'work' is required in deciding whether or not screening will be accepted, and, more specifically, what the terms of this engagement will be.

This section of the discussion considers the specifics of the hidden moral work pregnant women undertake in their decision-making, by examining some of the ways such decisions are reached and accounted for within participants' accounts. As noted in chapter one, Raynor Rapp was among the first to theorise this work in her characterisation of pregnant women as 'moral philosophers of the private' (1999a:306), ${ }^{89}$ framing women's prenatal decision-making in terms of the "working out of an experiential trajectory through which profound existential dilemmas could best be understood and internalized" (1998:59). While the term 'moral work' is increasingly used in contemporary health-oriented research (and in particular, that which examines questions relating to the ethics of health care practices) this notion is

\footnotetext{
${ }^{89}$ This influential trope has been revisited recently by Williams et al (2005) in their analysis of recent innovations in prenatal screening technologies. This research, and recent investigations of lay health decision-making (Scully et al, 2004, 2006a, 2006b and 2007) was especially important in my decision to position one of the aims of this thesis in terms of an investigation of 'moral work'.
} 
often not clearly defined or attributed to any particular source. ${ }^{90}$ For the purposes of this thesis, my understanding of moral work follows Frank's $(1995,1997)$ exploration of the work undertaken by individuals facing difficult or personally 'high stakes' experiences (such as living with chronic illness). His analysis essentially defines this work as those various internal processes (including the reconfiguring of 'official' accounts of illness into personal stories) undertaken in the attempt to make such experiences meaningful and personally acceptable, consistent with one's personal 'moral world'. In line with recent qualitative analyses of lay health decisions, I have taken such work to include processes of deliberation and decision-making.

In theorizing this work, I have also been broadly influenced by feminist science studies theorist Charis Thompson's (previously Cussins) notion of 'ontological choreography' (Cussins, 1998). Thompson defines ontological choreography as a process by which individuals engaging with medical technologies (and specifically, reproductive technologies) necessarily organise or choreograph a "functional zone of compatibility" between ontologically distinct 'things' in responding to this experience (Cussins, 1998:192). In this conception such things are seen to include both material entities (such as the physical body, and medical and technological equipment) as well the array of norms (medical, socio-cultural) attached to the encounter for the woman who as a patient/client, and as infertile/potentially fertile, occupies contradictory subjectivities of compliance and autonomy, failure and success. This process of choreography is necessary, she argues, in order to preserve a stable sense of a 'longrange self' in the midst of a potentially alienating or disembodying experience (she cites the highly regimented and technical character of contemporary fertility treatment

\footnotetext{
${ }^{90}$ In particular, such usage is evident in contemporary nursing research. See for example, Tiedje (2000) and Storch \& Kenny (2007).
} 
as an example of such an experience). Thompson's work focuses specifically on fertility rather than maternity care per se, ${ }^{91}$ and working as she does within an 'actornetwork' model of agency, her analysis weighs the significance of structural determinants of individual agency somewhat differently than does this research. However, extracted from this particular context, this notion of choreography is useful in considering women's engagement with prenatal screening, not least because it captures the sense that active 'work' is involved in navigating and indeed managing the various dimensions of this body-oriented experience. Furthermore, it seems to me that a conception of the multiple norms circulating in mainstream screening discourse as ontologically distinct is useful in signalling the existential character of the response required to make sense of such tensions.

Rapp (1998) observed that women engage selectively with prenatal screening, utilizing some technologies while refusing others, noting the significance of nonscientific constructions of pregnancy and motherhood in women's refusal; ${ }^{92}$ however she did not elaborate in depth on the specifics of this. Indeed there has been minimal subsequent social scientific research exploring the reasons for such refusals by pregnant women. ${ }^{93}$ The following discussion considers some specific examples of resistance to the norms of prenatal screening in participants' accounts, considering the extent to which women can be seen to negotiate the terms of their engagement with

\footnotetext{
${ }^{91}$ See also Thompson, (2005).

${ }^{92}$ Campbell (2008) notes that the selective uptake of screening is also well recognized within midwifery practice, where it is regarded as a right of informed choice. See discussion of late booking for pregnancy care later in the chapter.

${ }^{93}$ More recently, however, sociological interest in lay attitudes towards genetic screening per se has begun to increase, including an interest in the critique or refusal of screening for inherited conditions by adult individuals (see for example, Callon et al, 2004, and Scully et al, 2004, 2007).
} 
screening. The discussion then considers the moral framework of participants who chose not to undergo screening.

\section{'Trading off' unwelcome aspects of experience}

Charmaz (1995) has argued that when facing disruptions of a stable sense of self (such as during the experience of chronic illness) individuals often attempt to negotiate 'identity trade-offs'. This conception suggests a negotiative or selective engagement with medical intervention, whereby unwelcome aspects of a new identity (i.e. as a 'patient') may be tolerated only if other projects of identity are preserved. This resonated with the accounts of several participants. For example, Anna's comments suggested that she was only prepared to accommodate the clinical imperatives of 'high-risk' pregnancy care if she was able to 'set the agenda' in terms of her birth care. As noted in the previous chapter, this involved a preference for a 'low-risk' setting in a provincial hospital with familiar caregivers, rather than a tertiary-level (i.e. 'high risk') centre in a main city:

I didn't want to deliver in town, because I didn't want ... I would be offered an on-call obstetrician and whoever-I-get-on-the-day midwife, when I had a GP and really good midwife here. So I said no I didn't want that. So I had to fight quite strongly to deliver in (name of provincial hospital).

Anna's account clearly indicates a negotiative engagement with the machinery of prenatal care; as she states; "because it had been such an awful pregnancy, I wanted to have a normal birth experience, you know, relatively normal birth experience, whatever happened". In choosing the terms of her birth, she attempts to retrench the sense of personal control she feels she has lost amidst the categorisation of her experience as 'high risk'. 
Wilma's engagement with screening is similarly negotiative in character, and reflects a selective approach to the norms of prenatal care whereby screening is framed exclusively as a health check rather than a diagnostic event. In doing so, she effectively deflects the 'high-risk' designation usually applied to women in her age bracket. ${ }^{94}$ Whereas Wilma states that she values the ultrasound scan, she appears to frame this only in terms of an opportunity to 'check' the health of her unborn and simply does not engage with notions of genetic risk. Although prepared to undergo ultrasound on this basis, Wilma refused to undergo amniocentesis, which she recognized as a diagnostic (and therefore by definition unwanted) procedure: "It was kind of an unwelcome intrusion, but I was quite firm in what I wanted". It is clear that according to Wilma's moral schema, the decision to refuse a medical recommendation to undergo amniocentesis nonetheless fits within a conception of good and responsible mothering. While she held a positive view of medical technology in general, her 'acceptance' of scans in no way reflected a broader endorsement of prenatal screening as a diagnostic project. In spite of the designation of her pregnancy as 'high-risk', as in Anna's case, Wilma insisted on giving birth on her own terms at a local 'low risk' maternity unit, rather than in a major hospital as her caregiver preferred.

Alosina's account further illustrates the attempt to 'dilute' the unwelcome impact of a 'high-risk' designation, and shows the way that family members, and not only clinicians, may ascribe a schema of risk to women's experience. Following a scan which suggested a high likelihood of Down syndrome, Alosina described how she reluctantly agreed to have an amniocentesis in accordance with her partner's wishes:

\footnotetext{
${ }^{94}$ Wilma was aged 44 during this pregnancy. Obstetric literature designates pregnant women over the age of 35 as within a 'high-risk' category.
} 
I didn't really want to have the amniocentesis, and I didn't like the risks, and you know, I was very resentful but I took the risk and had the amniocentesis. And I did all the research on the side about Down syndrome, and coping with Down syndrome and how we can help people with Down syndrome have what we call normal lives, and I was almost determined that I was going to have the child no matter what, and it kind of put my relationship in a crisis because we had such diametrically opposed opinions on termination in this case.

In researching Down syndrome 'on the side' and resolving to continue the pregnancy irrespective of the test outcome, Alosina appeared to be seeking to balance her partner's ascription of risk to the pregnancy with her own more positive evaluation. As she stated "I think that yeah, it made me value and treasure the pregnancy much more. I was fierce in my defence of that fetus".

Variously describing selective levels of engagement with prenatal screening, these accounts collectively speak of an attempt to retrench a sense of agency sufficient to 'trade off' the unwelcome aspects of experience. In doing so, it seemed that these women felt they had been able, in lesser or greater degrees, to reassert personally acceptable (and distinctly 'non- mainstream') frames of reference for their decisionmaking.

\section{Embodied knowledge as a moral resource}

As mentioned in chapter three, a number of women questioned the personal utility of scientific data produced by screening, instead emphasizing the significance of their own past experience and sense of physical wellbeing in guiding their decisionmaking. These accounts indicated that these forms of 'embodied' or intuitive knowledge were conceived of as an authoritative alternative to biomedical understandings of pregnancy, and were often utilized to account for the rejection of a 
risk-oriented model of pregnancy care. ${ }^{95}$ For example, several women were highly dismissive of the equation of older motherhood with a 'high-risk' pregnancy status. Wilma indicated that in her view, her age and past experience made her more rather than less equipped than younger women to carry out a healthy pregnancy:

I would have been 41 for (child's name) and 44 when I carried (child's name). Which to me it wasn't really old, and I just felt that I was fitter and more able to carry a baby than some of the younger women anyway. So to me the age wasn't significant.

While within a contemporary medical frame of reference, pregnancy at age 44 is incontrovertibly deemed 'high risk', Wilma's comments signal an alternative conception of appropriate fitness for birth, one which foregrounds physical and mental self-knowledge as key resources. In a similar vein, Marama emphasized the benefits of past experience and older age as a source of embodied knowledge, and stated that with each pregnancy she shed her 'buy-in' to a medicalised view of childbirth. Noting that her third pregnancy had not been screened, and had culminated in a planned homebirth, she commented:

I just kind of felt like I was that much older, and a little bit more aware of my body ... Being a bit older you're a little bit more, I was more aware of my own body, and I just sort of felt that's the way I wanted to go.

First-time mothers such as Anna and Rona also drew on non-medical sources of knowledge in accounting for their resistance to categorisations of risk. In spite of her high-risk designation following an abnormal scan result, Anna's personal evaluation told another story. As she stated, "I just felt like I was carrying a healthy baby".

\footnotetext{
${ }^{95}$ This notion of 'embodied knowledge' relative to pregnancy experience is also discussed by Press \& Browner (1996), Rapp (1998), and Lippman (1999).
} 
Similarly, Rona's rejection of screening (which will be discussed later in the chapter) signified a refusal to engage with a risk-based ontology of pregnancy. As she stated simply, "We just thought (baby's name) would be alright"; in her view, external verification of wellbeing was not required. Her explanation signals a reliance on an intuitive understanding of the status of her pregnancy and a conception of health and wellness which sits outside medically oriented understandings.

Such examples suggest that the use of embodied knowledge can be theorised as a moral resource or strategy through which pregnant women may account for their decisions by recourse to terms of reference other than the risk/reassurance binary of mainstream screening discourse. These accounts also support the previously made suggestion that for some pregnant women, the qualitative character of their pregnancy experiences (that is, the extent to which the experience 'measures up' in line with their personal moral world) is intrinsically linked to their evaluation of what constitutes a healthy, normal pregnancy and a good outcome. Variables such as the ability to determine for oneself such parameters as 'fitness' to give birth, and the best place for birth to occur are, for many of these women, directly relevant to the extent to which a pregnancy is evaluated as a positive experience. These experience-based insights are an important reminder of the potential tensions between lay and medical accounts of health, and the expectational rifts which may open up where little space is left for alternative readings of pregnancy other than as a functional event. The distressing potential of this disjuncture is well illustrated in Anna's experience (as discussed in chapter three): while Anna is relieved that her son's extra digits were not detected during an ultrasound scan, her obstetrician/sonographer is "gutted" by a missed diagnostic opportunity. From a medical perspective, pregnant women's 
resistance to diagnostic imperatives and clinical forms of knowledge presents a radical challenge to the presumption that a responsible mother will always put the needs of the unborn above her own (Rudolfsdottir, 2000). Sentiments such as those expressed by Kiri when she states "I really believe in the body's ability" confound mainstream expectations of pregnant subjectivity, along with an array of broader cultural discourses around risk and control. As Rudolfsdottir (2000) suggests:

pregnant women do not necessarily position themselves in terms of maternal normativities. Their accounts of pregnancy and childbirth both support and challenge the knowledge that underpins the practices of medical/healthcare institutions. (2000:337)

As will be revisited later in the chapter, it seems clear that those who accept screening, just as much as those who resist it, regard themselves as good mothers, making responsible choices.

\section{Time as a moral resource}

Drawing on findings from their recent ethnographic work on lay decisions around genetic screening, Scully et al argue for the need to recognize the significance of time in the context of individuals' moral processes. As they suggest, "To grasp the combination of intention, agency, behaviour, consequences and relationships that we characterise as moral responsibility means taking its constitutive temporality into account" (2007:208). A key finding of such work is that individuals facing genetic test results may variously manipulate their experience of time (for example by delaying undergoing tests they nonetheless wish to have in future), and that this is done in the interests of garnering 'moral space' sufficient to reach a personally acceptable decision. 
This resonated with Ruby's depiction of the state of 'suspended animation' she imposed on her pregnancy during a three-week wait for confirmation of an abnormal screening result (see previous chapter). In line with Scully et al's analysis, Ruby's deliberate restriction of her 'temporal depth of field' (2007:212) is readily interpreted as a strategic attempt to preserve a sense of control in the face of a protracted state of uncertainty. Melissa also describes consciously delaying an emotional connection with her pregnancy: "You have to keep that little distance - well I found I did - from pregnancy until you get to thirteen weeks anyway". These examples of temporal disconnection bear a similarity with women's accounts of a sense of disembodiment (see previous chapter), in the sense that both involve a degree of conscious disengagement with the physical realities of pregnant embodiment.

Press and Browner (1994) have framed pregnant women's widely-held view of prenatal screening as first and foremost a means of 'reassurance' (as opposed to diagnosis per se) in terms of an inchoate strategy to sublimate (or at least forestall) the dilemmas of selective termination. In this view, the generalized acceptance of prenatal screening reflects a willingness on the part of both women and clinicians to participate in 'collective fictions' about the purpose of screening and its therapeutic potential. In a similar vein, Williams et al argue that the framing of screening as a 'formality' of pregnancy care allows women to "bypass moral work" (2005:1987). This deliberate non-engagement with the various clinical and moral 'realities' attached to screening could readily be interpreted as a morally passive response; however I would argue that it could also be read as a moral strategy involving the manipulation of time. In this case it is not so much that time is 'bought' as denied, as 
a means to cope with the requirement that difficult decisions be made. I would suggest that the choice not to make a decision nonetheless should be recognized as within the spectrum of the unavoidable moral work pregnant women do within the context of routinised prenatal screening. It seems reasonable to argue that the fact that women may feel it necessary to act in this way to deflect the burden of decisionmaking requires that clinicians and policy-makers pay critical attention to the ethics of a 'public health' model of prenatal screening, where high 'uptake' rates are necessarily viewed as the optimal outcome. ${ }^{96}$

It is interesting to consider another possible example of pregnant women's use of time as a moral strategy. Within the New Zealand context, it is well recognized by maternity practitioners that a roughly consistent percentage of pregnant women do not seek prenatal care until after the first trimester (Essex et al, 1992, Low et al 2005). Given that these women consequently enter the maternity care system too late to undergo prenatal screening, yet nonetheless do seek prenatal care, it seems worth considering the extent to which late booking may reflect a deliberate choice to avoid the dilemmas of prenatal screening. Campbell (2008) notes that midwives are familiar with this form of selective engagement with prenatal care, and that the wish to avoid screening is accepted within the context of a 'women-centered' philosophy which underpins midwifery care. However, within obstetric literature, late booking is generally accounted for in terms of structural barriers to access to prenatal care such as financial and educational constraints, and a lack of social support, ${ }^{97}$ rather than in terms of individual choice or preference. Within this literature, an overall emphasis on the significance of information (or, specifically, the lack of this) for women's uptake

\footnotetext{
${ }^{96}$ The ethics of the application of a 'public health' model to the provision of prenatal screening will be discussed further in chapter five.

${ }^{97}$ See, for example, Essex et al (1992) and Young et al (1989).
} 
of screening suggests that within this domain a decision to avoid prenatal screening is not, by and large, recognized as a considered choice. For example, as the $1999 \mathrm{New}$ Zealand Review of Maternity Services notes, "Frequent late registrations with LMCs ${ }^{98}$ suggest that women may be receiving insufficient or poor-quality information about care options" (1999:5).

Rapp (1998) and Lewando-Hundt et al (2000) suggest that where women selectively engage with prenatal care, this can be seen to reflect a strategic attempt to balance the social and medical aspects of 'risk'. In line with this analysis, late booking for prenatal care could be interpreted as a strategy through which individual women access technologies such as ultrasound during the course of their pregnancy (and thereby fulfil social expectations of responsible motherhood) but avoid the diagnostic imperatives attached to the use of ultrasound in the first trimester (e.g. NTS) ${ }^{99}$ In this respect the choice to book late for prenatal care could be seen as an attempt to impose a 'therapeutic' rather than diagnostic frame on engagement with prenatal care.

Both internationally and within New Zealand it is clear that ethnicity plays a significant role in shaping women's engagement with prenatal screening. New Zealand data indicates that both Maori and Pacific women are significantly more likely to book late for prenatal care than Pakeha/New Zealand European women. Essex et al (1992) cite a 1990-91 study indicating that $28.9 \%$ of Maori women and $42.9 \%$ of Pacific women surveyed had not sought prenatal care until after thirteen weeks of pregnancy (too late for NTS). In addition to the tendency to book late, Low

\footnotetext{
98 "Lead Maternity Carers". In New Zealand the care of pregnant women undergoing normal pregnancies is now generally overseen by one practitioner with primary responsibility for the provision of pregnancy and birth services. This will be either a midwife, obstetrician, or (now rarely), a general practitioner (GP).

${ }^{99}$ Following the first trimester the use of prenatal ultrasound shifts from a diagnostic focus to the tracking of milestones of fetal development.
} 
et al (2005) note that Pacific women attend fewer prenatal visits than other ethnic groups. ${ }^{100}$ To date there is no research available which investigates the attitudes of Maori or Pacific women towards prenatal screening, hence it is not at all clear how these women themselves account for booking late for prenatal care, and whether or not this phenomenon reflects a deliberate decision. Given the significance of churchlinked traditionalism within the Pacific community in New Zealand it is possible that a reluctance to undergo screening may be related to religious sanctions against abortion. However, it is also interesting to speculate how the decision to avoid prenatal screening by booking late may reflect broader cultural expectations around pregnancy and birth. While Maori and Pacific peoples are statistically overrepresented in measures of poor health, it is worth noting that in comparison to women of European or Asian decent, Maori and Pacific women in New Zealand have significantly higher rates of normal, non-interventive birth (Johnson et al, 1995, Sadler et al, 2002).

This may possibly reflect a cultural aversion to technological interference with what is perceived as a natural and normal process, and it may be that this could perhaps account for a reluctance to participate in prenatal screening. The link between good birth outcomes and a view that birth is a normal physiological process is well recognized by birth practitioners such as midwives (Pairman, 2006); perhaps the high rates of normal birth and low rates of prenatal screening among Maori and Pacific women are linked by a negative view of the value of technological intervention. It is not within the scope of this thesis to specifically investigate the significance of ethnicity for women's screening decisions, however, the paucity of research available

\footnotetext{
${ }^{100}$ As Low et al (2005) note, there are no national guidelines for the recommended number of prenatal visits in New Zealand, however RANZCOG recommends that first time mothers attend at least nine visits, and women in subsequent pregnancies attend a minimum of six.
} 
within the New Zealand context suggests that there is considerable scope for research into the 'moral worlds' (Frank, 1997) of Maori and Pacific women relative to prenatal screening. Such research may add weight to the suggestion that the refusal of screening requires a more nuanced understanding of agency than that presumed in contemporary models of informed consent, and require that pregnant women's "noshows" for prenatal screening (Rapp, 1998:54) are not, by default, viewed simply as an epidemiological challenge.

\section{Metaphor as a moral resource}

Rapp (1998) noted the use of 'orienting metaphors' by religious women in their response to the diagnostic imperatives of prenatal screening (1998:64), although she did not extend this analysis more broadly. In more recent work on lay responses to medical technologies (specifically, genetic technologies), Scully et al (2006b) have discussed the use of metaphor in making sense of the experience of screening, and as a tool for articulating (to the self and to others) the various moral bases of individual decisions. The authors' observations about the conceptualisation of children as 'gifts' rather than commodities resonate strongly with the accounts of several women in this study.

For example, $\operatorname{Kiri}^{101}$ remarked that had screening produced a positive result for Down syndrome (rather than an incorrect negative result) she nonetheless would have proceeded with the pregnancy; as she stated, "(I) would accept any child that is given to me". Rona had a similar conception of children as gifts, expressing strong distaste for the notion of a 'selective' approach to pregnancy:

\footnotetext{
${ }^{101}$ As discussed in chapter three, Kiri gave birth to a child with Down syndrome subsequent to a negative screening result.
} 
You know there was a time when we thought having a girl was a problem, and now currently we think other things are a problem. What about the radical idea that it's not a problem, and what that would mean? Yeah because then people during pregnancy rather than thinking "am I doing the right thing, am I producing a good-enough child?" would be thinking "wow, I'm in this incredible connected state, I'm like a vessel for bringing a soul to earth, and that's beautiful.

Alosina similarly expressed concern that the targeting of specific disabilities had shifted cultural expectations around children from gifts to commodities, stating that "it creates this idea that, well, you deserve a perfect child, rather than we are given what we get".

The metaphor of the gift was frequently expressed in narratives of acceptance and in notions of surrendering and 'going with the flow' of pregnancy. As Kiri commented:

I think that the journeys of life shouldn't be given to you before your child is born. I think life is a gift that you need to ... it's an unknown path that you need to go on with your child.

Alosina similarly drew on the metaphor of a 'journey': "I feel like once the pregnancy's taken hold, then hey let's go, we just carry on, you know. We can't try to mess with it after". For Rona, acceptance of a child, irrespective of genetic status, embodied what she appeared to regard as a fundamental moral obligation of parenthood. Furthermore, in her estimation, a lack of unconditional acceptance not only signalled a lack of respect for the dignity she believed to be the due of the unborn, but also amounted to an act of emotional violence. ${ }^{102}$ This conception of unconditional acceptance as a moral requirement of good mothering was common to many women's accounts, including that of Portia, who had undergone a termination

${ }^{102}$ See quotation, chapter three, page 86. 
of pregnancy for Down syndrome. As she commented "the best parents are the parents who say this baby is going to be who this baby is going to be". As Taylor (1998) has argued, such tensions between the imperatives of unconditionality (i.e. as emblematic of 'good mothering') and provisionality (i.e. selective screening) appear to generate an intractable experiential paradox in many women's experience.

In articulating this metaphorical notion of gift/acceptance, a number of women drew on essentialist ideas relating to a 'natural' order to express their beliefs around the ethics of technological intervention. For example, Kiri's account suggests a conception of the instinctual nature of maternity; within this view, the unconditional acceptance of a disabled child appears to be a requirement of a kind of "natural morality' of motherhood:

I'm really happy that I didn't know. When I gave birth to him I had the opportunity of having the natural maternal instinct as well. That would be held back, I feel, if in preparation of having a child with a condition you would probably block the maternal instinct and deal with the condition, and then it would probably come out later, I would think. But I think that's one of the advantages of not knowing, that you just accept your child for what they are. And it naturally comes, that you do just accept your child as they are.

Similar to Kiri, Eva expressed a concern that screening interfered with beneficial natural processes: "I think the screening thing is interrupting what would normally happen ... the process is interrupted". Eva had experienced the birth and death of a genetically disabled baby. While prenatal screening had revealed a condition 'not compatible with life', her account of the decision not to terminate the pregnancy indicated a valuing of what she perceived as "natural processes" above the avoidance of suffering: 'I just think it's a natural process, and we have the ability in us, as step 
by step. And it might look terribly disastrous. But yet as the process goes, it can be ok'.

Eva's understanding of health appears to be implicitly linked to the acceptance of this conception of the processual nature of coming to terms with difficult experiences. As she states: "that's health too, when you go through the process". Within a number of accounts, a concern about the eugenic potential of selective screening signalled a conceptual link between health and genetic diversity. As Helen stated: "You're not going to get the perfect child, you know, or a perfect society, that's got no diversity in it". And Naomi:

I think we need a varied population, because everyone ... the balance of people in society affects how we interact, and I think, Oh I don't want to say just a 'PC' thing that they're all valuable, but, they teach us surprising things I guess, yeah, I just don't like the thought that we might be influencing our society in the future in ways we don't understand now. I just think that's really dangerous.

Comments such as these suggest a broad conception of health, within which disability appears to be valued as an aspect of broader societal wellbeing. This positive evaluation of disability was also reflected more generally within the study sample, irrespective of participants' personal screening decisions.

The acceptance of disability as a 'social good' is radically at odds with the trend towards a genetic orientation in contemporary models of health, and such findings indicate the possibility of a significant moral gulf between lay and medical attitudes. Disability theorists have long argued that medical 'deficit' evaluations of disability are out of step with public attitudes, and recent work on lay understandings of disability suggest that such views are formed with reference to a wider moral 
repertoire (Scully et al, 2004, 2006). Raz (2004) points to a cultural shift (at least within the West) towards the acceptance of disability, arguing that such acceptance reflects a broad ideological pluralism. In this analysis, the framing of disability as among an array of issues of 'identity politics' reflects the embeddedness of multiculturalism as a cultural value.

\section{Reinscribing clinical facts as social reality}

Several women within the study sample had given birth to babies with genetic conditions who were alive at the time of interview. Their accounts of the necessity to 'redraw' their offspring in non-medical terms highlight another aspect of the moral work attached to the experience of prenatal screening. Rapp usefully framed this in terms of "redescribing and reinscribing a powerful biomedical definition into the more complex and variegated aspects of personhood, childhood dependence, and family life" (1998:67).

As noted previously, Kiri, had received a false negative screening result, subsequent to which she gave birth to a child with Down syndrome. Virtually no ethnographic research exists on this phenomenon of 'false negative' screening results, and a specific investigation of the topic is not within the scope of this thesis. Nonetheless, Kiri's navigation of the tension between the various 'realities' - medical and social of her child's condition offers some useful insights into the implications of this experience, and the work involved in making sense of 'diagnosis' relative to personal experience. Kiri felt that while she made a rapid adjustment to her son's condition, the clinicians involved in his care treated him differently from other unwell babies. She explained that in her view, he was treated as a diagnosis, rather than a child with a diagnosis: 
Oh I realized very quickly the culture of his professional health, I mean he was in hospital for six weeks of his life, for the first six weeks of his life, and I knew right from then, I got a feeling of how his condition was perceived... I couldn't believe that us as humans still could see a human being as being different and still place them in there, in that box. You know it was very, very upsetting that these professionals still have that sort of feeling that they're different.

Kiri's account suggests the scope of the ontological work involved for parents of genetically disabled children in balancing the narratives of diagnosis and pathology attached to their children with the less conditional values associated with kinship and care. Such tensions are brought into stark relief in the following comment (Kiri):

I mean I have heard professionals, they have actually come up to me and said, you know, that it would be one of the best things in the world if they could find something that would stop the chromosome from splitting into three. ${ }^{103}$ You know? It's crazy, I wonder where these people are from.

These somewhat insensitive remarks by a clinician perhaps suggest the pervasiveness of a 'geneticised' model of health (Lippman, 1991) within contemporary medicine and raise a number of ethical questions about the downstream consequences of prenatal screening as a cultural norm. Where a condition is perceived as 'avoidable', to whom is responsibility attributed for the birth of an affected child? When such children are born, what are clinicians' roles in offsetting the genetic 'story' of that individual with parents' expectations that their child is valued equally to other children?

While clearly Kiri did not have the opportunity to terminate her pregnancy even if she had wanted to, the sense that her child is somehow not welcomed as other children

\footnotetext{
${ }^{103}$ Here Kiri refers to Down syndrome, also known as "Trisomy 21" due to the presence of three copies (rather than the normal two) of the twenty first chromosome.
} 
might be perhaps suggests that a subtly punitive subtext has become attached to contemporary screening practice. As suggested in chapter one, shifts towards a genetic orientation in healthcare appear to generate new moral normativities (Ettore 2002, Petersen \& Bunton, 2002), including the requirement to comply with projects of genetic health. However, as such compliance clearly does not necessarily guarantee the accurate detection of fetal abnormality, it is unclear where such 'responsibility' is deemed to lie. As suggested in chapter two, the question of technical failure and inaccurate diagnoses associated with current screening methods is substantially unaddressed as a matter of clinical ethics.

Kiri's account brings to mind Rapp's conception of pregnant women as a privatized moral workforce, absorbing the ethical tensions which society is reluctant to address. A number of new prenatal screening methods have emerged since Rapp's influential work on amniocentesis, including genetics-based techniques such as Pre-implantation Genetic Diagnosis (PGD). However, while genes may now be "public entities whose existence cannot be ignored and with which everyone has to reckon" (Callon \& Rabharisoa, 2004:10), it seems likely that with respect to pregnancy screening, it will continue to be individual women, rather than the public at large, who will be relied upon to privately navigate the ontological gulfs which arise in this meeting of diagnostic imperatives and lived experience.

The tensions between medical and personal expectations which are described in Kiri's experience can also be seen in Anna's account of a clinician's disappointment over a missed diagnostic opportunity during her scan; ${ }^{104}$ while her obstetrician is "gutted", Anna comments that "we were thrilled they didn't know during pregnancy". As

\footnotetext{
${ }^{104}$ See also previous chapter.
} 
discussed in the previous chapter, Anna's positive evaluation of her child's health status, in spite of the ongoing problems associated with his abnormality, illustrate her reinscription of a genetic diagnosis into a more positive framing which fits with the reality of her son's day-to-day progress. This personal reframing of clinical 'stories' was also evident in Eva's account. Eva had decided to continue her pregnancy after diagnosis of a lethal fetal abnormality, and the baby died shortly after birth. For her it seemed important that this pregnancy experience was not defined by a diagnosis, but rather the birth of a baby, irrespective of health status. As she said, 'I'm really pleased to have had that baby and given the baby the dignity of being a baby, and for us to hold him and have the dignity of a burial". Arguably, from a clinical perspective, the choice to continue a pregnancy to term and give birth to a baby that will inevitably die at birth, rather than terminate an abnormal fetus at an earlier point, makes little sense. However, in terms of Eva's experience, this decision allows for the transition of fetus to baby, and a subsequent, if brief, acknowledgement of kinship and parenthood. In resisting the usual clinical pathway, Eva effectively redirects and redraws her experience in line with her own moral frame. Eva's account resonates with Scully et al's contention that lay people making decisions about their own medical care seek to frame such decisions in a way which fits their inner moral worlds. As they suggest, the achievement of a personally acceptable experiential account may be at least as salient as the decision itself:

From the point of view of the patient, her primary ethical responsibility is not to make the ethically correct or ideal decision, but to find a solution that she believes, from past experience, can be part of her moral biography of the future. (2007:217). 
Eva's comments suggest that while she felt able to accept the inevitable death of her baby soon after birth, the termination of that pregnancy was not a decision which she felt she would be able to live with in the long term.

These accounts foreground the work involved in balancing diagnostic and personal narratives, and, in particular, in "reducing the significance of chromosomes to welcome a child on other grounds than biomedical normalcy" (Rapp, 1998:67).

\section{Experiential projects of pregnancy}

A number of participants described a sense of having been deprived of a positive pregnancy experience by their experience of prenatal screening. As noted elsewhere, at least four participants in the study stated that as a result of the negative aspects of their experience, they would be unlikely to undergo screening in a future pregnancy. This raises interesting questions about the extent to which the valuing of specific experiential goals, such as the enjoyment of pregnancy, and the preservation of an unexamined pregnancy, might have significance for women's screening decisions.

Warren and Brewis (2004) have theorized pregnancy experience in terms of being a particular kind of 'body episode', to which women may attach quite specific expectations of pleasure. For example, they found that some women viewed pregnancy as a chance to 'luxuriate in their materiality' (2004:219), and that pregnant embodiment was valued as a reprieve from the exacting physical norms of the contemporary feminine body project. Similarly, Young has conceptualised pregnant embodiment as an opportunity for women to reappraise their body in a more positive, less conditional light, and to "take an interest in its sensations and limitations for their own sake, experiencing them as a fullness rather than a lack" (1990:65). This notion 
of pregnancy as an experience buffered from external bodily norms resonates with Rona's wish to conduct an unexamined pregnancy. Rona's conception of pregnant embodiment seems to be of an otherworldly experience which ought to be savoured for its own sake, and not subjected to external expectations. In her view, the cultural imperative of prenatal screening made the prospect of pregnancy generally less pleasurable for women; as she stated: "You've got away from the idea of it's necessarily a joyous thing". Accordingly, she had made the decision to decline the offer of screening.

Several other participants who similarly had not followed the standard screening pathways also accounted for this decision in terms of a valuing of a pleasurable experience over diagnostic imperatives. For example, Margaret, who had declined the recommendation of an amniocentesis: "I just wanted to enjoy that time and not be stressing out about it". Similarly, Anna's account of a 'high-risk' pregnancy suggests that the preservation of a positive experience was at least as compelling as compliance with protocols of clinical management. Her lingering sense of having been "cheated" out of an experience she had wished for speaks of a sense of entitlement to a particular pregnancy experience.

Rudolfsdottir (2000) has argued that the qualitative character of individual pregnancies matters a great deal to the women experiencing them, and the extent to which these experiences are viewed as positive or negative is directly linked to a sense of agency in the context of prenatal care. The valuing of a positive pregnancy experience within these accounts suggests that the significance of pleasure may be underdetermined in current evaluations of factors which shape women's' screening 
decisions. I would suggest that this weighing of the expectation of pleasure relative to clinical imperatives should be recognised as another aspect of the moral work undertaken by pregnant women.

\section{Refusing prenatal screening}

Previous discussion has considered the ways pregnant women variously 'refuse' prenatal screening, in the sense that they engage with it on a selective basis. Within the study sample, four women indicated that while they had undergone ultrasound scans, they had declined a recommendation to undergo amniocentesis. In contrast to this selective approach, the accounts of two participants can be seen to signify a more wholesale refusal of prenatal screening. Rona and Marama chose not to undergo ultrasound scans (or any other screening method) during one pregnancy each. Given the widespread cultural acceptance of scans as a norm of prenatal care, their choices are taken, within the context of this study, to constitute a more radical rejection of prenatal screening than, for example, the decision to decline an amniocentesis. Their accounts provide a further platform for exploring the diverse moral work undertaken by women in their screening decisions. As Rapp's work suggests, the core tensions of mainstream screening discourse are crystallised within the 'commentary of refusers'; such narratives therefore offer a unique resource for exploring the parameters of what pregnant women consider to be at stake in their engagement with prenatal screening, by foregrounding "the cultural contradictions involved in the technological transformation of pregnancy" (1987:113).

Press \& Browner (1996) argue that against the backdrop of routinised screening, the refusal of screening necessarily constitutes an active, deliberate decision. However it is clear that irrespective of an emphasis on choice and consent within mainstream 
screening discourse, the decision not to screen is, by and large, neither popularly accepted nor medically sanctioned as a 'choice'. As noted earlier in the chapter, medical accounts of the 'non-uptake' of screening tend to disregard individual volition, focusing on structural 'barriers' to screening. ${ }^{105}$ In addition, as Green \& Statham (1993) and Press \& Browner $(1997,1998)$ argue, current clinical literature signals a long-held assumption that women's negative evaluation of prenatal screening is primarily linked to a moral objection to abortion. ${ }^{106}$ This view appears to persist in spite of more recent research highlighting the significance of women's concerns about the technical reliability and personal utility of available tests for their decision-making. ${ }^{107}$

The handful of qualitative studies which specifically address the question of refusal of pregnancy screening ${ }^{108}$ emphasise the fact that women in fact rarely decline prenatal screening, whether or not they consider it worthwhile. As Press and Browner (1996) observe, even where women indicate that they would not terminate following an abnormal result, uptake rates are uniformly high. Rapp's study on the refusal of amniocentesis (1998) found that most refusals occurred either during or immediately following a genetic counselling session, which suggests that such decisions were made with direct reference to information about screening, and therefore could be

\footnotetext{
${ }^{105}$ See for example Berne-Frommel et al (1984) and Jorgenson (1995).

${ }^{106}$ There is very little popular literature which deals with the experience of refusing prenatal screening. However, it is interesting to note that one recent example, Tankard-Resist's "Defiant Birth: Women who resist medical eugenics" (2006), similarly accounts for the refusal of screening primarily in terms of moral sanctions against abortion.

${ }^{107}$ For example, a study of women's reasons for accepting or refusing prenatal screening (Van den Berg et al, 2005) found that objection to abortion was the reason least frequently given by women refusing screening, with $42 \%$ of refusers citing 'unfavourable characteristics of the screening test' as the main reason the test was refused; such concerns related specifically to the reliability of the test and to the personal utility of a probability-based result.

${ }^{108}$ In particular see Rapp (1998) and Press, Browner \& Markens (1999), and for an example of recent clinically-oriented research in this area see Van den Berg et al, (2005).
} 
seen as 'informed'. Such findings present a challenge to medical accounts of refusal. What then are the bases of these refusals? This will be discussed shortly.

In foregrounding the accounts of pregnant women themselves, social scientific research has signalled the need for a more nuanced understanding of women's screening choices, and one which acknowledges the significance of individual agency in women's response to prenatal screening. Recent ethnographic work on the social and ethical implications of prenatal screening continues to cite Rapp's analysis of pregnant women as 'moral pioneers', and more recently a small amount of research has revisited this notion in light of newly developed, high-tech screening methods; specifically, NTS. ${ }^{109}$ However I would argue that there is still much scope to extend Rapp's exposition of the refusal of screening as emblematic of the privatised moral work undertaken by pregnant women, and in particular to explore the ways individual women account for this decision relative to their inner moral worlds. In particular the refusal of ultrasound screening specifically (as opposed to more invasive methods) is an area which is yet to be researched.

As this study did not focus specifically on women's refusal of screening, there was insufficient data to address this experience in great depth. However Marama and Rona's accounts offered some interesting insights into women's refusal of prenatal screening, and in particular the ideological bases of these refusals. Marama described how she chose not to undergo screening in her third pregnancy. As discussed earlier, she appeared to account for this decision partly in terms of a reliance on embodied knowledge, drawn from past experience of pregnancy and a sense of physical self-

\footnotetext{
${ }^{109}$ See, in particular, Williams et al, (2005).
} 
knowledge. In addition, her explanation for refusing screening signals a personal moral framework which sits outside the normative expectations of mainstream screening discourse. As she stated simply, "it wasn't really my thing”. Marama was strong in her opposition to routine pregnancy screening, which she described as "nutty". In her analysis, prenatal screening reflected a misguided and unhealthy exercise in risk-management:

I think it's mental, and I think it's a reflection of society's lifestyle, you know, convenience, and that bad things shouldn't happen. But I think it's a good thing that stuff happens in people's lives that you can't, you know, control.

In accordance with this evaluation, Marama accounted for her refusal in terms of a rational decision to opt out of an ideologically unpalatable social project. In a similar vein, Rona's explanation of refusing screening also signified the rejection of cultural norms of risk and consumption. In her view, prenatal screening was emblematic of a broader project of social uniformity, which she described this way: "(It's) a sort of fascistic idea of what everyone's life is supposed to be like, and what everyone is supposed to be like. And I think that's just totally degrading humanity". As her rationale for declining screening succinctly suggests, discourses of risk and reassurance have no personal resonance for her - "I just sort of thought I don't really see there's anything to be gained".

In their discussion of biomedical refusal, Callon \& Rabharisoa (2004) note that patients' refusal of medical technology has historically been interpreted as either an irrational rejection of medical beneficence, or as evidence of an amoral worldview. However, these readings, they argue, do not acknowledge the extent to which such refusals may reflect an áctive moral position in which a person may "opt positively 
for other forms of agency" (2004:22). When framed this way, Rona's refusal can be interpreted as an active rejection of a subjectivity of risk. Her decision to have an 'unscreened' pregnancy arguably constitutes a political act - she refuses to engage with or literally 'embody' the values which she rejects, and in this way preserves a morally acceptable sense of herself as an agent. Rather than morally 'opting out', Rona makes her choice with reference to "a different form of morality" (Callon \& Rabharisoa, 2004:1).

\section{How are 'refusals' medically and socially judged?}

Mainstream medicine has traditionally framed patients' refusals of therapeutic advice and intervention in terms of 'non-compliance' or deviance, an approach which signals a presumption of the authoritativeness of clinical knowledge (Donovan \& Blake 1992, Lupton 2003). As Lupton (1999) has also argued, the management of contemporary pregnancy expresses an 'ontology of risk', and in pregnant patients such refusals have tended to draw an additional dimension of moral scrutiny, in line with normative cultural expectations of appropriately risk-averse maternal behaviours, including healthy eating (Copelton, 2007) and abstinence from alcohol (Armstrong, 1998).

Where women's refusal of prenatal screening is (minimally) addressed in the clinical literature, typically this is not framed in terms of moral lack or 'deviance'; this is perhaps unsurprising, given the rhetorical emphasis on consent and choice within contemporary medicine and the public health domain. However, while the medical rationale for screening is generally subsumed within public health discourse by narratives of individual choice, control and reassurance, ${ }^{110}$ it is nonetheless clear from clinical literature that women's refusal of screening is broadly viewed within this

\footnotetext{
${ }^{110}$ This is discussed further in chapter five.
} 
domain as an epidemiological challenge, rather than recognized as potentially a considered choice.

There is limited research available which might indicate how clinicians themselves respond to women's refusals within the clinical setting. However, it seems reasonable to argue that in making screening routine, the morality of acceptance or refusal of screening is likely to be judged negatively by practitioners and lay people alike. This is borne out in studies indicating that women who declined screening, or continued a pregnancy after positive diagnosis, were attributed responsibility for the birth of disabled children, and viewed as less deserving of emotional and social support than other women (Marteau \& Drake 1995, Lawson 2003). This attribution of responsibility was observed both in clinicians and lay people. Such findings lend considerable weight to the argument that the choices made available by prenatal screening are highly contingent ones; as Lawson suggests, "it is unlikely that women are unaware of or unaffected by these attributions and their repercussions" (2003:86).

While further research would be useful in gauging the depth of 'punitive' social and medical judgment around decisions to decline prenatal screening, women's experiences and attitudes within this thesis certainly suggest that a weighing of both the material and social implications of raising a disabled child may be a significant aspect of the decision-making process. Where the question of disability support arose during interviews, participants commonly characterised this as an under-resourced area of health care and social support, also frequently mentioning negative societal attitudes towards disability. It is not therefore difficult to imagine the potential significance of this awareness for women facing the decisions to terminate an 
abnormal pregnancy, particularly where women may be economically vulnerable or lack family support.

To return to Marama's and Rona's decision to decline screening; in each case, this choice had not been queried by their maternity caregivers, and as both women suggested, this seemed likely to be related to their selection of midwives prepared to accept this decision as a right of informed choice. However, the accounts of a number of other women who variously resisted screening during their pregnancies (in particular, Anna and Wilma) suggest the sense of normative pressure attached to clinicians' endorsement of screening as a norm of prenatal care.

The choice to decline prenatal screening flies in the face of an array of intertwined normative expectations attached to pregnant women's identities. The accounts of Marama and Rona foreground the significance of 'non-scientific' understandings of pregnancy in women's decision-making, and suggest the need to recognise that such decisions may in fact be made relative to a variety of ontologies of pregnancy and pregnant experience. 


\section{Conclusions on the analysis of interview data}

In exploring the accounts of women who refuse or engage selectively with prenatal screening, this chapter has considered a realm of experience which lies beyond the parameters of mainstream screening discourse. Close attention to women's own accounts of screening decisions reveals the significance of individuals' 'moral worlds' (Frank, 1997) in shaping this process. These accounts draw attention to multiple tensions between the mainstream 'story' of prenatal screening, and the embodied experience. The experiences foregrounded in the empirical component of this research problematise the widespread assumption that prenatal screening exists primarily as a response to women's 'need' (Lippman, 1991), and shows that women themselves may question the extent to which such a need may be, as Lippman suggests, a constructed one. This chapter has considered the nature and scope of the moral work attached to women's screening decisions. It seems clear that for some pregnant women, the achievement of both a sense of a satisfactory level of autonomy during their pregnancies, as well a personally-acceptable "moral biography of the future" (Scully et al, 2007) involves the need to actively resist medically and culturally prescribed ontologies of pregnancy.

The fact that some women choose 'against the grain' in this way indicates that pregnant women may attach a variety of aspirations to the experience of pregnancy which sit outside clinical and cultural norms. That is to suggest that the production of a healthy, genetically normal infant may not, contrary to dominant discursive constructions of responsible mothering, be the only thing women seek to achieve out of their pregnancy experiences; to draw on the metaphor used by a number of participants, the 'journey', and not just the 'destination', seems to be important. These accounts show that over and above the wish to 'conduct' healthy pregnancies 
(through, for example, correctly embodying the developmental norms of fetal growth, and compliance with imperatives of a risk-management model of pregnancy care) for some women, pregnancy is viewed as an opportunity to live out or literally embody a number of values and experiential goals.

It seems clear that it is not only women who decline screening who draw on nonmainstream ontologies of pregnancy. While the acceptance or rejection (or in clinical terms, the 'uptake'/'non-uptake') of prenatal screening is readily interpreted as signifying opposing attitudes toward prenatal screening, analysis of participants accounts suggests that it may be more appropriate to conceptualise these choices, as Markens et al have suggested, in terms of a "continuum between full acceptance and complete rejection of medical authority" (1999:361). They caution against the presumption that acceptors and refusers of prenatal screening may be viewed as "two meaningful and distinct groups" (368). As Rapp suggests, "Those who refuse the test, no less than those who accept it, are ... responding to a complex, highly structured social nexus within which they negotiate and exercise personal choice" (1998:620).

Callon and Rabharisoa have argued for the need to 'be attentive to local moral options in their confrontation with morals that claim universality' (2004:24). As chapter five will suggest, contemporary models of patient agency fall short of recognising the variety of moral frames which shape the decisions of individual lay people. The participant accounts discussed in this and the previous chapter offer evidence to support the argument made in this thesis that current policy and practice in prenatal screening continues to underdetermine the moral, psychological and emotional significance for pregnant women of this 'everyday' experience. Responding more 
generally to such examples of disparities between the various official and unofficial 'stories' of screening practices, Scully et al have recently argued that "bioethics itself needs to become more knowledgeable about the concerns, values and forms of reasoning that lay people bring to their ethical deliberations" (2006:764). The framing of women's prenatal screening decisions as 'moral work', as this chapter has done, draws attention to these private deliberations, and creates a space for clinical ethics to respond more sensitively to what is, for many women, a complex and ambivalent choice. The ways in which the various burdens associated with this choice might be pragmatically mitigated will be considered in chapter eight. 


\section{Chapter Five \\ Choice or compulsion? Ethical issues in prenatal screening: the question of informed consent}

This chapter considers the ethics of current practice in the provision of prenatal screening services, in particular evaluating the implications and appropriateness of a population-based, risk management approach. It is suggested that the constitution of prenatal screening as a public health concern raises a number of as yet unaddressed problems for clinical ethics, and in this light, the adequacy of existing mechanisms of ethical governance are considered. The implications of a public health model for the ethical integrity of protocols of informed consent are given particular attention.

Lupton (1997) and Scully et al (2007) have argued that moral decision-making as an individual experience has received little social scientific attention. I would suggest that while women's engagement with prenatal screening may appear to have been extensively investigated, with a substantial number of qualitative studies now having investigated women's motives for accepting or refusing screening, such studies have, as Garcia et al also suggest (2008a), typically focussed on social, psychological or religious determinants of decisions for and against screening. ${ }^{111}$ Such work has tended not to pay close attention to the nature of individual women's ethical beliefs, and the significance of these for their decision-making. Social scientific work which frames and investigates prenatal screening decisions in terms of the 'moral worlds' of individual pregnant women, is, as suggested in chapter one, a more recent

\footnotetext{
${ }^{111}$ Work of this nature cited in this thesis includes the studies of Press \& Browner $(1997,1998)$, Markens, Browner and Press (1999).
} 
development. ${ }^{112}$ In my view there is still therefore considerable scope for further work exploring the values, beliefs and intuitions which underpin pregnant women's screening decisions.

The inclusion of participants' accounts of their decision-making within this chapter responds to recent calls within health-oriented social scientific work to 're-embody' bioethics debates (Shildrick, 1997), that is, to assert the significance of embodied experience for ethical practice in healthcare. This perspective is grounded in a broader sociological movement which takes an interest in the subjective experiences and material practices associated with being in a body, or 'lived experience' ${ }^{113}$. Such work is underpinned by the view that a focus on 'experience' makes an important contribution to more traditional theoretical and methodological approaches to the study of health and illness. Elements of this perspective can also be seen to have been recently taken up within contemporary clinical ethics, where there is evidence of a reevaluation of the adequacy of the traditional 'principlist' model. ${ }^{114}$ For example, Frank (1997) and Charon (2001a, 2001b) emphasise the therapeutic benefits of a communicative rather than strictly principlist approach to ethical practice, for example stressing the value of subjective accounts within clinical education programmes as a way to encourage a more empathetic and reflexive practice style among health professionals. However, the subjective accounts of clinical encounters discussed in this chapter here suggest that calls to reconfigure clinical ethics in ways which better reflect patients' needs and wishes may be yet to have a substantive impact within the

\footnotetext{
${ }^{112}$ Although, as chapter one notes, Rapp's positioning of pregnant women as 'moral pioneers' in prenatal decision-making should be recognized as a key historical antecedent to this approach.

113 A term commonly cited in work drawing on the phenomenological approach of Merleau-ponty.

114 That is, a system of ethics based on abstract moral principles, such as beneficence (the imperative to do good) and non-maleficence (the imperative to do no harm). Traditionally, clinical (i.e. medical) ethics reflects this approach to ethical conduct.
} 
context of day to day clinical care, at least within the New Zealand context. As this chapter will argue, the evident persistence of a 'gap' between the theory and practice of informed consent for prenatal screening raises questions about the responsiveness of current ethical protocols to the complex reality of individual experience.

\section{Prenatal screening as an emergent 'public health' concern.}

As Williams et al (2005) and Lupton (1995) have noted, in recent years a shift from targeted screening to population-based, routinised approach signals a reconstitution of prenatal screening as a state-sponsored, public health activity. As noted previously, one consequence of this approach has been that increasing numbers of women undergoing normal (or "low risk") pregnancies are brought into engagement with a diagnostic field of medicine. In this respect, prenatal screening as a public health modality can be seen to blend a broad-based "catch-all" approach with an implicit risk orientation. The implications of this in terms of clinical ethics will be considered later in the chapter.

Within many countries, the increasing focus on diagnostic screening programmes has signalled a broadening of the public health remit, resulting in the channelling of significant amounts of state funding into activities of 'preventive medicine' (Petersen \& Lupton, 1996). Such paradigmatic shifts have seen the gradual alignment of notions of 'prevention' and 'therapy' within the activities of biomedicine, a phenomenon with obvious significance for prenatal screening.

In New Zealand, health screening programmes are overseen by the National Screening Unit (NSU), which at present co-ordinates screening for newborn hearing, vision and metabolic disorders, as well as breast and cervical cancer screening. The 
NSU's vision statement can be seen to encapsulate the therapeutic assumptions which, as Lupton (1995) suggests, underpin the activities of public health and health promotion: "Saving lives, reducing inequalities, and building the nation's health by leading the delivery of screening programmes, uncompromising in their quality and trusted by the communities we serve" (NSU, 2008, unpaginated). Within this conception of the NSU's role, prenatal screening can be seen to be positioned as one among a range of activities of 'therapeutic prevention' necessary for the general wellbeing of the community. A view of prenatal screening as a very significant aspect of this broader goal is well illustrated by the NSU's 2007 recommendation to government that a co-ordinated programme of prenatal screening for Down syndrome be implemented in New Zealand within two years. ${ }^{115}$

The constitution of prenatal screening as a public health project raises a number of ethical questions which relate to the role of medicine in society. Firstly, given the primarily therapeutic imperatives of public health as an operation of medicine, how well does prenatal screening fit within the remit of public health aims? Given popular assumptions of prenatal screening as a medically endorsed health benefit (Browner \& Press 1996, Davis-Floyd 1992), it seems reasonable to scrutinise what, technically speaking, the medical benefits of screening for fetal abnormality may be. Getz \& Kirkengen (2003) note that with respect to standard measures used in the rationalisation of prenatal health resourcing, that is, reductions in maternal and fetal mortality and morbidity, the overall medical benefit of routine screening is questionable. With respect to benefits for fetal health, as noted in chapter two, there are currently no therapeutic remedies available for conditions such as Down

${ }^{115}$ See chapter six for further discussion of the New Zealand situation. 
syndrome when these are detected prior to birth. Furthermore, it is recognised that prenatal screening does not elicit health-promoting behaviours in pregnant women. For example, clinical studies on the impact of prenatal ultrasound on women's smoking behaviour have shown that pregnant women's decisions to stop smoking are not linked to the experience of fetal scanning. ${ }^{116}$ Thus, it seems evident that the justification of prenatal screening on the basis of health promotion rests primarily on the presumed health benefits of selective termination.

Critics of routinised screening, and in particular disability theorists, have suggested that rationalising selective termination in terms of medical benefits is disingenuous, arguing that screening programmes are in fact driven primarily by economic rather than therapeutic imperatives (Alderson 2001, Shakespeare 1998). A review of epidemiological/public health literature concerning prenatal screening certainly gives some support to such claims, and this appears to be especially germane with respect to clinical discussion of Down syndrome in particular. ${ }^{117}$ It is worthy of note that "costbenefit' evaluations of Down syndrome rarely feature in literature on prenatal screening which is produced for popular consumption, which tend to emphasise the social and emotional costs for affected families. This may arguably be due in part to the difficulty of framing termination of pregnancy as a "health benefit"; as Clayton (1999) has argued, it is difficult to justify screening as a public health activity solely on the basis that it allows the selective termination of some abnormal fetuses. As she suggests, this may relate to the fact that while many people who are in principle

\footnotetext{
${ }^{116}$ See for example, Eurenius et al (1996).

${ }^{117}$ See for example, Beazoglou et al (1998), "Economic evaluation of prenatal screening for Down syndrome in the USA". The authors estimate that the average lifetime costs of an individual with Down syndrome are \$471,000 (as of 1992). Similarly, Vintzileos et al (2000) suggest that in the USA prenatal screening for Down syndrome potentially allows for annual savings of approximately $\$ 96,000,000$ per annum.
} 
supportive of access to abortion, nonetheless have a moral aversion to the notion of permitting a selective approach. ${ }^{118}$

It does not seem unreasonable to suggest that while the degree of disparity between popular and medical accounts of the purpose of screening is gradually coming to be acknowledged in terms of the costs for individual women, at a broader level questions about the appropriateness of a public health approach to the issue of fetal abnormality have received minimal ethical attention. Given public interest both in moral issues associated with healthcare (particularly those relating to reproductive technologies), and in the use of funds drawn from public taxation, an evident lack of public consultation to determine the demand for expanded prenatal screening services in New Zealand raises interesting questions about the valuing of public opinion within the public health domain.

A second key issue raised by the adoption of a public health approach relates to the question of what or who actually drives the current screening imperative. Critics of routine screening have questioned the extent to which prenatal screening fulfils a demonstrated 'need', arguing that further evidence is necessary to evaluate whether the provision of screening is driven by either demonstrated clinical benefit (i.e. as a requirement of 'evidence-based' medicine) or consumer demand. The requirement that health practices and research be directed by an 'evidence-based' approach is a key tenet of contemporary health policy (Bensing, 2000). This model seeks to ground medical decision-making in the most up-to-date scientific evidence (preferably randomised controlled trials, or RCTs) (Jonsen et al, 2006), and in tandem with the

\footnotetext{
${ }^{118}$ This is consistent with the view of the majority of those interviewed for this thesis.
} 
requirement for patients' informed consent is regarded as a key mechanism for safeguarding sound practice. However, as Marteau et al $(1992,2001)$ have argued, information provided to pregnant women about prenatal screening often fails to reflect a value-neutral, evidence-based approach, and is frequently directive. For example, in a 1996 study of patient information leaflets about prenatal ultrasound offered in 14 maternity units in the UK, only one leaflet was found to contain evidence-based information sufficient to fulfil the requirements of informed consent (Oliver et al, 1996). As the authors of the study note: "much of the information produced for users of health services describes health care processes without providing information about health care options and outcomes. Little is based on systematic reviews of research evidence" (1996:1253). This is particularly salient within the New Zealand context where although no available is data on the distribution of patient information leaflets, an 'ad hoc' approach to the provision of prenatal screening information to women by practitioners has recently come to be recognised as an issue of concern (Stone \& Austen, 2006). Certainly, as chapter six will show, it is clear that within the New Zealand context there is considerable variation between maternity practitioner types in terms of what written material, if any, is made available to pregnant women.

Interestingly, Oliver et al's study also noted a 'protective' attitude among practitioners (in this case sonographers) towards the release of clinical data into the public domain. This was the case even when such information was claimed to be being provided to patients in the interests of facilitating informed decision-making. ${ }^{119}$ As the authors

\footnotetext{
${ }^{119}$ It is interesting to note that in the course of this study, a request to RANZCOG for copies of patient information leaflets on prenatal screening for research purposes was declined on the basis that, in the interests of patients' informed consent, such information should only be made available to women within the context of the doctor-patient consultation.
} 
concluded, within the context of the provision of information to lay people, an evidence-based approach may be seen as a threat to professional autonomy. ${ }^{120}$ If, as will be discussed below, women rely significantly on their encounter with individual practitioners as the primary informational context of their decision-making, a phenomenon of professional "gate-keeping" such as that identified in this particular study would seem to have considerable implications for the achievement of informed consent.

With respect to the question of consumer demand for prenatal screening, critics have argued that medical agencies, rather than lay people, provide the main impetus for the implementation of screening programmes (Alderson, 2001). As Williams et al (2005) argue, prenatal screening techniques have historically been implemented in advance of demonstrated clinical benefit; this lack of public consultation in the development and use of genetics-based screening technologies is increasingly recognised in social scientific critique. ${ }^{121}$ The ethics of directing significant amounts of health funding towards practices which may not in fact be societally sanctioned has to date received little attention. As Alderson (2001) notes, the assumption within public health policies that screening necessarily reduces overall health spending deserves scrutiny; she cites a clinical comparative study of UK screening methods, which concluded that a routinised rather than targeted approach (in this case to maternal blood screening for Down syndrome) would likely detect only one more baby with Down syndrome per annum. As the study authors note:

\footnotetext{
${ }^{120}$ Such findings parallel chapter seven's discussion of sonographer's perception of their role, and in particular the threat seen to be posed to the authoritativeness of expert knowledge by the popular view of fetal images as easy to interpret.

${ }^{121}$ See also for example Mitchie et al (1995) and Kerr \& Cunningham-Burley (1998).
} 
The cost of preventing this one extra Down's birth would be one or two normal babies lost after amniocentesis, 4,500 blood tests for young women (with the associated anxiety and counselling), approximately 200 false positive serum test results and amniocentesis (with the associated anxiety and distress), and 90, 000 for the extra tests, counselling, and amniocentesis.

(Fletcher et al, (1995:351) cited in Alderson (2001: 362)

If it is indeed the case that prenatal screening is driven less by consumer demand or clinical benefit (i.e. is 'evidence-based') than economic rationalism, or that such rationalisations are based on questionable economics, this would appear to cast considerable doubt on the ethical justifiableness of constituting such screening as a public health endeavour. This aside, the potential psychological and emotional impact of a population-based model of prenatal screening raises further questions about the appropriateness of screening as a public health modality. These will be discussed now.

\section{Risk discourse in public health: ethical implications}

As argued previously, one of the most far-reaching consequences of a routinised approach to prenatal screening has been the conscription of all pregnant women within a 'risk-management' modality of care. Relative to this framework, every pregnancy is positioned along a spectrum of risk, irrespective of a woman's preexisting health status. Since Rothman's theorisation of 'the tentative pregnancy' (1986) sparked sociological interest in prenatal screening, the psycho-social ramifications of a risk-based model of prenatal care began to gain recognition in clinically oriented, interdisciplinary health research, particularly within the fields of psychology and genetic counselling. ${ }^{122}$ This and subsequent literature clearly expresses a concern that insufficient measures are taken within the clinical setting to

\footnotetext{
${ }^{122}$ See, for example, the work of Teresa Marteau $(1989,1993)$ and also Helen Statham and Josephine Green (1993, 1996).
} 
mitigate the psychological impacts for pregnant women of screening as a form of riskanalysis. This view is reiterated within literature endorsing a midwifery model of practice, where a connection is clearly drawn between a high-tech, risk-oriented approach to prenatal care and evidence of declining rates of normal birth throughout western countries. ${ }^{123}$ Within the Australasian context specifically, Reiger (2006) has suggested that an apparent 'crisis of confidence' among women about their capacity to give birth normally is a direct consequence of the climate of risk which, she argues, characterises contemporary pregnancy care. Such 'macro' analyses of the potential psychosocial impacts of prenatal screening are, generally speaking, rare in clinical literature. While obstetrics has gradually come to recognise the evident link between fear and anxiety about birth, and poor birth outcomes, the contribution and consequences of prenatal screening for an overall increase in maternal anxiety (and any associated morbidity), has not to date drawn significant clinical attention; as noted previously, prenatal screening is consistently described in clinical literature as beneficial to pregnant women, chiefly through the sense of reassurance it brings.

It would be extremely difficult to measure any substantive relationship between anxiety linked to prenatal screening and poorer birth outcomes per se. However, with reference to the experiential accounts analysed for this thesis it seems clear that women themselves sometimes regard anxiety associated with screening as having a negative impact at least in terms of the quality of their experience. For example, this is illustrated by Anna's account of being designated 'high-risk', which she felt had "cheated" her of the less stressful pregnancy experience which she could reasonably have had. Her insistence that the birth take place in a 'low-risk' setting with familiar

\footnotetext{
${ }^{123}$ See, for example, Wagner $(1994,2001)$.
} 
caregivers suggests that she felt her chances of a good birth outcome were higher in this setting than in a high-risk unit. In Anna's analysis, her experience illustrated the extent to which the intensification of prenatal screening may have contributed to an excessively 'anxious' and risk-averse culture among clinicians themselves.

Lupton (1995) argues that risk is the predominant organising discourse within public health models. However, it is significant to note the emergence of a counter-theme within maternity care discourse, specifically the promotion of a low-tech approach to birth. Within recent years, prenatal and maternity care has increasingly been designated within countries such as New Zealand as directed by a 'primary care' approach. Within the New Zealand context maternity care is specifically identified as within the remit of the government-led, nationally implemented, Primary Health Care Strategy (2001). As Pairman \& Guilliland (2003) note, a 'primary care' approach to the provision of maternity services in this country is seen as underpinned by a view of pregnancy and birth as, for the majority of women, normal life events. State endorsement of midwifery practice ${ }^{124}$ including full funding of midwifery services reflects an alignment with the World Health Organisation position that midwives are the most appropriate and cost-efficient caregivers for normal pregnancy and birth (WHO, 1996). The coexistence of these two models has generated a curious philosophical tension in the provision of maternity care in New Zealand, whereby practitioners can be seen to juggle imperatives of risk-management with the requirement for a non-interventionist approach. It could be suggested that recent revelations of the inconsistent, and "ad hoc" (Stone \& Austen, 2006) provision of prenatal screening services in part reflect this struggle to reconcile these contradictory

\footnotetext{
${ }^{124}$ Midwives in New Zealand have been able to practice autonomously since the passing of the Nursing Amendment Act in 1990.
} 
views of appropriate maternity care. While prenatal screening falls within the schedule of primary maternity services officially available to pregnant women, it is clear that a population-based approach to screening which designates all pregnant women as 'at risk' is at odds with the aims of a primary care approach. Given the increasing recognition of the individual consequences - both material and existential of a risk-oriented approach to prenatal care, it seems necessary to scrutinise the recent drive to implement a formalised public health model for prenatal screening within the New Zealand context. The specifics of the New Zealand situation will be discussed in chapter six.

\section{An alternative model}

While a broad-based 'risk-management' (i.e. routinised) approach to prenatal screening has now been adopted in the majority of westernised countries, in the Netherlands this is not the case, with screening offered on a strictly targeted basis. Within the Dutch maternity care system, a population-based approach to screening is viewed as posing a psychological and physical threat to both individual and societal wellbeing (van den Berg et al 2005, Garcia et al 2008a). Under the Population Screening Act (1996), ${ }^{125}$ universal screening for untreatable and unpreventable disorders is prohibited. As termination of pregnancy is not recognised in Holland as either a therapeutic or preventive practice, prenatal screening for fetal abnormality is not permitted as a routine practice. Prior to 2007 maternity practitioners were prohibited from offering prenatal screening to pregnant women unless they had an increased risk of fetal abnormality (i.e. advanced maternal age); since this time practitioners have been permitted to inform 'low-risk' women about available screening tests, however the cost of these tests must be privately carried (Garcia et al,

\footnotetext{
${ }^{125}$ Cited in van den Berg et al, (2005).
} 
2008a). Recognising the potential negative impacts of the spectre of risk on pregnant women's experience, and in particular the anxiety associated with private moral dilemmas (van den Berg et al, 2005), the Dutch approach to prenatal screening can be seen to reflect broader cultural values relating to the benefits of approaching pregnancy and birth as normal life events. A philosophical commitment to a 'primary care' model of birth is played out in state provision of fully funded midwifery services; similar to New Zealand, midwives practice autonomously, providing the vast majority of prenatal care across the general population (Tew \& Damstra-Wijmenga, 1991).

In acknowledging the ethical dimension of the provision of prenatal screening services, the Dutch system provides a useful working model of an alternative approach to universal screening as a 'default' public health position, and throws into question the self-evident logic of a population-based risk-management model. Furthermore, with respect to the current New Zealand approach, this model potentially offers a template for a more successful compromise between the somewhat contradictory aims of a primary care model of maternity care and the NSU's call for the expansion and national co-ordination of prenatal screening services. While potentially moderating the current risk-orientation of prenatal care in New Zealand, arguably adopting a version of the Dutch approach might also be viewed as satisfactory by women who do strongly identify with a risk designation in their view of pregnancy and birth (for example, Karla and Diane), given that in choosing private obstetric care, such women are presumably already inclined to find a way to fund 'extra' services above and beyond those provided by the state. 
Irrespective of any debates over the ethical appropriateness of a 'public health' approach to prenatal screening, it is clear that prenatal screening is now firmly established as a routine, state-endorsed practice in the majority of western countries. Discussion therefore will now turn to consideration of the adequacy of existing mechanisms of ethical governance in view of routine screening as a public health norm.

\section{Informed consent}

Widely viewed as the optimal model for the ethical provision of healthcare services, the principle of informed consent has, within the last twenty or so years, become an increasingly prominent feature of the landscape of contemporary health care. As a 'medico-legal' notion, informed consent is variously defined, dependent on context. Within the context of healthcare provision, informed consent is intended as the primary ethical and legal mechanism for protecting patients' right of autonomy and bodily self-determination (Beauchamp \& Childress 2001, Jonsen et al 2006, Ridley 2001). As such within the context of this discussion it is conceived as able to be regarded as a substantive measure of ethical practice in prenatal screening.

As Dahl et al, (2006) note, an increasing emphasis on patient autonomy as a measure of ethical practice reflects a shift away from the model of 'benevolent paternalism' characteristic of previous practice models. This shift has been precipitated within the last two decades by public inquiries into inappropriate and non-consensual medical practices (including, in the UK, investigation into the non-consensual removal and retention of children's organs at Alder Hey hospital in 1999). Within New Zealand, the notable emphasis within the health sector on the importance of informed consent similarly reflects the fallout of historic claims of medical misconduct. In the wake of 
revelations of the long-term, non-consensual and experimental treatment of cervical cancer sufferers at a regional hospital, the 1998 Cervical Cancer Inquiry recommended the establishment of a formalised complaints process for patients ('consumers') of health-care services in New Zealand. In 1994 the office of the Health and Disability Commissioner (HDC) was established, subsequently (in 1996) producing a code of patient rights guiding the provision of all health and disabilityrelated services within New Zealand. Within the code, the principle of informed consent is specifically addressed in Right 7 as 'the right to make an informed choice and give informed consent'. A number of other rights within the code signal the broader requirements of informed consent, chiefly 'the right to be fully informed' (Right 6) and 'the right to freedom from discrimination, coercion, harassment, and exploitation' (Right 2).

While the ascendancy of a 'patient-rights' focus in healthcare, and specifically, the protocol of informed consent, has been popularly welcomed as a protection against medical abuses, social scientific critique has drawn attention to the vulnerability of such protocols to being cynically utilised within the health sector as an 'ethical panacea' (Corrigan, 2003). Given the increasingly litigious climate of medical practice, it has been suggested that the expectation of patient consent has more to do with pre-empting malpractice claims than the protection of individual autonomy. In addition, the assumptions of individual autonomy which underpin the principle of informed consent are seen as lacking recognition of material and discursive constraints on individual agency. Such concerns have emerged out of a broader social scientific critique of bioethics as historically failing to be directed by (or even sensitive to) the contingencies of lived experience (Lopez, 2004), a lack of readiness 
to recognise the significance of bodily experience for individual autonomy (Bordo 1993, Frank 1990) and for lacking acknowledgement of the situatedness of its own discourse (de Vries, 2003). In challenging the notion of moral agency as a generalisable ideal, such critiques of a 'principlist' model of bioethics, draw on feminist ethicists' assertions that autonomy is context-dependent, and that individual moral agency cannot be abstracted from the material and ideological constraints which shape individual experience (such as differences in power and access to resources) and relational responsibilities (Young 2005, Shildrick 1997, 1999). A tendency to disregard individuals' embodied knowledge, belief systems and past experience has constituted a specific element of feminist critique in this area, which as will be discussed later, has particular resonance for women's decision-making around prenatal screening. ${ }^{126}$

A parallel (and sometimes intermingled) line of medical critique has raised questions about the practical difficulties (i.e. for maternity practitioners) of realising the ideals of informed consent within the clinical setting. ${ }^{127}$

\section{Prenatal screening: the problem with informed consent}

As noted previously, the manner in which women's informed consent for screening is problematised by an informational deficit is now well-recognised as a problem for clinical ethics. Chiefly this is seen to derive from the inadequate provision of information by practitioners, as well as a broader phenomenon of limitations within popular understandings of the purpose of screening. ${ }^{128}$ As chapters three and four

\footnotetext{
${ }^{126}$ This is also discussed in chapter four in relation to the decision to decline screening.

127 See later in the chapter.

${ }^{128}$ See for example, Kohut et al (2002), Marteau (1995), Santalahti et al (1998), Marteau \& Dormandy (2001) and Proud \& Murphy-Black (1997).
} 
indicate, such factors are similarly recognised by pregnant women themselves as issues of considerable concern. However, I would suggest that examination of pregnant women's own accounts of decision-making signals a number of additional obstacles to the achievement of authentic and informed consent for prenatal screening which are yet to be significantly acknowledged. These will now be considered.

\section{'Risk' and consent}

Lupton (1995) has argued that the ethical implications of the mobilisation of risk discourse within public health activities are yet to be practically addressed. In particular I would suggest that the significance of the relationship between risk discourse and consent is currently underdetermined in clinical ethics.

In a similar way that a public health approach to the provision of maternity care can be seen to sit uncomfortably alongside other existing models, so too are tensions evident in the constitution of screening as, at once, a 'choice' and a rational strategy of personal risk avoidance. Lupton $(1995,1999)$ and Petersen \& Bunton (2002) have argued that contemporary public health models privatise risk, educing a kind of enforced reflexivity which obliges individuals to adopt a risk-oriented subjectivity. Within this context the disciplinary power of risk discourse is obscured by liberal accounts of 'choice'; and to this extent liberalism is viewed in this analysis as an auxiliary form of 'governmental rationality' (Weir, 1996). As Lupton suggests, "The rhetoric of public health discourse is such that the individual is unaware that the discourse is disciplinary; health is deemed a universal right, a fundamental good, and therefore measures taken to protect one's health must necessarily be the concern and goal of each individual” (2003:35). 
This view is consistent with broader Foucauldian-informed analyses of the operation of biomedicine. Within this perspective, the protocol of informed consent for prenatal screening, in effectively responsibilising individual women for their own risk management, has been theorised as among the range of activities and processes through which the institution of public health maintains its disciplinary power over individuals (Lupton, 1995, Weir, 1996). Thus as Helen contends, the coexistence of routine screening and protocols of informed consent in contemporary prenatal care generates a paradox according to which individual women are "at once free and compelled to give their informed consent" (2002:119).

The personal ramifications (in terms of clinical ethics) of a 'risk subjectivity' for individual consent have received little attention, and as suggested earlier in the chapter it would seem methodologically difficult to evaluate the extent to which women's experience and decision-making is broadly shaped by discourses of risk. However, as chapters three and four considered, the accounts of those who position themselves outside the parameters of this frame offer rich insights into the way risk is individually experienced. Such accounts provide a platform from which to theorise the broader significance of risk for individual consent. Within this study, the accounts of two participants in particular, Wilma and Rona, usefully highlight the significance for women of a risk orientation in the provision of prenatal care, in particular drawing attention to the substantive difference between notions of 'choice' and 'consent'. Both women explicitly rejected a designation of their pregnancies as risky, instead drawing on embodied knowledge, past experience and personally valued ideologies as the informational bases of their decision-making. Wilma (undergoing a pregnancy in her mid-forties) rejected the categorisation of her pregnancy as 'at risk'. Rather she 
viewed her age and experience from previous pregnancies as standing her in good stead for the birth of her next child. As she remarked:

To me it wasn't really old, and I just felt that I was fitter and more able to carry a baby than some of the younger women anyway. So to me the age wasn't significant.

Wilma described the suggestion that she should undergo amniocentesis as an "unwelcome intrusion", and stated that she felt her wish to decline this form of screening was not viewed as legitimate; for while her midwife accepted her decision to decline, the 'paper trail' which attended this choice clearly signalled that this was not in fact a sanctioned pathway:

She had to fill out the details ... which indicated to me that there was an expectation from the medical profession that she needed to protect herself. You know, I mean she wouldn't have filled out all of that stuff and put it all in the notes if there was an acceptance on the medical profession side that "no" from me was enough.

In highlighting the bureaucratic response to patient decisions which fall outside the established parameters of consent, this account resonates with Corrigan's concern that institutional requirements for consent may reduce individuals to "objects of the bureaucratic machinery" (2003:786).Wilma's cynicism about her caregiver's obligation to essentially 'go through the motions' of informed consent arguably speaks of an ethos of health care which focuses less on preserving authentic autonomy than the mechanistic fulfilment of procedural criteria.

Rona similarly resisted the view of pregnancy as a time of risk, and she described her choice to decline screening in terms of the embodiment of broader political ideals. For her, choosing not to engage with screening expressed a wish to position her entire 
pregnancy experience outside the purview of the 'medical model' of childbirth. In deliberately choosing a midwife with a 'hands-off' practice style, Rona described how the need to formally discuss screening was effectively pre-empted by informing her midwife early in their relationship of her personal belief that screening was unnecessary. While her midwife nonetheless made the option of screening available, Rona's account suggests that she did not feel any sense of obligation to make a particular decision:

Oh well it was offered, and I was given a form and told, you know, if you want to go and have it done, go and have it done. But I just didn't, and there was absolutely no pressure at all. And I said "are there any issues if I don't have screening?" and the midwife said "no".

However, as the following account suggests, she was nonetheless unable to entirely deflect broader social criticism of her choice to decline screening as risky and irresponsible:

I got annoyed when someone suggested I was being irresponsible by not screening ... it was my sister-in-law. And it wasn't to me, it was to a group of other people saying "oh don't you think she's being irresponsible?" And that annoyed me because I thought, well I just thought actually no, what I'm doing is making a decision that is actually really accepting and really responsible, as I see it. And is not putting anybody's health at risk, and quite the reverse.

This perhaps suggests the extent of social expectations that individuals will and should consent to available medical procedures, indicating the normative ascendancy of popular notions of medical beneficence and authority over liberal accounts of informed consent as a vehicle of individual choice. Similarly, Wilma's comments suggest that in the popular view, compliance with (i.e. consenting to) medical orthodoxy may be valued more highly than the principle of autonomous decisionmaking per se: 
I know that it has increased in society, the pressure for some of those tests has just become much more standard, much more expected. And less tolerance of people saying no to it.

These accounts of the decision-making process suggest that contrary to contemporary clinical ethics' insistence on the provision of information as a requirement of autonomy, some individuals do not wish to 'be informed' other than in terms of their own pre-existing understandings. As Corrigan (2003) suggests, in some cases the requirement for consent is experienced as unwelcome or even burdensome.

Press \& Browner (1994) have theorised women's generalised acceptance of screening in terms of an inchoate strategy of avoidance of the burden of moral decision-making. In this view, collusion with the medical 'fiction' of screening as a tool of reassurance rather than diagnosis is suggested as a welcome egress from expectations of individual moral agency. In contrast, it is interesting to consider the choice to decline screening with respect to notions of patient agency as it is conceived within contemporary models of health care. Wilma and Rona's accounts suggest that this decision has les to do with a wish to avoid moral decision-making, than the fact that their respective moral terms of reference do not fit with those prescribed by a riskbased rationale for screening: in recognition of this, they simply choose not to engage. Such observations draw attention to the fact that within the context of a standardised medical/technical procedure, processes of consent necessarily draw individuals into engagement with epistemologies which they may reject; this raises the question of a need to reconsider clinical assumptions about reasons why some women do not 
participate in screening programmes, a phenomenon which as noted previously is usually attributed in clinical literature either to lack of information or knowledge.

As suggested earlier, current conceptualisations of informed consent and 'patient rights' assume that individuals wish to know as much as possible. However, these experiential accounts throw into question the presumption that a lack of information is the most significant obstacle to informed decision-making, and signal the need for a consideration of other threats to personal autonomy, such as those generated by the imperatives of a risk-based model of healthcare. As chapters three and four suggest, the experiences of women who resist the norms of screening show that some pregnant women place a high premium on being valued as the primary agents of their care, and that as Rudolfsdottir (2000) has argued, experiencing this agency as under threat has the potential to transform a positive pregnancy experience into a negative one. For participants such as Anna, who felt less able than Rona or Wilma to deflect an unwelcome designation of risk, the consequences of a diminished sense of agency were clearly evident in her sense of a loss of control over the direction of her care: "I felt we were caught in this trap, you know? I couldn't get off it. We were in the system and we couldn't get off it".

Garcia et al (2008a:753) have argued that "an important ethical condition for the introduction of prenatal testing is that women should decide according to their own values and be able to come to terms with their decision". In accordance with this view, it seems important to consider how the choice to 'opt out' of normative screening pathways might be formally recognised both within clinical ethics, and more broadly within mainstream screening discourse. As this discussion suggests, 
'risk', as the conceptual frame which characterises the constitution of prenatal screening as a 'public health activity', does not sit comfortably with the liberal conception of screening as a mechanism of individual choice. This fits Shildrick's contention that within contemporary health discourse more generally, notions of 'choice' and 'consent' tend to be conflated in a way which obscures the practical constraints on individual autonomy (1997).

\section{Significance of the practitioner-patient relationship for informed consent}

Press \& Browners' research (1997) has indicated that practitioners play a fundamental role in shaping individual women's understanding of prenatal screening, and that in fact institutional endorsement is the most significant determinant of the 'uptake' of this technology. Such studies suggest that prior to the first prenatal visit, at which prenatal screening is generally discussed, a significant number of pregnant women have not had access to specific information relating to available techniques sufficient to enable them to make an informed choice. Therefore, as the primary representational agents of screening practices, individual practitioners can be seen to play a pivotal role in mediating women's decision-making. Milligan has conceived of the ideal encounter between practitioner and pregnant woman as an opportunity for both parties to engage in "meaningful ethical discussion" about prenatal screening (2004:11).

However as I will suggest, a number of factors constrain the opportunity for ethically meaningful dialogue and decision-making within this context.

Singer et al (2001) have argued that the doctor-patient relationship is, and should be, at the heart of clinical ethics. However they note that in spite of an increasing emphasis on clinical ethics in medical training, this relational context persists as a deeply problematic site and one which far from guarantees good outcomes for 
patients. Evidence discussed elsewhere in this thesis raises a number of questions about the satisfactoriness of reliance on individual practitioners to facilitate women's informed consent for screening. As Corrigan (2003) has suggested, the significance of the doctor-patient dynamic for informed consent appears to be under-recognised in clinical ethics.

Shildrick (1997) contends that contemporary models of informed consent assume an equitable 'partnership' relation between practitioner and patient within which decision-making (and ultimately consent) is conceptualised as mutually achieved. However, this assumption is problematic. As Corrigan (2003) suggests, the practitioner-patient dynamic is shaped by pre-existing cultural norms, including popular assumptions that medical practitioners will always act in patients' best interests, and that patients themselves 'should' defer to the authoritativeness of medical expertise (Press \& Browner, 1996). ${ }^{129}$ One potential consequence may be patients' assumption that they need not themselves actively deliberate over their own health decisions, as their clinicians will advise them of the best course of action. With respect to prenatal screening specifically, this concurs with Press \& Browner's findings $(1994,1997)$ that for some women this approach may be welcomed as a 'way out' of the individual burden of moral agency. In this view, women's consent to screening as a routine aspect of pregnancy care is seen as instrumental in a wish to avoid having to confront the uncomfortable (for some women) ethical dilemmas associated with screening (chiefly, the notion of selective termination as eugenic). Whether or not women benefit from the capacity to retreat in this way from making

\footnotetext{
${ }^{129}$ With respect to midwifery-led care in the New Zealand context, a similar critique has been directed at the 'partnership' model, which similarly assumes an equal power dynamic between midwives and individual pregnant women in their care. See for example, Freeman \& Timperley (2004).
} 
difficult ethical decisions, such expectations of clinicians' role clearly problematise the assumption of autonomy which underpins current models of consent.

A further problem relates to the quality of information provided by practitioners. As discussed previously, the inadequate and inconsistent provision of information to pregnant women by individual practitioners is increasingly recognised as an endemic problem in the provision of screening services. Graham et al (2000) have argued that the practitioner's role in the provision of information to patients constitutes one of the most serious obstacles to the achievement of informed choice for prenatal screening. Certainly a concern over insufficient provision of information by practitioners is a consistent theme in the experiences discussed in this thesis, with a majority of participants indicating that this one to one encounter was seen both as a core 'problem' and potential solution to concerns over the achievement of informed consent as a practical ideal. However, the view that provision of information per se fulfils a commitment to informed consent has drawn critique. As Lopez (2004) notes, the belief that practitioners can, through the implementation of various procedural requirements, 'confer' autonomy on their patients is problematised by broader social scientific critiques of contemporary processes of consent; in particular of the assumption that it is possible to abstract individual decision-making from the broader social context.

It therefore seems important to consider the context as well as the content of individual consent as a matter of ethical concern. Shildrick (1997) argues that while formal definitions of consent emphasise the conditional nature of consent, that is, the fact that consent is dependent on criteria relating to the context and status of the agent 
(for example the requirement that an individual must not be coerced, and must have sufficient information), within the day to day clinical setting little analysis is actually brought to bear on the significance of the context within which individual consents are sought. In this respect she argues that contemporary bioethical conceptualisations of consent reflect and consolidate commonsense understandings of 'consent' as simply an 'agreement to'. However, as Kohut et al (2002) suggest, the notion of informed consent as "a discrete event of obtaining legal approval from a patient for a procedure" does not reflect either contemporary expectations of patient autonomy, or the processual nature of decision-making which in reality is characterised by choices involving personal values.

Scully et al (2007) have argued that current models of informed consent presume that individuals necessarily understand the information they are given. This is an observation similarly made by Williams et al (2005) who suggest that the use of technical language in the transmission of information may itself be a barrier to autonomy, for example the use of statistical ratios to communicate the risk of Down syndrome to women receiving NTS results. As noted in chapter four, current models of consent also may not adequately recognise individuals' need for sufficient time in which to reach personally acceptable decisions, an argument well-illustrated in this study within Ruby's experience (see below). With the implementation of firsttrimester screening, the expectation that pregnant women make their screening choices within the context of a single prenatal visit (i.e. before the clinically appropriate time frame has passed for the NTS) raises further questions about the 
ethics of this approach. ${ }^{130}$ Furthermore, as recently conducted practitioner surveys in New Zealand suggest, a considerable number of maternity practitioners themselves lack accurate, current knowledge of available screening techniques and their appropriate use (Stone \& Austen, 2006). With respect to the practice of prenatal ultrasound specifically, the phenomenon of 'false-negative' results is recognised in clinical literature as significantly deriving from an endemic level of practitioner error, ${ }^{131}$ a confounding variable in screening results about which women rarely appear to be advised in advance by their caregivers.

The question of directiveness also potentially complicates practitioners' role in advising and counselling women about screening options. As noted in the work of Farsides et al (2004), a degree of moral ambivalence 'haunts' practitioners in their provision of prenatal screening services. Again, the visual nature of ultrasound imaging may have an underacknowledged significance in shaping clinicians' practice; as will be discussed in chapter seven, there is evidence to suggest that fetal imaging may compound practitioner's existing moral views on matters such as selective termination, potentially influencing the tenor of information conveyed to women during scans. Similarly, struggles to integrate conflicting professional perspectives on questions such as the place of routine medical intervention, and the validity of notions such as 'informed refusal', also raise dilemmas for individual practitioners, potentially influencing the manner and content of counselling around screening within their practice (Williams et al, 2002c, Irving, 2004). For these reasons, exclusive reliance on individual practitioners to facilitate informed consent would seem very

\footnotetext{
${ }^{130}$ Pregnant women often do not have their initial prenatal visit until several menstrual periods have been missed. Given that NT scans should ideally occur between 9-11 weeks pregnancy, this places a considerable time pressure on decisions around screening to occur during a prenatal visit, in order that a referral for a scan appointment can be arranged within the necessary timeframe.

${ }^{131}$ These findings are discussed further in chapter six.
} 
vulnerable to the vagaries of personal viewpoint. Another dimension of potential practitioner directiveness which is specific to prenatal ultrasound is the fact that unlike several other screening methods, such as amniocentesis and CVS, prenatal scans do not currently require a written consent from pregnant women. This reliance on verbal (i.e. less obviously 'formal') consent may arguably reinforce popular perceptions of ultrasound as a screening method with 'less serious' implications than other techniques. Given these potential limitations of the conditions for informed consent, the extent to which pregnant women are currently vulnerable to the representational 'tone' of specific encounters with their caregivers may need more thought.

However, as Corrigan (2003) has suggested, the roots of the problem of directiveness may extend beyond the practice of individual clinicians. As she observes, lay expectations around medical beneficence ${ }^{132}$ may predispose patients to interpret a practitioner's attempt simply to raise the issue of consent as a guidance to give consent to the procedure being discussed. Furthermore, she argues that for a considerable number of patients, agreement to give consent may be significantly motivated by a wish to comply with what is perceived to be an actual request by their caregiver. ${ }^{133}$ Practitioners themselves may be ambivalent about the practical achievement of 'informed informed' consent. Recent UK studies investigating practitioners' attitudes towards existing prenatal screening services indicates that nondirectiveness is generally regarded by this group as an impractical (and sometimes undesirable) ideal (Williams et al 2002a, 2002b, 2002c). As this and similar research

\footnotetext{
${ }^{132}$ That is, the notion that clinicians will always act in the best interests of the patient.

${ }^{133}$ To support this claim Corrigan cites a study by Bevan et al (1993) on patients' attitudes towards participation in clinical trails, which notes that 38 per cent of consenting participants stated that their agreement to consent was motivated in this way.
} 
suggests, many practitioners believe that the constraints of time and resourcing which characterise many contemporary health care settings present virtually intractable obstacles to the "complex interactional work" (Pilnick et al, 2004:83) necessary to meaningfully communicate complex information to patients. This difficulty faced by practitioners is further illustrated by recent research on patient views of what constitutes an informed consent. El-Wakeel et al (2006) note an expectation that practitioners explain all possible clinical outcomes, a requirement which it is suggested is practically impossible to fulfil. Philosophical tensions between different models of care may further complicate the role of practitioners in facilitating patients' informed consent (Williams et al, 2002b). ${ }^{134}$

\section{Ruby's story: a case study of the problematic of informed consent for prenatal screening}

As chapters three and four observed, concern about the sufficiency of information and the inadequacy of pre-test counselling were themes common to many participants' accounts. A number of these women reported feeling that practitioners' conduct did not reflect a sensitivity to the potential personal significance of screening, and sometimes conveyed a sense of inappropriate casualness. This sentiment was characterised by Naomi as "treating a heavy thing as a light thing".

When viewed as a case study of 'informed consent' the following account highlights the lived impact of insufficient clinical attention to the ethical requirements of informed consent for prenatal screening. The account also personalises the issue of the clinical reliability of NTS, highlighting the manner in which the procedure, when

\footnotetext{
${ }^{134}$ The existence and implications of such tensions within the New Zealand context are discussed in chapter six.
} 
used as a stand-alone method, may trigger a sequence of unnecessary (and clinically risky) interventions. While it is not claimed that Ruby's account should be considered as representative of participants' experience, it does clearly reiterate a number of the experiential themes discussed in chapter three. As Stake has suggested, the value of examining specific cases lies in their capacity for the "extension of experience" (2000:449). In keeping with this view, the following discussion of Ruby's specific experience is included to add contextual depth to the investigation in this thesis of prenatal screening as a privatised, invisible experience.

A 31-year-old first time mother in 2005, Ruby had discovered she was pregnant after going to hospital with unexplained vaginal bleeding. At this point she was offered NTS, being advised that this was a 'standard test' conducted at this duration of pregnancy. She was given no information about the nature or possible outcome of the test (including the fact that the test was to detect Down syndrome). Immediately following the scan, Ruby was advised that she had a 1:12 (i.e. highly significant) chance of having a baby with Down syndrome. She was then discharged from hospital, and advised to make a follow-up appointment with her general practitioner (GP). Ruby made this appointment, during which her GP said "I have taken the liberty of booking you in for a termination". Ruby recounted that she was taken aback that not only had she received no information about Down syndrome and the options available to her (including support available for proceeding with the pregnancy), but also that a termination had been booked without her consent. At this point she questioned whether there might be some other test available to double-check her results. The GP then cancelled the termination and booked her for a CVS, an invasive test similar to amniocentesis which provides a definitive genetic diagnosis of Down 
syndrome. ${ }^{135}$ As the CVS was about to be performed, the obstetrician questioned the accuracy of Ruby's previous NTS result, suggesting that what might have appeared to be a thickening of the fetal neck (i.e. an indication of Down syndrome) was perhaps simply the edge of the placenta. She suggested that Ruby wait three weeks by which time the pregnancy would be sufficiently advanced for an amniocentesis to be performed, which she did, by this time being 15 weeks' pregnant and highly anxious and uncertain about the status of the pregnancy. Ruby described this period of waiting as a time when she attempted to "shut off" any sense of emotional or bodily connectedness with the pregnancy. As she describes, this sense of provisionality and disconnection was not easily maintained, and came at a personal and social cost - as the pregnancy was concealed from family and friends, Ruby was deprived of a source of emotional support and, potentially, information.

Ruby's account of the amniocentesis exemplifies the tension between the social and medical uses of ultrasound:

The radiologists $[\mathrm{sic}]$ at the hospital were just saying "oh look at it, isn't it cute, isn't it sweet", as they looked at this image. And I was just trying not to imagine it being a baby at all, I was just trying to ... they were saying "oh look at its little hands" and I was thinking yeah, so those little hands are going to die really soon, aren't they.

After a further three-week wait for the results of the amniocentesis, ${ }^{136}$ during which time Ruby received no information about the point at which termination of pregnancy would no longer be an available option, Ruby was informed by her GP over the

135 "Chorionic Villus Sampling". During CVS a needle is inserted through the cervix to obtain a sample of placental tissue, and an ultrasound scan is performed concurrently to check the position of the needle in relation to the fetus. Both amniocentesis and CVS carry an approximate $1 \%$ risk of miscarriage (Nicolaides et al, 2005).

${ }^{136} \mathrm{~A}$ two week waiting period is typical before a result is available from amniocentesis. In some instances results may take longer due to such technical difficulties as the contamination of the sample of amniotic fluid with blood. This was what had occurred in Ruby's case. 
telephone that her result was chromosomally normal, and advised to find a midwife. She went on to give birth to a healthy, chromosomally normal baby girl several months later.

In her encounter with prenatal screening, Ruby appears to have been deprived of an opportunity to experience her pregnancy as anything other than provisional, and certainly not as 'normal'. As her account suggests, this sense of provisionality had lasting consequences, enduring well beyond the point at which the 'normal' status of her pregnancy was medically confirmed:

For the first 25 weeks ... I didn't ever think the word 'baby', I talked 'fetus', because you know, that's a thing. And even during the labour, I think when I went into labour and the midwife asked me what sort of nappies I'd brought in or wanted to use, disposable or cloth nappies, and I sort of went "oh my god, that's right, there's a baby in there".

It is significant to note that subsequent to her normal result, Ruby was not offered any form of clinical follow-up or counselling which might have assisted her in this readjustment. It was a matter of considerable concern for Ruby that while three forms of screening had been either performed, (or, in the case of CVS, nearly performed) NTS, CVS and amniocentesis - at none of these junctures did she feel she had received information or counselling sufficient to enable her to give what she regarded as an informed consent. Where written consent had been obtained (i.e. a signature authorising the amniocentesis), she explained that this had felt like a procedural formality rather than an opportunity to deliberate:

Nobody ever spoke to me. I think what I found hardest was there was just no information given to me to base these decisions on. Like when I spoke to the obstetrician, I felt like I was making a decision in a vacuum. I was expected to make these really important decisions without actually being aware of, you 
know, what the consequences were, what things really meant ... I didn't actually have anyone to turn to.

In the absence of information about alternative paths of action, Ruby was left to do her own research, resorting to internet searches and ultimately, as she put it, "negotiating my own way through the hospital system".

The specifics of Ruby's experience suggest an endemic disregard for her rights of autonomy, evident primarily in the wholly inadequate provision of information and counselling as a platform for individual decision-making. This lack of regard can be seen to operate on a number of levels - in the professional shortcomings of individual practitioners, and perhaps also in terms of an institutionalised 'culture' of somewhat casual attitudes towards prenatal screening within medicine, as suggested above. In addition to the consequences of a lack of information, Ruby's account reveals an additional dimension of constraint on her ability to make 'free and unfettered choices' as conceived within contemporary models of consent. As will be further discussed in chapter seven, the discursive 'baggage' of ultrasound's visuality problematises notions of 'choice' and 'consent' with regard to screening, due to the entwinement of 'social' and 'medical' meanings ascribed to fetal imaging. In Ruby's case, the sonographer's insistent personalisation of the fetus reflects a 'social' conceptualisation which is directly at odds with the primary medical rationale of the procedure, which was being performed specifically to confirm a previous diagnosis of fetal abnormality. To this extent, Ruby's experience succinctly evinces and personalises Taylor's conception of the 'prenatal paradox' of fetal ultrasound (1998), highlighting the perversity of the coalescence of expectations of provisionality and 
unconditionality which are attached to the use of prenatal ultrasound as a medical project.

It could be argued that in constituting her fetus as a baby, and thereby privileging a 'social' understanding of the ultrasound image, the sonographer eroded the availability and legitimacy of alternative discourses which Ruby might have utilised in her decision-making. The attribution of the characteristics of an already born baby, in its 'cuteness' and 'sweetness', subtly but irrevocably shifts the frame of reference against which Ruby's subsequent decision-making then had to take place.

While in this case the sonographer may not have failed to meet either technical standards or indeed any specific code of ethical conduct set out by her profession, her practice nonetheless seems unethical, and this perhaps speaks of the broader discursive tensions which underpin contemporary screening practice. That the ethical stakes of the ultrasound scan as a 'representational moment' are so high surely calls for a re-evaluation of what constitute appropriate discursive parameters within the practice of sonography. As Garcia et al note, "the moral significance of prenatal testing is inseparably bound with the social context in which it is practiced" (2008b:115). Appearing to draw selectively on multiple registers of discourse within their practice, the pivotal role sonographers may play in determining the discursive character of individual women's scan experiences is arguably yet to be fully recognised as a matter of clinical ethics. ${ }^{137}$

Ruby's case illustrates Rudolfsdottir's findings that within the context of contemporary prenatal care, practitioners do not always make provision for consent,

${ }^{137}$ This will be considered in chapter seven. 
and the pregnant woman is "not always treated as a fully responsible agent"

(2000:345). In highlighting the manner in which individual practitioners sometimes appear to 'default' to paternalistic models of consent, her experience suggests how tenuous reliance on the practitioner/patient relationship may be as the supposed foundation of contemporary clinical ethics. Thus it seems reasonable to suppose that even where contemporary models of consent are followed, as Corrigan suggests "it cannot simply be assumed that the imposition of the informed consent process will necessarily bring about an equitable doctor-patient relationship where patients make active choices" (2003:788).

\section{Conclusion}

This chapter has identified current practice in prenatal screening as a problem for and of clinical ethics. It has been argued that the constitution of prenatal screening as a public health concern has significant and largely unacknowledged consequences for individual agency, casting doubt on the ethical 'robustness' of reliance on protocols such as informed consent. Within this discussion and throughout preceding chapters, participant accounts of decision-making around prenatal screening highlight the individual significance of a generalised risk orientation in prenatal care, and signal the need for a reconsideration of ethical practice. Chapter eight will consider ways in which such concerns might practically be addressed, focussing specifically on the New Zealand context as a site with potential for a re-drawing of ethical practice in prenatal screening. That some urgency should be mustered in the attempt to attend to current practice within New Zealand is resoundingly evident in Ruby's account, which concludes this chapter: 
My feelings towards pregnancy support in New Zealand have changed as a result of this experience. I'd been living in Asia for awhile, and in Asia I expected not to be informed about things. And for doctors to make decisions and not tell you why they'd made decisions, and to just sort of be chugged through the process without, you know, being a patient but without being a partner in decision-making. I didn't expect that in New Zealand, but that's how I felt. So I was quite surprised about that, my sense of superiority coming from this, you know, "advanced" society (was) quashed fairly quickly. 


\section{Chapter Six}

\section{The New Zealand experience: the representational politics of prenatal screening as a contemporary public health concern:}

Recent developments make New Zealand a fascinating sociological case study of the uneasy fit between medical and social understandings of prenatal screening as a project of health. This chapter explores the discursive/rhetorical 'management' of these tensions within recent public health discourse. First, I will outline the provision of screening within the New Zealand maternity care system, and describe recent developments which have seen the issue draw unprecedented attention in terms of policy development and processes of public relations/engagement. I will then consider a number of structural factors within the New Zealand maternity care system which have historically delimited women's screening choices. This provides necessary background information for the following discussion and establishes the cultural context for participants' accounts within previous chapters. Finally, I will consider how prenatal screening has recently been constituted as a health concern within the public health domain in New Zealand, drawing on selected informational material produced for public consumption. This section explores the representational politics of recent changes to prenatal screening practices in New Zealand, raising the question of whether present policy and practice in fact constitutes a national screening programme for Down syndrome, even though not officially described as such.

\section{Prenatal screening in New Zealand: A brief history}

There is limited literature relating to prenatal screening within New Zealand specifically, and certainly to date the topic has drawn little interest as a matter of 
social scientific inquiry within this country. ${ }^{138}$ What literature is available is almost exclusively produced within the medical/epidemiological domain. As might be expected, this literature typically focuses on the pragmatics of screening as a medical project, and it is evident that prenatal screening for conditions such as Down syndrome is understood as a 'fait accompli' of best practice in contemporary pregnancy care. While the ethical dilemmas and potential personal costs associated with screening may be acknowledged, ${ }^{139}$ the primary concern within such material is the improved efficiency of screening practices. A very limited amount of midwifery literature is also available evaluating screening practice within New Zealand. ${ }^{140}$

Within the New Zealand maternity care system, prenatal screening services are provided to pregnant women by midwives, obstetricians, and in some areas, general practitioners. Midwives oversee $70 \%$ of maternity care in New Zealand (NZCOM, 2009), and have been able to practice autonomously since $1990 .{ }^{141}$ Within the New Zealand maternity system, a 'primary care' model is endorsed, with state-funded access to prenatal, birth and postnatal care available to women registering with either a midwife or general practitioners as their 'lead maternity carer' (LMC). ${ }^{142}$ It should be noted that the model of maternity care which operates in New Zealand is in clear contrast to many countries, including Australia as New Zealand's closest neighbour, where maternity care is primarily obstetrician-led, and as Zadoroznyj (2000) has noted, obstetrics bodies such as RANZCOG are by and large not prepared to endorse

\footnotetext{
${ }^{138}$ Oakley's' 1997 MA thesis (Health Sciences) “ Women's subjective experience of the ultrasound examination during pregnancy" is the only available example of qualitative research in this area found to have been undertaken in New Zealand to date.

${ }^{139}$ See for example, Stone (2005).

${ }^{140}$ See for example Irving (2004), and Donley (1990).

${ }^{141}$ In accordance with the 1990 amendment of the 1977 Nurses' Act.

${ }^{142}$ Women requiring referral for specialist obstetric care for medical reasons are also fully funded. However, the cost of elective use of specialist obstetric care is carried privately.
} 
a primary-care model of maternity services ${ }^{143}$ However, Australian medical/public health literature on prenatal screening mirrors the concerns around inconsistent practices and variable access to screening recently voiced within New Zealand, and reflects a similar sense of momentum towards the implementation of a formalised national screening programme. ${ }^{144}$

As in many other countries, prenatal screening in New Zealand has historically been primarily directed towards the detection of Down syndrome. The National Screening Unit (NSU, 2007b) notes that approximately 70 children with Down syndrome are born in New Zealand annually, with approximately 60 pregnancies per annum terminated following a positive test for Down syndrome in New Zealand (Toi te Taiao/Bioethics Council, 2007). At present, New Zealand does not have an official national screening programme for Down syndrome. However, within recent years, calls from within the obstetric and public health community for an urgent reappraisal of current practice ${ }^{145}$ have led to a process of ministerial review on the provision of prenatal screening services. Revelations of inconsistent and unsafe practices have seen sustained agitation within the public health domain for reform of current practice, with the implementation of a formalised national Down syndrome screening programme proposed by some health officials as a necessary 'next step'.

\footnotetext{
${ }^{143}$ The Australian Medical Association (AMA) recently expressed the view that doctors are more suitable than midwives to care for pregnant women, even those experiencing 'low-risk' pregnancies, the organization explicitly drawing a link between midwifery-led care and increased rates of perinatal mortality (AMA, 2005).

${ }^{144}$ See for example O'Leary et al (2006) "Regional variations in prenatal screening across Australia: stepping towards a national policy framework".

${ }^{145}$ See especially Stone (2005) "Prenatal testing for aneuploidy in New Zealand: time for action", and Chang (2006) "Antenatal screening for Down syndrome in New Zealand: time for a national screening policy?" See also Irving (2004) "Nuchal translucency screening for Down's syndrome: the midwife's role" for a midwifery-oriented critique.
} 


\section{Current practice in New Zealand}

At present, Nuchal Translucency Scanning (hereafter NTS) is the most widely-used method of screening for Down syndrome in New Zealand, a method which occurs within the first trimester of pregnancy (Stone \& Austen, 2006). However, this method of early pregnancy screening has only been available in New Zealand since the early 1990s and historically screening for Down syndrome in New Zealand has occurred during the second trimester of pregnancy. An age-based strategy has been the predominant approach, which appears to have been directed by protocols produced within the obstetric community (i.e. the Royal Australian and New Zealand College of Obstetricians and Gynaecologists or RANZCOG) that all pregnant women older than 35 years of age be offered invasive testing (either amniocentesis or CVS). In addition, as Chang (2006) notes, practitioners have tended to rely on fetal growth and anatomy scans (routinely offered at 18-20 weeks pregnancy) as an opportunity to visually identify possible signs of conditions such as Down syndrome. As this scan, which is not officially intended as a method of screening, has historically been made available to the vast majority of pregnant women in New Zealand irrespective of age, it seems a reasonable assumption that for the majority of New Zealand women pregnant prior to the early 1990s, when NTS became available, the 18-20 week anatomy scan is likely to have been the sole encounter, within the context of their pregnancy care, with the notion of 'prenatal screening' and its associated dilemmas. ${ }^{146}$ As Stone and Austen (2006) have commented, this "opportunistic" use of ultrasound as a means of detecting fetal abnormality is somewhat unique to New Zealand's screening history; while many overseas countries have historically relied on maternal blood screening

\footnotetext{
146 This was certainly true of women within the participant sample who had received pregnancy care prior to the early 1990 s.
} 
(second trimester) as the basis of screening strategies, ${ }^{147}$ within New Zealand ultrasound has essentially provided the clinical platform from which current screening practice has evolved (2006). As they argue, within New Zealand "modern screening began by using ultrasound techniques and has gradually been widely offered but not in the context of a coordinated screening programme" (2006:5). While maternal blood testing has been available in a limited capacity in New Zealand in recent years, this has not historically been funded by the Ministry of Health, and women seeking this additional screening method have been required to pay $\$ 75 .{ }^{148}$ Stone $(2005)$ has suggested that the very low uptake of this test in New Zealand may be explained in part by a perceived lack of clinical endorsement due to this historic lack of funding. In these respects, it might be suggested that New Zealand women have historically been able to access fewer clinical options or 'choices' around screening than women in other Western countries, including Australia. ${ }^{149}$

In 2004 The National Screening Unit (NSU) (a body working under the auspices of the New Zealand Ministry of Health) held a workshop on prenatal screening for Down syndrome, noting the widely held view among clinicians that current practices were unsatisfactorily co-ordinated. Subsequently professor of Obstetrics Peter Stone was commissioned to undertake a national evaluation of current screening practices and assess the viability of a national screening programme for Down syndrome in New Zealand. The subsequent report ${ }^{150}$ provided the first-ever overview of screening

\footnotetext{
${ }^{147}$ Chang (2006) notes that in Europe and the USA second trimester maternal blood tests have been the primary approach to prenatal screening since the 1980 s.

${ }^{148}$ However it should be noted that from 2008 (subsequent to the 2007 review of screening for Down syndrome in New Zealand, see below), a second trimester maternal blood test has been made available free of charge as a temporary adjunct to NTS.

${ }^{149}$ Lawrence et al (2003) note that maternal blood screening has been widely used in Australia since the early 1990s. While NTS is used less often in Australia than in New Zealand, it is nonetheless offered in over 50\% of Australian hospitals providing core prenatal care services (Cheffins et al (2000). ${ }^{150}$ Stone and Austen (2006), Report to the National Screening Unit: assessment of antenatal screening for Down syndrome in New Zealand.
} 
practices in New Zealand, and included data from a survey of all practitioners involved in the provision of screening to pregnant women (obstetricians, midwives, GPs and sonographers). The report signalled a need for urgent reform, with the authors concluding that "the current situation of ad hoc screening, increasing NT scans and amnio rates is not leading to efficient detection of Down syndrome fetuses" (Stone \& Austen, 2006:38). Key concerns related to the current reliance on NTS as the primary method of detecting Down syndrome in New Zealand, ${ }^{151}$ and the continuing use of an outdated protocol of age-based screening by many practitioners. The authors noted that as a result of these factors, many New Zealand women were being unnecessarily referred for amniocentesis, with subsequent rates of miscarriage of normal fetuses outstripping the rate of detection of fetuses with Down syndrome: "best estimates would suggest that more normal pregnancies may be adversely affected by amniocentesis than the numbers of Down syndrome fetuses detected" (2006:5). ${ }^{152}$ Additional concerns related to poor levels of practitioner knowledge around appropriate use of screening, and insufficient training and regulation of sonographers providing NTS, leading to high rates of incorrect diagnoses. The report concluded that current practice in New Zealand was inconsistent, and not in line with internationally recognised 'best practice'.

In response to these findings, the NSU established the Antenatal Down Syndrome Screening Advisory Group in 2006, ${ }^{153}$ with the directive to "examine interim measures to improve access, safety and quality of the existing screening and to examine the need for and acceptability of a nationally organised Down syndrome

\footnotetext{
${ }^{151}$ As noted in chapter two, this method is associated with a high rate of "false positive" results when used as a 'stand alone' screening method (i.e. without an auxiliary maternal blood test).

${ }^{152}$ Stone (2005) notes that only $1.2 \%$ of amniocenteses performed produce a positive result for Down syndrome, a fact presented as evidence of the primary flaw of reliance on age-based screening.

${ }^{153}$ Membership of the working group included representatives of the obstetric, midwifery and general practice communities, geneticists, health policy analysts and representatives from the disability sector.
} 
screening programme" (NSU, 2007a:v). The Advisory Group produced a report for the Ministry of Health entitled "Antenatal Down Syndrome Screening in New Zealand 2007". The key finding of the report was that "the current practice of screening using only NT and/or maternal age, without biochemical markers, is unsafe, inequitable and untenable, and should not continue" (2007a:54). After receiving the report, the then Minister of Health (Pete Hodgson) requested further advice on how a national screening programme might be implemented, pending a final decision over the reform of Down syndrome screening in 2008.

\section{Historic limitations on screening as a 'choice' for New Zealand women}

The conclusions of the review process focussed chiefly on clinical deficits and specific problems arising from these. While the likely psycho-social consequences of miscarriages following amniocenteses are recognised, ${ }^{154}$ the broader impact of historic screening practices within New Zealand on pregnant women's experience is not acknowledged. As participants' accounts within this study suggest, women's concerns about screening practices extend beyond the scope of technical shortcomings, tending to centre on matters directly related to personal experience, including issues of informed consent, and the dynamics of the practitioner-patient relationship as the context of their decision-making. In many cases a concern for the potential broader social impacts of prenatal screening was also evident. It is of note that neither of the two key reports on Down syndrome screening in New Zealand produced during the review process drew within their literature reviews on the large body of international qualitative research now available on women's experience of prenatal screening to guide their recommendations. Neither were public submissions invited during the course of the review in order to gauge consumer's attitudes and

\footnotetext{
${ }^{154}$ In particular within Stone and Austen's report (2006)
} 
preferences for screening practice. In this respect it could be argued that the review process has omitted the perspectives of those most intimately affected by clinical practice, thus failing to recognise the significance of subjective experience as a resource for directing ethical practice.

The review also fails to address the matter of historic Government inconsistency on the issue of Down syndrome screening in New Zealand, and the significance of this both for practitioners and individual women. It is of note that the NZ Ministry of Health, while not holding an official position on prenatal screening for Down syndrome, has nonetheless historically made funding available for ultrasound (NTS) screening to occur during each pregnancy. ${ }^{155}$ As the key government policy document outlining the provision of maternity services in New Zealand, Section 88 Primary Maternity Services Notice does not in fact list routine ultrasound screening within the standard schedule of services to be offered by maternity practitioners, providing a list of specific clinical indications for which the use of ultrasound is permitted. As Stone and Austen noted in 2006, the Ministry of Health had never issued clinical guidelines for the use of NTS in screening for Down syndrome, and regional health boards which employ individual maternity practitioners also did not produce clinical protocols.

In the absence of official guidance on undertaking prenatal screening, it appears to have been assumed that the various practitioner groups providing screening to pregnant women (midwives, general practitioners and obstetricians) will take responsibility for advising practitioners on best practice. However, it is far from clear

${ }^{155}$ Funding is allocated for NTS for each pregnant woman by the Maternity Benefits Department of the Ministry of Health. See Notice Pursuant to Section 88 of the New Zealand Public Health and Disability Act 2000 (Ministry of Health, 2002). 
how valuable this may have been in terms of ensuring New Zealand women access to consistent, evidence-based practice. Stone and Austen's 2006 survey of clinicians' screening practices revealed profound deficits in practitioners' knowledge of appropriate use of screening methods, and in particular, NTS. For example, $25 \%$ of practitioners surveyed did not correctly identify the appropriate gestation of pregnancy at which to perform this scan. In addition, $66.6 \%$ of practitioners performing NTS scans did not correctly identify the accepted risk range used to indicate a 'high-risk' screening result for Down syndrome (1:250-1:300). With respect to the provision of information about screening to pregnant women, a quarter of practitioners surveyed indicated that they did not discuss Down syndrome when advising women about prenatal screening, even though this is the primary condition being screened for.

Another interesting finding relates to practitioners' attitudes towards the general utility of prenatal screening, with only $59 \%$ of maternity practitioners surveyed holding the view that screening should be offered to all pregnant women. This finding was not investigated during the subsequent review; however this apparent tension between the views of a significant number of practitioners and the evident state endorsement and funding of population-based Down syndrome screening in New Zealand deserves closer scrutiny.

As Irving (2004) notes, the funding of Down syndrome screening in the absence of any Health Ministry directives has generated a number of dilemmas for practitioners in terms of juggling the imperatives of evidence-based practice, informed consent and consumer expectations. With respect specifically to midwives' practice, she has suggested that practitioners face a dilemma in reconciling the requirement to provide 
accurate and objective information to women about screening, with an awareness of the considerable shortcomings associated with available technology. The worry many pregnant women have about screening tests is also seen as generating a tension in midwives' role in providing reassurance and encouragement in the promotion of a positive view of pregnancy and birth. ${ }^{156}$

The significance of a 'multi-disciplinary' approach to maternity care for the provision of prenatal screening in New Zealand does not appear to have been accounted for in the Health Ministry's devolution of responsibility to practitioners. Ozturk (2008) notes a lack of professional collaboration between different practitioner groups in providing information and referrals for screening, suggesting that in some cases women miss out on necessary information and counselling prior to being referred for NTS. She notes that many women booking with a midwife have already been booked for NTS, often at the time they confirmed their pregnancy with their family doctor. The extent to which prenatal screening has been discussed or consent obtained is often not clear, as there is no protocol for the sharing of clinical notes between GPs and midwives; thus, for some women the decision to undergo screening appears to be clinically treated as a foregone conclusion, rather than a consensual process between pregnant women and the practitioner overseeing their pregnancy care. Such examples of a lack of clinical collegiality may have their origins in historic tensions between maternity practitioner groups within New Zealand. The significance of distinctions

\footnotetext{
${ }^{156}$ It appears however that a distinction should be drawn between the offer and endorsement of routine screening. An interesting finding of the practitioner survey was the evidence that midwives were the practitioner group most likely to offer screening routinely, with $69.8 \%$ of midwives surveyed offering screening to all women (in comparison to $65.8 \%$ of obstetricians). This would appear to contradict widespread assumptions of midwifery as a profession necessarily inured to a 'low-tech' philosophy. General practitioners were noted as the group least likely to offer screening to all women. Such findings may suggest professional variance in commitment to processes of informed consent within clinical practice, a hypothesis which falls outside the scope of this thesis but would be interesting to pursue.
} 
between midwifery practice and the medical model of care for the general provision of maternity services in New Zealand is acknowledged within government documents. For example, the 1999 review of maternity services by the National Health Committee notes:

There are two distinct views of the role of the clinician in childbirth with a wide spectrum of opinion between. Both midwives and medical practitioners place a high premium on the safety of mother and baby, but appear to view differently the extent to which childbirth should be treated as an inherently 'normal' event or a process with potential complications.

(National Health Committee, 1999:11)

However, with respect to the provision of prenatal care, it appears that such

differences are yet to be taken into account in terms of acknowledging potential differences there may be in the guidance given to practitioners by their respective professional bodies. There are in fact significant differences among these professional groups in the approaches taken to the provision of prenatal screening services. The New Zealand College of Midwives (NZCOM) is the advisory and representative body for midwives in New Zealand. The college does not make explicit any position on routine prenatal screening per se within its recommendations for practice. However, a consensus statement on ultrasound use produced by the College states that NZCOM does not support routine ultrasound screening in pregnancy, advising that "the selective use of ultrasound, with the woman's informed consent, is appropriate where there are specific individual indications" (NZCOM, 1995). Campbell (2008) notes that NZCOM endorses the position that pregnant women determine their own risk, and this appears to be the rationale for the provision of a list of reading materials in the College's Consensus Statement on Ultrasound as a resource available to women 
and midwives to evaluate ultrasound use (NZCOM, 1995). ${ }^{157}$ This view of the appropriate use of prenatal ultrasound appears to be in marked contrast to that of the Royal Australian College of Obstetricians and Gynaecologists (RANZCOG), which clearly advocates the use of ultrasound as a routine aspect of prenatal care, stating that "All women should be offered an obstetric ultrasound before 20 weeks' gestation" (2008:3). RANZCOG recommends the routine use of 'combined' firsttrimester screening (i.e. a combination of maternal blood tests and NTS) and secondtrimester maternal blood tests in all pregnancies as best practice in Down syndrome screening. Fetal development and growth scans at 18-20 weeks are recommended as an additional diagnostic opportunity. (RANZCOG, 2007).

The Royal New Zealand College of General Practitioners (RNZGP) has not historically issued clinical protocols for Down syndrome screening, and the offer of screening appears to be left to the discretion of individual practitioners. Stone \& Austen (2006) noted that general practitioners were the clinicians least likely to offer screening routinely. Thus a clear distinction is evident between the approaches taken to screening by the respective clinical bodies in New Zealand, a fact which would presumably see pregnant women receiving somewhat different evaluations of screening, dependent on which practitioner type they choose. It seems reasonable to suggest that the Health Ministry's devolution of responsibility for the clinical oversight of prenatal screening to a number of distinct professional bodies has to date failed to take in consideration the significance of the hybrid character of maternity care within New Zealand.

${ }^{157}$ See appendix 9 . 
The apparent reluctance of the Health Ministry to take an official position on Down syndrome screening is also evident in informational material produced by the Ministry of Health. It is interesting to note that while public information material is produced on other types of screening relating to maternity care (for example, HIV screening in pregnancy and newborn blood screening for congenital disorders), the Ministry does not produce patient information leaflets on prenatal screening. "Your Pregnancy/To Haputanga", a pamphlet produced by the Ministry of Health for distribution to pregnant women by maternity practitioners, does not make any reference to prenatal screening. A single reference to ultrasound scans (which are not described as a method of screening) emphasises that scans are a "specialist service", and not provided within the scope of standard maternity care. It is noted that "If you choose to have a scan, and there is no medical reason for it, you will be charged for the service" (2002:28). Given that NT scanning is in fact government-funded for each pregnant woman, it seems curious that ultrasound is presented in this way within this material. $^{158}$

In addition, the emphasis on individual responsibility for decision-making and healthy choices within this material (for example, several pages of the pamphlet outline the risks associated with smoking, alcohol, recreational drugs and the consumption of various foods during pregnancy), makes the omission of information on prenatal screening seem additionally strange, raising the question of why health consumers reading the pamphlet are not at least advised that they can expect their caregivers to provide information about prenatal screening. Again, responsibility for screening in terms of the provision of information and guidance for patients appears to have been

\footnotetext{
${ }^{158}$ This representation of ultrasound as only selectively available is at odds with patient literature produced by clinical bodies such as RANZCOG, where ultrasound is clearly conceived of and presented as a routine aspect of prenatal care.
} 
devolved by the Ministry to individual practitioners and their representative bodies, and as might be expected, each organisation similarly takes a different approach to the provision of informational material to pregnant women.

As noted earlier, NZCOM does not issue specific practice guidelines or produce informational material on prenatal screening. Campbell (2008) notes that independently produced written material is often given by midwives to women in their care. ${ }^{159}$ The College of General Practitioners (RNZCGP) similarly does not produce its own educational material for patients. No figures are available indicating the distribution of any particular information leaflets by midwives or GPs.

RANZCOG produces a leaflet entitled "Prenatal Screening Tests for Down Syndrome and other Fetal Conditions" (2006) which is made available to pregnant women under specialist care in New Zealand and Australia. ${ }^{160}$ There is not scope here to evaluate the content of the various leaflets able to be sourced in the course of this research, other than to note a not insignificant degree of variation in content and scope of this material, as might be expected within literature published by multiple sources. To date there does not appear to have been an attempt by practitioner groups to collaboratively standardise informational material for pregnant women, a fact which again may reflect the historic tensions between maternity practitioner groups in New Zealand.

\footnotetext{
${ }^{159}$ Campbell (2008) cites a pamphlet entitled "Ultrasound Scans in Pregnancy" produced by Women's Health Action Trust (WHAT) and the Maternity Services Consumer Council's "Choices for Childbirth" as examples of literature frequently given to pregnant women by midwives. She notes also that midwives often give women a copy of the Ministry of Health's leaflet "Your Pregnancy/To Haputanga".

${ }^{160}$ As RANZCOG was not willing to provide a copy of this patient information leaflet for the purposes of this research, this was accessed as a document available in the public domain through other sources.
} 
As discussed in chapter five, within clinical ethics the provision of written information to patients is typically espoused as the 'gold standard' of processes of informed consent. Participants' accounts within this study signalled a lack of satisfaction with the availability of written material on screening methods and options, and a concern that decisions were often made without sufficient information being provided. The lack of standardised patient information on prenatal screening in New Zealand can be seen to reflect the Ministry's historic reluctance to take a clear position on prenatal screening for Down syndrome, a phenomenon similarly reflected in the reliance on practitioners' various clinical bodies to direct the provision of screening 'in the field'. The reasons for this apparent ambivalence will be considered now.

\section{A mood for change: prenatal screening within recent public health discourse in}

\section{New Zealand}

This section turns to the representational politics of public health discourse on prenatal screening, considering the ways prenatal screening is differently constituted as a project of health within different domains. Drawing on literature within the New Zealand context, the discussion considers the existence of multiple narratives - private and public - about the aims of prenatal screening within public health discourse, and considers the extent to which representational ambiguity may be operationalised to circumvent core tensions between medical and social evaluations of the purpose of screening. The literature referred to here includes a number of recent clinical articles, informational material produced for the public by the National Screening Unit, ${ }^{161}$ and

\footnotetext{
${ }^{161}$ Please note that other than the 2007 report 'Antenatal Down Syndrome Screening in New Zealand', NSU does not appear to produce hard copy versions of the material posted on their website; therefore, the documents referred to here are necessarily sourced from the internet.
} 
a public information booklet produced as part of a recent public consultation project on "pre-birth testing".

In her analysis of prenatal ultrasound, Weir (1998a, 1998b) suggests that biomedical texts are characterised by a mobility of discourse, and she draws attention to the use of different 'registers' of discourse within medical and lay accounts. Such perspectives offer a useful starting point for exploring the protean character of recent discourse in New Zealand. Weir's theorising is positioned within recent social scientific work on the production and uses of medical knowledge, within which science is viewed as a form of culture, rather than a necessarily rational, culturally 'neutral' truth regime (for example, Franklin 1995, Lupton 2003 and Mol 2002). A key element of this perspective is the view that bio-scientific discourse is fluid and 'multiple', and wont to adopt (and exclude) discourses from other cultural domains. As Weir suggests, a number of different registers of discourse may exist coterminously, with variable prominence, within medicine as a 'restless, self-correcting truth regime' (1998b: 252).

With respect to prenatal ultrasound specifically, the flexible constitution of the unborn as, variously 'fetus' (i.e. as a biomedical concept) and 'baby' (which she identifies as a notion drawn from an 'extrascientific' domain) is a particular focus of Weir's work. Similarly, Taylor (1998) has highlighted sonographers' variable use of medical and extra-scientific registers in representing both the subject (i.e. fetus/baby) and, indirectly, the purpose of the scan (diagnostic vs. reassuring/kin-oriented) during the ultrasound scan. ${ }^{162}$ Within this and related work a distinction is drawn between medical and 'social' narratives of the unborn, with attention drawn to the cooption of

\footnotetext{
${ }^{162}$ Sonographers' practice is discussed further in chapter seven.
} 
social narratives for strategic purposes, for example by anti-abortion groups wishing to establish notions of fetal 'personhood'. In a similar way, this discussion suggests that what might be considered to be the social narratives attached to prenatal screening discourse (for example, narratives of individual choice and 'safety') may be strategically foregrounded within public health discourse, while medical narratives are given lesser prominence.

Weir has noted that flexibility of medical and social registers of discourse in the constitution and representation of the unborn is also evident in health promotion material intended for the general public, where, as she suggests, medical and popular understandings tend to merge: "The 'baby' in health care government is thus a hybrid of biomedical and popular knowledge" (1998b:254). However, little work appears to have been done specifically addressing public health discourse on prenatal screening as a matter of representational politics, and specifically, theorising the ambiguous framing of prenatal screening within this domain. A case study in chapter five explored the significance of representational ambiguity for one woman's experience of prenatal ultrasound; this discussion broadens this line of argument, suggesting that the manner in which prenatal screening is represented for the purposes of public information casts doubt on claims that the expansion of prenatal screening as a public health activity is driven primarily by imperatives of informed decision-making for pregnant women.

The constitution of prenatal screening as a public health concern brings to a head a key tension between medical and social understandings of health, and the therapeutic scope of medicine. As chapter five argued, as 'public health' projects, practices such 
as targeted screening and selective pregnancy termination do not obviously fit within categories of either 'prevention' or 'therapy', medical imperatives readily associated with other forms of population-based screening. In no body of literature are such tensions more clearly drawn than within the critique of prenatal screening made by disability activists, ${ }^{163}$ where the ideological gap between social and medical models is held up as an ongoing obstacle to the fulfilment of legislative commitments to inclusivity. Within public health discourse on prenatal screening the views of disability theorists have readily been marginalised, framed as the perspective solely of 'interest groups'. However, it may be that this domain is yet to fully recognise the extent of public valuing of disability. The accounts of participants in the interviews undertaken for this thesis suggest the possibility that a much broader public demographic may be ideologically aligned with a social model perspective of conditions such as Down syndrome. Certainly these accounts suggest that a distinction can and should be made between women's individual endorsement of a 'pro-choice' position on termination of pregnancy, and their broader moral comfort with the notion of targeted, population-based screening for Down syndrome as a statefunded project of health.

In theorising the strategies through which public acceptance of health initiatives is garnered within the public health domain, Lupton $(1993,2003)$ notes a lack of ideological transparency as a hallmark of public health discourse. In this analysis public acceptance is at least somewhat reliant on rhetorical management, and the reframing of notions of compliance with health management activities in terms of discourses of individual rights and choices: as she notes, "The rhetoric of public

\footnotetext{
${ }^{163}$ See particularly Shakespeare $(1998,1999)$ and also Parens \& Asch (1999).
} 
health discourse is such that the individual is unaware that the discourse is disciplinary" (2003:35). While a small amount of research is available which examines pregnant women's views of the ideological content of health information material, ${ }^{164}$ the representational politics of prenatal screening within public health discourse has not drawn much critical attention beyond the scope of the disability critique. This may be in part because the constitution of prenatal screening as a public health concern (i.e., the move to implement population-based as opposed to targeted 'high risk' screening) is a relatively recent phenomenon (Williams et al, 2005). It may also relate to the confounding ambiguity of prenatal screening discourse within this domain. This discussion will now consider examples of this discursive flexibility within a variety of texts recently produced within the public health domain in New Zealand. The literature discussed here comprises clinical articles, public information material produced by the National Screening Unit, and an information booklet produced as part of a recent public consultation project on "pre-birth testing".

As noted earlier, the emergence of prenatal screening as a public health concern is a very recent phenomenon in New Zealand, and there is a limited amount of published local material. However, within the literature available, it is interesting to consider the distinction between what might usefully be considered an epidemiological narrative of prenatal screening (i.e. that which is produced by and largely contained within a clinically oriented domain) and a public narrative, as constituted within material produced for the purposes of public information.

\footnotetext{
${ }^{164}$ See for example Rudolfsdottir (2000).
} 
In 2005, a news story entitled "Rise in Down syndrome babies blamed on lack of screening" (NZ Herald, 2005) ${ }^{165}$ reported concern within the public health community over the effectiveness of current screening for Down syndrome within New Zealand. Within the article Professor Peter Stone, a prominent NZ academic in the field of obstetrics and gynaecology, was cited as blaming increasing rates of Down syndrome in New Zealand on the lack of a co-ordinated national screening programme. The article reported an increase in babies born annually with the condition from 50 in 1996 to 80 in 2002. Prior to publication of this story, the issue of prenatal screening had drawn virtually no media attention within New Zealand, and literature evaluating Down syndrome screening practices in New Zealand was contained almost exclusively within the clinical/epidemiological domain.

While within the news story the incidence of Down syndrome in New Zealand is clearly framed in terms of an epidemiological view, whereby increasing incidence is 'blamed' by health officials on clinical shortcomings, it is interesting to note that this particular representation of Down syndrome is not a feature of informational material subsequently produced for the public during the review of prenatal screening practices. Within such material, screening for Down syndrome is typically framed according to narratives of 'safety' and choice, rather than in terms of incidence. ${ }^{166}$ Thus epidemiological concerns can be seen to be backgrounded within public information material. For example, in a 2007 media release by NSU, the aims of Down syndrome screening in New Zealand are described primarily in terms of the benefits for individual women rather than in relation to epidemiological goals: "The

\footnotetext{
${ }^{165}$ See appendix 10.

${ }^{166}$ In contrast, within clinical literature it is accepted that few therapeutic interventions are currently available for fetuses in utero, and the only 'choice' facilitated for women by the detection of abnormality is whether or not to terminate the pregnancy.
} 
key driver is to improve the safety and quality of screening practice for women in New Zealand" (NSU, 2007b, unpaginated). ${ }^{167}$

By contrast, within the clinical literature available which addresses the question of Down syndrome screening in New Zealand, the epidemiological aims of a Down syndrome screening programme appear to be indisputably tied to reducing the incidence of the condition within the general population. This is succinctly illustrated in Stone and Austen's 2006 report, where it is noted that the existing approach to screening in New Zealand has "failed to reduce the number of babies born with undiagnosed Trisomy 21" (2006:5). Within international epidemiological literature, the efficacy of screening programmes is measured in the first instance in terms of detection rates. ${ }^{168}$ This is similarly reflected within the New Zealand literature. For example, Stone (2005) discusses the efficacy of various approaches to screening, drawing attention to the recently implemented national Down syndrome screening programme in the UK as a possible model for "introducing nationwide screening with defined targets both for programme outcomes in terms of detection rates and national coverage" (2005, unpaginated). Considerations of incidence are also linked to financial imperatives. For example Chang (2006) states "For consideration of a national policy, cost-effective screening programmes will be significantly compromised if the participation rate is low. For a national policy for Down syndrome to be effective in New Zealand, the costs of the screening programme to detect a certain number of cases has to be balanced against the cost of managing missed or undetected cases in the population" (2006:94).

\footnotetext{
${ }^{167}$ See appendix 11.

${ }^{168}$ See for example Smith-Bindman et al (2003).
} 
Notions such as 'defined targets' and 'detection rates' would appear to suggest that such screening is driven, to a considerable extent, by an overarching imperative to reduce or at least cap the overall incidence of Down syndrome in the general population. However, such imperatives are certainly not made explicit within public information material produced for a lay audience. For example, within a 'questionand-answer' style media release by the NSU on the proposal to introduce a national screening programme, the question is asked "Does the Ministry aim to reduce the number of babies born with Down syndrome every year?", to which the reply is "No, this is not about reducing the numbers of babies born with Down syndrome. This is about ensuring that screening services offered to women during pregnancy do not cause unnecessary harm to women and their pregnancies" (NSU, 2006, unpaginated). ${ }^{169}$ The New Zealand Disabled Persons' Assembly (NZDPA) drew attention to this representational inconsistency in 2006 media release, ${ }^{170}$ raising concerns that in focussing on issues of 'safety' relating to the loss of normal fetuses, the review process failed to acknowledge the impact of targeted screening for Down syndrome on the disabled community (Scoop, 2006, unpaginated).

It is interesting to consider how the case for a national screening programme appears to have been differently constituted within each of these two domains. Additional evidence for the flexibility of public health discourse on screening may be seen in the variable representation of a national screening programme as, on the one hand, a necessary and inevitable response to problems with current practice, or on the other, a possible model, the implementation of which is to be directed by the public will.

Within literature in the clinical domain, the endorsement of a national programme is

\footnotetext{
${ }^{169}$ See appendix 12.

${ }^{170}$ See appendix 13.
} 
both clear and consistent. Proponents of a national screening programme for New Zealand have argued that in failing to implement a co-ordinated and formalised screening programme, New Zealand is falling behind the standard of prenatal care offered in other countries. ${ }^{171}$ Within such literature, the improvement of screening in New Zealand is clearly envisaged as necessarily occurring within the context of a formalised national screening programme. For example, Chang (in 2006) notes "The need for a unified national screening policy in New Zealand has never been greater and steps taken to identify the optimal screening policy are to be encouraged and hastened" (2006:92). Similarly, Stone (2005) states "Of greatest importance is the decision to commence a programme ... Few countries have properly constructed screening programmes as opposed to screening testing. There should be few impediments to proceeding with this now" (2005, unpaginated). Conversely, the NSU appears to take a more conservative tone within its public informational material, emphasising that any decision to implement a national programme would be dependent on "a great deal of detailed work" (2007b, unpaginated). Nonetheless, the unit's general endorsement of a national screening programme within this media release seems clear, evident in statements such as the following: "Implementing a national screening programme would help to ensure that women who choose to have screening are offered the safest and most reliable form of screening possible" (NSU, $2007 \mathrm{~b}$, unpaginated); here the logic of such a programme is essentially represented as self-evident. Furthermore, it is of note that the implementation of a national screening programme was the majority preference of the NSU's Advisory Group to the Ministry of Health, the committee noting that "the majority of members support the introduction of a nationally organised screening programme as the best way of

${ }^{171}$ See Stone and Austen (2006) for an inter-country comparison. 
ensuring consistently safe and effective screening that meets agreed standards, and is monitored and evaluated" (NSU, 2007a:54).

The informational material produced for the purposes of a recent public consultation project on pre-implantation genetic diagnosis (PGD) offers a third interesting example of the flexible constitution of prenatal screening within contemporary New Zealand public health discourse.

The history of public consultation on genetic technologies (including techniques of reproductive screening) in New Zealand offers much fodder for social scientific analysis and is a topic which warrants detailed examination in its own right, enquiry which is outside the scope of this thesis. ${ }^{172}$ However, the recent consultation undertaken by Toi Te Taiao/Bioethics Council of New Zealand, entitled "Who Gets Born? Pre-Birth Testing" (2007) usefully highlights the contradictory and ambiguous representation of screening noted earlier. For a start, the project's title is somewhat unclear, as the consultation relates primarily to the uses of PGD, a screening method occurring prior to conception and only within the context of IVF treatment, and not screening occurring during pregnancy. This conflation of entirely different applications of screening is confusing, due to the implication that pregnancy screening in general is being consulted on.

\footnotetext{
${ }^{172}$ In particular, a sociological analysis of the trajectory of public consultation relating to preimplantation genetic diagnosis (PGD) in New Zealand would be particularly fascinating and is yet to be undertaken. It is interesting to note that in response to questions relating to public consultation around prenatal screening in New Zealand, none of the participants for this study signaled that they were aware of the process of public submissions on PGD which had been carried out by the Ministry of Health in November 2004 (the year immediately prior to interviews being conducted), an observation which perhaps raises questions about the efficacy of public health consultation processes within New Zealand.
} 
Furthermore, the publicly available information booklet (or "Choicebook")

simultaneously represents screening practices as both 'routine' and dependent on public endorsement. For example, "Pre-birth testing, together with antenatal care, have become a routine part of ensuring the health of the mother and the baby" (2007:2). Similarly, it is notable that within a section entitled "Why test during pregnancy?", "standard practice" is listed as the first entry, a normative statement which again seems curiously at odds with the 'consultative' approach claimed for the project. In spite of similarly normative representation of pregnancy screening throughout the booklet, the material emphasises the significance of the public's role in determining the acceptability of such tests, and in directing their implementation: "Deliberating on an issue means people coming together as informed citizens and taking responsibility for making choices about how they want to live, how they want to act together, and how they want their government to function" (2007:2), and as an additional example: "This choicebook is intended to encourage rewarding discussions between people about the issues of pre-birth testing. When we know what others believe about the issue, we can have reasoned respectful dialogue about how best to respond to the challenges and opportunities posed by pre-birth testing" (2007:9). It is, then, curiously ironic that within the report published by the Bioethics Council at the conclusion of the project, a key finding of the consultation is dismissed; this relates to the use of PGD for the non-medical sex selection of embryos. ${ }^{173}$ In spite of the majority of participants in the consultation indicating that they would not endorse this practice, the council recommended that the Ministry of Health repeal an existing ban on social sex selection, noting that "As far as sex selection for the purposes of 'family balancing' is concerned, we have not heard sufficient cultural, ethical or spiritual

\footnotetext{
${ }^{173}$ A process frequently referred to as 'family balancing' within popular media reporting on PGD.
} 
concerns to justify banning it - providing PGD is undertaken at the parents' own cost. We think further investigation of the reasons for apparent public concern about the use of PGD for sex selection is warranted. The reasons for people's concerns about sex selection for family balancing remain unclear" (2008:51).

The Bioethics Council's apparent disregard for public opinion on this particular issue may be, I would suggest, emblematic of a broader reluctance by health officials to engage with and address public attitudes when they are predicted to be at variance with specific policy imperatives. Certainly the recent review of Down syndrome offers compelling evidence of an at best lukewarm commitment to public consultation in this area; this appears to be the case in spite of recognition within the public health community of a historic lack of public engagement on this issue. In an advisory document produced for the Director General of Health, the National Screening Advisory Committee notes that "to date, there has not yet been formal consultation with consumers of maternity services, the disability sector or the general population in relation to antenatal screening for Down syndrome" (NSAC, 2007, unpaginated). The subsequent final report on Down syndrome screening in New Zealand to the Ministry of Health (NSU, 2007a) underscores the desirability of consultation in determining the social acceptability of a formalised national screening programme, noting that: "To encourage wider societal debate, the Government should consider carrying out public consultation to determine the public's views on whether or not there should be an antenatal Down syndrome screening programme" (NSU, 2007a:4). However, several years subsequent to the review, and with changes to existing practice currently under implementation, it seems highly unlikely that public opinion will now actually be sought on this issue. As I will suggest, given the outcome of the review process, 
it seems reasonable to question the extent to which 'wider societal debate' on prenatal

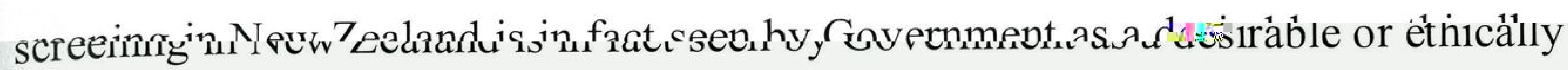
necessary element of policy development in this particular area.

\section{Future directions: a national Down syndrome screening programme by any other name?}

In spite of evidence of historic endorsement for the implementation of a national screening syndrome for Down syndrome in New Zealand by both the National Screening Unit, and prominent clinicians such as Professor Stone, this has not been the official outcome of the recent period of review of current practice.

In August 2008 the National Screening Unit announced the intended introduction of a range of changes to existing screening practices. Proposed changes include the implementation of new screening 'pathways', the provision of information (presumably nationally consistent) to pregnant women and their partners, the development of protocols and standards for clinicians and laboratories, and monitoring of these service providers. ${ }^{174}$ One immediate change from 2008 was the temporary implementation of an additional maternal blood test for Down syndrome (MSS2), pending future changes to the methods offered currently.

In addressing what might be considered technical and administrative problems with recent screening practice in New Zealand, including questions of accuracy and reliability, and rectifying inconsistencies in the provision of screening services, these changes will undoubtedly have real benefits for pregnant women in terms of reducing some of the uncertainties attached to the experience of screening. In particular, the fully-funded implementation in 2010 of more accurate screening methods (chiefly a 'combined' approach of NTS plus maternal blood tests) should go some way to

\footnotetext{
${ }^{174}$ See appendix 14
} 
mitigating the problem of 'false negative' and false positive' diagnoses, and the stress associated with this experience. Such changes should also have a positive benefit for pregnant women in terms of a reduction in the rates of miscarriage associated with unnecessary referral for invasive diagnostic testing (i.e. amniocentesis).

Furthermore, the intention to improve provision of information to women would likely be welcome news to many participants within this research, for whom dissatisfaction with this issue appeared to constitute a core 'theme' of their experience. As many of these changes are yet to be implemented, the content and rhetorical character of this informational material remains to be seen (in particular the extent to which screening is institutionally endorsed).The stipulation that 'Women who choose not to be screened will be supported in their decision' (NSU, 2008), ${ }^{175}$ does however represent a significant departure from previous material produced in this domain which, as previous chapters have argued, has historically presented acceptance of screening as an unproblematic personal and public good. If such statements signal a shift towards official sanction for the choice to refuse screening, it will be interesting to chart the ways this choice is presented within subsequent public health material.

However, this apparent 'eleventh hour' retraction of official endorsement for a national screening programme in New Zealand does appear to leave a number of unresolved issues. Given that consideration of the implementation of such a programme within New Zealand had been a specific brief of the review process, the

\footnotetext{
${ }^{175}$ Appendix 14, page one.
} 
lack of an official and public decision by the Ministry of health against this recommendation seems rather curious. What is the public to make of this outcome?

The framing of changes to screening practice in New Zealand in terms of "quality improvements" suggests an emphasis on the content rather than the overall form or approach to screening, when previously there had been considerable impetus for a major restructuring of the existing approach via the implementation of an official, nationally-co-ordinated screening programme for Down syndrome, such as that which now exists in the UK. It is not clear how the modification of existing practices now being implemented by the health Ministry differs materially, or in intention, from the measures proposed for consideration of an official Down syndrome screening programme. ${ }^{176}$ Arguably, an emphasis on co-ordination and national consistency would seem to suggest a will to formalise the practice of universal screening, a process which it appears will be directed by the National Screening Unit. ${ }^{177}$ In the absence of an official explanation for the eventual decision against a national screening programme it is only possible to speculate on the rationale informing this approach.

As argued earlier in the chapter, the constitution of prenatal screening as a public health concern brings together a number of contradictory understandings of health, disability and the purpose of medical care. I would suggest that the outcome of the review reflects, at least to some extent, an attempt to bypass the tension between 'medical' and 'social' evaluations of the purpose and value of prenatal screening

\footnotetext{
${ }^{176}$ For example by Stone \& Austen, 2006, and within the NSU report (2007).

${ }^{177}$ See appendix 14, page 2.
} 
which problematise the straightforward presentation of prenatal screening in public health discourse as a practice for promoting the general health of the population. In this analysis, public debate on the issue may be seen to present a potential obstacle to the implementation of what are considered to be epidemiologically sound and desirable goals. Certainly, the question of public perception appears to have been recognised during the course of the review as an issue requiring careful presentation within the context of potential changes to current practice, as the following comment suggests:

International literature suggests that a national screening programme will lead to a reduced incidence of Down syndrome, and other fetal anomalies. There is concern that the establishment of a national screening programme will imply that the aim is to reduce the incidence of Down syndrome.

(NSU, 2007a:55)

The advisory group's recommendation that "the Ministry of Health should explicitly state that the aim of antenatal screening is not to decrease the incidence of Down syndrome" (2007a:65) lends further weight to this suggestion. However perhaps the most compelling evidence for a claim that a national screening programme for Down syndrome in New Zealand may have been introduced 'though the back door' can be found in a 2009 media story on the proposed changes, within which Professor Stone is quoted thus:

There will in effect be national screening but due to complex sensitive political factors the advice from the national screening unit and the Director general of Health to the past and current Ministers of Health was not to call this a national screening programme but 'quality improvement'. ${ }^{178}$

Putting aside issues of representation and questions around the ethics of the rhetorical 'flexibility' of prenatal screening discourse within recent public health material, I would suggest that the changes to current screening practice in New Zealand would likely fall far short of addressing a number of other concerns expressed by

\footnotetext{
${ }^{178}$ See appendix 15.
} 
participants within this thesis, in particular those relating to the extent to which screening is currently experienced as an authentic 'choice' within the climate of contemporary pregnancy care. The co-ordination of practices within the existing model fails to address broader issues of choice and consent, including the normative impact of a culture of routine screening on women's decision-making experiences. As discussed in chapter two, a large body of literature - both clinical and social scientific - clearly indicates an inverse relationship between routinised screening and informed, deliberative decision-making in pregnant women.

While an emphasis on improving the 'safety' of current practices suggests a commendable intention to provide a higher standard of screening services in New Zealand, the investigation of screening as a 'lived experience' would have provided invaluable insights into what it is that pregnant women themselves seek and value in their engagement with prenatal screening. As the empirical content of this thesis suggests, for those on the receiving end of such practices, notions such as 'safety' and 'choice' may be conceptualised more broadly than in terms of technical reliability and consistency, but appear to relate significantly to the extent to which individual women feel able to make decisions in accordance with personally held values. At least as much as the wish for technical accuracy and the provision of information, this appeared to be regarded as a priority for ethical screening practice. The lack of public input into the future shape of prenatal screening for Down syndrome in New Zealand speaks of perplexing disregard for the insights produced by a now considerable body of qualitative research in this area, all of which highlights, in one way or another, the extent to which prenatal screening ought to be viewed as an ethically complex 'special case', in a way which is appropriately responsive to the situatedness of 
women's pregnancy experiences, as well as their demonstrated preferences as the primary 'consumers' of these technologies.

Irrespective of whether a nationally co-ordinated programme for Down syndrome screening is now, in effect under implementation in New Zealand, an opportunity appears to have been missed during the course of the review to investigate the possibilities for an acceptable middle ground between epidemiological and economic imperatives, and public attitudes and expectations around the value of screening for Down syndrome. As chapter eight will suggest, as the primary 'consumers' of prenatal screening technologies, pregnant women themselves appear to regard the assurance of mechanisms for public involvement in policy development in this area to be an important and necessary element of ethical practice. 


\title{
Chapter Seven
}

\section{Fetal imaging as 'spectacular medicine': exploring the boundaries of contemporary medical uses of prenatal ultrasound}

\begin{abstract}
Although there are many indications for performing obstetric ultrasonography as outlined by the National Institutes of Health Consensus Development Conference and adopted by the American College of Obstetrics and Gynaecology, we believe that being pregnant is reason enough. (Nyberg et al, 2003)

I'd be saying yeah, have a scan, it'll be really nice for you whether or not your baby's fine.

(Emily)
\end{abstract}

As suggested in chapters three and four, women's own accounts of prenatal screening (and specifically ultrasound scanning) suggest that fetal imaging is viewed by those on the 'receiving end' of this technology as having more than one use or function; it is experienced variously as both a social and a medical encounter. This flexible conception of scanning is well captured in Emily's comment above, in which it is clear that the diagnostic imperatives of the procedure are seen as quite distinct (and functionally divisible) from those aspects of the experience relating to social or, more precisely, 'extra-scientific' expectations.

A substantial body of research (both medical and social scientific) demonstrates that pregnant women frequently value scans at least as much for reasons "outside the realm of traditional medical decision-making" as for their diagnostic capability (Stephens et al, 2000:603). ${ }^{179}$ The ambiguous nature of women's engagement with prenatal ultrasound has been considered in a substantial body of social scientific

\footnotetext{
${ }^{179}$ See also Gudex et al (2006), Harris et al (2004) and Garcia et al (2002).
} 
work, and has been discussed elsewhere in this thesis. However, I would suggest that as a core tension underpinning the phenomenon of prenatal screening, this question of the relationship between the social and medical 'uses' of this technology is yet to be fully addressed with respect to clinicians ' uses of prenatal ultrasound. ${ }^{180}$ While it is clear that this technology is widely embraced within the obstetric institution, given the contested evidence for the value of routine prenatal scanning in prenatal care, ${ }^{181}$ it seems important to scrutinise the clinical practices and discourses which surround its use. In doing so, several questions emerge relating to the nature of fetal scanning as a medical project. What lies behind the apparent clinical enthrallment with fetal life as a "fascinating puzzle in and of itself" (Franklin, 1991:193)? To what extent might clinicians themselves play a role in cultivating and supporting popular misapprehensions around the purpose of diagnostic imaging?

This discussion will explore recent innovations in fetal imaging which arguably demonstrate a subtle shift towards non-diagnostic practices, and argue that the answer to such questions may lie in the 'extra-scientific' aspirations which clinicians themselves attach to the use of prenatal ultrasound.

\section{Prenatal ultrasound: channelling 'desire'?}

Drawing on her study of couple's experiences of fetal ultrasonography, Margarete Sandeloswki observes that this technology has a profound appeal both inside and outside 'the clinic', as something which "both physicians and childbearing couples do not want to do without and cannot resist" (1994:263). While this particular work can be seen to focus in more depth on pregnant women than on clinicians per se, her

\footnotetext{
${ }^{180}$ Prenatal ultrasound is primarily performed by obstetricians and sonographers, and for this purposes of this discussion 'clinicians' should hereafter be assumed to refer to these types of practitioners unless otherwise indicated.

${ }^{181}$ See chapter three.
} 
conception of prenatal ultrasound as a 'channel of desire' (1994:262) ${ }^{182}$ offers a useful starting point for examining the form and content of medicine's apparent love affair with this technology. In using the term 'desire' here I am simply setting up an overt distinction between clinically instrumental uses of such technologies (for example, the use of ultrasound exclusively for diagnostic purposes) and forms of engagement which reflect 'extra-medical' expectations (such as the desire to 'meet' or ascribe personality to an unborn baby, etc). While such distinctions do not reflect the complex imbrication of medical and cultural norms which this chapter suggests shape both lay and professional uses of prenatal ultrasound, framing the use of this technology in terms of various 'desires' or intentions, rather than merely for the 'objective' extraction of clinical data, makes it possible to explore clinicians' various responses to and uses of this technologies more freely.

An exploration of the role of desire or, more broadly, pleasure, in the use of medical technologies raises uncomfortable questions both for the institution of medicine and for advocates for women's right to direct their reproductive care. As Foucauldian theorists would argue, pleasure needs to be viewed as intractably political, and always with an eye for its relationship to broader dynamics of power, that is, the extent to which it may be structured relative to various cultural norms. For clinicians, the notion that their practice might be, to some extent, shaped by various culturally rather than medically determined desires, presents a profound challenge to ideals of professionalism and scientific objectivity. For feminist theorists, women's demand for and consumption of prenatal ultrasound raises difficult questions about agency and autonomy. Framing women's engagement with reproductive technologies in terms of

\footnotetext{
182 Sandelowski cites this expression to Ewen \& Ewen's (1982) discussion of the significance of massproduced images within American popular culture.
} 
disciplinary power and practices, Foucauldian theorist Jana Sawicki suggests that the implementation and embrace of such technologies involves the production of novel objects/subjects of medical and popular enquiry, a process which she argues operates by "inciting and channelling new desires" (1991:83, cited in Farquhar, 1996:5). The notion that women's uses of and desire for this technology might in fact be prescribed or 'channelled' in this way, shaped by institutional rather than individual intention, sits uneasily with the view of pregnant women as 'in charge' of their own pregnancy care. As Farquhar (1996) suggests, women's widespread embrace of prenatal scanning in spite of questions around its medical utility can be seen to persist as an 'uncomfortable truth' for those seeking to hold back unnecessary medical involvement in maternity care. In both cases, I would suggest that this discomfort around the specifics of extra-scientific discourses and 'uses' of fetal imaging has resulted in a notable reticence on this issue within clinical and feminist literature. ${ }^{183}$

Having positioned clinicians' uses of fetal imaging in terms of desire, the discussion will now consider how some recent developments in the technology and application of prenatal scanning.

\section{Recent developments in fetal imaging}

Clinical literature on prenatal ultrasound (both past and present) frequently appears to presume an equivalence between visual clarity and technical 'truthfulness', an

\footnotetext{
${ }^{183}$ For example, Petchesky argues that pregnant women "frequently express a sense of elation and direct participation in the imaging process" (1998:279), but does not specify how this sense of agency might be materially enacted (i.e. as a 'use' of this technology). Similarly, Farquhar signals but does not elaborate on women's "appropriation of sonogram technologies" (1996:171). It should be noted however that work such as that undertaken by Mitchell \& Georges $(1997,1998)$ and Taylor $(1998)$ does frame women's engagement with fetal images in instrumental terms, i.e. in terms of a 'use'; specifically, as a means to strengthen various familial and social bonds (for example to secure a male partner's interest in the pregnancy).
} 
observation which is borne out in examples such as the following from a contemporary obstetric textbook:

Ultrasonography provides a window of unsurpassed clarity into the gravid uterus. Over the past 10 years, high-resolution real-time ultrasound has undeniably become the most prevalent and accurate means of obstetrical imaging, capable of providing exquisite detail regarding the fetus and the intrauterine environment.

(Pretorius \& Mahony, 1990:1)

Such evaluations lucidly illustrate the extent to which positivist conventions around the valuing of visually based knowledge operate as a potent meta-discourse within obstetric knowledge. ${ }^{184}$

As I will suggest, the assumption that to see more is necessarily to know more appears to be as deeply embedded in contemporary medical discourse and practices of fetal imaging as when ultrasound was first hailed as offering unimpeded visual access to the formerly secret life of the fetus in the nineteen-sixties (Oakley, 1984). As will be discussed later, recognition that rather than verisimilitude ultrasound imaging only ever offers a limited 'viewing paradigm' (Maher, 2002:105) is curiously absent within clinical literature. This appears to be no less true of the most recently developed applications of prenatal imaging: the use of three and four dimensional scanning.

The basic modality of diagnostic prenatal ultrasound has not changed significantly for the last two decades. Two-dimensional (2D), real-time ultrasound produces the characteristic 'black and white TV' images in motion familiar to most women who have undergone pregnancy scanning. This technique permits the measurement of fetal

${ }^{184}$ A point argued at length in feminist critiques of medicine's 'visual culture'. (For examples, see Oakley 1984 \& 1986, Duden, 1993, and Petchesky, 1987). 
size and shape in two planes, as well as the observation of fetal movement simultaneously as it occurs in utero. However, while 2D scanning continues to be widely utilised by sonographers and obstetricians as the primary method of detecting fetal anomalies (Nyberg et al, 2003:xiii) and regarded, as Campbell suggests (2002:2) as "the cornerstone of antenatal imaging for the foreseeable future", in recent years considerable research resources have been directed towards the development of newer, and more specifically, 'true-to-life' imaging techniques. Three and fourdimensional (hereafter 3D and 4D) scanning modalities are now in comparatively wide use within the clinical setting, as well as within the commercial sector, where non-diagnostic scanning services have flourished due to popular demand for specific images and videos of their fetuses, a phenomenon increasingly referred to as 'keepsake' imaging. ${ }^{185}$ The (comparatively) recent development of $3 \mathrm{D}$ and 4D scanning modalities (see below), and specifically the case made for their widespread use (particularly within the clinical setting), provides compelling evidence for the argument that clinicians' enthusiasm for fetal imaging may be as much to do with the 'imaginative appeal' (Sanger, 2008) of such techniques as any pragmatic medical value.

Fishman et al describe 3D ultrasound imaging as a technique which "reformats conventional imaging data into a series of images that closely resemble the original structure", a process which is "entirely dependent on computers" (1991:321). While this method has been in use for around a decade and is now used by a 'vast' number

\footnotetext{
${ }^{185}$ See for example Wax \& Pinette (2006). Demand for this service appears to be most evident within the USA, where business with names such as "Womb with a view" and "Peek-a-boo baby" have opened in pubic spaces such as shopping centres (Rados, 2004). This type of 'user-pays' nondiagnostic scanning is also available in the United Kingdom, the market niche there generally being filled by private medical clinics which make video footage of scans available for purchase (Chudleigh, 1999).
} 
of clinicians (Tarsa et al, 2006), there is recognition within ultrasonographic literature that the use of 3D imaging in day-to-day practice is yet to be extensively evaluated.

Nonetheless, the case is frequently made that 3D scanning offers a more 'state-of-theart' approach to diagnostic imaging, and there certainly appears to be widespread clinical enthusiasm for its use in prenatal scanning. ${ }^{186}$ As a more recently developed technique, 4D ultrasound is essentially 3D dimensional imaging with the addition of real-time motion (i.e. with time/motion constituting the fourth 'dimension'(Campbell, 2002).

Both in tone and content, clinical discussion of these techniques reveal a somewhat startling degree of enthusiasm for the images produced, and for the impact these are seen to have on prospective parents during the scanning process. For example, as a comparatively recent example of clinical commentary on the use of 4D imaging, Campbell's discussion (from an editorial article entitled "4D, or not 4D: that is the question") offers a fascinating resource for exploring clinical understandings of the purpose of fetal imaging. The following account of parents' reaction to this form of scanning is of particular note:

I am sure I reflect the views of all practitioners using 4D equipment that both maternal and paternal reaction to the moving $3 \mathrm{D}$ image is something we have not previously encountered. I have seen fathers kiss the screen or (more appropriately) their partner's abdomen in an ecstasy of recognition and pleasure. The term 'scanning for entertainment' is surely an insulting one with which to describe the natural desire of parents to see and know and love their baby before birth. (Campbell, 2002:2)

These remarks can be seen to typify the 'extra-medical' dimension of much clinical discourse around prenatal scanning. This brings to mind discussion elsewhere in the

${ }^{186}$ For examples of this see especially Pretorius et al (2003). 
thesis about clinicians' tendency to represent the fetus as an already-born baby (Sandelowski,1994a) and to encourage prospective parents to do the same;

furthermore as I will argue later, this enthusiasm can be seen to reflect a somewhat fetishised conception of fetal life. As Weir observes, "there is a general incitement to read foetal sonograms as baby pictures; the research literature and clinical practice facilitate that incitement rather than standing outside it" (1998a:84). This certainly seems to be true of contemporary clinical discourse around the use of 3 and 4D imaging.

The addition of the 'real-time' aspect of 4D ultrasound appears to have staked out a new area of medical enquiry - the investigation of fetal behaviour. I would suggest that practices around this field of interest signal a shift within prenatal imaging away from diagnostic imperatives towards a more 'social' conception of the purpose of fetal imaging. As one prominent obstetric sonographer notes, "to me the biggest gains in terms of 4D scanning are in two relatively unexplored areas: parental behaviour and fetal behaviour" (Campbell, 2002:2). With respect to the latter specifically, Campbell cites the following examples as evidence of the clinical applications of this technique:

In addition to yawning, sucking and swallowing, which have previously been described by 2D imaging, it is now feasible to study a full range of facial expressions including smiling, crying, and eyelid movement.

(Campbell, 2002:3)

Within this and similar literature on 4D scanning, the clinical utility of such observations is often not clarified, and, I would suggest, appears to turn on the presumed benefits of variously humanising fetuses in ways seen to enhance parents' enjoyment of, and specifically their sense of connection with, their fetuses. This notion of prenatal parental bonding (often referred to as 'maternal-fetal bonding' 
within the clinical literature) can be seen to function as a key explanatory discourse attached to the use of 3 and 4D imaging (see later). While the attribution of character and personality to scanned fetuses is a practice which predates the emergence of the 4D technique, ${ }^{187}$ it seems clear that the addition of three-dimensionality is seen not only as a clinical benefit, but as a means of enhancing this extra-scientific aspect of scanning as a clinical project.

It is notable that literature on 3D and 4D imaging frequently emphasises women's positive view of these techniques, sometimes appearing to claim this enthusiasm as 'evidence' that bonding has successfully been elicited. For example, a recent comparative study of pregnant women's responses to 2D versus 3D scanning concludes that:

3DUS has a more positive impact and makes it possible for mothers to better visualize their developing fetus and baby than 2DUS. Mothers showed images of their baby to friends and relatives more frequently with 3DUS and used more enthusiastic descriptions of what they saw. 3DUS, by making it possible to better visualize the baby, may have the potential benefit of increasing the bonding of mothers to their expected newborns and strengthening the support system for their families. (Ji et al, 2005)

Clinical commentary of this nature raises the question of the extent to which popular enthusiasm for evolving imaging technologies may be co-opted within clinical discourse as a medical justification for its use. Furthermore, the assumption of a correlation between pregnant women's sharing of more realistic fetal images with family and friends and enhanced social support would appear to suggest an

\footnotetext{
${ }^{187}$ See Mitchell \& Georges (1998) for a discussion of this practice within the context of conventional (i.e. 2D) scanning, in particular clinicians' whimsical commentary on fetal behaviours such as 'dancing' and 'partying' during scans.
} 
assumption that there is no limit to the potential benefits of ultrasonically mediated bonding.

I will return to the question of 'fetal-maternal bonding' shortly, however firstly it may be useful to examine a secondary appeal to the value of 3D and 4D imaging techniques within clinical literature, the notion that these techniques are more diagnostically accurate than older modalities.

Each of the extracts from the clinical literature discussed above underscores, in slightly different ways, a presumption of equivalence between novelty and superiority in imaging technology. While it might make intuitive sense to assume that $3 \mathrm{D}$ images would necessarily be more 'realistic' and recognisable than the 'flat' appearance of an image in two dimensions, according to the clinical literature, the general diagnostic superiority of 3D imaging is by no means clear. Although 3D imaging is recognised as particularly useful for analysing specific defects following detection, ${ }^{188}$ as Scharf et al note, "the image information acquired by $3 \mathrm{D}$ ultrasound is nearly always inferior to the image information obtained by conventional 2D imaging"(2001:333). Sedgmen et al (2006:245) similarly refer to the overall diagnostic inferiority of 3D versus conventional scanning, noting that the evidence that this method enhances maternal attachment is "equivocal".

As Scharf et al observe, the difference between 2D and 3D imaging is less a question of accuracy than the brain's ability to 'fill the gaps':

\footnotetext{
${ }^{188}$ For example, Merz (1997) notes that 3D imaging allows more in-depth analysis of specific conditions such as spina bifida
} 
Both methods work with identical data sets. It is merely the human brain that perceives the given extent of the prevailing disorder in a faster and more complete way if it is presented in the form of $3 \mathrm{D}$ images. Therefore, the literature that recognises a visual benefit derived from 3D depictions of a malformation is describing a profoundly different quality than it pretends to comprehend; it is not the quality of the physical visualization of a structure which is rated but the psychological effect which is generated in the human brain by the image presentation. (Scharf et al, 2001:339)

As the authors go on to suggest, this fit between the way we are accustomed to interpreting visual images and the 'plastic image presentation' produced by the 3D technique may go a considerable way to explaining the general fascination with 3D imaging (2001:340).

However, such insights are uncharacteristic of the bulk of clinical literature on prenatal ultrasound, within which the superiority of three dimensions over two as a 'state-of-the-art' modality appears to be taken as read. This assumption of the selfevident nature of clincial data as 'truth' is arguably seen more broadly in statements such as that by prominent sonographic clinicians such as Nyberg et al (2003) which head the chapter. Such commentary on the appropriate use of prenatal scanning would seem to signal a profound contradiction within medical discourses around the nature (and value) of clinical evidence. To read a fetal image as a 'natural fact' ignores the reality that any 'meaning' this image may hold is entirely imported and does not arise from the data itself. As Taylor notes:

Nothing about the device itself dictates...that women undergoing ultrasound examinations should want and be encouraged to bring along husbands, boyfriends, or other family members or friends; that they should be shown the fetus on the screen; that seeing it should be understood as a means of effecting maternal "bonding"; that the sonographer should provide a narrative of the 
baby's anatomy and offer to determine its sex, or give the pregnant woman a videotape or "snapshot" image to take home. (Taylor, 2002:369)

Nonetheless, clinical literature is frequently marked by an apparently contradictory conception of fetal images as at once neutral 'data' and materially 'present' entities with a deeply affecting appeal. I would suggest that within the specific visuality expressed in medical readings of fetal images, the maintenance of the status of the fetus as an extra-scientific, fetishised object has become increasingly necessary to the 'logic' of scanning as a medical event. This conception of the fetus as a clinical 'fetish' is in itself nothing new within social scientific literature, having first been proposed by Petchesky in her influential discussion of prenatal ultrasound (1987).

Viewing the imaged fetus in this way offers a useful way to account for the apparent cognitive dissonance between social and medical discourses around the purpose and scope of fetal scanning. As she suggests, in fixating on a decontextualised image, imperatives of 'fact' and deductive logic are readily overshadowed by intentions imported from outside the sphere of strictly defined clinical goals:

"Evidence" shades into fantasy when the fetus is visualised, albeit through electronic media, as though removed from the pregnant woman's body, as though suspended in space. This is a form of fetishization, and it occurs repeatedly in clinical settings whenever ultrasound images construct the fetus through "indications" that sever its functions and parts from their organic connection to the pregnant woman...Indeed, the very idea of "bonding" based on a photographic image implies a fetish: the investment of erotic feelings in a fantasy. (Petchesky, 1987:277)

Petchesky's remarks can be seen to have foreshadowed the trajectory between a fetishised view of the fetus and the emergence of discourses of fetal bonding which I would argue have come to so prominently shape contemporary practice. These will be discussed now. 


\section{Merging fact and fetish: the evolution of fetal-maternal 'bonding' discourse}

As suggested earlier, clinical literature often presents 'maternal-fetal bonding' as a key benefit of prenatal ultrasound. ${ }^{189}$ Within recent literature (and in particular, that which concerns 3D and 4D techniques) this notion of bonding appears to be increasingly conceptualised as a therapeutic aspect of fetal imaging, and specifically as a psychological benefit for pregnant women and their families. ${ }^{190}$ The fostering of such bonds is also seen as a vehicle for the protection of fetal health, through discouraging pregnant women from harmful or 'fetal-averse' behaviours such as smoking. ${ }^{191}$

The notion of fetal-maternal bonding is not new within the context of prenatal scanning, however I would suggest that within clinical literature, discourse around the presumed clinical benefits has recently begun to gain momentum, and that this has occurred in line with the development of new imaging techniques (and the evolution of the broader field of 'fetal-maternal medicine'). In order to trace the origins of this emerging field of medical interest it is useful to consider the inception of the notion of maternal-infant (i.e. newborn) bonding as a medical concern in the 1970s. At this time, a number of clinicians in the field of pediatrics and obstetrics began to hypothesise that attachment-oriented behaviour at or after birth (such as immediate close physical contact between mother and baby) might have a long-term positive effect on parent-child relationships. ${ }^{192}$ The promotion of such bonds has subsequently become a widely accepted goal of contemporary neonatal care (although Tessier et al (1998) argue that few studies substantiate the bonding hypothesis). The notion that

\footnotetext{
${ }^{189}$ See for example Villeneuve et al (1998), Baillie et al (1997), Ji et al (2005), and Rustico et al (2005).

${ }^{190}$ See for example Pretorius et al (2003) and Campbell (2002).

${ }^{191}$ See for example Ruiz (1992).

${ }^{192}$ See for example Klaus et al (1972) and Kennel et al (1974).
} 
such bonds might be initiated prior to birth, via ultrasound imaging, was first

proposed in the early 1980s in an article which continues to be cited in contemporary

clinical research on the use of ultrasound. As the earliest clinical treatise on fetal-

maternal bonding, Fletcher \& Evans' discussion can be seen to exemplify the ardent

enthusiasm for prenatal imaging which I have earlier discussed:

Perhaps a new stage of human existence, "prenatality", previously only mirrored in poets' and mothers' dreams about the fetus, will be as real to our descendants as childhood is to us. Liley, the father of fetal therapy and a pioneer in ultrasonography, wondered as long ago as 1972 if the human fetus had a personality. For these and other reasons, physicians and their colleagues in obstetric and reproductive fetal medicine should not be surprised to find themselves attracting the most careful human scrutiny and imagination. Of such stuff are many human dreams made.

(Fletcher \& Evans, 1983:393)

This example of the intermingling of scientific frontierism with a kind of

metaphysical sentimentality foreshadows the romanticised notions of fetal life which I would suggest continue to be found in contemporary clinical literature.

Within such material the claim is often made that the demand for medical assistance with bonding originates from parents themselves, the discourse appearing to position parental approval as itself a clinical rationale for the practice. For example, Pershutte (1995) notes that "the influence of sonography upon parent emotional wellbeing is strong. This is evidenced by the passion with which patients embrace the still frame images that we provide them" (Cited in Baillie et al, 1997:1223). Such claims appear to rest on the presumption of an inherent benefit for pregnant women in forming an emotional connection to their fetuses. ${ }^{193}$ However, while clinical literature may often

\footnotetext{
${ }^{193}$ Further to this it is interesting to note that 'psychological support' is listed among the clinical objectives of routine ultrasound scanning by the Royal College of Obstetricians and Gynaecologists in the UK (see Baillie et al, 1997)
} 
frame the formation of affective 'bonds' as a kind of natural extension of

obstetricians' role, the extent to which such bonding exists as a psychological 'need' among pregnant women themselves is far from clear. Indeed, as Garcia et al (2002) note, in surveying current ultrasonographic texts little evidence of clinically meaningful benefits is able to be found. This paucity of data to support practices which enhance bonding is in fact sometimes noted within this area of research (albeit as a minority perspective). For example, Muller (1996) notes that no studies exist which demonstrate a sustained increase in feelings of attachment following prenatal ultrasound. As she observes, "the primary assumption underlying the promotion of prenatal attachment has yet to be supported by empirical evidence" (1996:161). With respect to 3D/4D scanning specifically, several recent studies cast doubt on claims of increased bonding, and note that irrespective of women's positive responses to these techniques, no significant differences in maternal attachment are measurable between older and newer methods of scanning. ${ }^{194}$ Indeed, as Sedgmen et al note, "Current findings suggest that the quality and clarity of the image may not be critical for maternal-fetal attachment" (2006:250). Given that improvements in the 'realism' of the images produced by the newer techniques is so often advanced as the basis of enhanced bonding, it is difficult to imagine what other case, precisely, might be made for continued developments in this field; as the authors intriguingly note; "It has... been suggested that the shadowy forms of a 2D image may intrigue the mother more and allow for personal elaboration, while the more graphic 3D image leaves little to the imagination" (2006:250).

\footnotetext{
${ }^{194}$ See Rustico et al (2005) and Sedgmen et al (2006).
} 
While the perversity of aspects of medical bonding discourse can be taken as a sign of the view of fetal imaging as one of the more 'lighthearted' tasks sonographers may get to perform, such discourses do have material ramifications for pregnant women. It therefore seems necessary to scrutinise the ethics of the coupling of bonding discourses with the practice of prenatal screening, and to reflect on the extent to which it is appropriate to configure an arguably pseudo-scientific 'fetish' in terms of clinical benefit. Irrespective of the actual clinical utility of 3D and 4D techniques, it seems important to recognise that however much 'bonding' may be conceived as a legitimate medical goal, the notion that pregnant women should form an emotional connection with their fetuses prior to birth is a highly contestable one. As discussed earlier, sonographers appear to prize their role in facilitating the 'meeting' of prospective parents and fetuses. However, is it ethical to actively mediate a sense of emotional connection in this way? The costs of encouraging a conception of fetal personality and the various 'claiming behaviours' which parents are encouraged to show (Sandelowski, 1994a:272) are not well understood. However it is clear that the notion of bonding valorises a form of unconditional maternity which is at odds with the provisionality underpinning scanning as a method of prenatal screening. This tension between the diagnostic and 'therapeutic' aims of such screening have been discussed elsewhere, and discourses of bonding would appear to crystallise such contradictions.

Only a handful of studies have sought to measure this relationship between ultrasound, maternal bonding, and the experience of fetal loss; however a 1992 study on this experience (Black, 1992) found that $44 \%$ of women surveyed reported that undergoing scans during pregnancy had significantly increased the sense of grief 
following a miscarriage or termination of pregnancy. Sandelowski \& Jones (1996) note that following a diagnosis of fetal abnormality, pregnant women often express the need to construct 'healing fictions' to reconfigure their experience and begin the healing process. ${ }^{195}$ It is certainly not difficult to suppose that this pain might be felt more acutely when a fetus has previously been seen alive in utero, as Sandra's devastating experience seems to suggest (see chapter three). In her ethnographic research on prenatal ultrasound, Sandelowski (1994a) found that the 'real-time' aspect of scanning encouraged parents to 'fictionalise' fetal life by attributing consciousness and volition to the movements they observed. As a result, she argues, fetal movements were frequently interpreted in terms of a fetal performance or attempt to connect with parents, for example by waving or "looking at" them (1994a:269). Similarly, Mitchell \& Georges' interview-based fieldwork notes the attribution, by both parents and sonographers, of intention and personality to fetal movement, for example in the notion that certain fetal positions denote 'shyness' or 'modesty' $(1997,1998) .{ }^{196}$ As they found, some clinicians actively encouraged pregnant women to personalise their fetuses, citing an example where a parent is reprimanded for using the term 'fetus' rather than 'baby' (1997:377).

A second and perhaps more compelling case against the wholesale institutional endorsement of fetal-maternal bonding as a legitimate aspect of prenatal care can be seen in evidence that clinicians themselves may develop affective bonds to the fetuses they image, a fact which arguably would influence the clinical impartiality of their practice. A 1992 study undertaken to determine sonographers' attitudes towards fetal imaging concluded that exposure to fetal imaging during the course of their

\footnotetext{
195 This could perhaps be theorized as another example of the unacknowledged 'moral work' undertaken by pregnant women in their engagement with prenatal screening (see Chapter four) ${ }^{196}$ Sonographers' personalization of fetuses is similarly noted by Taylor (2000).
} 
professional career led them to hold more conservative attitudes towards abortion than they had previously held. A startling $70 \%$ of respondents stated that they believed abortion of a healthy fetus should not be permitted at all, or should not be allowed beyond the 10 th week of pregnancy (Ruiz \& Murphy, 1992:273). Furthermore, 92\% of those surveyed indicated that the benefits of improved bonding made possible by providing women with detailed visual and verbal feedback during scans outweighed any possible negative consequences. Mitchell \& Georges' (1998) finding that clinicians may even express a preference for certain types of fetuses seems to provide further evidence for Franklin's contention (1991, see earlier) that practitioners sometimes view themselves, at least to some extent, as engaging in a form of personal relationship with their fetal subjects. As they note:

The compelling nature of this cyborg is especially evident when sonographers see an image they particularly like. Their posture, facial expression, and voice change as they lean closer to the screen, often tilting their heads and smiling. Sonographers may even touch, stroke, and "tickle" the on-screen image, particularly the fetal feet, and create a voice so that the fetus may "speak" to the expectant couple and communicate its "feelings". (1998:109)

Sandelowski has argued that "Technologies need stories to sell themselves and to justify their expense" (1998:9). I would suggest that increasing prominence of fetalmaternal bonding as a discourse within contemporary clinical literature can be seen to reflect a desire within the medical institution to secure a position of pioneering 'authorship' over knowledge about fetal life in general. As this discussion has suggested, the trajectory of these evolving discourses of bonding deserves both social scientific and medical scrutiny in terms of its extra-scientific form and content. 


\section{Theorising clinicians' extra-scientific uses of fetal imaging}

Previous discussion positioned clinicians' responses to and practices around fetal imaging in terms of a fetish, and considered examples of 'extra-scientific' discourses and practices arising from this view. I will now consider possible ways to theorise and account for this aspect of clinicians' practice, firstly with reference to the notion of medicine as a discipline increasingly oriented towards 'spectacular' practices, and secondly in terms of the vicarious character of the pleasure clinicians may take in fetal imaging.

\section{Prenatal ultrasound as 'spectacular medicine'}

While a significant body of social scientific work has theorised the popular embrace of fetal imaging in terms of the "cultural valorization of the visual" (Mitchell \& Georges, 1997:378), ${ }^{197}$ as a broad historical phenomenon, less has been said about the scope or nature of medicine's valuing of fetal images. In seeking to further explore the form and content of the clinical passion for prenatal imaging, I return here to Sandelowski's work, specifically her analysis of medical ways of looking as oriented towards spectacle. ${ }^{198}$ Sandelowski argues that medical caring (specifically nursing) has become more 'spectacular' since the emergence of a range of 'visualist' technologies of clinical investigation and monitoring (including ultrasound, cardiac and fetal heart rate monitoring, and CT scans). As she suggests, this preoccupation with technologically mediated data has increasingly resulted in a less intimate, more 'hands-off' mode of care in contemporary medicine (including obstetrics) and heralded a profound shift in the way the 'reality' of the fetus is both conceptualised and communicated. As she notes of nurses' practice in particular, "our concept of the

\footnotetext{
197 See for example Duden (1993) and Draper (2002).

${ }^{198}$ See in particular Sandelowski (1998) and (2000).
} 
fetus is almost wholly composed of sonograms and fetal heart traces. The nurse using a fetal monitor is arguably oriented less to the fetus per se than to the strip of paper representing the fetus" (2002:110). Such observations highlight a rather problematic 'side-effect' of medicine's wholehearted endorsement of scanning as the most authoritative source of knowledge about pregnancy. In coming to rely on this specialised technique of monitoring and measurement, rather than traditional methods for tracking fetal growth and development, ${ }^{199}$ obstetricians are recognised to be losing this set of skills. ${ }^{200}$ At the same time, midwives, who do significantly rely on these older 'hands-on' techniques, and do not themselves use ultrasound scanning in their practice, are increasingly vulnerable to being viewed as less authoritative or 'safe' in their practice, in spite of the fact that the vast majority of normal pregnancies and births are in fact conducted under their care. Irrespective of these auxiliary consequences of a spectacular approach to clinical practice, the argument that medicine's liberal use of fetal imaging simply reflects a generalised equation of technological development with the notion of clinical superiority and scientific progress is a compelling one. However I would suggest that this is not entirely sufficient to account for the distinctly extra-scientific dimension of this embrace. Petchesky (1987) argued that a 'medicalisation' thesis alone does not adequately explain the nature and scope of the popular 'market' for this technology; I would argue that this also seems to be true of medicine's enthusiasm for this practice, and the extent to which the field of fetal imaging can be seen to have "enjoyed a career spectacularly unhampered by the potential brake of clinical evaluation" (Oakley, 1984:182). In the absence of a significant amount of ethnographic research into the

\footnotetext{
${ }^{199}$ For example, palpation of the abdomen to determine fetal gestation, size and position, and intermittent abdominal measurement using a tape measure.

${ }^{200}$ See for example Wagner (1994) for a clinician's perspective on what he views as a 'de-skilling' of obstetric practitioners.
} 
views of sonographers/obstetricians themselves, and in particular the ways they themselves account for their practices and beliefs, it is only possible to make a somewhat speculative case on this issue, with reference to the small amount of existing social scientific discussion. The following discussion will briefly explore what form clinicians' enjoyment of these practices might arguably take.

\section{'Show and tell': sonography as a vicarious pleasure?}

Among the earliest qualitatively based research specifically focussing on prenatal ultrasound as both a practice and an experience, Sandelowski’s (1994a) work emphasised the different meanings and intentions attached to fetal images by clinicians and lay people. Conceiving of this distinction in terms of the different 'usecontexts' of the technology, she argued that while clinicians approached fetal imaging with an agenda of surveillance and assessment, pregnant women and their families tended to regard scans as an essentially relational encounter; specifically as an opportunity for 'appraisal', 'acquaintance' and 'care' (1994a:272). In this analysis, while clinicians viewed the imaged fetus as 'an object of scientific scrutiny' (1994a:272), parental gaze was in contrast 'communicative and even erotic, in that couples sought to interact with a love object" (1994a:272). While Sandelowski's work certainly suggests that both pregnant women and clinicians have passionately embraced prenatal scanning (as suggested earlier in this chapter), her notion of a clear distinction between the way this technology is used within medical and lay 'usecontexts' seems less resonant within the context of more recently evolved scanning practices. As the previous discussion of discourses and practices of maternal-fetal bonding would seem to suggest, medical discourses around fetal imaging (and, I would argue, prenatality in general) are increasingly extra-scientific in character, spreading into domains of interest previously outside the scope of medical enquiry. 
Nonetheless, in signalling the usefulness of framing clinicians' (and not only pregnant women's) responses to prenatal scanning in terms of desire, Sandelowski's work provided a starting point for enquiry into the extra-scientific dimension of sonographers' practice.

The bulk of subsequent social scientific research on prenatal ultrasound has tended to focus on the ways pregnant women engage with this technology, paying rather less attention to clinical practices. However, more recently, closer attention has been paid to such questions as the relationship between clinicians' practice and the broader cultural embrace of prenatal ultrasound. In particular, Taylor's work (1998, 2000, 2002) has considered clinicians' role in constituting fetal imaging as a socially oriented ritual of 'showing' rather than a strictly medical encounter. As Taylor points out, the discrepancy between the protocol and practice of prenatal ultrasound persists as a puzzling anomaly in clinicians' practice. $^{201}$ In my view, however, there is considerable scope to further explore this tension more deeply, and in particular to attempt to unpick what may be at stake for clinicians in not only 'channelling' the desire for ultrasound, but in seemingly inciting it, as earlier discussion would suggest. Such evident tensions between the social and medical tenor of clinical discourse on ultrasound seem to beg the question of, what, specifically, might be the nature of the pleasure clinicians derive from using this technology?

\footnotetext{
${ }^{201}$ As noted in chapter two, the 'non-indicated' (i.e. routine) scanning of normal pregnancies is a practice which is not endorsed by a number of influential clinical bodies in the field of obstetric medicine: most notably the American College of Obstetricians and Gynecologists (ACOG), the American Institute of Ultrasound in Medicine (AUIM), and the World Health Organisation. Nonetheless, a significant number of obstetricians appear to be in disagreement with protocols requiring conservative use of pregnancy scanning (see for example Levi \& Chervenak, 1998).
} 
Observations of clinicians' practice 'in the field' such as the work of Taylor (1998, 2002) and Mitchell and Georges $(1997,1998)$ clearly suggests that sonographers (at least within a Western setting) ${ }^{202}$ regard 'showing the baby' as among the most pleasurable aspects of their role, and often take a special interest in facilitating parents' enjoyment of scans. ${ }^{203}$ The enjoyment associated with performing scans is also noted in the clinical literature, for example as Baillie et al remark: "Many obstetricians who scan will recognise the sense of joy and excitement that many women express on seeing their baby for the first time via a real-time scan" (1997:1223). It would seem reasonable enough to assume therefore that sonographers simply enjoy performing scans because pregnant women enjoy having them. However, while the above quotation would seem to suggest that this vicarious pleasure is strictly altruistic in nature, this explanation does not seem to adequately account for the profound enthusiasm evident in the tone of much ultrasonographic literature. Mitchell \& Georges' (1997) observation that during scans, both sonographers and parents “closely resemble people admiring a baby in someone's arms" (1997:378) seems to suggest a kind of psychological investment which extends beyond the boundaries of a traditional doctor-patient relationship.

As Taylor's work suggests (2002), sonographers themselves conceive of their role as extra-scientific in nature, tending to frame their professional identity in terms of a unique, quasi-sacred form of care giving. In their privileged capacity to "show the baby', these practitioners view the significance of their work as extending well

\footnotetext{
${ }^{202}$ As their comparative research between sonographic practice in North America/Canada and Greece revealed, Greek sonographers practise very differently from their counterparts in other countries, tending to treat scans as a strictly diagnostic encounter with minimal 'extra-scientific' content.

${ }^{203}$ Taylor's work does note however that some sonographers nonetheless feel somewhat uncomfortable with the contradictory expectations attached to their role.
} 
beyond tasks of clinical measurement and diagnosis. For example, as one sonographer in Taylor's study described her job:

In this age of technology, let us not lose touch with the humanity of our job. Let us remember the tumbling fetus we image, with arms and legs fluttering in amniotic fluid, faces with big, dark eyes peering out at the excited parents. You hear the "oohs" and "ahs" at the commencement of life when the bondings of the strongest kind are initiated. This is truly what the core of our job is about ...memories, faces, beginnings - images permanently etched in the corners of our minds.

(Durbin (1997), cited in Taylor, 2002:374)

If this revelatory, privileged access to a special moment of 'showing' is such a fundamental element of sonographers' perception of their role, it is perhaps no wonder that those working in this area might be so inclined towards the enthusiastic (and liberal) use of this technology. Furthermore, as Georges (1996) suggests, women's intense gratitude towards clinicians for their role in prenatal care may also be significant in clinicians' acquiescence to popular demand for frequent scanning. As she points out, the eventual birth of a normal baby in the vast majority of pregnancies further affirms the logic of ultrasound as an emblem of medicine's authority over pregnancy care.

At the very least, it seems a case could be made for the claim that considerable enjoyment might be found in being the gatekeeper to an otherwise private, embodied experience, and in seeming to 'author' this first encounter. The notion that clinicians might through their discourse and practice assert (albeit unintentionally) a form of 'intellectual property' over individual pregnancies by dint of being the first to 'reveal' them to their pregnant patients resonates with Oakley's argument that scans have 
come to eclipse the first felt experience of fetal life $(1984,1986) .{ }^{204}$, a phenomenon described by Mitchell \& Georges (1997) as "technological quickening” (1997:373). There is certainly much evidence in the clinical literature of a general presumption that the most legitimate forms of knowledge about a pregnancy are always those held by clinicians, and not pregnant women themselves; for example, technological dating of pregnancies via ultrasound is emphasised as superior to a woman's own records, even where she is sure of the date of her last menstrual period before becoming pregnant. ${ }^{205}$ Furthermore, it is interesting to note the suggestion in such literature that women themselves may be more inclined to value technologically mediated evidence over and above their own experiences of fetal movement in acknowledging the 'realness' of their pregnancy and in forming an emotional connection with it. For example, Sedgmen et al note that:

The presentation of fetal images via ultrasound early in pregnancy before any bodily cues are experienced has superseded the earlier importance of quickening for the mother's realization of actual life inside the womb and may contribute towards stronger feelings of attachment to the unborn child and enhanced health behaviour during pregnancy.

(Sedgmen et al, 2006:245)

Within an additional excerpt from a clinical text this presumed 'redundancy' of the phenomenon of quickening appears to be taken even further; with Kurjak et al (2002) stating that "there is no question that visualization of fetal life confirms viability to parents" (Kurjak et al, 2002:62). This rather underwhelming observation brings to mind Oakley's conception of prenatal technologies as evidence of a project to 'eclipse' embodied (specifically women's) forms of knowledge.

\footnotetext{
${ }^{204}$ Historically known as 'quickening'.

${ }^{205}$ See for example Mongelli et al (1996).
} 
However clinicians' practices (and the various 'intentions' influencing them) might be theorised, it seems clear that the anomalous fusion of medical and extra-scientific expectations which characterises the phenomenon of contemporary prenatal scanning requires a kind of 'suspension of disbelief' on practitioners' part. That is, a sacrifice of logic and objectivity in the interests of enjoyment. This seems curious, given the emphasis increasingly placed on the need for an 'evidence-based' approach within healthcare, and the need for self-reflexive clinical practice. ${ }^{206}$ It might be suggested that while clinicians such as nurses and midwives are increasingly called upon to justify their practice in terms of conformity to strict protocols, maternity practitioners considered to be specialists of fetal imaging are somehow exempt from this requirement due to their privileged status as gatekeeper of the fetal image.

Sandelowski has argued that "Nurses must re-vision themselves as critical players in the selling, justification, and storying of technologies, deploying images of persons and health that re-create both" (1998:9). Perhaps this appeal for a critical eye on the form and intention of prenatal scanning practices (not least the constructed nature of the imaged fetus) might now be usefully directed towards the somewhat rarefied institution of prenatal ultrasonography.

\section{Conclusion}

For pregnant women and clinicians alike, fetal ultrasound has and will almost certainly continue to play a pivotal role in producing what is regarded as authoritative knowledge about pregnancy, and, as this chapter has shown, what we 'know' about the fetus cannot be seen to be contained within strictly medical ways of knowing. As Duden suggests, the image of the free-floating fetus has a resonance which extends far

\footnotetext{
${ }^{206}$ See also chapter six.
} 
beyond the parameters of health care, and is now irrevocably embedded within "the mental universe of our time" (1993:14). Chapters three and four signalled that pregnant women can and do sometimes engage with prenatal ultrasound on their own terms, or at least in a somewhat negotiative way. However, I would suggest that such 'uses' of this technology are nonetheless profoundly shaped and channelled by the practices which construct the fetus as a 'cultural artefact' (Taylor, 1992:67) and not simply a biological entity. This point is succinctly made by Mitchell \& Georges, who note that "Although the ultrasound apparatus may dramatically expand the sensual and cognitive apprehension of the fetus, it always does so within the constraints of dominant discursive formations" (1998:120).

The purpose of this discussion is not to question the professional intentions of contemporary sonographers in their practice, but rather to draw attention to an evident 'muddying' of roles within this work. More qualitative work in this area would be useful to shed light on the extent to which these clinicians themselves are aware of these tensions, or indeed actively participate in the extra-scientific 'role-play' which may increasingly reflect popular expectations of them. Such work would perhaps reveal that sonographers deliberately invest a new kind of 'emotional labour' in their engagement with pregnant women which they see as appropriately responsive to the 'meaning' of prenatal ultrasound in the contemporary climate.

However, this analysis would not seem to adequately recognise the ethical responsibility these practitioners nonetheless arguably carry in terms of representing this technology in a way which facilitates sufficiently informed and deliberate use by lay people. As has been argued throughout this thesis, the discourses and practices of 
clinicians do mediate, in readily identifiable ways, women's expectations around the purpose and value of pregnancy scans. Work such as Sandelowski's (1994a) and Taylor's $(1998,2002)$ suggests that the specific 'way of seeing' attached to prenatal ultrasound is a learned one; that the image of the fetus should always therefore be recognised as a significantly social construct. While as Taylor's (2002) research suggests, contemporary sonographers are at pains to secure a public perception of their role as authoritative and respectable professionals in their own right, it does not seem unreasonable to expect that such attempts should include ongoing and dispassionate scrutiny of the role this profession may play in the social construction of contemporary understandings of both fetality and maternity.

As this chapter has argued, scrutiny of the benefits (both presumed and claimed) of emerging scanning practices raise a number of questions about the nature and scope of fetal imaging as a project of contemporary prenatal care. Included among these is the concern that the flourishing of discourses of 'fetal-maternal bonding' attached to emerging technologies may signal a precarious future for women wishing to set the terms of what, for them, constitutes acceptable pregnancy care. As a good deal of feminist work around reproductive technologies has argued, the incautious use of fetal imaging has played and continues to play a key role in maintaining "the fiction of the independently viable and amaternal fetus" (Sandelowski, 1998), a notion which presents a very real challenge to women's status as the primary agents of maternity care. To return to Emily's remark (see quotation number two, head of chapter) it seems clear that clinicians, perhaps no less than pregnant women, variously draw on both medical and social 'stories' in making fetal images meaningful. In this respect, sonographers' and obstetricians' participation in (as well as broader institutional 
endorsement of) practices which both reflect and foster a 'spectacular' response to fetal imaging (Sandelowski, 1994a) would seem to constitute a largely unacknowledged aspect of the broader problem of informed consent for prenatal screening. The ways this might be meaningfully addressed within the context of clinical practice will be considered in the following chapter. 


\section{Chapter Eight: \\ Conclusion: Revisiting consent: prenatal screening as an ethical 'special case'}

Every woman hopes her baby is healthy. There are a number of screening tests available during pregnancy to look at the health of you and your baby.

(NSU, 2009)

If you ask people who are about to have a baby what they want for their child, they will often say 'a healthy baby'.

(Bioethics Council, 2007)

As this thesis has argued, representations of prenatal screening in the public domain typically paint a picture of a homogenous, unproblematic experience, whereby screening is understood as something pregnant women automatically will and indeed should choose to undergo in the interests of responsible mothering. This constitution of prenatal screening as 'what women want', that is, as both a 'choice', and as the right choice' - raises important questions about the ethics of current screening practice, casting considerable doubt on the appropriateness of the current reliance on 'uptake rates' as an index for pregnant women's acceptance of this technology.

Commenting on much earlier qualitative findings on the experience of prenatal screening, Rapp suggested that the issue of whether pregnant women 'want' screening, whether it constitutes a benefit or a burden, was ... a question to which only local and unstable responses may be given. It cannot be settled by recourse to a universal explanation, as if all women held similar interest in the problems of pregnancy, or of disabled children" (1987:114). 
In keeping with this insight, the purpose of this research has not been to make any broad claims as to whether or not women 'want' prenatal screening, but rather to explore some of the 'local responses' to this practice in the light of recent developments in screening practice, and to investigate how pregnant women might process and respond to this experience.

In light of the experiential accounts discussed here, it certainly seems to be the case that liberal notions of 'choice' in terms of the freely made selection of 'either/or' options fail to reflect the inherent complexity of the screening decisions which pregnant women confront. While pregnant women may ultimately regard choice as an ideal, it would seem that the choices they make are nonetheless often attended by considerable ambivalence. As the personal accounts of screening discussed in this thesis suggest, a healthy baby is not necessarily the exclusive goal or 'outcome' which women attach to pregnancy. and in this respect it seems necessary to recognise the extent to which the diagnostic imperatives of medicine do not always mirror the expectations of individual women. Pregnant women do not necessarily view fetal abnormality as something to avoid 'at all costs', but instead, while not welcoming such potential outcomes, they may nonetheless frame this possibility in terms of a life experience which they would somehow 'rise to' if and when this were necessary, in a way which is able to be accommodated within their 'moral biography of the future' (Scully et al, 2007). This is not to suggest that pregnant women do not seek access to the most accurate screening methods possible, or hope to be fully informed of the screening options available to them. To scrutinize the notion of prenatal screening as a tool of 'choice' is not to suggest that such technologies do not in fact offer material benefits for pregnant women. While many participants expressed some degree of 
concern around the broader social impacts of this practice, the majority held the view that prenatal screening should nonetheless be available to pregnant women. However these accounts do suggest the need to consider the extent to which the quality of the pregnancy screening experience, and in particular, the extent to which individual women feel themselves able to make decisions in line with personal values and beliefs, also appears to be extremely important for the extent to which a particular pregnancy experience is evaluated positively or negatively.

As I have argued, trends to constitute routine prenatal screening as a public health good through the implementation of routine screening necessarily position all pregnant women as 'at risk' of fetal abnormality. The version of 'choice' which emerges in this context is characterised by an array of privatised responsibilities which confront individual pregnant women with complex decisions. The shape and scope of the 'moral work' entailed in this decision-making is yet to be fully uncovered, let alone acknowledged within clinical discourse. Far from being a homogenous experience, each individual screening encounter throws up a range of possible sequelae, each requiring that decisions be made. This study has highlighted the diverse scope of this experience, which, among other things, included the necessity of evaluating the following: which currently detectable fetal abnormality might or might not be personally 'worth' a termination; the long term emotional and economic consequences of decisions for and against terminating a pregnancy; weighing the risks of invasive diagnostic tests versus the wish for a definitive result; and deciphering the personal significance of the probability-based risk estimates produced by screening tests. The 'work' attached to these complex decisions arguably sets the experience of prenatal screening apart from other scenarios where patients are required to make choices about the medical care they receive. In particular, the 
anxiety which may attend the experience of being 'at risk', or as Lupton (1999) suggests of the contemporary experience of pregnancy, embodying risk, and the responsibility of personally weighing this risk. This 'existential' dimension of translating risk as an abstraction to a personally meaningful scenario is succinctly characterised by Getz \& Kirkengen (2003:2052):

There is a vast discrepancy between the scientific term population-based risk and the social term of risk perception - the experience of being at risk. To the clinician, risk retains the character of a population-based number, but to the individual pregnant woman, the population base is one, herself, and 'one in a hundred' means that she can be the one.

As this concluding discussion will suggest, the extent to which prenatal screening may exist as an ethical 'special case' may require a rethinking of current practice which better reflects the expectations of pregnant women themselves as the presumed beneficiaries of this choice.

Whether or not programmes for prenatal screening are implemented in an official capacity in countries such as New Zealand, it seems clear that a routine approach to prenatal screening is now a firmly embedded aspect of contemporary prenatal care. Given that many participants recounted their experiences as somewhat unsatisfactory, it therefore seems worthwhile to consider how their concerns might be practically addressed within the context of existing frameworks.

\section{What do pregnant women seek in their engagement with prenatal screening?}

A key aim of the empirical component of the research was to explore how notions of choice and consent translate into the 'real life' experience of pregnant women facing screening decisions. Given that the issue of informed consent is a key concern of contemporary bioethics, it is interesting to consider how this notion might be defined 
by women themselves, and particularly, what for them would constitute a meaningful consent. As discussed in chapters three and four, dissatisfaction with the manner in which screening was presented within the clinical setting emerged as a key concern in women's accounts, with factors such as a lack of information and a casual or directive style of practitioner counselling variously felt to have undermined the possibility of informed, consensual decision-making. Where such concerns were expressed during the course of an interview, participants sometimes described what would have been, in their view, a more ideal scenario. As personalised 'definitions' of informed consent, these comments offer useful insights into ways to better tailor existing protocols to pregnant women's expectations.

For Alosina, Naomi and Sandra, being able to give an informed consent meant being made aware of the likely trajectory of the choices available to them, and included information about the known limitations of existing technologies, and whether or not support would be available. For example, Alosina described her preference for the appropriate presentation of screening:

What I would have liked is a really fair laying out of the options, so that it wasn't all weighted to (it's) a choice between having the choice or not... and then once you have the test, either getting good results or bad, you know, like putting them in those choices? And normally I'm quite trusting of the medical profession, you know, I believe in Western medicine. But, I think because you're quite vulnerable when you're about to have a child, there is a need for a much more careful offering of choices, yeah? I think the government, the medical profession have a responsibility to give full information and unbiased information.

As her comments suggest, the presentation of screening in terms of dichotomies of good outcomes versus bad outcomes, acceptance versus refusal, presents an unreasonable obstacle to informed decision-making, and to the possibility of tailoring 
decisions to individual preferences. Naomi described her ideal by describing how she herself would inform a pregnant friend about prenatal screening:

I think I would just try to let them know that what comes out of it is a risk factor, then once you know that risk factor then you would need to make a decision as to whether to take it further or not. Just to allude to the fact that there might be some more important decisions to go on, and that if you don't want to know, then don't have the scan. You know, like represent the option as an option not to have it at all if lack of information would actually make you feel better than information.

Similar to Naomi and Alosina, Eva emphasised the need for practitioners to qualify the choice available, and to be explicit about the various possible outcomes:

You have these choices now, you may choose this, but if you choose this then you might have other choices to make. You may choose not to, and in choosing that, this might be the outcome. So you've got the whole picture. Yeah, I think that would be better.

Each of these comments suggests the significance to women that clinicians recognise the complexity of these decisions, and the extent to which they are not experienced as 'black or white'. Furthermore, it seems clear that the urgency of clinical imperatives to make a decision has less traction within this perspective than the importance that any decision which is made is felt to be personally acceptable. This would seem to reiterate the argument made in chapter four that individuals facing complex moral decisions may prioritise the personal acceptability of choices made over the 'correctness' of the decision in the interests of a personally acceptable "moral biography of the future" (Scully et al, 2007:217).

These accounts also underscore the extent to which individual women may view the choice not to screen somewhat differently from clinicians, who, while theoretically endorsing the notion of 'informed refusal' as the corollary of the principle of informed consent, nonetheless work within institutional and policy frameworks 
informed by broader directives, which as chapters three and six suggest, ultimately constitute 'non uptake' as an epidemiological challenge. In contrast, it appears individual women may view the choice to refuse screening as a highly deliberative decision, and regard the medical sanctioning of this choice as a fundamental requirement of individual autonomy and authentic consent. As Naomi's account suggested, the protection of the 'right not to know' stood, for her, as a 'bottom line' measure of ethical practice.

In seeking to address women's concerns about consent, it therefore seems worthwhile to consider ways to formalise the principle of informed refusal and to materialise this ideal within the context of day to day practice. The metaphor of the 'pathway' is often used in contemporary public health discourse to describe the processes involved in patients' engagement with the health system, the pathway generally being presented as a straightforward trajectory between screening or testing, diagnosis, and treatment. The notion of this process as a staged, orderly progression broken down into discrete steps doubtless is intended to reassure patients that they are able (and to some extent expected) to be involved in choosing the direction of their care. I would suggest that within the New Zealand context, the option of refusing prenatal screening has not, until very recently, been recognised in terms of a legitimate step on the prenatal screening pathway to the extent that many other countries have done. ${ }^{207}$ However, this may now be changing in light of the recent review of screening for Down syndrome, and the statement below recently posted on the website of the NSU suggests a move

\footnotetext{
${ }^{207}$ For example, the 'screening pathway' outlined in the 2007 NSU report on Down syndrome screening in New Zealand concludes with the discussion of 'intervention options' (see page 10). In contrast, in the 2004 National Health Service handbook for staff on Down syndrome screening, a section entitled "Pathways through the screening process" clearly and neutrally presents the decision to decline screening as among the possible outcomes to be expected by clinicians. Similarly, as noted in chapter five, the Dutch government explicitly enforces this option within maternal health policy.
} 
towards official recognition of the need to formally and publicly sanction the choice not to screen as a legitimate option for pregnant women in New Zealand.

Only you can make the decision about whether to have testing or not. Some women want to know about possible conditions and some do not. Your Lead Maternity Carer (LMC) or GP can provide you with resources and details of relevant organisations for additional help with your decision. Women who choose not to be screened will be supported in their decision.

(NSU, 2009)

While the extent to which this support will translate into material resources and social services for families of disabled children remains to be seen, it seems likely this would be welcomed by many participants in this research as evidence of a shift towards a closer alignment of medical and lay expectations of choice. This very recent official endorsement of informed refusal in New Zealand may be a useful starting point for addressing concerns about the lack of sanctioned alternatives to termination of pregnancy where fetal abnormality is found. I will now consider a number of other potential changes to current practice which emerged from participants during the course of the interviews.

\section{Facilitating informed consent: some pragmatic suggestions}

Concerns around the sufficiency of information about screening, and the appropriate 'delivery' of this information were key themes in women's accounts, and as suggested in previous chapters the contribution of an 'informational deficit' among lay people is now widely recognised within bioethics literature as a significant obstacle to informed consent. Participants had rarely been given written information by their caregivers, and noted that they had often relied on information which they sourced themselves from pregnancy books or the internet. Therefore, the development and mandatory provision by practitioners of written information about prenatal screening would seem to be a logical starting point for addressing this concern. This approach would seem 
likely to be particularly fruitful within the New Zealand context, given recent evidence of significant deficits in practitioner knowledge. In light of the historic lack of availability of nationally consistent patient information literature, the collaboration of different practitioner types (i.e. midwives, obstetricians and GPs) in the development of this material would be ideal.

However, as chapter five suggests, reliance on the provision of written information alone far from guarantees informed decision-making among patients and may in fact be counterproductive, reproducing a bureaucratic, 'procedural' model of consent which does not reflect a genuine commitment to facilitating informed decisionmaking. Not only do patient information leaflets often fail to outline the limitations of available screening methods (such as rates of false-positive and false-negative diagnoses), as Milligan (2004) suggests, this material also cannot be relied upon to clearly spell out termination of pregnancy as the most frequent 'endpoint' of the diagnosis of fetal abnormality. While it appears pregnant women would welcome the provision of unbiased, standardised written information, the accounts of participants in this research also suggests the significance of the context of decision-making for facilitating informed consent, and not simply the informational aspect of this process. This will be considered now.

\section{Recognising the significance of time and place as contexts of decision-making}

The accounts discussed in chapter four signalled the processual character of screening decisions, and underscored the significance of time for establishing a sufficient degree of 'moral space' for individual women to reflect on the options available, and ultimately to reach a personally acceptable decision. It seem clear that the process of reaching this decision is often experienced as a 'high stakes' and highly engrossing 
process, as the following remarks by Kerry suggest: "Once I'd decided not to have it, the amnio, I felt fine, I thought this will be ok. But the period when I was deciding not to have it, it occupied a lot of my headspace". More broadly, participants accounts highlighted a sense of time pressure as a common experience associated with screening, and a corresponding loss of control, a feeling conveyed through metaphors of powerlessness, for example being "on a conveyor belt" (Sandra), "on the track" (Patricia), being "caught in a trap" and "in the system"(Anna). Such comments suggest a need to rethink current practice in a way which recognises the extent to which protocols of informed consent ought to recognise sufficient time, as well as sufficient information as an ethical requirement.

A number of women commented on the need for counselling and discussion prior to screening decisions, suggesting that the timing of such counselling played a role in

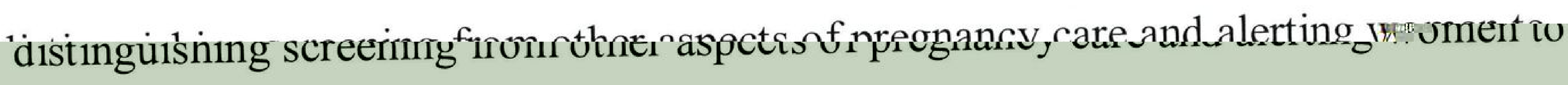
the fact that consent was actually being sought. For example, as Alosina commented:

I mean I think there needs to be much more careful counselling before the test, so that it's not like just another urine sample, or just another scan. But say "look, this could really affect your decision about whether to keep the child or not, you know?"

For Sandra the provision of screening information would ideally occur early in pregnancy, allowing private hypothetical consideration of various screening options and discussion with family members: 
And then you've got time too in those early stages to talk about it with a partner or family or whatever and say 'ok, shall we?', you know...'here are the things', or, 'I'm in my mid-forties, we should think about this but this is what it can show and if it did show Downs, you know, what would we do?' And just having that opportunity to think it all through.

Portia and Sandra also emphasised the need for practitioners to encourage women to consider possible outcomes prior to undergoing screening, and particularly to counsel women about scenarios involving negative screening results, including information about the time frame and method for termination of pregnancies.

However, structuring time for deliberation and discussion with families into existing screening "pathways' may be difficult to achieve in the context of current practice, given the increasing emphasis on first trimester screening (i.e.NTS). As noted in previous chapters, many women have only attended one prenatal visit (if any) by the time NTS is due to take place, with the resulting expectation appearing to be that the decision whether or not to have screening will occur within the context of a single prenatal visit in order that a referral for the scan can be organised. Clearly, this would seem far from ideal and suggests the need to rethink the sequencing of the informational and reflective aspect of current screening practice. Perhaps it may be preferable to extract this element from the context of the generic prenatal visit and consider a more suitable forum for pre-screening information and counselling? Milligan (2004) has suggested that the prenatal visit is not an appropriate educative opportunity for pregnant women. As she argues, within this environment, practical contingencies such as limitation of time and resources undermine the extent to which 
both pregnant women and practitioners are able to engage in what she describes as the “dynamic process of dialogue, collaboration and negotiation" required to facilitate informed decision-making (2004:12). As an alternative, she proposes that this "ethical encounter" might better take place within a setting similar to an antenatal class, as an already-familiar educative forum, a model which she suggests would help keep “moral-reflective spaces open within institutional frameworks while each woman's emotional, physical and knowledge status evolves" (2004:12).

Given that to date few practical solutions have been put forward to address the problematic of informed consent for prenatal screening, Milligan's suggestion seems worth consideration. However, her conception of this forum as ideally taking place within the institutional framework of prenatal care, (presumably still within a hospital setting, and run by maternity staff) ${ }^{208}$ seems somewhat problematic. As this thesis suggests, "institutional frameworks" and their objectives are sometimes radically at odds with lay worldviews and ideals of 'health' and 'normality'. Furthermore, if institutional support is the most significant determinant for women's uptake of prenatal screening (Press \& Browner, 1997), a forum which takes place within the hospital setting may not offer a suitably 'neutral' context. .A more appropriate model might already exist within the New Zealand setting, where antenatal classes usually take place independently of the clinical setting (often being led by 'lay' childbirth educators, rather than clinicians, and taking place in community centres and church halls). Such sessions usually conclude with an opportunity for group members to mingle and share experiences over a cup of tea or coffee. This environment might offer a more relaxed forum for receiving and processing neutral (hopefully)

\footnotetext{
208 This may reflect the fact that in Australia prenatal education classes typically take place under the auspices of clinical services, unlike in New Zealand where they are generally independently run.
} 
information about all possible 'pathways', and it may be possible to 'add on' a module on the topic of screening to topics already covered in this class. While this may not directly benefit attendees who have already made their screening decisions (although they may benefit in subsequent pregnancies), it seems likely that establishing a "moral-reflective space" about prenatal screening within this context would gradually lead to a broader community awareness that such decisions are now part of the experience of pregnancy.

Whatever the logistics of doing so may be, it seems reasonable to argue that, at a minimum, pregnant women should be given the opportunity to deliberate on their decision outside the context of a single prenatal visit. As Garcia et al (2008) suggest, women's screening choices are usually made in the context of relational ties, that is, with reference to the needs and wishes of other family members. As they argue, "autonomy cannot be considered independent of the relational network of moral agents" (2008b:119). Thus it seems important that screening pathways factor in sufficient time and 'space' for women to talk these decisions through (if they wish) with family before a decision is expected.

Measures such as those discussed above might go some way to reconfiguring consent in light of women's expectations, and encouraging clinicians to recognise the nature of consent, as Corrigan suggests, "as a process as opposed to a 'once and for all act"” $(2003: 786)$.

\section{Practitioners' role as representational agents of prenatal screening: the need for professional training and support}

Given evidence of this expectation that screening decisions be made, more or less, conterminously with the provision of information during a single prenatal visit, the 
stakes would seem to be extremely high for individual practitioners to provide a sufficiently in-depth, balanced overview of the options available. As suggested in previous chapters, this requirement is further problematised both by evidently poor levels of clinical knowledge around the appropriate use of specific screening methods (particularly within New Zealand), and by the tendency of some clinicians towards a directive approach. This raises the question of what exactly is the nature of clinicians' role in this encounter, and what 'extra-medical' skills might be called for.

As Portia suggested, more is required than the simple transference of information, as in her view the role of the clinician was also to initiate deliberation in the first instance, and indeed to draw this out. As she remarked:

the medical professional needs to be sufficiently skilled, then, to facilitate the conversation that that person is then interested to say well what are you looking for and what might that mean? They're able to carry it on, yeah, I mean it's a tricky thing but I think they need to have those skills.

What such comments would appear to suggest then is an expectation that practitioners themselves play a role in establishing the conditions for personal ethical deliberations by drawing on a particular communicative approach. The notion that clinicians might have an ethical obligation to instrumentalise rather than direct women's decisionmaking processes in this way is a far cry from traditional 'paternalistic' models of doctor-patient interaction. Nonetheless, recent years have seen calls from within clinical circles to reform medical education towards a far greater emphasis on interpersonal and communicative skills, including in the area of prenatal care (Marteau, 1995). Frank (1995) and Charon (2001a, 2001b) have suggested that this might be achieved through placing greater emphasis on the value of ethnographic accounts of healthcare experience within medical training. As Charon suggests, 
reflecting on patient narratives encourages a model of empathetic engagement which not only has the potential to rebalance the power dynamics of the clinician-patient relationship, but also may lead to broader benefits in terms of opening up ethical dialogue between the medical community and the general public. As she suggests, such an approach raises the possibility of a democratic rejuvenation of bioethics in opening the way for 'collective discourse' around the direction and content of contemporary health care (2001a:1900). A further benefit of this approach may be that clinicians become more self-reflexive, and attuned to the embeddedness of their own assumptions and practices within medical culture as one among a number of possible perspectives on what may constitute a 'valuable' or valued healthcare experience. With respect to sonographers specifically, ethical practice would seem to require a sufficiently self-reflexive approach, and a need to be mindful of the extent to which engaging in 'social' narratives around fetality and maternity may problematise consent. As chapters three and four showed, pregnant women do not necessarily equate genetic abnormality with ill health, as is suggested by Anna and Kiri's views of their children as 'healthy' and doing well developmentally. Such perspectives have obvious significance for clinicians in their role of informing pregnant women about conditions such as Down syndrome, and the evident tendency to either leave out such information entirely, or, alternatively, as in Kiri's case, to present the condition in terms of a 'worst case scenario', an approach which she viewed as a significant barrier to women's informed choice;

She's given information alright, but it's professional information. And it makes me cringe what they'll be told. Because it will be all negative stuff. It will be absolutely negative. I mean when (child's name) was diagnosed on the first day, you would think that this child would do absolutely nothing for the rest of his life. 
Given evidence of clinicians' own ambivalence about their competence in facilitating informed choice as discussed in chapter five, professional support and education in this area, perhaps in the form of a specific training 'module' for maternity practitioners and sonographers on counselling women about prenatal screening, would seem a valuable aspect of clinical training.

\section{Addressing the normative significance of ultrasound}

Chapter seven considered the role of fetal imaging practices in the social construction of prenatal screening as a practice of reassurance and pleasure, rather than as a strictly diagnostic event. As was suggested, the 'social' evaluations of the practice of ultrasound have broader implications in terms of the popular view of prenatal screening more generally, raising questions about the extent to which acceptance of ultrasound may be construed in terms of 'consent'. Unlike a number of other screening methods, prenatal scanning has not historically been subject to a formal requirement for consent, but instead assumes consent unless otherwise indicated. This model of consent effectively requires women to actively 'opt out' of ultrasound screening, a decision which as I have argued runs counter to an array of cultural imperatives around responsible motherhood, the value of medical technology, and the authoritative and spectacular appeal of 'the visual'.

Given the extent to which ultrasound now appears to be popularly valued and sought after as a 'social' experience rather than a strictly diagnostic opportunity, it seems clinicians and policy makers would face a difficult task in attempting to align medical and social expectations of the use of this technology. Nonetheless, I would argue that this present tension in expectations around the use of this technology care would usefully be mitigated (at least to some extent) through formalising current requirements for consent. One approach would be simply to require a documented 
consent from women wishing to undergo ultrasound. A benefit of this approach might be that, as with formalised pre-screening counselling, the expectation of active decision-making is more clearly 'sign-posted', which may encourage women who may not otherwise have done so to weigh the benefits as well as the potential costs of adding a visual dimension to their pregnancy. This may not necessarily require a written consent, but may just require evidence (such as a ticked box stamped within the woman's prenatal notes) that the issue has at least been raised, with pros and cons at least minimally discussed.

The requirement for a written consent might therefore be useful in creating a 'space' for deliberation and may go some way to addressing the concern that where ultrasound is routinely available, pregnant women tend to 'go with the flow' (Press \& Browner,1997) rather than making a deliberate decision to accept or decline. This approach might also be useful in reversing popular assumptions of the equation of the availability of this technology with clinical endorsement per se.

A second, and potentially more controversial measure to address the question of consent for ultrasound would be for medicine to respond to the 'market' for nondiagnostic scanning. It is clear that for many women, ultrasound scans constitute a much anticipated, pleasurable aspect of contemporary pregnancy care, and that 'seeing the baby' (Harris et al, 2004) constitutes a personally and culturally valued experience. As suggested in the accounts of Emily and Melissa (chapter three), scans may be viewed as innately valuable for the enjoyment attached to the experience, whether or not diagnostic information is sought. The extent to which this apparent separating out of the pleasurable and diagnostic expectations attached to scans 
appears to be viewed by women themselves as unproblematic may signal another practical way to address the problem of informed consent for prenatal ultrasound. Given the apparent proclivity for this kind of conceptual 'bracketing' of the various purposes of scanning among pregnant women, might not this distinction also be usefully marked and responded to within the context of clinical practice? As chapter seven notes, non-diagnostic scanning is already available in the commercial sector, and while to date this practice is widely condemned within clinical circles as frivolous and 'unsafe', it may in fact be the case that recognising the popular valuing of 'social' scans might offer the obstetric/sonographic community a new way to address the problem of informed consent for prenatal screening. Offering pregnant women the choice to determine the terms of their engagement with ultrasound - as either a diagnostic or non-diagnostic encounter - might very well result in increases in levels of patient awareness, if only through drawing attention to this distinction in the first instance. In a rare example of clinical endorsement for this approach, Baillie and Hewison (1999) have argued for 'selective consent' for prenatal screening as a way to better reflect patient expectations and wishes, and to acknowledge the extent to which the wish to avoid clinical data may constitute an active and informed decision. As they suggest:

Selective consent can be easily accommodated by the early pregnancy scan because monitoring functions (assessing gestation and viability, and identifying multiple pregnancies) are distinct from its screening functions. Therefore, couples who wish to decline screening for chromosomal abnormalities do not have to forgo "seeing the baby" or accurately dating the pregnancy. The data suggest that an increasing, albeit small, percentage of women who have ultrasound scans are making an informed choice to selectively decline screening for chromosomal abnormalities. It may be that the way forward in facilitating informed choice for ultrasound screening is to separate "scanning" (to monitor the pregnancy) from "screening" (for fetal abnormality).

(Baillie \& Hewison, 1999:805). 
Here, non-diagnostic scans are still conceived of in terms of a 'medical' event (i.e. for the purposes of fetal monitoring), however a clear distinction is nonetheless drawn between this practice and 'screening'. Such an approach may be useful in reaching a more satisfactory 'middle ground' between medical and popular expectations of the use of prenatal ultrasound. While sonographers may be professionally uncomfortable with any requirement to undertake strictly 'social' scans, offering scans which while non-diagnostic are nonetheless oriented towards fetal monitoring, seems likely to be more consistent with clinicians' conception of their role as primarily therapeutic, rather than as providing a consumer 'service'. Doubtless some sonographers would be uncomfortable with the requirement that they withhold diagnoses of abnormality, particularly within the increasingly litigious environment of contemporary obstetrics, however this might be dealt with by the requirement that consumers of this form of scanning sign a waiver releasing the sonographer from diagnostic liability; an approach already taken within the context of 'keepsake' scanning enterprises in the USA (Voelker, 2005).

This separation of 'scanning' and 'screening' in the use of prenatal ultrasound does not require pregnant women to relinquish their appreciation of the scanning experience as a "meaningful social ritual" (Mitchell, 2004:228) but also offers a clinically sanctioned 'way out' from the moral work associated with diagnostic information. Whatever the professional response to such a proposal might be, as chapter seven suggests, women's valuing of scans and increasing willingness to pursue these outside the context of the traditional doctor/patient relationship signals the need for clinical bodies to recognise that, whether they would wish it or not, a 
popular shift away from deference to medical authority over pregnancy is already taking place.

\section{The significance of public engagement: participants' views}

Drawing on participants' responses to questions on the ethics of current screening practice, the final section of this discussion considers the significance of public involvement in the development of prenatal screening policy, and anticipates the likely shape of processes of public engagement in bioethics decisions in New Zealand.

An auxiliary aim of the empirical aspect of this thesis related to the question of lay expectations of bioethics, and in particular the value of public involvement. While the responses generated from this line of questioning did not form a key focus of the data analysis for this thesis, this material offers some interesting preliminary insights into how pregnant women in New Zealand may conceptualise the ideal functioning of mechanisms intended to regulate ethical practice.

As suggested in chapter five, alongside the protocol of informed consent, processes of public consultation are increasingly heralded in the bioethics domain as a mechanism for assuring the ethical integrity of healthcare provision (particularly within the area of new genetic technologies). This view of public consultation as a requirement for ethical practice was also broadly reflected within participants' accounts in this research. It is interesting to note that all but two participants indicated that they regarded prenatal screening as a matter for public ethical debate, and not only as a private concern. Public involvement in decisions around the use and development of screening technologies in this area appeared to be viewed as an important ethical 
safeguard, and such accounts often emphasised the importance of diverse

representation in ethical processes, which included the views of 'ordinary people'.

Kerry's remark is typical of this view:

Well that's kind of why I was interested in the research really because I think there needs to be input from people across, right across, you know, society. Not just the doctors and the medical people and the researchers, but also from the public because I think there's quite a big sort of ethical thing here. I should also say I'm not religious in any way...so this is not from a sort of firmly entrenched religious perspective or anything, it's just you know sort of a feeling that I have that there needs to be a lot of consultation, we have to be really careful where we go with it.

For Alosina, it was very important that women's experience should be part of this

debate, or indeed constitute the starting point for broader public deliberation:

Well I think research like yours helps, you know, it helps. One, you're working with subjects, you're working with people who have the experience of tests like this, so it helps people move from their experience, from their raw experience to another kind of level, and kind of process their experience. And that's important because otherwise it's just an experience. And for it to move to a public debate, for us to get a public debate, people have to move from that experience to kind of critically thinking about well what did that experience mean? And so that's one thing. And I think research helps feed the dialogue and the debate, yeah? So one person's question has the ripple effect of getting more people to ask questions.

Similarly, Emily emphasised the importance of broader debate, and the significance of experiential accounts as 'expert' commentary within this context: As she remarked:

"See, it's only those that have walked the road that really know the ins and outs and have faced the issues". These and similar comments made by participants about the value of public engagement in ethics debates may suggest a broader reluctance among pregnant women to privately and exclusively bear ethical responsibility for the dilemmas raised by screening practices. Thus it may be the case that while liberal notions of prenatal screening as a 'choice' emphasise individual rights and freedoms, 
pregnant women themselves, while valuing such rights, nonetheless also conceive of this issue as a matter of public interest.

While, as noted in chapter three, a majority of participants held a 'pro-choice' view of abortion, many appeared to find the issue of targeted screening for specific conditions such as Down syndrome ethically troublesome, viewing it as a practice requiring a broader social mandate. For example, as Naomi commented:

Well that's a question for everybody, about whether we're trying to eliminate some conditions from our society, whether we're trying to stop them costing us so much money, that would be another way of looking at it, or whether we're just trying to give people the option as to how they want to live their lives, individuals the option as to how they want to live their lives.

While clearly signalling an expectation of public consultation in the development of screening policy, participants' accounts were ambivalent about the value of ethical 'debate' per se on this issue, suggesting that this approach would be unlikely to represent an adequate diversity of concerns. As Emily remarked, “...the trouble with public debate is you get the loud strong voices, and the quiet ones, the people that don't have the skill to speak publicly, are not always heard". Ruby held similar concerns, suggesting that traditional processes of public consultation would likely produce simplistic conclusions, and fail to reflect the complexity of what is actually 'at stake' in prenatal screening decisions:

Say if you take it to a public sort of debate, I don't know how useful it will be. I think sometimes when things become opened up to public debate the questions just get turned into a very simple yes/no, and they can miss the major point, it will get high-jacked by one group or another, it can cause rifts in society and perhaps you won't end up with results that are any better than sort of a softer approach.

Ruby's suggestion of a need for a 'softer approach' to consultation on prenatal screening, as one which recognises the complexity of prenatal screening decisions, 
brings me back to the value of subjective accounts as a key 'ethical resource' for the development of ethically responsive health policy in this area.

\section{Public engagement in bioethics in New Zealand: an uncertain future?}

It is not within the scope of this thesis to elaborate on this data, however the brief discussion above offers some preliminary insights into the extent to which pregnant women in New Zealand may regard the opportunity for lay involvement to be an important requirement for ethical practice in the provision of prenatal screening services. As chapter six suggested, to date within New Zealand there appears to have been minimal clinical interest in aligning prenatal screening policy with public attitudes toward the value and appropriate scope of this practice. No public consultation was undertaken during the course of the recent review of Down syndrome screening, and experiential studies were not cited in literature reviews undertaken for this document.

While the outcome of the review has led to a number of measurable improvements in prenatal screening in New Zealand (chiefly in terms of the increased reliability of methods now being introduced) these changes are clearly less a response to 'consumer demand' than concerns within the clinical community, and indeed broader epidemiological imperatives. What scope, then, exists for public input into the value of this now 'everyday' practice? Given the presentation of routine pregnancy screening as a moot point within the most recent public consultation on methods of 'pre-birth testing, the assumption appears to be that no such consultation was, or ever will be required. 
Indeed, more recent developments in the bioethics domain in New Zealand suggest the likelihood of a diminishing political commitment to processes of public engagement. In March 2009, Toi te taiao/the Bioethics Council was perfunctorily disestablished by the recently elected National-led government. Housed within the Ministry for the Environment, the Council had been established in 2002 on the recommendation of the Royal Commission on Genetic Modification. Following the receipt of over nine thousand public submissions expressing concern with the cultural, ethical and spiritual implications of genetic engineering, the Royal Commission noted that at that time no suitable body existed in New Zealand to respond to these concerns (Pennington, 2005). The terms of reference for the newly established Council expressed a clear commitment to fostering processes of public engagement, being to "develop and implement [other] strategies to involve, communicate and consult with the public and other stakeholders about the ethical, cultural and spiritual dimensions of biotechnology. ${ }^{209}$ These strategies will contribute to the building of trust between the public and various stakeholders" (Bioethics Council, 2007). In addition to the “who gets born' project, the Council's website profiled other contemporary bioethics research recently undertaken, including the production of reports on xenotransplantation and ongoing research on the use of human embryos, as well as a list of links to other sites of ethical interest. As such, the Council's website arguably constituted the sole official public bioethics resource in New Zealand.

In a March 2009 press release, Minister for the Environment Nick Smith commented that the committee duplicated work undertaken by other agencies and that the Government preferred to "invest in frontline services that really matter for New

${ }^{209}$ See appendix 16. 
Zealanders not expensive slogans" (Beehive.govt.nz, 2009). Such remarks would seem to suggest a view that the public have little interest in involvement in decisions around biotechnologies, that such processes are not something that 'really matter' to ordinary people. The Council's website is no longer accessible on the internet (having been recently archived off-line in the National Library) and similarly this measure indicates that fostering greater public access to bioethics information may be a low priority within the current political climate in New Zealand. This evident shift away from previous attempts to 'democratise' public policy around health technologies would seem to set New Zealand apart from a broad international trend towards greater rather than fewer opportunities for public involvement in bio scientific debates, in recognition of growing public mistrust in science (Du Plessis, 2003). In spite of the putative shortcomings of the consultative processes undertaken by the Bioethics council, ${ }^{210}$ this body nonetheless provided a platform for public engagement, not least through 'popularising' notions such as 'public deliberation' in the first instance. In the absence of this platform, it remains to be seen how the current government intends to 'make good' on any future claim that policy on the use of emerging biotechnologies is responsive to public 'needs' or wishes. Given the rapid development of prenatal screening techniques within the last decade, it therefore seems reasonable to predict that pregnant women in New Zealand will confront an increasingly complex array of decisions, and that such decisions will likely continue to be made "in the slipstream of technological advances" (Beck-Gernsheim, 2002:93), as the private moral responsibility of individual women.

\footnotetext{
${ }^{210}$ See chapter six. See also Jones et al (2009) for a critique of the decision-making framework utilized in this project.
} 


\section{Concluding comments on the value of the research}

Cunningham-Burley and Kerr have argued that where particular 'social' evaluations of screening technologies are excluded from mainstream discourse, assumptions of "an essentialist distinction between objective science and other knowledge and practice" will prevail (1999:648) In presenting a 'snapshot' of subjective accounts of prenatal screening in New Zealand, this thesis has similarly argued for the need to assert the significance, both within medical discourse and more broadly within the public domain, of prenatal screening as a social phenomenon, as an experience, and not only as a practice. While prenatal screening may indeed be regarded as an 'everyday' experience, it is nonetheless clearly a highly complex phenomenon which straddles many registers of 'knowledge' and experience. Ethical practice requires that we do not retreat from this complexity, or seek to apply a 'one size fits all' response. Instead, this response would ideally anticipate and accommodate a diversity of expectations, and express a pragmatic commitment to informed consent as an ethical ideal.

Therefore, in light of the historic and the current absence of public engagement on this issue in New Zealand, it seems important that experientially-based evaluations of this practice, such as those within this research, come to be utilised by policy makers as a resource with which to direct ethical practice and policy development in this area. As I have argued, such policy ought to recognise the potential for variance between medical and lay understandings of prenatal screening, as well as between notions of health, disability and 'good outcomes'. This particular finding suggests the value of further local research on health beliefs and conceptualisations of 'genetic health', including investigation of such attitudes per ethnicity. To date very little research has been undertaken in the area of Maori women's experiences of maternity care in New 
Zealand, and qualitative studies relating to health beliefs around pregnancy and birth would be likely to provide rich insights of considerable sociological and ethical interest.

The findings of this research, then, suggest a need for further local, broad-based inquiry into the experience of prenatal screening relative to recently-developed and currently emerging techniques such as NTS and PGD, in order that the implementation of any future screening innovations can be seen to reflect both consumer expectation and demonstrated clinical 'need'. 


\section{Appendix 1}

Glossary of clinical terms ${ }^{211}$

\section{Amniocentesis}

An invasive procedure involving the withdrawal of amniotic fluid from the uterus of a pregnant woman via a needle. Fetal cells contained within the fluid and analysed for genetic abnormality

\section{Breech presentation}

The fetus lies with the buttocks entering the pelvis first. Ultrasound is used to confirm a suspected breech presentation. Babies in breech position are increasingly delivered by planned caesarean section.

\section{Chorionic Villus Sampling (CVS)}

An invasive procedure involving removal of a sample of placental tissue for genetic analysis of fetal cells.

\section{Congenital}

Present at birth. A congenital condition may or may not be genetic in origin. Such conditions may be detected prenatally, at birth, or sometimes years after birth.

\section{Cystic fibrosis}

A genetic disorder which causes chronic lung disease and intestinal problems. Incidence is approximately 1:2500 pregnancies.

\section{Diagnosis}

Definitive (genetic) identification of a disease or condition

\section{Diagnostic test}

A genetic test which definitively indicates the presence or absence of a specific condition (for example, amniocentesis and CVS)

\section{Down syndrome}

A disorder caused by the presence of an extra chromosome 21 (i.e. three, instead of two). The syndrome may vary in severity, but is usually associated with mental deficits as well as range of physical problems. Incidence is approximately 1:600-700 pregnancies. Sometimes also referred to as 'Down's' syndrome.

\section{False-negative result}

\footnotetext{
${ }^{211}$ Adapted from National Health Service (UK) (2004), 'National Down's syndrome screening programme for England: A handbook for staff' and Medforth et al (2006), 'Oxford Handbook of Midwifery'.
} 
A screening result which has failed to identify the presence of a condition such as Down syndrome in a specific fetus.

\section{False-positive result}

A screening result which has indicated the presence of a condition such as Down syndrome in a specific fetus, where the fetus is unaffected by that condition.

\section{First trimester of pregnancy}

The period of the first 12 weeks of pregnancy.

\section{Gestation}

A weekly measure of the current duration of a particular pregnancy

\section{Induction of labour}

When labour is initiated artificially, usually where a pregnancy is considered to be post-term and/or showing evidence of fetal compromise.

\section{In vitro fertilisation}

A method of assisted fertility involving the artificial fertilisation of ovarian egg within the laboratory setting.

\section{Marker}

A clinical indicator for the presence of a particular condition.

For example, an increased area of fluid on the fetal neck is used as a clinical marker for Down syndrome (see also NTS).

\section{Maternal serum screening}

A maternal blood test which measures levels of specific hormones and proteins in order to identify pregnancies with an increased risk of conditions such as Down syndrome and spina bifida. Where the result indicates an increased risk, follow up screening is usually offered (i.e. ultrasound and/or amniocentesis). This test (also referred to as MSS2) has recently become widely available in New Zealand to women during the second trimester of pregnancy as an adjunct to NTS screening in the first trimester.

\section{Nuchal}

Referring to the (fetal) neck.

\section{Nuchal translucency scanning (NTS)}

An ultrasound scan undertaken in the first trimester of pregnancy to measure the depth of fluid (the fluid appearing as a translucent area on ultrasound) at the back of the neck of the fetus. An increased amount of fluid may indicate the presence of Down syndrome, or another condition.

\section{Polycystic Kidney Disease}

A genetic disorder of varying severity, associated with kidney and pulmonary problems. May be evident prenatally or at birth, or present later in life.

\section{Post-term pregnancy}


Clinical protocols vary, however a pregnancy is usually considered to be post-term when it has continued beyond 41 weeks duration.

\section{Prenatal screening/Antenatal screening}

These terms are now used interchangeably. Refers to a range of evaluations of maternal wellbeing during pregnancy (including measurement of blood pressure and iron levels), as well as screening specifically to detect fetal abnormality.

\section{Prenatal diagnosis}

Invasive tests (including amniocentesis, CVS and PGD) used in pregnancy to definitively determine the chromosomal or genetic profile of the fetus.

\section{Preimplantation Genetic Diagnosis (PGD)}

A recently developed diagnostic genetic test involving the removal and testing of embryonic cells prior to implantation of the embryo in the uterus. Occurs only within the context of pregnancies created through in vitro fertilisation (IVF).

\section{Second trimester of pregnancy}

The period from the beginning of the $13^{\text {th }}$ week of pregnancy to the end of the $28^{\text {th }}$ week.

\section{Sensitivity}

Detection rate

\section{Screening}

As distinct from diagnostic tests, screening techniques are used to identify indications or 'markers' which are suggestive of abnormality, and are not considered clinically diagnostic. Currently available methods of prenatal screening include NTS, maternal serum screening, and 'opportunistic' findings during ultrasound scans for other purposes (such as assessment of fetal growth and position).

\section{Specificity}

Refers to the capacity of a particular screening method to accurately distinguish between a variety of possible findings.

\section{Spina bifida}

A birth defect caused by incomplete closure of the neural tube/spinal column.

\section{Third trimester of pregnancy}

The period from the beginning of the $29^{\text {th }}$ week of pregnancy until the onset of labour.

\section{Termination of pregnancy (TOP)}

The medical expulsion or extraction of a fetus from the uterus.

\section{'Triple test'}

A form of prenatal screening combining the measurement of specific hormones or proteins in maternal blood associated with fetal abnormality, with maternal age to predict the likelihood of the presence of specific fetal conditions. 


\section{Ultrasound scanning}

High frequency sound waves which are turned into electrical signals, producing images on a screen.

\section{Uptake rate}

The rate of women out of the pregnant population who have screening. 


\section{Appendix 2}

List of Abbreviations

GP

IVF

MSS2

NSU

NTS

NZCOM

PGD

RANZCOG

RCTs

STDs

UK

USA

3DUS
General Practitioner

In Vitro Fertilisation

Second trimester maternal serum (blood) screening.

National Screening Unit

Nuchal Translucency Scanning

New Zealand College of Midwives

Pre-Implantation Genetic Diagnosis

Royal New Zealand and Australian College of Obstetricians and Gynaecologists

Random Controlled Trials

Sexually Transmitted Diseases

United Kingdom

United States of America

Three Dimensional Ultrasound 


\section{Appendix 3}

Information Sheet for Participants

\section{Study Title: "Making Sense of the Experience of Prenatal Screening"}

You are invited to take part in research examining the social impact of new genetic technologies, in particular, pregnant women's experiences of prenatal screening (including ultrasound scans to detect genetic abnormality, CVS (chorionic villus sampling) and amniocentesis).

\section{What is the purpose of the research?}

The research aims to learn more about the ways individual women experience and make sense of the choices they are confronted with if/when prenatal screening is offered, and, if they accept screening, when the possibility of abnormalities in their unborn baby is discovered. The study will also examine the experiences of those women who choose not to have screening during their pregnancy. The ways in which people make sense of the idea of "ethical decisions" relating to pregnancy within their own life experience, and more broadly within our society is a key theme of the research.

Approval for this research has been granted by the Central Regional Ethics Committee.

\section{What is the benefit of taking part in the research?}

A benefit for participants will be the sense of being able to contribute to increasing knowledge in society about the impact of these issues on the lives of individual people, and ensuring that the voices of the people who are affected by new technologies (in particular pregnant women) are heard and valued in society.

\section{Who can take part?}

While prenatal genetic screening is not routinely offered to all pregnant women in New Zealand, it is increasingly common for women in New Zealand over the age of 35 years to be offered screening due to being considered to be at a higher than average risk of carrying a baby with genetic abnormalities such as Down Syndrome. However, other women may have been offered or sought screening because of concerns such as the possibility of passing on familial disorders to their children, or a personal belief that screening is necessary in any case. Therefore, any woman who has considered having or had prenatal screening is welcome to participate in the research, as the study is about the experience of considering having screening, the weighing up the possible pros and cons, rather than the screening procedures themselves.

\section{What will my participation involve?}

Participants will take part in one interview, lasting approximately one hour. You will be asked questions about your own attitudes and experiences relating to prenatal screening. I realise that for many women this is in an extremely sensitive and personal topic and an issue which generates mixed feelings and complex responses. For this reason participants are welcome to bring a support person to the interview if they feel this necessary. In addition, participants are free to stop at any point during an interview for a break, or may choose to withdraw from participation in interviews altogether if they feel unable to continue. Your participation in this research will be confidential: you will not be identified in any report or publication that results from the research. All interview notes and tape recordings will be stored confidentially and securely and will be accessible only to myself and my research supervisor who is required to ensure your participation within the research remains 
rview notes and audio tape recordings will be stored anonymously and ars after the research has been completed. Participants may see and edit a terview transcript and at the conclusion of the study will have access completed research.

that the interviews will take place from mid-October to Mid-

\section{archer}

is Sarah Donovan and I am currently undertaking a $\mathrm{PhD}$ in Sociology versity. I am also a registered NZ midwife (not currently practising), ience in confidentially handling personal information, as well as ide women experiencing sensitive and highly personal issues related to birth, including termination of pregnancy.

like to take part in an interview for this research, or have about the idea of participating, please contact me: asara@student.vuw.ac.nz Tel: 044636710. c/- School of Social and Cultural Studies, Victoria O Box 600, Wellington. anonymous. Int destroyed two y copy of their in to a copy of the It is envisaged November 200

About the res Hello my name at Victoria Uni and have exper working along pregnancy and

If you would any question email: donor

S. Donovan University, H 


\section{Appendix 4}

Interview Questions

What is your personal experience of prenatal screening?

\{I.e. if you have undergone prenatal screening, what tests did you have? What were the results of the test/tests and what was your action at this point

While you were considering the options screening presented you with to have screening or not, which test you would have, what you would do depending on the outcome of tests etc... how did you feel?

How would you describe your own process of arriving at a decision that felt like the right one for you?

Did you ever feel that you "should" undergo screening, even if you personally didn't want to/ feel the need to have it? Why do you think you had this feeling?

Do you wish anything could have been different in your experiences around prenatal screening?

Did your own experience ever lead you to wish that technologies such as prenatal screening were not available?

In light of your own experience, what thoughts (if any) might you have for a friend who has become pregnant?

Do you feel comfortable with the possibility that as medical technologies develop, screening for more conditions is likely to be offered to pregnant women?

What does the idea of "ethics" mean to you?

Do you think prenatal screening should be a matter for public debate? Do you have any thoughts about the relationship between individual choice about prenatal screening and attitudes in society about prenatal screening?

Are there any other conditions for which you would like to see prenatal screening made available to pregnant women?

Are there any conditions which may occur in unborn babies which you feel should not be screened for? 
Do you believe that medical technologies such as prenatal screening benefit society? In what way?

Do you believe screening should be offered to all pregnant women, or only some? Who should be offered screening during pregnancy and why?

Who, if anyone, do you feel should be responsible for placing limits on what conditions or abnormalities should be screened for in society?

At present in New Zealand, as overseas, decisions about the ethics of new forms of health care, including what kinds of prenatal screening should be available to the public, are usually made after a process of public consultation, new legislation and the involvement of ethics committees. Have you ever been aware of opportunities for the public to be involved in this process?

What is your opinion about public involvement in such decisions?

Do you feel comfortable with your own level of awareness about the process through which new methods of prenatal screening become approved and available to the public in New Zealand?

Why? Why not?

It is likely that new methods of prenatal screening for genetic problems will continue to be developed, and that new ethical questions will be raised as these technologies expand.

Having faced decisions relating to the use of prenatal screening technologies yourself, do you have any comments or suggestions about way in which the introduction of new screening procedures should be considered and regulated in New Zealand? 


\section{Appendix 5}

Feature article on the research

Dominion Post, September 28, 2005.

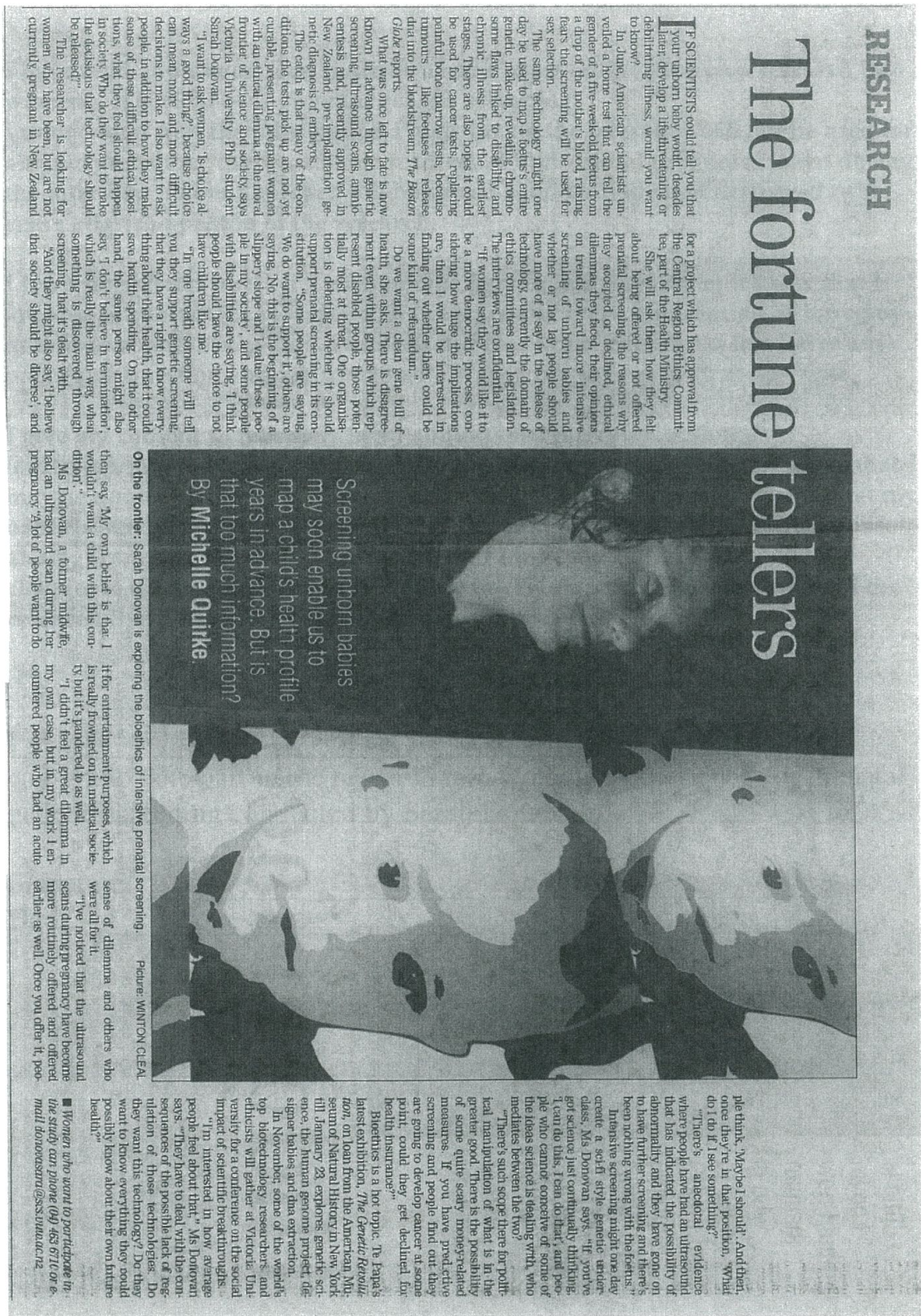




\section{Appendix 6}

Consent Form for Participants

I have read and understood the information sheet on the proposed research into women's experiences of prenatal genetic screening.

I understand that the information I give during the interview will not be used for any purpose other than academic research. I understand that notes taken during the interview and audio-tapes will be stored confidentially and destroyed two years after completion of the research.

I have considered whether I wish to take part in this research and know that I am able to contact the researcher if I require any more information or have any questions about taking part. I understand that I may choose not to answer any particular question and am free to withdraw from the research at any time.

I give my consent to take part in an audio-taped interview with the understanding that my participation in the research is confidential. I agree that I may be quoted in the written research as long as I remain confidential in that my name or any identifying information will not appear anywhere in publications arising from this research. I am aware that I may see and, if I wish, make changes to a copy of the transcript of my interview, and at the conclusion of the research that I have access to a final copy of research.

I [Full name] consent to take part in the research into women's experiences of prenatal genetic screening conducted by Sarah Donovan.

Signature:

Date: 


\section{Appendix 7}

List of approved indications for obstetric ultrasound, (U.S. Department of health and Human Services, 1984) ${ }^{212}$

- Estimation of gestational age for patients with uncertain clinical dates, or verification of dates for patients who are to undergo scheduled elective repeat caesarean delivery, indicated induction of labour, or other elective termination of pregnancy.

- Evaluation of fetal growth (e.g. when the patient has an identified etiology for utero-placental insufficiency, such as severe pre-eclampsia, chronic hypertension, chronic renal disease, severe diabetes mellitus, or for other medical complications of pregnancy where fetal malnutrition, i.e., IUGR or macrosomia, is suspected).

- Vaginal bleeding of undetermined etiology in pregnancy.

- Determination of fetal presentation when the presenting part cannot be adequately determined in labour or the fetal presentation is variable in late pregnancy.

- Suspected multiple gestation based upon detection of more than one fetal heartbeat pattern, or fundal height larger than expected for dates, and/or prior use of fertility drugs.

- Adjunct to amniocentesis.

- Significant uterine size/clinical dates discrepancy.

- Pelvic mass detected clinically.

- Suspected hydatidiform mole on the basis of clinical signs of hypertension, proteinuria, and/or the presence of ovarian cysts felt on pelvic examination or failure to detect fetal heart tones with a Doppler ultrasound device after 12 weeks.

- Adjunct to cervical cerclage placement.

- Suspected ectopic pregnancy or when pregnancy occurs after tuboplasty or prior ectopic gestation.

${ }^{212}$ Abridged, as cited in Taylor (1998). 


\section{Appendix 8}

Sample Nuchal Translucency Scan (NTS)

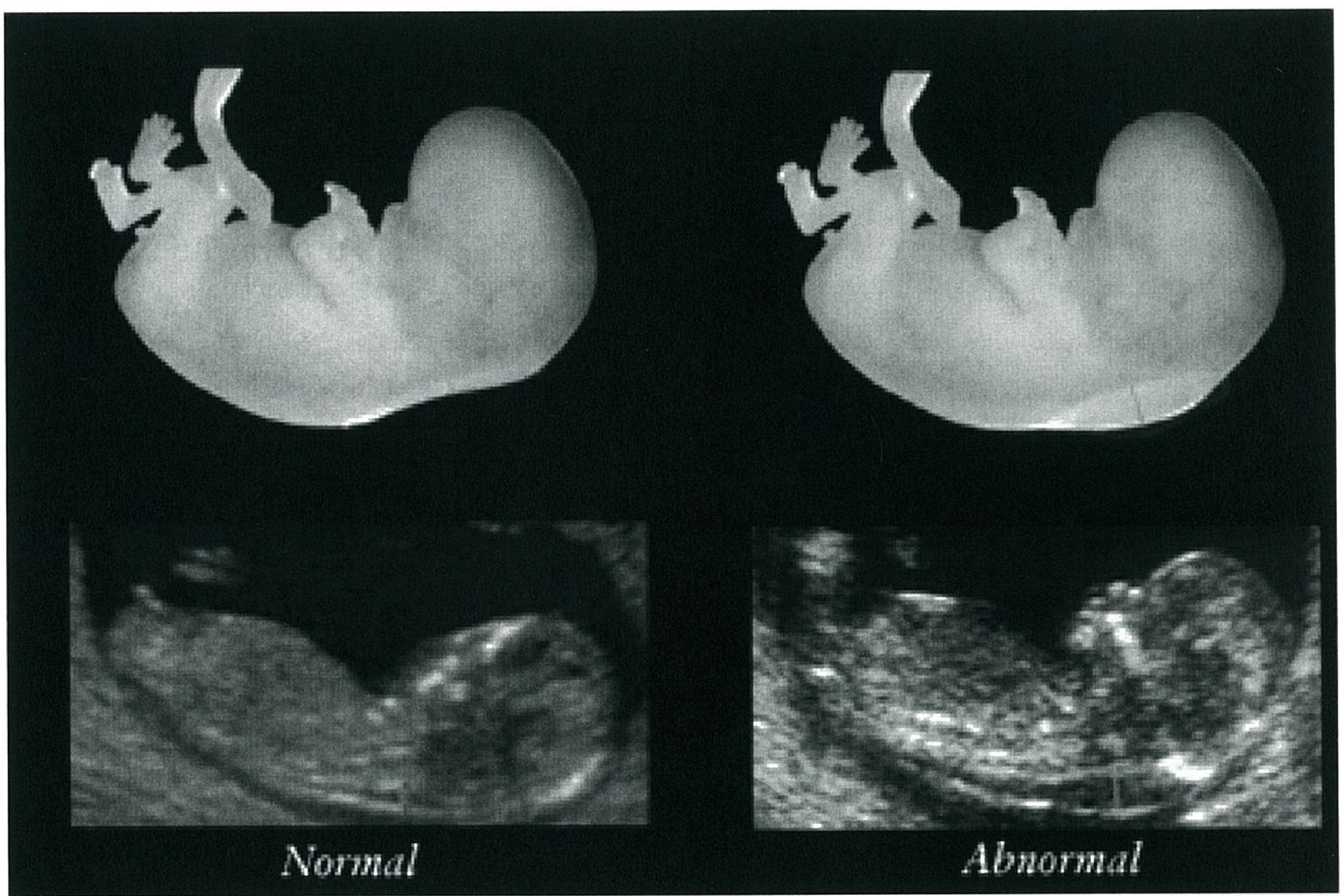




\section{Appendix 9}

Statement on ultrasound, New Zealand College of Midwives

\section{NZCOM CONSENSUS STATEMENT \\ Ultrasound}

This Consensus Statement was ratified at AGM 25 August 1995

The New Zealand College of Midwives does not recommend the routine use of ultrasound, including doppler and cardiotocographic monitoring, during pregnancy and childbirth.

The NZCOM recognises that the selective use of ultrasound, with the woman's informed consent, is appropriate where there are specific individual indications.

\section{References}

Title: $\quad$ Ultasound screening in pregnancy: a systematic review of the clinical effectiveness, cost effectiveness and women's views

Author: $\quad$ Bricker, L, Garcia, J, Henderson, J, Mugford, M, Neilson, J, Roberts, T, Martin, M-A.

Source: $\quad$ Health Technology Assessment 2000; Vol 4: No 16 http://www.ncchta.org/execsumm/summ416.htm

Title: $\quad$ Indications for ultrasound scanning

Author: $\quad$ Ultrasound utilisation working group for Ministry of Health

Source: $\quad$ Section 88, Maternity Services Notice, Ministry of Health, July 2002.

Title: $\quad$ Ultrasound for foetal assessment in early pregnancy

Author: Citation: Neilson J P

Source: Cochrane Review: The Cochrane Library, Issue 2, 2002 http://www.medscape.com

Title: $\quad$ A Guide to Effective Care in Pregnancy and Childbirth, Chapter \& Oxford University Press, 2000

Author: $\quad$ Enkin, M Keirse, Marc JNC Chalmers, I

Title: $\quad$ Routine ultrasound in late pregnancy (after 24 weeks gestation)

Author: $\quad$ Bricker L, Neilson J

Title: $\quad$ Pursuing the Birth Machine

\section{Author: Wagner, $\mathrm{M}$.}

Source: $\quad$ Ace Graphics. Australia, 1994.

ISBN 0-64-616837-1

Title: $\quad$ Ultrasound scans during pregnancy (brochure)

Source: $\quad$ Women's Health Action Trust, PO Box 9947, Newmarket, Auckland Phone (09) 520-5295 Fax (09) 520-4152 - 80 cents each guidelines are designed to educate and support best practice. 


\title{
Appendix 10
}

\section{nzherald.co.nz}

\section{Rise in Down syndrome babies blamed on lack of screening}

\author{
12:00A M Saturday September 17, 2005
}

More New Zealand babies are being born with Down syndrome, despite improvements in prenatal testing technology.

Numbers rose from 50 in 1996 to 80 in 2002, and experts blame the lack of a co-ordinated nationa! screening programme.

"We don't have a screening programme in NZ and what we are doing at the moment is ad hoc, said Professor Peter Stone, head of the University of Auckland's department of obstetrics and gynaecology.

"We haven't really embraced it as a health issue for the population at risk, which is all pregnant women."

Professor Stone was commissioned by the Ministry of Heath's National Screening Unit to determine how antenatal screening for Down syndrome should be done, following a workshop in April 2004.

The workshop tound antenatal screening "was not effective or sustainable" and had developed haphazardly with no co-ordination, no audit and no quality control.

"It appears that the vast majority of women are not being offered screening." the workshop reported.

Ministry statistics confirm the claims. In 1996, the number of Down's babies per 1000 births was 0.87 . By 2002, the incidence had risen to 1.48. The natural incidence of Down syndrome - if there are no terminations - is 1.67 per 1000 births.

Professor Stone, whose report is due at the end of the year, is unable to shed any light on why screening is such a shambles.

He pointed out that one ultrasound test, done from 10 weeks into pregnancy, was partially Covernment funded. "l don't know how it is that a test can be introduced with funding, but not be introduced as part of a co-ordinated approach."

Another maternal serum :est was originally funded, but now costs $\$ 75$, meaning few mothers have it done.

Dr Pat Tuohy, chief adviser on child and youth health, said the ministry was reviewing the funding of maternity care which was an opportunity to address the quality and safety of ultrasound scanning and other issues relating to screening.

But if a more co-ordinated programme is introduced that has the effect of reducing the incidence of 
Down's, it might conflict with the views of some disability groups concerned about efforts to remove imperfection. The Down Syndrome Association's position statement on prenatal testing says people with Down syndrome have a right to life.

It advocates that testing be used to prepare parents for birth, that up-to-date information should be available to the professionals involved, and that people should not be pressured into having testing.

This story was found at:

Copyright Q2007, APN Holdings NZ Limited 


\section{Appendix 11}

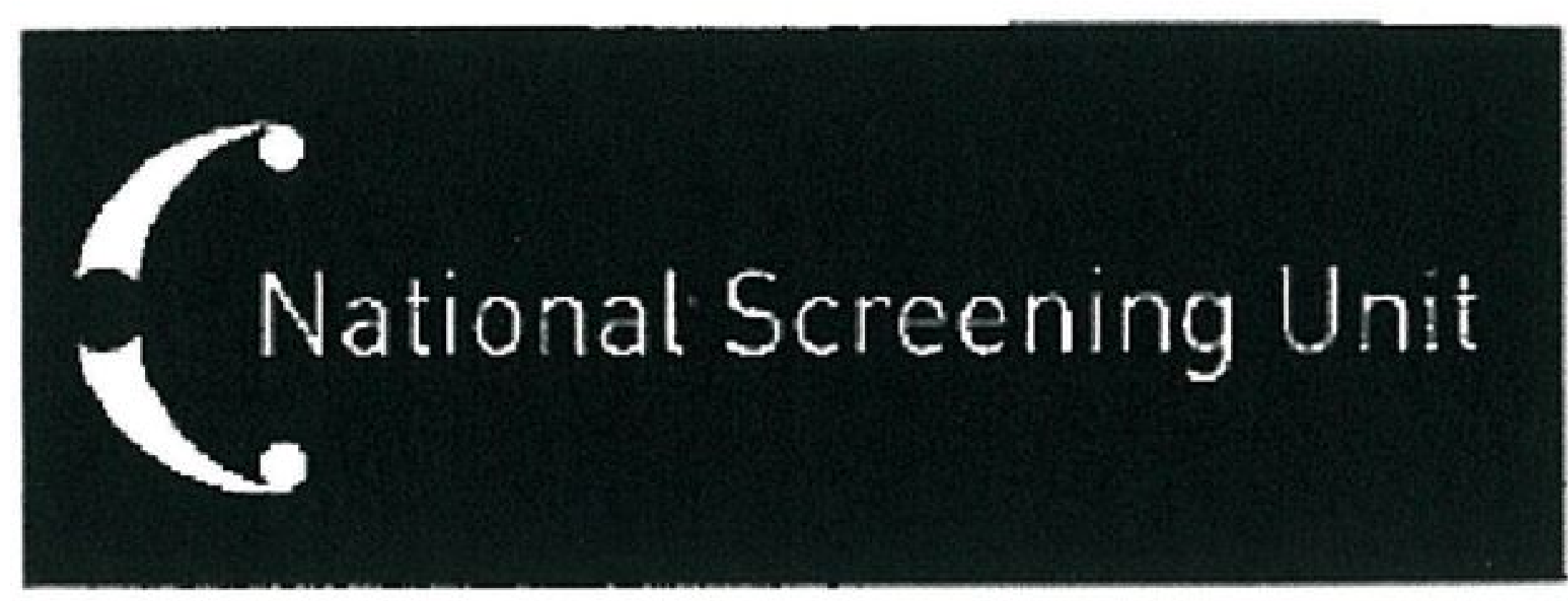

You are here: bome news media releases

\section{Media Releases}

Thursday, 26 April 2007

\section{Bepgrt on Antenatal Down the Nationalscenng Unit by}

Antenatal Down Syndrome Screening in New Zealand 2007 is the result of work carried out by the Antenatal Down Syndrome Screening Advisory Group, which was convened as part of a review of the current practice of antenatal screening for Down syndrome undertaken by the National Screening Unit.

The Advisory Group unanimously agreed that the current practice of screening using only maternal age and/or nuchal translucency (NT), without biochemical markers is unsafe, inequitable, and should not continue. The Advisory Group made a number of recommendations for ways to improve the safety and quality for antenatal Down syndrome screening.

Advisory Group Chair Dr Paul Harper says current screening methods commonly used are not as reliable and accurate as other methods that are available. This results in too many women having diagnostic tests, and as these have a small risk of miscarriage (about 1 to 2 percent), pregnancies are being lost unnecessarily.

National Screening Unit Manager Karen Mitchell says the Advisory Group had a difficult task, given the wide range of views represented and the need to consider the technical, social and ethical issues of antenatal Down syndrome screening. The Minister of Health, Hon Pete Hodgson, has requested further work to be carried out to look at how a nationally organised screening programme for antenatal Down syndrome could be implemented.

Mitchell said that the primary intention of a national screening programme would be to improve the quality and safety of screening practice.

"The key driver is to improve the safety and quality of screening practice for women in New Zealand. The work of the Advisory Group provides a clear way forward to do this," she says.

"A national screening programme would include an informed consent process, national standards, monitoring and evaluation. The National Screening Unit will continue to work 
with key stakeholders including colleges, health care providers and the New Zealand Down Syndrome Association as it carries out this work."

Mitchell said the further work requested by the Minister would be completed by October 2007.

Download a copy of 'Antenatal Down Syndrome Screening in New Zealand 2007

For further information contact:

Michael Flyger

Media Advisor

Ministry of Health

Ph: 044962265

Mobile: 0274346878

\section{Questions and Answers}

\section{Why was the Advisory Group establised?}

International research indicates that safer and more reliable tests could be used in New Zealand to screen for Down syndrome. The tests currently used can result in women being unnecessarily referred for an invasive diagnostic procedure, for which there is a small risk of miscarriage ( 1 to 2 percent).

There are better methods of screening that can be implemented, and these should be accompanied by high quality information, a robust informed consent process, audit, monitoring, and evaluation.

\section{What did the Advisory Group do?}

This Advisory Group considered the technical, medical, social and ethical issues relating to screening. The Advisory Group developed its advice over an intensive six-month period and completed its report to the National Screening Unit in January 2007.

\section{Who was on the Antenatal Down Syndrome Advisory Group?}

Members of the Advisory Group were appointed for their expertise and experience. It included:

\begin{tabular}{|c|c|}
\hline Name & Expertise \\
\hline Faul Karper (Cheir) & Down syndrome and a disabiley perspecive \\
\hline Norma Campoea & Miewitery \\
\hline Sharron cole & Wormen's health \\
\hline Fea Daelinbach & Women's health \\
\hline Clive felix & Cytogenetics \\
\hline John Forman & Rare disorders anc a disablity perspective \\
\hline Owon Hughes & Government policy relating to disability \\
\hline Nikki Kerruish & Paediatrics and health ethics \\
\hline
\end{tabular}




\begin{tabular}{|c|c|}
\hline Pippe Kyle & Obstetrics and fetal medicine \\
\hline Fiona Mocrimmon & Heath law \\
\hline Racheal NcEwing & Rachology/sconography \\
\hline Katherine Neas & Clinical genesics \\
\hline Anne o'Connar & Down syndrome and a disability perspective \\
\hline Peter Stone & Obstetrics and fetal medicine \\
\hline Mere Wallace & Soclat work and a M sori health perspective \\
\hline Dianne Webster & Biochemical genetiss \\
\hline Wend: Witks & Disability perspectives \\
\hline Jorathon Wilcox & General practice \\
\hline Lynda Wallams & Women's healk $\mathrm{h}$ \\
\hline
\end{tabular}

\section{What were the recommendations of the Advisory Group?}

The Advisory Group unanimously agreed that the current practice of screening using only maternal age and/or NT, without biochemical markers is unsafe, inequitable, and should not continue. However, there was not unanimous agreement on the best way of addressing concerns about current practice. The Advisory Group made 29 key recommendations.

\section{What else has been done to look into this issue?}

The National Screening Unit contracted Professor Peter Stone (Head of Department of Obstetrics and Gynaecology, University of Auckland) to undertake a project to assess the current practice of antenatal screening for Down syndrome in New Zealand, and advise on future delivery of antenatal screening for Down syndrome. Professor Stone delivered his report in early February 2006. Amongst other things, Professor Stone recommended "that the current situation of age and Nuchal Translucency screening leading to invasive testing be urgently reviewed with the view to stopping this as a screening practice".

The National Screening Unit also commissioned a systematic review of the literature. This review is available at:

http://nzhta.chmeds.ac.nz/publications/downs.pdf

\section{What have the Ministry and the Minister decided?}

The Minister of Health has considered the report of the Advisory Group and has asked for more detailed work to be carried out to determine how a nationally organised programme for antenatal Down syndrome could be implemented in New Zealand.

\section{What steps have already been taken to improve current screening practice?}

In 2006, the Ministry of Health wrote to relevant health professionals and services, advising them of the concerns and providing advice on the safest forms of screening available in New Zealand.

Requirements have also been placed on practitioners performing Nuchal Translucency ultrasounds scans. From 1 July 2007, all health professionals performing these scans under the Section 88 Maternity Services Notice must have the appropriate training and 
use appropriate equipment. They will be required to be have obtained the appropriate accreditation recognised by the Royal Australian and New Zealand College of Obstetricians and Gynaecologists and the Royal Australian and New Zealand College of Radiologists.

\section{Will a national screening programme be introduced?}

This is a complex area. Before a decision is made, a great deal of detailed work needs to be carried out to look at how a programme could be implemented.

\section{When will a decision be made about whether a national antenatal Down syndrome screening programme will be introduced?}

Advice on how a programme could be implemented will be provided to the Minister of Health by 30 October 2007 .

\section{What is a national screening programme?}

A national screening programme 'organises' all element of the screening and diagnostic pathway. At a national level it includes planning, co-ordination, monitoring and evaluation of all activities along the screening pathway to make sure the services are safe and of high quality.

\section{What would be the advantages of a national screening programme?}

Implementing a national screening programme would help to ensure that women who choose to have screening are offered the safest and most reliable form of screening possible. It would also help to ensure that invasive diagnostic procedures are limited to women who have an increased chance of having a child with Down syndrome and who have made an informed choice to use those tests.

Implementing a 'best practice' form of screening is complex and involves co-ordinating the activities of a range of health practitioners over two trimesters of pregnancy. It needs to be implemented in a co-ordinated and nationally consistent manner, with robust quality assurance processes. The structures of a national screening programme could help the sector to implement 'best practice'.

\section{Would a national programme mean that screening for Down syndrome is compulsory?}

No. All national screening programmes are based on consumers giving informed consent to the screening and diagnostic pathway. Women would be able to decline screening, decline diagnostic tests, and make their own decisions about their pregnancy. Women would need to be offered access to appropriate counselling and support services.

\section{What is Down syndrome?}

Down syndrome is a life-long condition that causes delays in learning and development. It is caused by the presence of an extra copy of chromosome 21 (i.e., three instead of two). This is also called trisomy 21. Down syndrome occurs in approximately one in seven hundred pregnancies, and about șeventy children are born with Down syndrome each year in New Zealand. For more information about Down Syndrome see the New Zealand Down Syndrome Association website. 


\section{What is the purpose of antenatal screening for Down syndrome?}

The purpose of antenatal screening for Down syndrome is to provide pregnant women, who choose to have the screening tests, with information about whether the fetus is likely to have Down syndrome or some other fetal anomaly. This information may help women to make informed decisions and plans about their pregnancy, including:

- whether to have diagnostic testing

- whether to continue with or terminate the pregnancy

- preparing for the birth and life of a child with Down syndrome or other condition

- planning to give birth at a specialist centre that can provide any immediate neonatal treatment, if necessary.

\section{What is a screening test?}

A screening test is offered to people who do not have any symptoms or signs that they have a particular condition. It predicts the likelihood of a person having or developing a particular condition. With antenatal screening for Down syndrome, screening tests can identify pregnant women whose fetus have an increased risk of Down syndrome and who might benefit from further investigation. These women are offered a diagnostic test (discussed further below).

\section{Are screening tests 100 percent accurate?}

Screening tests cannot tell you whether the fetus does have Down syndrome. Screening tests sort women into two groups: women who are low-risk and do not have an increased chance that their fetus has Down syndrome, and women who are high-risk and do have an increased chance that their fetus has Down syndrome. There will be some falsenegative results and some false-positive results.

\section{What is a diagnostic test?}

A diagnostic test states if the condition definitely is present or not. Diagnostic tests are often expensive and carry risks. The use of these tests should be limited to wamen who have an increased risk of having the condition.

\section{What screening tests for Down syndrome are currently used in New Zealand?}

- Advanced maternal age: women aged over 35 years are offered a diagnostic test. Screening on the basis of maternal age results in significant numbers of women being referred for diagnostic tests, which carry a risk of fetal loss. Maternal age screening means that women who are less than 35 years of age are advised they have a low risk, and are not offered other forms of screening or diagnostic tests. Although older women are more likely to have a child with Down syndrome, more children with Down syndrome are born to younger women due to the fact that there are more births in this age bracket.

- Nuchal translucency (NT) ultrasound scan: currently all women consulting a health practitioner in their first trimester of pregnancy can be offered a nuchal translucency ultrasound scan, which receives public funding. The screening test involves measuring the depth of fluid at the nape of the fetal neck. The test is performed during an early ultrasound scan (11-14 weeks of pregnancy). An increased amount of fluid may indicate that the fetus has Down syndrome or another chromosomal, structural or genetic anomaly. NT scans should only be performed by highly skilled 
professionals. From 1 July 2007, practitioners performing NT scans under the Section 88 Maternity Services Notice will need to be accredited by a recognised organisation.

- Maternal serum screening: Women who do not consult a health practitioner in their first trimester of pregnancy can have a second trimester maternal serum screening test. This test is not publicly funded. It costs approximately $\$ 75$ and is available from LabPlus (Auckland DHB). It involves taking blood from the woman at 14-20 weeks of pregnancy and measuring the concentration of various biochemical markers in the blood.

\section{What is the age-related risk of having a child with Down syndrome?}

Levels of risk of having a Down syndrome pregnancy in relation to a woman's age

\begin{tabular}{|l||l|l|}
\hline Woman's age & Risk as a ratio & $\%$ risk \\
\hline 20 & $1: 1500$ & 0.065 \\
\hline 30 & $1: 800$ & 0.125 \\
\hline 35 & $1: 270$ & 0.37 \\
\hline \hline 40 & $1: 100$ & 1.0 \\
\hline \hline 45 znd ever & $1: 150$ and greater & 2.0 \\
\hline
\end{tabular}

Why are the newer methods of screening for Down syndrome better than using maternal age?

If 50,000 women were screened on the basis of maternal age, approximately 20 percent of them (i.e. 10,000 women) would be considered high-risk and would be offered a diagnostic test. The diagnostic test has a 1 percent risk of miscarriage. If we screen 50,000 women on the basis of their maternal age, we could expect this to result in 100 miscarriages.

However, if the most reliable form of screening is used to screen 50,000 women, the 20 percent of women offered diagnostic tests could be reduced to around 1 percent (i.e. 600 women). Instead of 100 miscarriages we would expect to have about 6 miscarriages.

\section{What diagnostic tests for Down syndrome are currently used in New Zealand?}

Approximately 3,500 diagnostic tests are currently performed each year in New Zealand. These procedures carry a risk of miscarriage (1-2 percent).

Amniocentesis: an invasive procedure whereby a sample of amniotic fluid is removed and sent for analysis.

Chorionic villus sampling (CVS): an invasive procedure where placental tissue is removed and sent for analysis. 


\title{
Appendix 12
}

\author{
NZ Ministry of Health Web Page \\ Printed 17/11/2009 04:11:10 p.m. Back Print
}

\section{Media Release}

8 June 2006

\section{Down Syndrome Antenatal Screening Under Review}

\author{
A large number of health professionals are being advised of concerns relating to the current practice of \\ antenatal screening for Down syndrome.
}

Manager of the National Screening Unit, Karen Mitchell, says letters have been sent to heaith professionals highlighting current concerns, which include referrals for diagnostic procedures (amniocentesis and chorionic villus sampling) and of steps they can take to improve current practice and ensure that women are better informed.

The National Screening Unit commissioned Professor Peter Stone from the University of Auckland to assess current practice

of antenatal screening for Down syndrome in New Zealand. Professor Stone's Report states that current practice is "increasingly expensive and labour intensive". It also suggests that "more normal pregnancies may be adversely affected by amniocentesis than the numbers of Down syndrome fetuses detected." Amongst other things, the report recommencs that current practice be urgently reviewed.

The National Screening Unit is to review current practice and with the help of an External Advisory Group will provide initial advice to Minister of Health, Pete Hodgson, early next year.

Karen Mitchell says, "amniocentesis and chotionic vilius sampling, carry a $0.5 \%$ to $1 \%$ chance of miscarriage and should only be carried out where a woman is informed of the risks associated with the procedure and has given informed consent to the procedure."

"Ensuring that pregnant mothers have a safe pregnancy is our main goal. There are immediate steps that can be taken now to limit the risks associated with testing, and to ensure that women are better informed", says Karen Mitchell.

The letter states that women should not be referred for amniocentesis or chorionic villus sampling based on maternal age alone. All women consulting a health practitioner in the first trimester of pregnancy can be affered a Nuchal Translucency ultrasound scan, which is publicly funded. Women should also be informed about the difference between a screening test and a diagnostic test, and the risks associated with the different types of screening tests and diagnostic tests.

Women should also be informed as to whether the radiographer is accredited to perform a Nuchal Translucency ultrasound scan, which should be performed between 11 and 13 weeks and 6 days of pregnancy. These scans shouid only be performed by practitioners who have current accreditation.

\section{Questions and Answers}

1. What are the concerns with amniocentesis and chorionic villus sampling (CVS)?

Amniocentesis and chorionic villus sampling procedures carries a small risk of miscarriage $\{0.5-1$ percent). Concerns have been raised that these tests are being provided to women who may not have an increased chance of having a child with Down syndrome and who have not been informed about the risk of pregnancy loss associate with the tests. The use of these invasive diagnostic tests should be limited to women who have an increased chance of having a child with Down syndrome and who have made an informed choice to use those tests.

2 How will the Ministry limit the use of invasive diagnostic tests? 
The Ministry has written to all midwives, general practitioners, obstetricians, laboratories, tadiologists and radiographers to advise them of ways that current practice can be improved now. The use of invasive diagnostic tests can be reduced by offering each pregnant woman a screening test that assess her individual chance of having a child with Down syndrome. Each woman should also be informed of the risks associated with the screening tests and the diagnostic tests. This will allow women to make informed decisions about the use of amniocentesis and chorionic villus sampling.

\section{What antenatal Down syndrome screening is currently available?}

Currently all women consulting a health practitioner in their first trimester of pregnancy can be offered a Nuchal Translucency ultrasound scan, which is publicly funded. Women who do not consult a health practitioner in their first trimester of pregnancy can have a second trimester maternal serum screening test. This test is not publicly funded at present; it costs approximately $\$ 75$ and is avalable from Lab Plus (Auckland $\mathrm{DHB})$.

\section{Who will make up the external advisory group?}

Members appointed include consumers with expertise in women's health and maternity services, representatives of the disability sector, and others with experience in:

- ethics

- midwifery

- general practice

- obstetrics

- paediatrics

- cytogenetics

- clinical genetics.

The Advisary Group will have its first meeting in June 2006. The Chair of the Group is still to be decided.

5. Does the Ministry aim to reduce the number of babies born with Down syndrome every year? No, this is not about reducing the numbers of babies born with Down syndrome. This is about ensuring that screening services offered to women during pregnancy do not cause unnecessary harm to women and their pregnancies.

\section{ENDS}

Related information

Down Syndrome Screening

\section{Contact Details}

For interviews with Karen Mitchell, Ministry of Health's National Screening Unit Manager, please phone media advisor Victoria Evans

- 104) 4962036 or 021802622

For interviews with Zandra Vaccarino, National Coordinator New Zealand Down Syndrome Association, please phone - 0800693724 then dial 1 


\title{
Appendix 13
}

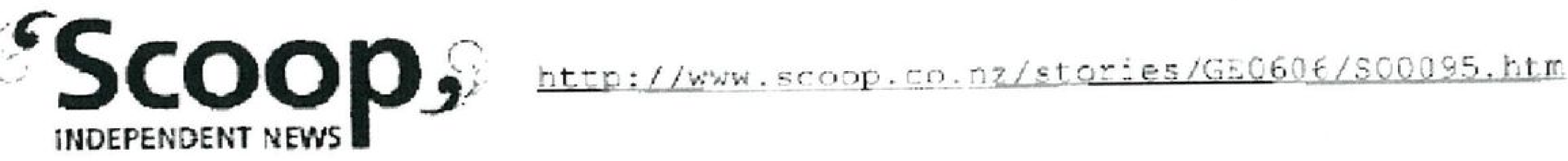

\section{DPA disputes antenatal screening claims}

\author{
Tuesday, 20 June 2006, 12:01 pm \\ Press Release: Disabled Persons Assembly
}

Media Release

For Immediate Release

20 June 2006

DPA disputes antenatal screening claims

DPA, a national assembly for disabled people, is concerned that the full report into antenatal screening for Down syndrome is at odds with National Screening Unit claims about the issue.

The report by Peler Stone clearly states that evidence shows the current screening process "has failed to reduce the number of babies born with undiagnosed Trisomy 21 [Down syndrome]".

"This is in direct contrast to the National Screening Unit claims that the review is not about reducing the number of babies bom with Down syndrome," says Mike Gourley, President of DPA.

"While DPA agrees that current screening processes need to be reviewed, we are alarmed that this may be happening with a view to eliminating a group of disabled people.

"Currently, the report and surrounding discussions are focused solely on the number of "healthy" foetuses that may suffer through current screening practices. The rights of people with Down syndrome are ignored which is of great concern," he says.

Mr Gourley says that people born with Down syndrome are not a cost to society and their skills. talent and potential need to be respected.

"The nature of the screening programme means it is very difficult to have representatives with Down syndrome on the review committee. Who'd want to sit listening to others talk about eradicating them? Nevertheless, a committee discussing a process that directly affects people with Down syndrome must find ways to include the people who live with the syndrome," he says.

ENDS 


\title{
Appendix 14
}

\section{Background to the quality improvements}

\author{
home $>$ for health professionals $>$ antenatal screening for down syndrome - quality
}

improvement measures $>$ background to the quality improvements

\section{Background to the quality improvements}

A report released in April 2007 by the National Screening Unit, Antenatal Down Syndrome Screening in New Zealand 2007, is the result of work carried out by the Antenatal Down Syndrome Screening Advisory Group, which was convened as part of a review of the current practice of antenatal screening for Down syndrome undertaken by the National Screening Unit.

The Advisory Group unanimously agreed that the current practice of screening using only maternal age and/or nuchal translucency (NT), without biochemical markers is unsafe, inequitable, and should not continue. It said current screening methods commonly used are not as reliable and accurate as other methods that are available. This results in too many women having diagnostic tests, and as these have a small risk of miscarriage (about 1 to 2 percent). The Advisory Group made a number of recommendations for ways to improve the safety and quality for antenatal Down syndrome screening.

Following the stakeholder workshops in mid 2007 the Ministry of Health has undertaken detailed implementation planning to ensure the quality improvement of current screening. In late 2007 the government agreed to fund improvements to the quality of screening for Down syndrome. The purpose of these quality improvements is to ensure that women who choose to have screening will be able to access the safest and most reliable form of screening available. Women who choose not to be screened will be supported in their decision.

The initial measure will be the funding of the second trimester maternal serum test from August 2008. This is to improve the access to screening and is primarily for women who have not had a nuchal translucency (NT) scan or who choose to wait for a combined result with their NT scan and arrange this with their ultrasound practitioner.

Other quality improvements which will be implemented by mid 2009 will include the development of:

- a range of screening tests/pathway options that can be accessed by women who present in the first and/or second trimester

- information for pregnant women and their partners

- information and standards for practitioners on appropriate screening tests and protocols

- standards for ultrasound services and laboratories

- improved access to counselling, support services and genetic services

- monitoring of laboratories, ultrasound services and pregnancy care providers. 
The National Screening Unit will plan, coordinate and monitor these quality improvements with expert advice from key groups and organisations from the health and disability sector. Please feel welcome to contact Nicola Deveraux, Programme Manager Antenatal

Screening, on (09) 5809243 or email nicola_deveraux@moh.govt.nz if you would like to discuss this in more detail. 


\section{Appendix 15}

Back to previous page I Go to the health section

\section{Screening on the way for Down syndrome babies}

Published: 4:03PM Sunday November 08, 2009

Source: NZPA

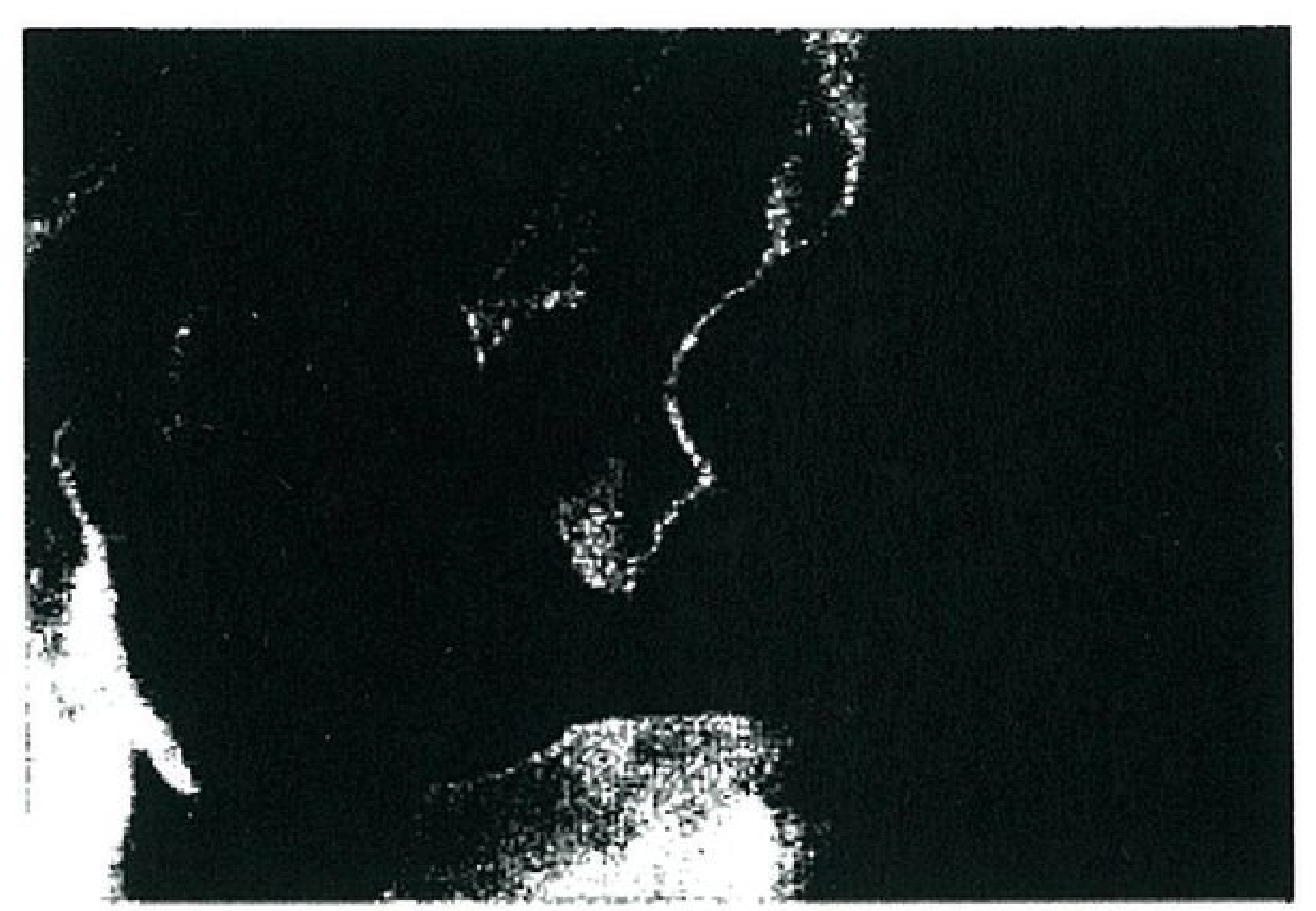

Source:

New Zealand may get a national screening programme for Down syndrome birth defects next year - four years after health officials were told some of the present testing is not safe enough.

But political sensitivities continue to dog the project and officials have suggested calling it a "quality improvement" rather than a national screening programme, according to a senior academic.

The Health Ministry's antenatal screening unit called in 2007 for changes to improve antenatal screening for Down syndrome.

As well as sensitivities around abortion and eugenics - aborting foetuses with disabilities before birth - a cultural issue has to be overcome.

The proposed effective screening method is to combine blood tests with an ultrasound exam - a "nuchal translucency (NT) test" to measure the thickness of the back of the neck of the foetus - in the first 13 weeks of pregnancy, the first trimester.

"Unfortunately a large number of women in New Zealand still present to a health carer for the first time later than this," Peter Stone, professor of maternal foetal medicine at Auckland University, told NZPA.

"This is a socio-cultural issue." 
That was a major reason why doctors continued to offer second trimester serum testing.

The NT testing is reported to be more than 80 percent accurate, with a low risk of falsely indicating Down syndrome in a healthy foetus, and women can be given numerical odds of their carrying an affected baby.

A woman determined to be at high risk by the NT test then still has time for an invasive test to tell for sure.

The ministry's advisory group which canvassed a national screening programme in 2007 reached a consensus that New Zealand's approach to screening was unsafe. Stone had already told the unit in February 2006 that the current system should stop.

He suggested that "more normal pregnancies may be adversely affected by amniocentesis than the numbers of Down syndrome foetuses detected".

At least 3500 diagnostic tests with a risk of miscarriage are performed each year, including amniocentesis - removal of a sample of amniotic fluid for analysis - and removal of placental tissue for analysis.

The ministry unit previously noted the screening methods being commonly used result in too many women having diagnostic tests, with a risk of about 1 percent to 2 percent of pregnancies being lost unnecessarily.

Stone told NZPA he was still working with the unit trying to sort out a safer and more reliable form of screening but progress had been slow.

"There has been little wide debate because it has been difficult to engage those most affected... the currently or soon-to-be pregnant mothers and, of course, their partners."

Consultation and advice sought by the screening unit and "various changes which appear to be ongoing in the Ministry of Health and in the national screening unit have led to delays".

A ministry spokeswoman said public funding had been provided for second trimester blood tests last year, and from February 2010 funding would be provided for first trimester combined screening - the combined result from an NT scan and maternal blood tests.

Stone said the eventual screening could provide a platform to assess the "health" of the pregnancy and the baby early on, by including not only Down syndrome but other conditions that could place the woman at higher risk of pre-eclampsia or foetal growth restriction.

But, so far, the only change in the past two years had been the funding for second trimester blood tests - which was reinstating access that had previously been removed. 
Next year he hoped to see the funding and introduction of first trimester blood tests combined with the NT scan to give a combined risk estimate.

"At that point, NT alone will no longer be recommended to be done in isolation from first trimester blood testing," Stone said.

"There will in effect be national screening but due to complex sensitive political factors the advice from the national screening unit and the Director General of Health to the past and current Ministers of Health was not to call this a national screening programme but 'quality improvement'."

Copyright (C) 2009, Television New Zealand Limited 


\section{Appendix 16}

\section{Our Terms Of Reference}

Terms of Reference in Māori.

\section{Goal}

To enhance New Zealand's understanding of the cultural, ethical and spiritual aspects of biotechnology and ensure that the use of biotechnology has regard for the values held by New Zealanders.

\section{Role}

Toi Te Taiao: The Bioethics Council is a ministerial advisory committee to:

1. Provide independent advice to Government on biotechnological issues involving significant cultural, ethical and spiritual dimensions.

2. Promote and participate in public dialogue on cultural, ethical and spiritual aspects of biotechnology, and enable public participation in the Council's activities.

3. Provide information on the cultural, ethical and spiritual aspects of biotechnology.

\section{Key tasks}

In undertaking its functions the committee is expected to:

1. Advise the Government on biotechnological issues of national importance involving significant cultural, ethical and spiritual dimensions and guide decision-makers on these matters. The advice will be made public and should:

- Take account of the values of New Zealanders.

- Take into account the social context and dimensions of biotechnology and the issues it generates.

- Articulate and report on the diversity of views on an issue.

- Be guided by values and views held in common as well as identifying areas where a diversity of values and views remain, in particular those created by Treaty issues.

2. Demonstrate its commitment to the Treaty of Waitangi including its responsibility to consult and engage with Māori in a manner that specifically provides for their needs.

3. Act as a public forum for dialogue on ethical, cultural and spiritual dimensions associated with the use of biotechnology, and publish reports.

4. Develop and implement other strategies to involve, communicate and consult with the public and other stakeholders about the ethical, cultural and spiritual dimensions of biotechnology. These strategies will seek to contribute to the building of trust between the public and various stakeholders. 
to policy development in ethics and values with a view to promoting consistency and minimising duplication between existing ethics advisory bodies.

6. Maintain a watching brief, with a view to facilitating public understanding of underlying values and ethics, on:

- Different "world_views" and other interdisciplinary topics related to ö́technology.

- The implications of those world views to Treaty issues.

- Through a "future watch" function to monitor emerging issues and developments in biotechnology:

7. Establish and sustain links with similar international fora dealing with similar issues.

8. Develop the means to assess the effectiveness of its activities. 


\section{Bibliography}

Albers, L. (2001). Monitoring the fetus in labour: evidence to support the methods. Journal of Midwifery \& Women's Health, 46(6):366-373.

Alderson, P. (2001). Prenatal screening, ethics and Down's syndrome: a literature review. Nursing Ethics, 8(4):360-374.

Alfirevic, Z., Devane, D., Gyte, G.M.. (2006). Continuous cardiotocography (CTG) as a form of electronic fetal monitoring (EFM) for fetal assessment during labour. Cochrane Database of Systematic Reviews 2006, issue 3.

American College of Obstetricians and Gynaecologists (2007). Screening for fetal abnormalities. Washington: ACOG Practice Bulletin 77.

American Institute of Ultrasound in Medicine (1991). AIUM bioeffects committee: safety considerations for diagnostic ultrasound. Rockville MD: AUIM.

American Institute of Ultrasound in Medicine: AIUM practice guidelines for the performance of an antepartum obstetric ultrasound examination. Journal of Ultrasound in Medicine, 22:116.

Anleu, S.R. (1997). Reproductive autonomy and reproductive technology: gender, deviance and infertility. In K. Peterson (ed.), Intersections: Women on Law, Medicine and Technology. Dartmouth: Aldershot, pp. 99-125.

Antsaklis, A. J. (1998). Debate about ultrasound screening policies. Fetal Diagnosis and Therapy, 13(4):209-215.

Aronsen, J. (1994). A pragmatic view of thematic analysis. Qualitative Report, 2(1). Unpaginated. Retrieved 3 October 2007 from the World Wide Web: http://www.nova.edu/ssss/QR/BackIssues/QR2-1/aronsen.html

Armstrong, D. (2006). Embodiment and ethics: constructing medicine's two bodies. Sociology of Health and Illness, 28(6):866-881.

Armstrong, E. M. (1998). Diagnosing moral disorder: The discovery and evolution of fetal alcohol syndrome. Social Science \& Medicine, 47(120):2025-2042.

Australian Medical Association (2005). 'Doctors best placed to provide medical care for pregnant women'. Retrieved 1 August 2008 from the World Wide Web: http://www.ama.com.au/node/2094

Baillie, C., Mason, G. \& Hewison, J. (1997). Scanning for pleasure. British Journal of Obstetrics \& Gynaecology, 104:1223-1224. 
Baillie, C. \& Hewison, J. (1999). Obtaining selective consent to scanning, rather than screening, is possible. British Medical Journal, 318(7186):805-6.

Baillie, C., Smith, J., Hewison, J. \& Mason, J. (2000). Ultrasound screening for chromosomal abnormality: women's reactions to false positive results. British Journal of Health Psychology 5(4):377-394.

Beauchamp, T. L. \& Childress, J. F. (1994). Principles of Biomedical Ethics (4th edition). Oxford: Oxford University Press.

Beauchamp, T. L. \& Childress, J. F. (2001). Principles of Biomedical Ethics (5th edition). Oxford: Oxford University Press.

Beazoglou, T., Heffley, D., Kyriopoulos, J, Vintzileos, A. \& Benn, P. (1998). Economic evaluation of prenatal screening for Downs syndrome in the USA. Prenatal Diagnosis, 18:1241-1252.

Beck-Gernsheim, E. (2002). Reinventing the Family. Cambridge: Polity.

Begley, C.M. (1996). Using triangulation in nursing research. Journal of Advanced Nursing, 24(1):122-128.

Bennett, V.R. \& Brown, L.K. (1996). Myles Textbook for Midwives (12 ${ }^{\text {th }}$ edition). Churchill Livingstone: Edinburgh.

Bensing, J. (2000). Bridging the gap. The separate worlds of evidence-based medicine and patient-centred medicine. Patient Education \& Counselling, 39(1):17-25.

Berne-Frommel, K., Josefson, G. \& Kjessler, B. (1984). Who declines from antenatal serum alpha-fetoprotein screening - and why? Acta Obstet Gynecol Scand 63(8):687-91.

Bevan, E. G., Chee, L. C., McGhee, S. M. et al. (1993). Patients' attitudes to participation in clinical trails. British Journal of Clinical Pharmacology, 35:204207.

Black, R. B. (1992). Seeing the baby: the impact of ultrasound technology. Journal of Genetic Counseling, 1(1): 45-54.

Bordo, S. (1993). Unbearable Weight: Feminism, Western Culture and the Body. Berkeley: University of California Press.

Boyatzis, R.E. (1998). Transforming qualitative analysis: thematic analysis and code development. Thousand Oaks: Sage.

Braun, V. \& Clarke, V. (2006). Using thematic analysis in psychology. Qualitative research in psychology, 3:77-101.

Brodie, P. (2006). Addressing the barriers to midwifery-Australian midwives speaking out. The Australian Journal of Midwifery, 15(3):5-14. 
Browner, C. H. \& Press, N. (1995). The normalization of prenatal diagnostic testing. In F. Ginsberg \& R. Rapp (eds.), The Politics of Reproduction. Berkeley: University of California Press, pp. 307-322.

Browner, C. H. \& Press, N. (1996). The production of authoritative knowledge in American prenatal care. Medical Anthropology Quarterly, 16:141-156.

Bryman, A. (2008). Social Research Methods ( $3^{\text {rd }}$ edition.). Oxford: Oxford University Press.

Burr, V. (1995). An introduction to social constructionism. London: Routledge.

Burr, V. (2003). Social constructionism (2 ${ }^{\text {nd }}$ edition). London \& New York: Routledge.

Byrne, M. (2001). Understanding life experiences through a phenomenological approach to research. AORN Journal, 73(4):830-832.

Callon, M. \& Rabeharisoa, V. (2004). Gino's lesson on humanity: genetics, mutual entanglements and the sociologist's role. Economy \& Society, 33(1):1-27.

Campbell, N. (2008). New Zealand College of Midwives. Personal communication.

Campbell, S. \& Little, D. (1980). Clinical potential of real-time ultrasound. In M. Bennett \& S. Campbell (eds.), Real-time Ultrasound in Obstetrics. Oxford: Blackwell Scientific Publications.

Capen, K. (1995). New prenatal screening procedures raise spectre of more "wrongful-birth" claims. Canadian Medical Association Journal, 152(5):734736.

Chamberlain, G. \& Steer, P. (eds.) (2001). Turnbull's Obstetrics (3rd edition). London: Churchill Livingstone.

Chang, T. C. (2006). Antenatal screening for Down syndrome in New Zealand: time for a national screening policy? The Royal Australian and New Zealand College of Obstetricians and Gynaecologists, 46: 92-96.

Charmaz, K. (1995). The body, identity, and self: adapting to impairment. The Sociological Quarterly, 36(4):657-680.

Charon, R. (2001a). Narrative medicine: a model for empathy, reflection, profession and trust. Journal of the American Medical Association, 286(15):1897-1902.

Charon, R. (2001b). Narrative medicine: form, function and ethics. Annals of Internal Medicine, 134(1):83-87. 
Chervenak, F. A., McCulloch, L. B. \& Chervenak, J. L. (1989). Prenatal informed consent for sonogram: an indication for obstetric ultrasonography. American Journal of Obstetrics and Gynecology, 161(4):857-60.

Chudleigh, T. (1999). Scanning for pleasure. Ultrasound in Obstetrics \& Gynecology, 14:369-371.

Clarke, A. \& Montini, T. (1993). The many faces of RU486: tales of situated knowledges and technological contestations. Science, Technology \& Human Values, 18:42-78.

Clayton, E. W. (1999). What should be the role of public health in newborn screening and prenatal diagnosis? American Journal of Preventive Medicine, 16(2):111115.

Copelton, D. A. (2007). 'You are what you eat': nutritional norms, maternal deviance, and neutralization of women's prenatal diets. Deviant Behaviour, 28(5):467-494.

Corea, G. (1985). The mother machine: reproductive technologies from artificial insemination to artificial wombs. New York: Harper and Row.

Corrigan, O. (2003). Empty ethics: the problem with informed consent. Sociology of Health \& Illness, 25(3):768-792.

Cowan, R. S. (1994).Women's roles in the history of amniocentesis and chorionic villus sampling. In K. H. Rothenberg, \& E.J. Thompson (eds.), Women and Prenatal Testing: Facing the Challenges of Genetic Technology. Columbus: Ohio State University Press.

Cunningham, F. G., Leveno, K. J., Bloom, S. L., Hauth, J. C., Gilstrap, L. C. \& Wenstrom, K. D. (eds.) (2005). Williams Obstetrics (22nd edition). New York: McGraw-Hill.

Cunningham, W. \& Dovey, S. (2006). Defensive changes in medical practice and the complaints process: a qualitative study of New Zealand doctors. New Zealand Medical Journal, 119(1244):U2283

Cunningham-Burley, S. \& Amos, A. (1998). The new genetics and health: mobilizing lay expertise. Public Understanding of Science, 7(1):41-60.

Cunningham-Burley, S. \& Kerr, A. (1999). Defining the 'social': towards an understanding of scientific and medical discourses on the social aspects of the new human genetics. Sociology of Health \& Illness, 21(5):647-668.

Cussins, C. (1998). Ontological choreography: agency for women patients in an infertility clinic. In M. Berg \& A. Mol (eds.), Differences in Medicine. Durham, NC: Duke University Press, pp. 166-201. 
Dahl, K., Kesmodel, U., Hvidman, L. \& Olesen, F. (2006). Informed consent: providing information about prenatal exams. Acta Obstetricia et Gynecologica Scandinavica, 85(12):1420-1425.

Davis-Floyd, R. (1992). Birth as an American rite of passage. Berkeley: University of California Press.

De Vries, R. (2003). How can we help? From "sociology in" to "sociology of" bioethics. Journal of Law, Medicine \& Ethics, 32:279-292.

Dew, K. \& Kirkman, A. (2002). Sociology of Health in New Zealand. Melbourne: Oxford University Press.

Dinno, M. A., Dyson, M., Young, S. R., Mortimer, A. J., Hart, J. \& Crum, L. A. (1989). The significance of membrane changes in the safe and effective use of therapeutic and diagnostic ultrasound. Physics in Medicine \& Biology, 34:15431552.

Donnai, D. \& Kerzin-Storrar, L. (1991) Counselling after prenatal diagnosis, in J. O' Drife \& D. Donnai (eds.), Antenatal Diagnosis of Fetal Anomalies. Oxford: Springer-Verlag.

Donovan, J. L. \& Blake, D. R. (1992). Patient non-compliance: deviance or reasoned decision-making? Social Science \& Medicine, 34(5):507-513.

Donovan, S. (2006). Inescapable burden of choice? The impact of a culture of prenatal screening on women's experiences of pregnancy. Health Sociology Review, 15(4): 397-405.

Dooley S. L. (1999). Routine ultrasound in pregnancy. Clinical Obstetrics and Gynecology, 42(4):737-748.

Draper, J. (2002). 'It was a real good show': the ultrasound scan, fathers and the power of visual knowledge. Sociology of Health \& Illness, 24(6):771-795.

Duden, B. (1993). Disembodying Women. Massachusetts: Harvard University Press.

Du Plessis, R. (2003). Democracy, participation and 'scientific citizenship': New Zealand initiatives. Paper presented to the Policy and Politics International Conference on 'Policy and Politics in a Globalising World', Bristol, 24-26 July, UK.

Retrieved 23 November 2009 from the World Wide Web:

http://ir.canterbury.ac.nz/bitstream/10092/644/1/12589301_DuPlessisPPfinal.pdf

Durbin, S. A. (1997). Words spoken in a dimly lit room. Journal of Diagnostic Medical Sonography, 13:175-178.

D'Urso, P. S., \& Thompson, R. G. (1998). Fetal Biomodelling. Australian \& New Zealand Journal of Obstetrics and Gynaecology, 38(2): 205-207. 
Dyson, R. L., Pretorius, D. H., Budorick, N. E., Johnson, D. D., Sklansky, M. S., Cantrell, C. J., Lai, S. \& Nelson, T. R. (2000). Three-dimensional ultrasound in the evaluation of fetal anomalies. Ultrasound in Obstetrics \& Gynaecology, $16(4): 321-328$.

Economides, D.L. (1999). Early pregnancy screening for fetal abnormalities. Ultrasound in Obstetrics \& Gynecology, 13:81-83.

El-Wakeel, H., Taylor, G. J. \& Tate, J. J. T. (2006). What do patients really want to know in an informed consent procedure? A questionnaire-based survey of patients in the Bath area, UK. Journal of Medical Ethics, 32:612-616.

English, D. C. (2002). Valid informed consent: a process, not a signature. The American Surgeon, 68(1):45-8.

Essex, C., Counsell, A. \& Geddis, D. C. (1992). The demographic characteristics of early and late attenders for antenatal care. Australian and New Zealand Journal of Obstetrics and Gynaecology, 32:306-8.

Ettore, E. (2000). Reproductive genetics, gender and the body: 'please doctor, may I have a normal body?' Sociology 34(3):403-420.

Ettore, E. (2002). Reproductive Genetics, Gender and the Body. London: Routledge.

Eurenius, K., Axelsson, O, \& Sjoden, P. (1996). Pregnancy, ultrasound screening and smoking attitudes. Gynaecologic \& Obstetric Investigation, 42(2):73-6.

Ewen, S. \& Ewen, E. (1982). Channels of desire: mass images and the shaping of American Consciousness. New York: McGraw-Hill.

Ewigman, B. G., Crane, J. P., Frigoletto, F. D., LeFerve, M. L., Bain, R. P. \& McNellis, D. (1993). Effect of prenatal ultrasound screening on perinatal outcome: the RADIUS study group. New England Journal of Medicine, 171:821827.

Farquhar, D. (1996). The Other Machine: Discourse and Reproductive Technologies. New York \& London: Routledge.

Farsides, B. Williams, C. \& Alderson, P. (2004). Aiming towards "moral equilibrium": health care professionals' views on working within the morally contested field of antenatal screening. Journal of Medical Ethics, 30:505-509.

Fereday, J. \& Muir-Cochrane, E. (2006). Demonstrating rigor using thematic analysis: a hybrid approach of inductive and deductive coding and theme development. International Journal of Qualitative Methods, 5(1). Retrieved October 28, 2007, from the World Wide Web: http:/www.ualberta.ca/iiqm/backissues/5_1/pdf/fereday.pdf. 
Ferreira da Costa, L., Hardy, E., Duarte Osis, M. \& Faundes, A. (2005). Termination of pregnancy for fetal abnormality incompatible with life: women's experiences in Brazil. Reproductive Health Matters, 13(26):139-146.

Filly, R. A. \& Crane, J. P. (2002). Routine obstetric sonography. Journal of Medical Ultrasound, 21:713-718.

Fishman, E. K., Magid, D., Ney, D. R., Chaney, E. L., Pizer, S. M. et al (1991). Three-dimensional imaging. Radiology, 181:321-337.

Fletcher, J. C., \& Evans, M.I.(1983). Maternal bonding in early fetal ultrasound examinations. New England Journal of Medicine, 308:392-393.

Fletcher, J., Hicks, N., Kay, J. \& Boyd, P.(1995).Using decision analysis to compare policies for antenatal screening for Down's syndrome. British Medical Journal, 311:351-356.

Frank, A. (1990). Bringing bodies back in: a decade in review. Theory, Culture \& Society, 7:131-162.

Frank, A. (1995). The Wounded Storyteller: Body, Illness and Ethics. Chicago: University of Chicago Press.

Frank, A. (1997). Illness as moral occasion: restoring agency to ill people. Health, 1(2): 131-148.

Franklin, S. (1991). Fetal fascinations: new dimensions to the medical-scientific construction of fetal personhood. In S. Franklin, C. Lury \& J. Stacey (eds.), Offcentre: Feminism and Cultural Studies. London: Harper Collins, pp. 190-203.

Franklin, S. (1995). Science as culture, cultures of science. Annual Review of Anthropology, 24:163-84.

Franklin, S. (1997). Embodied progress: a cultural account of assisted conception. London \& New York: Routledge.

Fraser, D. M. \& Cooper, M. A. (eds.) (2003). Myles Textbook for Midwives (14th edition). Edinburgh: Churchill Livingstone.

Freeman, L. \& Timperley, H. (2004). Partnership in midwifery care in New Zealand. Midwifery, 20(1):2-14.

Ganiats, T. (1996). Justifying prenatal screening and genetic amniocentesis programs by cost-effectiveness analyses: a re-evaluation. Medical Decision-making, 16:4550 .

Garcia, E., Timmermans, D. R. M., van Leeuwen, E. (2008a). The impact of ethical beliefs on decisions about prenatal screening tests: searching for justification. Social Science \& Medicine, 66(3):753-764. 
Garcia, E., Timmermans, D.R.M. \& van Leeuwen, E. (2008b). Rethinking autonomy in the context of prenatal screening. Prenatal Diagnosis, 28;115-120.

Garcia, J., Bricker, L., Henderson, J., Martin, M., Mugford, M., Nielson, J. \& Roberts, T. (2002). Women's views of pregnancy ultrasound: a systematic review. Birth 29(4):225-250.

Garvey, N. (1997). Feminist poststructuralism and discourse analysis. In M.M. Gergen \& S.W. Davis (eds.), Toward a new psychology of gender: a reader. London: Routledge.

Georges, E. (1996). Fetal ultrasound imaging and the production of authoritative knowledge in Greece. Medical Anthropology Quarterly, 10:157-175.

Getz, L. \& Kirkengen, A. L. (2003). Ultrasound screening in pregnancy: advancing technology, soft markers for fetal chromosomal aberrations, and unacknowledged ethical dilemmas. Social Science \& Medicine, 56:2045-2057.

Goodman, J. M. (2007). Protect yourself! Make a plan to obtain "informed refusal". OBG Management, 19(3):45-50. Retrieved June 25, 2008, from the World Wide Web: http://www.obgmanagement.com/article_pages.asp?AID=4848\&UID.

Graham, W., Smith, P., Kamal, A., Fitzmaurice, A., Smith, N. \& Hamilton, N. (2000). Randomised controlled trial comparing effectiveness of touch screen system with leaflet for providing women with information on prenatal tests. British Medical Journal, 320:155-160.

Green, J. \& Statham, H. (1996). Psychosocial aspects of prenatal screening and diagnosis. In T. Marteau \& M. Richards (eds.), The Troubled Helix: Social and Psychological Implications of the New Human Genetics. Cambridge: Cambridge University Press, pp. 140-163.

Green, J., Hewison, J., Bekker, H., Bryant, L \& Cuckle, H. (2004). Psychosocial aspects of genetic screening of pregnant women and newborns: a systematic review (executive summary). Health Technology Assessment, 8(33).

Gudex, C., Nielsen, B. L. \& Madsen, M. (2006). Why women want prenatal ultrasound in normal pregnancy. Ultrasound in Obstetrics and Gynecology, 27:145-150.

Guilliland, K. \& Pairman, S. (1995). The midwifery partnership: a model for practice. In monograph series 95/1, Department of Nursing \& Midwifery. Wellington: Victoria University of Wellington.

Haimes, E. (2002). What can the social sciences contribute to the study of ethics? Theoretical, empirical and substantive considerations. Bioethics, 16(2):89-113.

Hall, S., Bobrow, M. \& Marteau, T.M. (2000). Psychological consequences for parents of false negative results on prenatal screening for Down syndrome: retrospective interview study. British Medical Journal, 320:407-412. 
Harris, G., Connor, L., Bisits, A. and Higgenbotham, N. (2004). "Seeing the baby": pleasures and dilemmas of ultrasound technologies for primiparous Australian women. Medical Anthropology Quarterly, 18(1):23-47.

Health and Disability Commissioner (1996). Code of health and disability services consumers' rights. Auckland: Health and Disability Commissioner.

Helen, I. (2002). Risk and anxiety: polyvalence of ethics in high-tech antenatal care. Critical Public Health, 12(2):119-137.

Helen, I. (2004). Technics over life: risk, ethics and the existential condition in hightech antenatal care. Economy \& Society, 33(1):28-51

Heyman, B., Hundt, G., Sandall, J., Spencer, K., Williams, C., Grellier, R. \& Pitson, L. (2006). On being at higher risk: A qualitative study of prenatal screening for chromosomal anomalies. Social Science \& Medicine, 62:2360-2372.

Irving, L. (2004). Nuchal translucency screening for Down's syndrome: the midwife's role. New Zealand College of Midwives Journal, 31:18-21.

Ji, E. K., Pretorius, D. H., Newton, R., Uyan, K., Hull, A. D., Hollenbach, K. \& Nelson, T. R. (2005). Effects of ultrasound on maternal bonding: a comparison of two- and three-dimensional imaging. Ultrasound in Obstetrics \& Gynecology, 25:473-477.

Johanson, R., Newburn, M. \& Macfarlane, A. (2002). Has the medicalisation of childbirth gone too far? British Medical Journal, 324(7342):892-895.

Johnson, K. (2003). Keepsake ultrasounds: medicine or marketing? Fetal imaging obstetrics. OB. Gyn. News. Retrieved 1 April 2008 from the World Wide Web: http://findarticles.com/p/articles/mi_mOCYD/is_16_38/ai_107275716.

Johnson, N. P., Lewis, J. \& Ansell D. A. (1995). Does ethnicity influence obstetric intervention? New Zealand Medical Journal, 108(1013):511-2.

Jones, D., King, M. \& Whitaker, M. (2009). Who gets born? How did New Zealand's Bioethics Council arrive at its recommendations? New Zealand Medical Journal, 122(1294):84-91.

Jonsen, A. R., Siegler, M. \& Winslade, W. J. (2006). Clinical ethics: a practical approach to ethical decisions in clinical medicine. New York: McGraw-Hill.

Jorgensen, F. (1995). Declining an alpha-fetoprotein test in pregnancy, why and who? Acta Obstetrcia et Scandinavica, 74:3-11.

Kennell, J. H., Jerauld, R., Wolf, H., Chesler, D., Kreger, N. C., McAlpine, W., Steffa, M, \& Klaus, M. H. (1974). Maternal behaviour one year after early and extended post-partum contact. Developmental Medicine \& Child Neurology, 16(2):172-9. 
Kerr, A, Cunningham-Burley, S. and Amos, A. (1998). Drawing the line: an analysis of lay people's discussions about the new genetics. Public Understanding of Science, 7:113-33.

Kerr, A. \& Cunningham-Burley, S. (2000). On ambivalence and risk: reflexive modernity and the new human genetics. Sociology, 34(2):283-304.

Kieler, H., Ahlsten, G., Haglund, B., Salvesen, K., \& Axelsson, O. (1998). Routine ultrasound screening in pregnancy and aspects of the children's subsequent neurological development. Obstetrics \& Gynecology, 91:750-756.

King, A. (2001). The Primary Health Care Strategy. Wellington: Ministry of Health.

Klaus, M. H., Jerauld, R., Kreger, N. C., McAlpine, W., Steffa, M. \& Kennell, J. H. (1972). Maternal attachment: importance of the first postpartum days. New England Journal of Medicine, 286:460-463.

Kohut, R. J., Dewey, D. \& Love, E. J. (2002). Women's knowledge of prenatal ultrasound and informed choice. Journal of Genetic Counselling, 11(4):265-276.

Kolker, A. \& Burke, B. M. (1994). Prenatal Testing: A Sociological Perspective. Westport, CT: Bergin \& Garvey.

Kurjak, A., Vecek, N., Hafner, T., Funduk-Kurjak, B. \& Ujevic, B. (2002). Prenatal diagnosis: what does four-dimensional ultrasound add? Journal of Perinatal Medicine, 30(1):57-62.

Kvale, S. (1996). InterViews: an introduction to qualitative research interviewing. Thousand Oaks: Sage.

Lawson, K. (2003). Perceptions of deservedness of social aid as a function of prenatal diagnostic testing. Journal of Applied Social Psychology, 33(1):76-90.

Layng, J. (1998). Screening for nuchal translucency: counselling should be considered an integral part of screening programmes. British Medical Journal, 317:749.

Levi, S. \& Chervenak, F. A. (1998). Ultrasound screening for fetal anomalies: is it worth it? Annals of the New York Academy of Sciences, 847:256)

Lewando-Hundt, G., Shoham-Vardi, I., Beckerleg, S., Belmaker, I., Kassem, F., and Abu Jaafar, A. (2001). Knowledge, action and resistance: the selective use of pre-natal screening among Bedouin women of the Negev, Israel. Social Science \& Medicine, 52(4):561-569.

Liamputtong, P., Halliday, J. L., Warren, R., Watson, L. F., and Bell, R. J. (2003) Why do women decline prenatal screening and diagnosis? Australian women's perspective. Women and Health, 37:89-108. 
Lippman, A. (1991). Prenatal genetic testing and screening: constructing needs and reinforcing inequities. American Journal of Law and Medicine, 17(1-2):15-50.

Lippman, A. (1992). Led (astray) by genetic maps: the cartography of the human genome and health care. Social Science \& Medicine, 35:1469-76.

Lippman, A. (1994). The genetic construction of prenatal testing: choice, consent or conformity for women? In Rothenberg, K. H. \& Thomson, E.J. (eds.), Women and Prenatal Testing: Facing the Challenges of Genetic Technology. Columbus OH: Ohio State University Press, pp. 9-33.

Lippman, A. (1999a). Choice as a risk to women's health. Health, Risk \& Society, 1(3):281-291.

Lippman, A. (1999b). Embodied knowledge and making sense of prenatal diagnosis. Journal of Genetic Counselling, 8(5):255-274.

Lopez, J. (2004). How sociology can save bioethics ... maybe. Sociology of Health \& Illness, 26(7):875-896.

Low, P., Paterson, J., Wouldes, T., Carter, A., Williams, M. \& Percival, T. (2005).

Factors affecting antenatal care attendance by mothers of Pacific infants living in New Zealand. New Zealand Medical Journal, 118(1216):U1489.

Lupton, D. (1992). Discourse analysis: a new methodology for understanding the ideologies of health and illness. Australian Journal of Public Health, 16:145-50.

Lupton, D. (1993). Risk as moral danger: the social and political functions of risk discourse in public health. International Journal of Health Services, 23(3):42535 .

Lupton, D. (1995). The Imperative of Health: Public Health and the Regulated Body. London: Sage.

Lupton, D. (1997). Consumerism, reflexivity and the medical encounter. Social Science \& Medicine, 4:373-81.

Lupton, D. (1999). Risk and the ontology of pregnant embodiment. In D. Lupton (ed.), Risk and Sociocultural Theory: New Directions and Perspectives. Cambridge: Cambridge University Press, pp. 59-85.

Lupton, D. (2003). Medicine as Culture: Illness, Disease and the Body in Western Societies (2nd edition). London: Sage.

Maher, J. M. (2002). Visibly pregnant: toward a placental body. Feminist Review, 72: 95-107.

Mansfield, C., Hopfer, S., \& Marteau, T. M. (1999). Termination rates after prenatal diagnosis of Down syndrome, spina bifida, anencephaly, and Turner and 
Kleinfelter syndromes: a systematic literature review. Prenatal Diagnosis, 19:808-812.

Markens, S., Browner, C. H. \& Press, N. (1997). Feeding the fetus: on interrogating the notion of maternal-fetal conflict. Feminist Studies, 23:351-372.

Markens, S., Browner, C. H. \& Press, N. (1999). 'Because of the risks': how US pregnant women account for refusing prenatal screening. Social Science and Medicine, 49(3):359-369.

Marshall, G. (1998). Oxford Dictionary of Sociology. Oxford \& New York: Oxford University Press.

Marteau, T.M.(1989). Psychological costs of screening. British Medical Journal, 299:527.

Marteau, T. M., Slack, J., Kidd, J. \& Shaw, R. W. (1992). Presenting a routine screening test in antenatal care: practice observed. Public Health, 106(2):131141.

Marteau, T. M., Cook, R., Kidd, J., Michie, S., Johnston, M., Slack, J. \& Shaw, R. W. (1992). The psychological effects of false-positive results in prenatal screening for fetal abnormality: a prospective study. Prenatal Diagnosis, 12:205-214.

Marteau, T. M. (1993). Psychological consequences of screening for Down's syndrome. British Medical Journal, 307: 146-7.

Marteau, T. M., Drake, H. \& Bobrow, M. (1994). Counselling following diagnosis of a fetal abnormality: the differing approaches of obstetricians, clinical geneticisists, and genetic nurses. Journal of Medical Genetics, 31:864-867.

Marteau, T. M. (1995).Towards informed decisions about prenatal testing: a review. Prenatal diagnosis, 15:1215-1226.

Marteau, T. M. and Drake, H. (1995). Attributions for disability: the influence of genetic screening. Social Science and Medicine, 40(8):1127-1132.

Marteau, T.M., \& Dormandy, E. (2001). Facilitating informed choice in prenatal testing: how well are we doing? American Journal of Medical Genetics, 106:185190.

McCarthy, J. (2003). Principalism or narrative ethics: must we choose between them? Medical Humanities, 29:65-71.

McFadyen, A., Gledhill, J., Whitlow, B., \& Economides, D. (1998). First trimester ultrasound screening carries ethical and psychological implications. British Medical Journal, 317(7160):694-695.

McKinlay, J.B. (1982). From "promising report" to "standard procedure": seven stages in the career of a medical innovation. In J.B. Millbank (ed.), Technology 
and the future of health care, Milbank Reader, 8:233-270. Cambridge, MA: MIT Press.

McNay, M. B. \& Fleming, J. E. E. (1998). Forty years of obstetric ultrasound 19571997: from A-scope to three dimensions. Ultrasound in Medicine \& Biology 25(1):3-56.

Medforth, J., Battersby, S., Evans, M., Marsh, B. \& Walker, A. (2006). Oxford Handbook of Midwifery. Oxford University Press.

Merz, E. (1997). Current technical possibilities of 3D ultrasound in gynecology and obstetrics. Ultraschall Med 18:190-195.

Mitchie, S., Drake, H., Bobrow, M. \& Marteau, T. (1995). A comparison of public and professionals' attitudes towards genetic developments. Public Understanding of Science, 4:243-253.

Milligan, E. (2004). Ethical practice in prenatal screening - can informed consent deliver? In Bailey, C., Cabrera, D. \& Buys, L. (eds.), Proceedings, Social Change in the 21st Century Conference. Centre for Social Change Research, Queensland University of Technology. Retrieved 19 February 2007 from the World Wide Web: http://eprints.qut.edu.au/637/1/milligan-eleanor.pdf

Ministy of Health New Zealand (2001). Primary health care strategy. Wellington: Minstry of Health.

Ministry of Health New Zealand (2002). Patient information pamphlet. Your pregnancy/To haputanga. Retrieved 7 April 2006 from the World Wide Web: http://www.healthed.govt.nz/uploads/docs/HE1420.pdf

Ministry of Health New Zealand (2002). Maternity Services Notice Pursuant to Section 88 of the Health and Disability Act 2000. New Zealand.

Ministry of Health New Zealand (2007). Amendment to Maternity Services Notice Pursuant to Section 88 of the Health and Disability Act 2000. New Zealand.

Mitchell, L. (2004). Women's experiences of unexpected ultrasound findings. Journal of Midwifery \& Women's Health, 49(3):228-234.

Mitchell, L. M. \& Georges, E. (1997). Cross-cultural cyborgs: Greek and Canadian women's discourses on fetal ultrasound. Feminist Studies, 23(2):373-401.

Mitchell, L. M. \& Georges, E. (1998). Baby's first picture: the cyborg fetus of ultrasound imaging. In Davis-Floyd, R. \& Dumit, J. (eds.), Cyborg Babies: From Techo-sex to Techno-tots. New York: Routledge, pp. 105-124.

Mol, A. (1998). Lived reality and the multiplicity of norms: a critical tribute to George Canguilhem. Economy \& Society, 27(2):274-284. 
Mol, A. (2002). The Body multiple: ontology in medical practice. Durham, NC and London: Duke University Press.

Mongelli, M., Wilcox, M. \& Gardosi, J. (1996). Estimating the date of confinement: Ultrasonographic biometry versus certain menstrual dates. American Journal of Obstetrics \& Gynecology, 174(1):278-281.

Morris, J. K., Wald, N. J. \& Watt, H. C. (1999). Fetal loss in Down syndrome pregnancies. Prenatal diagnosis, 19:142-145.

Muller, M. (1996). Prenatal and postnatal attachment: a modest correlation. Journal of Obstetric and Neonatal Nursing, 25(2):161-6.

National Health Committee (1999). Review of Maternity Services. Wellington. National Health Committee.

National Council on Radiation Protection and Measurements (NCRP) (2002). Exposure criteria for medical diagnostic ultrasound: II. Criteria based on all known mechanisms. NCRP report no. 140 (summary). Bethesda, MD: NCRP.

National Screening Advisory Committee, (2007). Advice to the Director-General of Health: Antenatal Down syndrome screening. Retrieved October 52007 from the World Wide Web:

http://www.nsu.govt.nz/Files/Strategic_Screening/NSAC_Advice_to_DG__Antenatal_screening_for_Down_Syndrome.pdf

National Screening Unit (NSU) (2006). Media release, June 8, 2006. 'Down syndrome antenatal screening under review'. Retrieved August 152006 from the World Wide Web:

http://www.moh.govt.nz/moh.nsf/UnidPrint/MH4824?OpenDocument

National Screening Unit (New Zealand Ministry of Health) (2007a). Antenatal Down Syndrome Screening in New Zealand 2007: A Report of the Antenatal Down Syndrome Screening Advisory Group to the National Screening Unit. Wellington: National Screening Unit.

National Screening Unit (NSU) (2007b). Media release, April 26, 2007. 'Report on antenatal Down syndrome released by the National Screening Unit'. Retrieved 12 May 2007 from the World Wide Web: http://www.nsu.govt.nz/News/2291.asp

National Screening Unit (NSU) (2008) website. 'Achieving our vision'. Retrieved 4 June 2008 from the World Wide Web: http://www.nsu.govt.nz/About/772.asp

National Screening Unit (NSU) (2009). Information on maternal serum screening.

Retrieved 11 January from the World Wide Web:

http//www.nsu.govt.nz/Files?ANNB/Maternal_Serum_Test_Consumer_Info_She et_FINAL.pdf

Nelson, T. R., Downey, D. B., Pretorius, D. H. \& Fenster, A. (1999). Threedimensional Ultrasound. Philadelphia: Lippincott, Williams \& Wilkins. 
Nettleton, S. (1995). The Sociology of health and Illness. Oxford: Polity.

Newnham, J. P., Evans, S. F., Michael, C. A., Stanley, F. J. \& Landau, L. (1993). Effects of frequent ultrasound during pregnancy: a randomized controlled trial. Lancet, 342:887-891.

New Zealand College of Midwives (NZCOM) (1995). Consensus statement Ultrasound. Retrieved 7 April 2006 from the World Wide Web:

http://www.midwife.org.nz/index.cfm/3,108,557/ultrasound-1995-referencesupdated-2002-.pdf

New Zealand College of Midwives (NZCOM) (2009).Midwifery information. Retrieved 3 April 2009 from the World Wide Web: http://www.midwife.org.nz/index.cfm/1,100,html.

New Zealand Herald, 17/09/05. 'Rise in Down syndrome babies blamed on lack of screening'. Auckland: APN Holdings NZ, Ltd.

Nicholaides, K. H., Azar, G., Byrne, D. et al. (1992). Fetal nuchal translucency: ultrasound screening for chromosomal defects in first trimester of pregnancy. British Medical Journal, 304(6831):867-869.

Nicholaides, K. H., Spencer, K., Avgidou, K., Faiola, S., and Falcon, O. (2005). Multicenter study of first trimester screening for trisomy 21 in 75, 821 pregnancies: results and estimation of the potential impacts of individual riskorientated two-stage first-trimester screening. Ultrasound in Obstetrics \& Gynaecology, 25(3):221-266.

Nicholaides, K. H., Chervenak, F. A., McCulloch, L. B., Avgidou, K. \& Papageorghiou, A. (2005). Evidence-based obstetrics ethics and informed decision-making by pregnant women about invasive diagnosis after firsttrimester risk assessment for risk of trisomy 21. American Journal of Obstetrics and Gynecology, 193(2):322-326.

Nyberg, D. A., Mahony, B. S. \& Pretorius, D. H. (eds.) (1990). Diagnostic Ultrasound of Fetal Anomalies. St Louis, MO: Mosby Books.

Nyberg, D. A., McGahan, J. P., Pretorius, D. H. and Pilu, G. (eds.) (2003). Diagnostic Imaging of Fetal Anomalies. Philadelphia: Lippincott, Williams \& Wilkins.

O’Leary, P., Breheny, N., Reid, G., Charles, T. \& Emery, J. (2006). Regional variations in prenatal screening across Australia: Stepping towards a national policy framework. Australian and New Zealand Journal of Obstetrics \& Gynaecology, 46:427-432.

Oakley, A. (1984). The Captured Womb: A History of the Medical Care of Pregnant Women. Oxford and New York: Blackwell.

Oakley, A. (1986). The history of ultrasonography in obstetrics. Birth, 13(s1):5-10. 
Oakley, M.E. (1997). Women's Subjective Experience of the Ultrasound Examination During Pregnancy. Unpublished MA thesis (health sciences). Dunedin: Otago University.

Oliver, S., Rajan, L., Turner, H., Oakley, A., Entwhistle, I., Watt, T., Sheldon, T.A. \& Rosser, J. (1996). Informed choice for users of health services: views on ultrasonography leaflets of women in early pregnancy, midwives, and ultrasonographers. British Medical Journal, 313(7067):1251-3.

Ozturk, K. (2008). Independent midwife, Domino Midwifery Group, Wellington. Personal communication.

Pairman, S. \& Guilliland, K. (2003). Developing a midwife-led maternity service: the New Zealand experience. In Kirkham, M. (ed.), Birth Centres: A Social Model for Maternity Care. London: Elsevier Science Ltd.

Pairman, S. (2006). Midwifery partnership: working 'with' women. In Page, L. \& McCandlish, R. (eds.), The New Midwifery: Science and Sensitivity in Practice. Edinburgh: Churchill Livingstone, pp. 73-94.

Parens, E. \& Asch, A. (1999). The disability rights critique of prenatal genetic testing: reflections and recommendations. Hastings Center Report, 29:S1-S22.

Paterson, R. (2006). National antenatal screening programme for Down syndrome response of the Health and Disability Commissioner. Retrieved 25 November 2009 from the World Wide Web:

http://www.hdc.org.nz/files/hdc/publications/screening-for-down-syndrome.pdf

Paul, K. \& Nawrocki, C. (1997). Prenatal ultrasound videos: entertainment or excess? Journal of Diagnostic Medical Sonography, 13:309-312.

Payne, G. \& Payne, J. (2004). Key Concepts in Social Research. London: Sage.

Pennington, J. (2005). From deficit to democracy: dialogue, deliberation and public participation in the biosciences. Ministry for the Environment, Wellington.

Pershutte, W. H. (1995). Failure to address the psychosocial benefit of prenatal sonography - another failing of the RADIUS study. Journal of Ultrasound in Medicine, 14:795-796.

Petchesky, R. P. (1987). Fetal images: the power of visual culture in the politics of reproduction. Feminist Studies, 13(2):263-292.

Petchesky, R. P. (1990). Abortion and Woman's Choice: The State, Sexuality, and Reproductive Freedom. Boston: Northeastern University Press.

Petersen, A. \& Lupton, D. (1996). The New Public Health: Health and Self in an Age of Risk. St Leonards, NWS: Allen \& Unwin. 
Petersen, A. \& Bunton, R. (2002). The New Genetics and the Public's Health. London: Routledge.

Pilnick, A., Fraser, D. \& James, D. (2004). Presenting and discussing nuchal translucency screening for fetal abnormality in the UK. Midwifery, 20(1): 82-93.

Potter, J. \& Wetherell, M. (1987). Discourse and social psychology: beyond attitudes and behaviour. London: Sage.

Potts, A., Gavey, N., Grace, V. \& Vares, T. (2003). The downside of Viagra: women's experiences and concerns. Sociology of Health \& Illness, 25(7):697719.

Press, N. \& Browner, C. H. (1994). Collective silences, collective fictions: how prenatal diagnostic testing became part of routine prenatal care. In K. H. Rothenberg \& E. J. Thomson (eds.), Women and Prenatal Testing: Facing the Challenges of Genetic Technology. Columbus: Ohio State University Press, pp. 201-18.

Press, N. \& Browner, C. H. (1995). Risk, autonomy and responsibility: informed consent for prenatal testing. Hastings Center Report, 25(3): S9-S12. Retrieved 25 June, 2008, from the World Wide Web: http://www.jstor.org/stable/3562123.

Press, N. \& Browner, C. H. (1996). The production of authoritative knowledge in American prenatal care. Medical Anthropology Quarterly, 10(2):141-156.

Press, N. \& Browner, C. H. (1997). Why women say yes to prenatal diagnosis. Social Science \& Medicine, 45(7):979-989.

Press, N. \& Browner, C. H. (1998). Characteristics of women who refuse an offer of prenatal diagnosis: data from the California maternal serum alpha fetoprotein blood test experience. American Journal of Genetics, 78:433-445.

Press, N., Browner, C. H., Tran, D., Morton, C. \& le Master, B. (1998). Provisional normalcy and "perfect babies": pregnant women's attitudes towards disability in the context of prenatal testing. In S. Franklin \& H. Ragone (eds.), Reproducing Reproduction: Kinship, Power and Technological Innovation. Philadelphia: University of Pennsylvania Press, pp. 46-65.

Press, N. \& Browner, C. H. \& Markens, S. (1999). 'Because of the risks': how U.S. women account for refusing prenatal screening. Social Science \& Medicine, 49(3):359-369.

Pretorius, D. H. \& Mahony, B. S. (1990). The role of obstetrical ultrasonography. In D. A. Nyberg, B.S. Mahony \& D. H. Pretorius (eds.), Diagnostic Ultrasound of Fetal Anomalies. St Louis, MO: Mosby Books.

Pretorius, D. H., Nelson, T. R. \& James, G. (2003). Three-dimensional ultrasound in obstetrics. In D. A. Nyberg, J. P. McGahan, D. H. Pretorius \& G. Pilu (eds.), 
Diagnostic Imaging of Fetal Anomalies. Philadelphia: Lippincott, Williams \& Wilkins, pp. 969-988.

Proud, J. \& Murphy-Black, T. (1997). Choice of a scan: how much information do women receive before ultrasound? British Journal of Midwifery, 5(3):144-147.

Rados, C. (2004). FDA cautions against ultrasound 'keepsake' images. FDA Consumer Magazine, January/February 2004. Retrieved14 May, 2009, from the World Wide Web: http://www.fda.gov/FDAC/features/2004/104_images.html.

Rapp, R. (1987). Moral pioneers: women, men and fetuses on a frontier of reproductive technology. Women \& Health, 13:101-116.

Rapp, R. (1998). Refusing prenatal diagnosis: the meanings of bioscience in a multicultural world. Science, Technology \& Human Values, 23(1):45-70.

Rapp, R. (1999a). Testing Women, Testing the Fetus: The Social Impact of Amniocentesis in America. New York: Routledge.

Rapp, R. (1999b). One new reproductive technology, multiple sites: how feminist methodology bleeds into everyday life. In, Clarke, A.E. \& Olesen, V.L. (eds.), Revisioning women, health and healing: feminist, cultural and technoscience perspectives, pp.119-134.

Raynor, B. D. (2003). Routine Ultrasound in Pregnancy. Clinical Obstetrics \& Gynaecology, 46(4):882-89

Raz, A. (2004). "Important to test, important to support": attitudes toward disability rights and prenatal diagnosis among leaders of support groups for genetic disorders in Israel. Social Science \& Medicine, 59:1857-1866.

Reiger, K. \& Dempsey, R. (2006). Performing birth in a culture of fear: an embodied crisis of late modernity. Health Sociology Review, 15(4):364-373.

Ridley, D. (2001). Informed consent, informed refusal, informed choice - what is it that makes a patient's medical treatment decisions informed? Medicine \& Law, 20(2):205-214.

Riddle, B. (2005). Normative practices and normative identities: A critical feminist investigation of pregnancy ultrasound. Unpublished doctoral thesis (clinical psychology), Duquesne University, Pennsylvania USA.

Romano, P. S. \& Waitzman, N. J. (1998). Can decision analysis help us decide whether ultrasound screening for fetal anomalies is worth it? Annals of the New York Academy of Sciences, 847(1):154-172.

Rothman, B. K. (1986). The tentative pregnancy: Prenatal diagnosis and the future of motherhood. New York: Viking. 
Royal Australian and New Zealand College of Obstetricians and Gynaecologists

(RANZCOG) (2006). Joint HGSA/RANZCOG Prenatal diagnosis policy (C-Obs

5). Retrieved 25 March, 2007 from the World Wide Web:

http://www.ranzcog.edu.au/publications/statements/C-obs5.pdf.

Royal Australian and New Zealand College of Obstetricians and Gynaecologists

(RANCOG) (2006). Patient information leaflet. Prenatal screening tests for

Down syndrome and other fetal conditions. Camberwell, VIC: Mi-tec Medical

Publishing.

Royal Australian and New Zealand College of Obstetricans and Gynaecologists

(RANZCOG) (2007). Statement on prenatal screening tests for Trisomy 21

(Down syndrome), Trisomy 18 (Edwards syndrome) and neural tube defects (C-

Obs 4). Retrieved March 102008 from the World Wide Web:

http://www.ranzcog.edu.au/publications/statements/C-obs4.pdf

Royal Australian and New Zealand College of Obstetricians and Gynecologists

(RANZCOG) (2009) Statement on Home Births (C-Obs 2). Retrieved 1

September 2009 from the World Wide Web:

http://www.ranzcog.edu.au/publications/statements/C-obs2.pdf

Royal Australian and New Zealand College of Obstetricians and Gynaecologists

(RANZCOG) (2008) Statement on Antenatal Screening Tests (C-Obs 3).

Retrieved 13 May 2008 from the World Wide Web:

http://www.ranzcog.edu.au/publications/statements/C-obs3.pdf

Rudolfsdottir, A. G. (2000). 'I am not a patient, and I am not a child': the institutionalization and experience of pregnancy. Feminism \& Psychology, 10(3):337-350.

Ruiz, M. A. \& Murphy, K. (1992). Sonographer-fetus bonding. Journal of Diagnostic Medical Sonography, 8:269-275.

Rustico, M. A., Mastromatteo, C., Grigio, M., \& Nicolini, U. (2005). Twodimensional vs. two-plus four-dimensional ultrasound in pregnancy and the effect on maternal emotional status: a randomized study. Ultrasound in Obstetrics \& Gynecology, 25:468-472.

Sadler, L., McCowan, L., \& Stone, P. (2002). Associations between ethnicity and obstetric intervention in New Zealand. New Zealand Medical Journal, 115(1147):36-9.

Santalahti, P., Aro, A. R., Hemminki, E., Helenius, H. \& Ryynanen, M. (1998). On what grounds do women participate in prenatal screening? Prenatal Diagnosis, 18:153-165.

Sandelowski, M. (1994a).Channel of desire: fetal ultrasonography in two usecontexts. Qualitative Health Research, 4(3):262-280. 
Sandelowski, M (1994b). Focus on qualitative methods: the use of quotes in qualitative research. Research in Nursing \& Health, 17:479-482.

Sandelowski, M. (1998). Looking to care or caring to look? Technology and the rise of spectacular nursing. Holistic Nursing Practice, 12(4):1-11.

Sandelowski, M. (2000a). Focus on research methods: whatever happened to qualitative description? Research in Nursing \& Health, 23:334-340.

Sandelowski, M. (2000b) 'This most dangerous instrument': propriety, power, and the vaginal speculum. Journal of Obstetric, Gynecologic, \& Neonatal Nursing, 29(1): 73-82.

Sandelowski, M. (2002). Reembodying qualitative enquiry. Qualitative Health Research, 12:104-115.

Sandelowski, M. \& Jones, L. C. (1996). 'Healing fictions': stories of choosing in the aftermath of the detection of fetal anomalies. Social Science \& Medicine, 42(3):353361.

Sandmire, H.F. (1990). Whither electronic fetal monitoring? Obstetrics \& Gynaecology, 76(6):1130-1134.

Sanger, C. (2008). Seeing and believing: mandatory ultrasound and the path to protected choice. UCLA Law Review, 56:351-408.

Santalahti, P., Aro, A.R., Hemminki, E., Helenius, H. \& Ryynanen, M. (1998). On what grounds do women participate in prenatal screening? Prenatal diagnosis, 18(2):153-165.

Sawicki, J. (1991). Disciplining Foucault: Feminism, Power and the Body. New York: Routledge.

Scharf, A., Ghazwiny, M. F., Steinborn, A., Baier, P. \& Sohn, C. (2001). Evaluation of two-dimensional versus three-dimensional ultrasound in obstetric diagnostics: a prospective study. Fetal Diagnosis \& Therapy, 16(6):333-341.

Scoop Independent News (2006). Media release. 'DPA disputes antenatal screening claims'. Retrived 3 September 2006 from the World Wide Web: http://www.scoop.co.nz/stories/GEO606/500095.htm

Scully, J. L., Rippberger, C. \& Rehmann-Sutter, C. (2004). Non-professionals' evaluations of gene therapy ethics. Social Science \& Medicine, 58:1415-1425.

Scully, J. L., Banks, S., \& Shakespeare, T. (2006a). Chance, choice and control: lay debate on prenatal social sex selection. Social Science \& Medicine, 63:21-31.

Scully, J. L., Shakespeare, T. \& Banks, S. (2006b). Gift not commodity? Lay people deliberating social sex selection. Sociology of Health \& Illness, 28(6):749-767. 
Scully, J. L., Porz, R. \& Rehmann-Sutter, C. (2007). You don't make genetic test decisions from one day to the next: using time to preserve moral space. Bioethics, 21(4):208-217.

Sedgmen, B., McMahon, C., Cairns, D., Benzie, R. J. \& Woodfield, R. L. (2006). The impact of two-dimensional versus three-dimensional ultrasound exposure on maternal-fetal attachment and maternal health behaviour in pregnancy. Ultrasound in Obstetrics \& Gynecology, 27:245-251.

Shakespeare, T. (1998). Choices and rights: eugenics, genetics and disability equality. Disability \& Society, 13(5):665-681.

Shakespeare, T (1999). 'Losing the plot?'Medical and activist discourses of contemporary genetics and disability. Sociology of Health \& Illness, 21(5):669688 .

Shildrick, M. (1997). Leaky Bodies and Boundaries: Feminism, Postmodernism and (Bio)ethics. London \& New York: Routledge.

Shildrick, M. \& Price, J. (eds.) (1999). Feminist Theory and the Body. Edinburgh: Edinburgh University Press.

Singer, P. A., Pellegrino, E. D. \& Siegler, M. (2001). Clinical ethics revisited. BMC Medical Ethics, 2:1.

Silverman, D. (1993). Interpreting qualitative data: methods for analyzing talk, text and interaction. London: Sage.

Smeltzer, S. (1994). The concerns of pregnant women with Multiple Sclerosis. Qualitative Health Research, 4:481-502.

Smith-Bindman, R., Chu, P., Bacchetti, P., Waters, J., Mutton, D. \& Alberman, E. (2003). Prenatal screening for Down syndrome in England and Wales and population-based birth outcomes. American Journal of Obstetrics and Gynecology, 189(4):980-985.

Stake, R. (2000). Case studies. In N. Denzin \& Y. Lincoln, (eds.), Handbook of Qualitative research (2nd edition). London: Sage.

Stake, R. (2005) Qualitative case studies. In N.K. Denzin \& Y.S. Lincoln (eds.), The Sage handbook of qualitative research ( $3^{\text {rd }}$ edition). Thousand Oaks, CA: Sage, pp.433-466.

Statham, H. \& Green, J. (1993). Serum screening for Down's syndrome: some women's experiences. British Medical Journal, 307(6897):174-176.

Statham, H. \& Green, J. (1996). Psychosocial aspects of prenatal screening and diagnosis. In T. Marteau, \& M. Richards, (eds.), The troubled helix: social and psychological implications of the new human genetics. Cambridge: Cambridge University Press. 
Stephens, M. B., Montefalcon, R. \& Lane, D. A. (2000). The maternal perspective on prenatal ultrasound. Journal of Family Practice, 49:601-4.

Stone, P. \& Austin, D. (2006). Report to the National Screening Unit: Assessment of Antenatal Screening for Down Syndrome in New Zealand. Auckland: Uniservices Ltd.

Stone, P. (2005). Prenatal testing for aneuploidy in New Zealand: time for action. New Zealand Medical Journal, 118:U1545.

Storch, J. \& Kenny, N. (2007). Shared moral work of nurses and physicians. Nursing Ethics, 14(4):478-491.

Tankard-Reist, M. (2006) Defiant birth: women who resist medical eugenics. Melbourne: Spinifex Press.

Tarsa, M., Pretorius, D., D’Agostini, D. (2006). 3-dimensional obstetric ultrasound: tips of the trade. Ultrasound Clinics, 1(2):321-334.

Taylor, J. S. (1992). The public fetus and the family car: from abortion politics to a Volvo advertisement. Public Culture, 4(2):67-80.

Taylor, J. S. (1998). Image of contradiction: obstetrical ultrasound in American culture. In S. Franklin \& H. Ragone (eds.), Reproducing Reproduction: Kinship, Power and Technological Innovation. Philadelphia: University of Pennsylvania Press, pp. 17-45.

Taylor, J. S. (2000). Of sonograms and baby prams: prenatal diagnosis, pregnancy, and consumption. Feminist Studies, 26(2):391-418.

Taylor, J. S. (2002). The public life of the fetal sonogram and the work of the sonographer. Journal of Diagnostic Medical Sonography, 18(367-379).

Tew, M. \& Damstra-Wijmenga, S. M. (1991). Safest birth attendants: recent Dutch evidence. Midwifery, 7:55-63.

Thomas, C. (1997). The baby and the bath water: disabled women and motherhood in social context. Sociology of Health \& Illness, 19(5):622-643.

Thompson, C. (2005). Making Parents: The Ontological Choreography of Reproductive Technologies. Cambridge, MA: MIT Press.

Tiedje, L. (2000). Moral distress in perinatal nursing. Journal of Perinatal Nursing, 14(2):36-43.

Tierney, W.G. (1995). (Re)presentation and voice. Qualitative Inquiry, 1:379-390.

Toi te Taiao/New Zealand Bioethics Council. (2007). Pre-Birth Testing. 
Retrieved 2 February 2008 fro the World Wide Web:

http://www.bioethics.org.nz/downloads/pre-birth-testing.pdf

Toi te Taiao/New Zealand Bioethics Council. (2007). Terms of Reference.

Retrieved 11 January 2010 from the World Wide Web:

http//www.bioethics.org.nz/about-us/terms-of-ref-english.html

UK National Screening Committee Programmes Directorate (2004). National Down's Syndrome Screening programme for England: a Handbook for Staff. Retrieved 14 May 2008 from the World Wide Web: http://www.nelh.nhs.uk/screening/

Van Bogaert, L. J. (2006). Rights of and duties to non-consenting patients: informed refusal in the developing world. Developing World Bioethics, 6(1):13-22.

Van den Berg, M., Timmermanns, D. R. M., Kleinveld, J. H., Garcia, E., van Vugt, J. M. G. \& van der Wal, G. (2005). Accepting or declining the offer of prenatal screening for congenital defects: test uptake and women's reasons. Prenatal Diagnosis, 25(1):84-90.

Villeneuve, C., Laroche, C., Lippman, A., \& Marrache, M. (1988). Psychological aspects of ultrasound imaging during pregnancy. Canadian Journal of Psychiatry, 33(6):530-536.

Vintzeleos, A. M., Ananth, C. V., Smulian, J. C., Day-Salvatore, D. L, Beazoglou, T. \& Knuppel, R. A. (2000). Cost-benefit analysis of prenatal diagnosis for Down syndrome using the British or American approach. Obstetrics \& Gynecology, 95:577-583.

Voelker, R. (2005). The business of baby pictures: controversy brews over "keepsake" fetal ultrasounds. Journal of the American Medical Association, 293(1):25.

Wagner, M. (1994). Pursuing the Birth Machine: The Search for Appropriate Birth Technology. Sydney: ACE Graphics.

Wagner, M. (2001). Fish can't see water: the need to humanise birth. International Journal of Gynecology \& Obstetrics, 75:S25-S37.

Wald, N. J. \& Kennard, A. (1998). Routine ultrasound scanning for congenital abnormalities. Annals of the New York Academy of Sciences, 847:173-180.

Wald, N. J., Rodeck, C., Hackshaw, A. K. et al (2003). First and second trimester antenatal screening for Down's syndrome: the results of the Serum, Urine and Ultrasound Screening Study (SURUSS). Journal of Medical Screening, 10:56104.

Wapner, R., Thom, E., Simpson, J.L. et al. 2003. First-trimester maternal serum biochemistry and fetal nuchal translucency screening (BUN) study group. First trimester screening for trisomies 21 and 18. New England Journal of Medicine, 249:1405. 
Warren, S. \& Brewis, J. (2004). Matter over Mind? Examining the experience of pregnancy. Sociology, 38(2):219-236.

Wax, J. R. \& Pinette, M. G. (2006). Nonmedical fetal ultrasound-why all the noise? Birth, 33(1):1-3.

Weir, L. (1996). Recent developments in the government of pregnancy. Economy \& Society, 25(3):373-392.

Weir, L. (1998a). Pregnancy ultrasound in maternal discourse. In M. Shildrick \& J. Price (eds.), Vital signs: Feminist Reconfigurations of the Bio/logical Body. Edinburgh: Edinburgh University Press, pp 78-101.

Weir, L. (1998b). Cultural intertexts and scientific rationality: the case of pregnancy ultrasound. Economy \& Society 27(2):249-258.

Wertz, D. C. \& Fletcher, J. C. (1993). A critique of some feminist challenges to prenatal diagnosis. Journal of Women's Health, 2:173-188.

Wigglesworth, J. S. \& Singer, D. B. (eds.). Textbook of Fetal and Perinatal Pathology (2nd edition). Malden Massachusetts: Blackwell Science.

Williams, C., Alderson, P. \& Farsides, B. (2002a). Is non-directiveness possible within the context of antenatal screening and testing? Social Science \& Medicine, 54(3):339-347.

Williams, C., Alderson, P. \& Farsides, B. (2002b). Too many choices? Hospital and community staff reflect on the future of prenatal screening. Social Science \& Medicine, 55(5):743-753.

Williams, C., Alderson, P. \& Farsides, B. (2002c). Dilemmas encountered by health practitioners offering nuchal translucency screening: a qualitative case study. Prenatal Diagnosis, 22(3):216-220.

Williams, C., Sandall, J., Lewando-Hundt, G., Heyman, B., Spencer, K. \& Grellier, R. (2005). Women as moral pioneers? Experiences of first trimester antenatal screening. Social Science \& Medicine 61:1983-1992.

Williams, C. (2006). Dilemmas in fetal medicine: premature application of technology or responding to women's choice? Sociology of Health \& Illness, 28(1):1-20.

Wolbring, G. (2003). Disability rights approach towards bioethics? Journal of disability policy studies, 14(3):174-180.

World Health Organisation (WHO), (1985). Appropriate technology for birth. The Lancet, 326(8452):436-437.

World Health Organisation (WHO), (1996). Care in Normal Birth: A Practical Guide. 
Maternal Health and Safe Motherhood Programme, Division of Family Health. Geneva: WHO.

World Health Organisation (WHO), (2008). Primary Health Care (Now More Than Ever): The World Health Report 2008. Geneva: WHO.

Young, C., McMahon, J. E., Bowman, V. \& Thompson, D. (1989). Maternal reasons for delayed prenatal care. Nursing Research, 38(4):242-3.

Zadoroznyj, M. (2000). Midwife-led maternity services and consumer 'choice' in an Australian metropolitan region. Midwifery, 16:177-185.

Zechmeister, I. (2001). Foetal images: the power of visual technology in antenatal care and the implications for women's reproductive freedom. Health Care Analysis, 9(4):387-400. 